\title{
The Role of the Ras Guanyl-Nucleotide Exchange Factor Rasgrp1 in Synaptic Transmission
}

\author{
Dissertation \\ in partial fulfillment of the requirements for the degree "Doktor \\ rerum naturalium" in the GAUSS Program at the Georg- \\ August-University-Göttingen, Faculty of Biology.
}

Submitted by

Simon Rolf Bungers

Born in Bonn, Germany

June 2010 


\section{Declaration}

I hereby declare that this dissertation "The Role of the Ras Guanyl-Nucleotide

Exchange Factor Rasgrp1 in Synaptic Transmission" has been written independently, with no other aids than those cited.

I also declare that I am not enrolled or have applied for another university in order to graduate with the degree "Doktor rerum naturalium".

Göttingen, $7^{\text {th }}$ of June, 2010

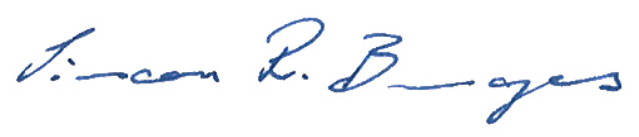

Simon Rolf Bungers

This dissertation was written at the Max-Planck-Institute of Experimental Medicine in the Department of Molecular Neurobiology.

$1^{\text {st }}$ Corrector: Prof. Dr. Nils Brose

$2^{\text {nd }}$ Corrector: Prof. Dr. Ralf Heinrich

Supervisor: Dr. Andrea Betz/ Prof. Dr. Nils Brose

Date of the Disputatio: June $24^{\text {th }}, 2010$ 
Index of Figures ............................................................................... 4

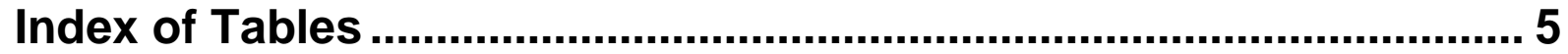

Abbreviations (........................................................................................ 6

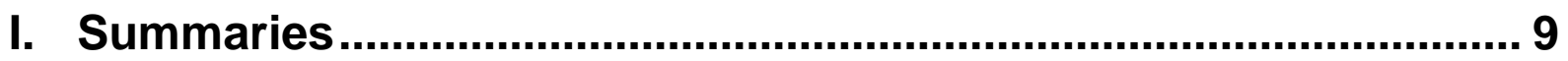

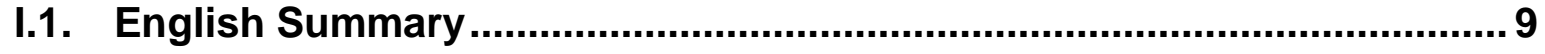

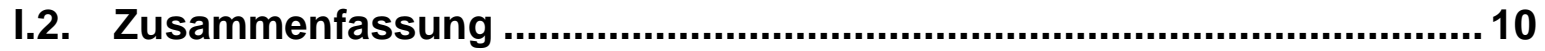

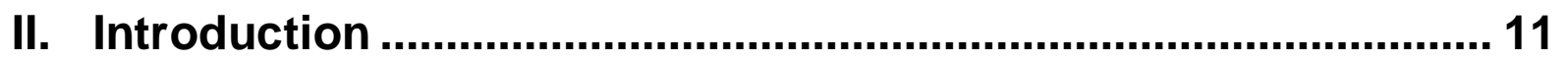

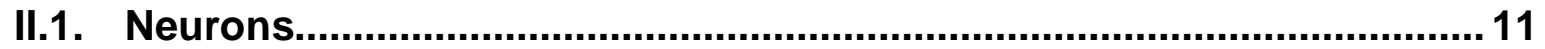

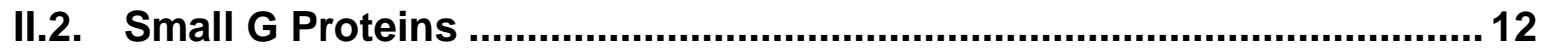

II.2.1. Complexity of the Ras Family of Small G Proteins............................ 13

II.2.2. The Classical Ras Signaling Cascade ........................................... 13

II.3. Controversies in the Research of Neuronal Ras Signaling ...................14

II.3.1. Ras Signaling and Morphology ................................................... 15

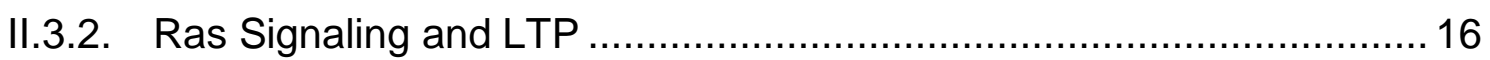

II.3.3. Ras Signaling and AMPAR Trafficking.......................................... 18

II.3.4. Postsynaptic and Presynaptic Effects of Ras Signaling ..................... 19

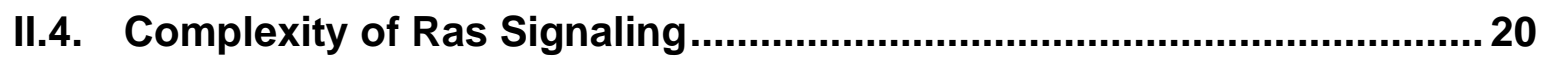

II.4.1. Multitude of Ras Downstream Pathways.......................................... 21

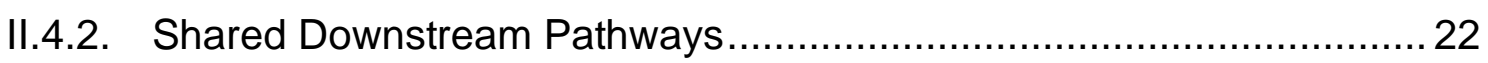

II.4.3. Pathway Selection by Ras G Proteins …......................................... 23



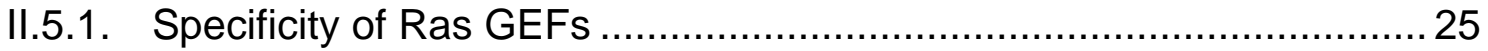

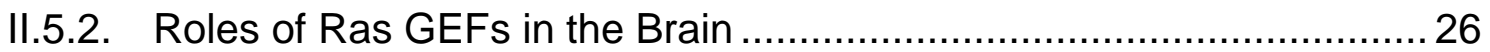

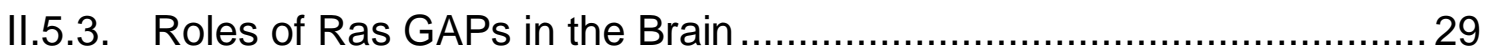

II.5.4. Interplay of Ras Regulatory Proteins in the Hippocampus ................... 31

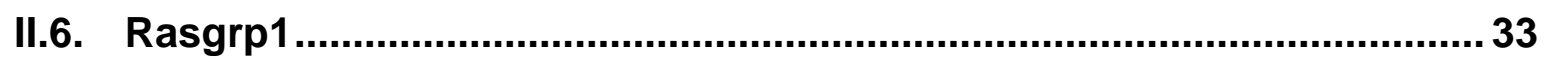

II.6.1. Rasgrp1 in Non-neuronal Functions ............................................. 33

II.6.2. Insights in the Function of Rasgrp1 in the Brain................................ 33

II.6.3. Structure of Rasgrp1 and Mechanisms of Function ............................ 34

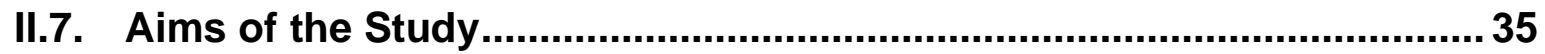

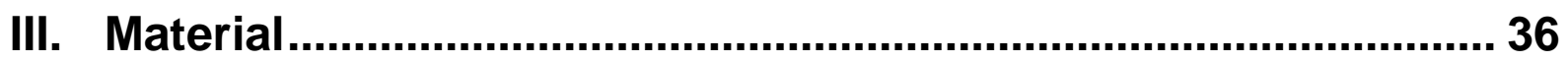




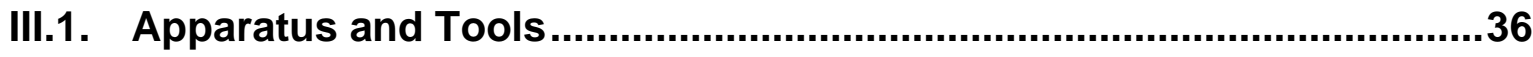

III.2. Consumables and Kits......................................................................36

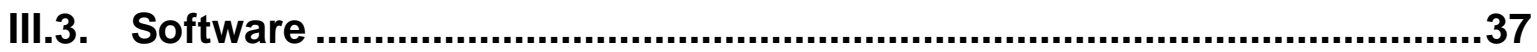

III.4. Chemicals, Media and Solutions............................................................

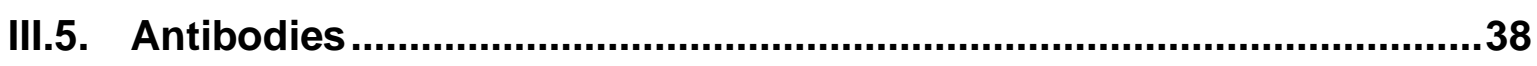

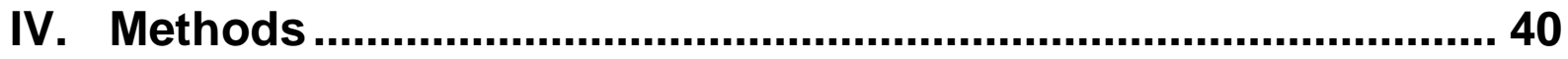

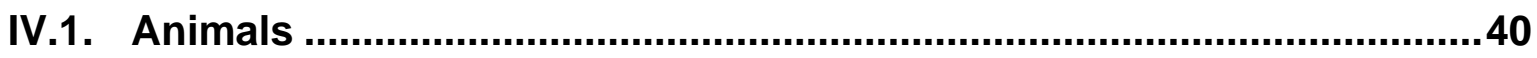

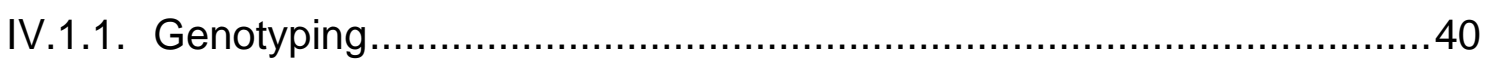

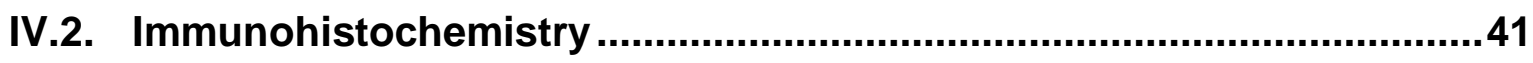

IV.3. Cell Culture ....................................................................................42

IV.3.1. Preparation of Primary Hippocampal Cell Cultures from P0 Mice........ 42

IV.3.2. Immunocytochemical Stainings..................................................... 43

IV.4. Microscopy and Image Analysis ...........................................................44

IV.4.1. Analysis of Dendrite Morphology Using Sholl Analysis (Sholl, 1953) ... 44

IV.4.2. Analysis of Synaptic Composition of the CA1 Region ..........................45

IV.4.2.1. Development and Evaluation of Image Processing Methods.......46

IV.4.2.2. The Processing Method of Choice............................................. 51

IV.5. Electrophysiology on Acute Hippocampal Slices .................................53

IV.5.1. Preparation of Acute Hippocampal Slices ……................................53

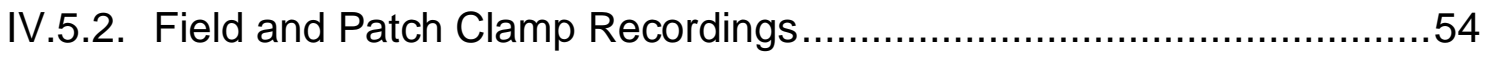

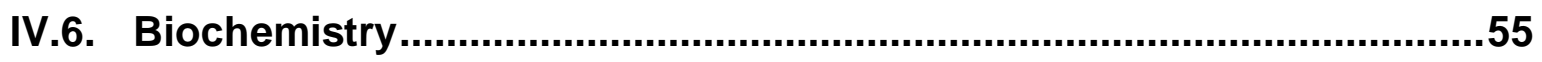

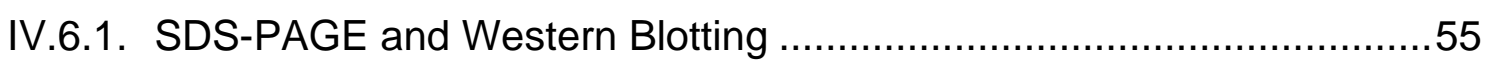

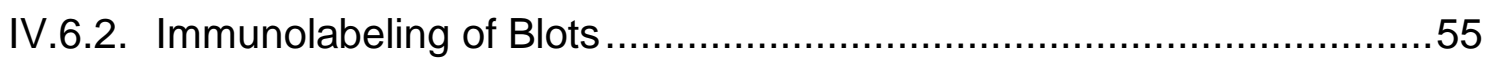

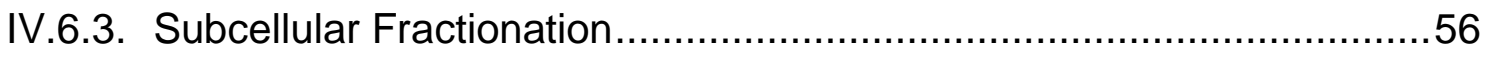

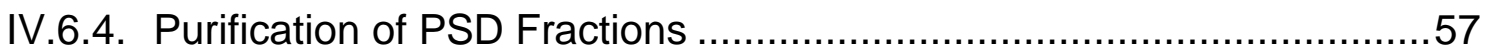



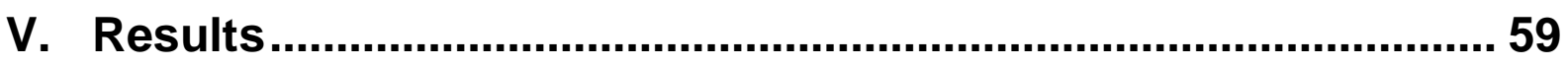

V.1. Distribution of Rasgrp1 in the Mouse Hippocampus...........................59

V.1.1. Strongest Expression of Rasgrp1 is Found in the

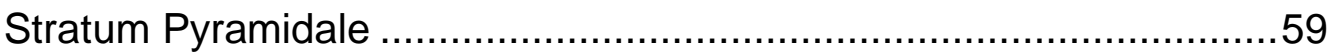

V.1.2. Rasgrp1 is Not Expressed in Inhibitory Neurons................................60

V.1.3. Rasgrp1 Preferentially Localizes to the Golgi Apparatus of Neurons...63

V.1.4. Enrichment of Rasgrp1 in the Microsomal Fraction of Fore Brain........65

V.2. Characterization of the Rasgrp1 KO Mouse .......................................67 
V.2.1. No Obvious Behavioral Phenotypic Changes or Defects in Rasgrp1 KO Mice 67

V.2.2. Gross Brain Morphology of Rasgrp1 KO Mice is Normal. 67

V.2.3. Rasgrp1 KO Neurons Display Aberrant Dendrite Morphogenesis ....... 71

V.2.4. Rasgrp1 KO Mice Show an Increased Synaptic Transmission............ 75

V.2.4.1. Field Recordings in the Rasgrp1 KO Hippocampus .................... 75

V.2.4.2. Patch Clamp Recordings in the Rasgrp1 KO Hippocampus ........8 80

V.2.5. Rasgrp1 KO Mice Show Increased PSD-95 Expression ..................... 84

V.2.5.1. Imaging of Synaptic Composition in the Rasgrp1 KO Mouse....... 84

V.2.5.2. Biochemical Analysis of Postsynaptic Markers in the Rasgrp1 KO Hippocampus

VI. Discussion. 103

VI.1. Lack of Rasgrp1 Increases Postsynaptic Sensitivity. 103

VI.2. An Increase of PSD-95 Levels in the Hippocampus of Rasgrp1 KO Mice Mediates Increased Synaptic Efficiency 104

VI.3. Ras Signaling and Synaptic Transmission 107

VI.4. Localized Regulation of Neuronal Ras Function................................108

VI.5. A Possible Role for Rasgrp1 in Synaptogenesis ................................109

VI.6. A Possible Role of Rasgrp1 in Dendrite Morphogenesis ...................111



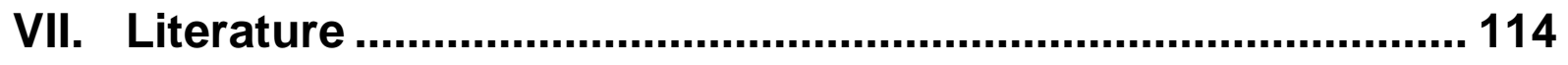

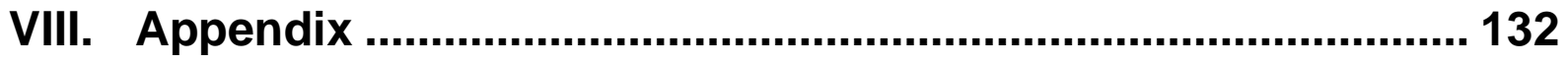

VIII.1. Strategy for the Knock-out of Rasgrp1 in Mice ..................................132

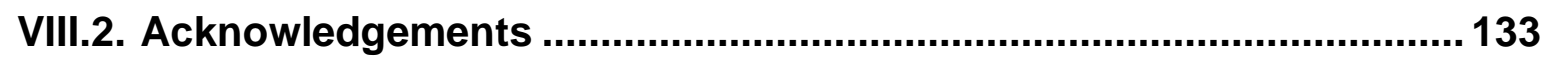

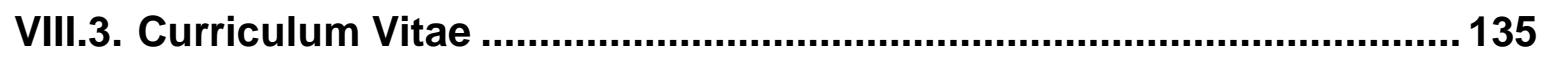




\section{Index of Figures}

Figure IV.1: Macro frameset for the analysis of synaptic composition.

Figure IV.2: Evaluation of the image processing method for analysis of synaptic composition .49

Figure IV.3: Visual control of processing and particle selection .50

Figure V.1: Regional distribution of Rasgrp1 in the CA1 region of the hippocampus 60

Figure V.2: Cellular distribution of Rasgrp1 in the stratum pyramidale... .62

Figure V.3: Subcellular localization of Rasgrp1 in pyramidal neurons. .64

Figure V.4: Fore brain subcellular fractions containing Rasgrp1 .........................66

Figure V.5: Observation of Rasgrp1 KO mice and brains..................................68

Figure V.6: Gross brain morphology of Rasgrp1 KO mice ................................69

Figure V.7: Analysis of dendrite morphogenesis in Rasgrp1 KO neurons................73

Figure V.8: Field recordings in the CA1 stratum radiatum of Rasgrp1 $\mathrm{KO}$ mice........78

Figure V.9: Patch clamp recordings in the CA1 stratum pyramidale of Rasgrp1 KO mice .82

Figure V.10: Imaging of excitatory postsynapses in CA1 of Rasgrp1 KO mice .87

Figure V.11: Imaging of presynapses in the CA1 region of Rasgrp1 KO mice .91

Figure V.12: Imaging of excitatory presynapses in the CA1 region of

Rasgrp1 KO mice .94

Figure V.13: Imaging of inhibitory presynapses in the CA1 region of

Rasgrp1 KO mice

Figure V.14: Biochemical analysis of hippocampal homogenates and PSD fractions prepared from Rasgrp1 KO mice.

Figure VIII.1: Organization of the RasGRP1 gene and construction of the targeting vector in pFlexHR (Schnutgen et al., 2003). 132 


\section{Index of Tables}

Table V.1: Input-output experiments in Rasgrp1 KO hippocampal slices 75

Table V.2: Paired pulse facilitation in Rasgrp1 KO hippocampal slices .76

Table V.3: $14 \mathrm{~Hz}$ experiments in Rasgrp1 KO hippocampal slices ........................ 77

Table V.4: Intensities of PSD-95 puncta in the Rasgrp1 KO hippocampus................ 85

Table V.5: Numbers and areas of PSD-95 puncta in the Rasgrp1 KO

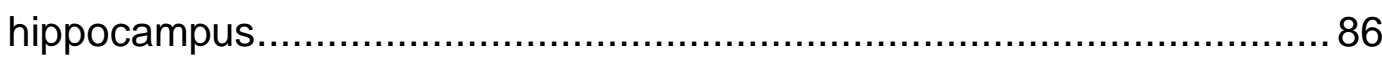

Table V.6: Intensities of Synapsin1/2 puncta in the Rasgrp1 KO hippocampus........ 90

Table V.7: Numbers and areas of Synapsin1/2 puncta in the Rasgrp1 KO

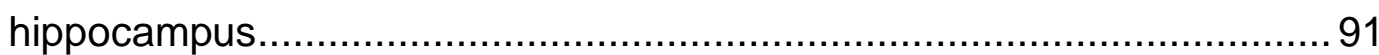

Table V.8: Intensities of VGLUT1 puncta in the Rasgrp1 KO hippocampus .............93

Table V.9: Numbers and areas of VGLUT1 puncta in the Rasgrp1 KO

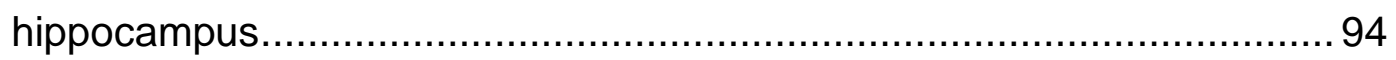

Table V.10: Intensities of VGAT puncta in the Rasgrp1 KO hippocampus ............... 96

Table V.11: Numbers and areas of VGAT puncta in the Rasgrp1 KO hippocampus 96

Table V.12: Relative protein content of markers in homogenate and

PSD fractions 


\section{Abbreviations}

\section{Protein abbreviations}

\begin{tabular}{|c|c|c|}
\hline $\begin{array}{l}\text { Official } \\
\text { Symbol }\end{array}$ & Name & $\begin{array}{l}\text { Other designations, } \\
\text { notes }\end{array}$ \\
\hline Calb1 & Calbindin & D-28K, calbindin D28 \\
\hline Calb2 & Calretinin, Calbindin2 & $\mathrm{CR}$ \\
\hline Camk2a & calcium/calmodulin-dependent protein kinase II alpha & Camkll subunit alpha \\
\hline Canx & Calnexin & Cnx \\
\hline Creb1 & cAMP responsive element binding protein 1 & \\
\hline Dab2ip & disabled homolog 2 (Drosophila) interacting protein & \\
\hline Dgkz & diacylglycerol kinase zeta & \\
\hline Diap1 & diaphanous homolog 1 & Dia1 \\
\hline Dlg2 & disks large homolog 2 & $\begin{array}{l}\text { PSD-93, Psd93, Chapsyn- } \\
110\end{array}$ \\
\hline Dlg4 & disks large homolog 4 & PSD-95, Psd95 \\
\hline Eea1 & early endosome antigen 1 & \\
\hline Egf & Epidermal growth factor & \\
\hline Gad1 & glutamic acid decarboxylase 1 & $\begin{array}{l}\text { EP10, GAD25, GAD44, } \\
\text { GAD67 }\end{array}$ \\
\hline Gad2 & glutamic acid decarboxylase 2 & GAD65 \\
\hline Golgb1 & Giantin, golgi autoantigen, golgin subfamily b, macrogolgin 1 & Gm6840 \\
\hline Grb2 & growth factor receptor-bound protein 2 & \\
\hline Gria1 & glutamate receptor, ionotropic, AMPA1 (alpha 1) & GluR1, GluA1, GluRA \\
\hline Gria2 & glutamate receptor, ionotropic, AMPA2 (alpha 2) & $\begin{array}{l}\text { GluR2, GluA2, GluR-B, Glur- } \\
2\end{array}$ \\
\hline Gria3 & glutamate receptor, ionotrophic, AMPA 3 & $\begin{array}{l}\text { GluR3, GLUR3, GluA3, } \\
\text { GluR-3 }\end{array}$ \\
\hline Grin1 & glutamate receptor, ionotropic, NMDA1 (zeta 1) & NR1, Nmdar, NMDAR1, \\
\hline Grin2a & glutamate receptor, ionotropic, NMDA2A (epsilon 1) & NR2A, NMDAR2A \\
\hline Grin2b & glutamate receptor, ionotropic, NMDA2B (epsilon 2) & NR2B, NMDAR2B \\
\hline Gripap1 & GRIP1 associated protein 1 & GRASP-1 \\
\hline Hras1 & Harvey rat sarcoma virus oncogene 1 & H-Ras, Ha-ras, Hras-1, Hras \\
\hline lqgap & IQ motif containing GTPase activating protein & several isoforms \\
\hline Kndc1 1 & kinase non-catalytic C-lobe domain (KIND) containing 1 & VKIND \\
\hline Kras & v-Ki-ras2 Kirsten rat sarcoma viral oncogene homolog & Kras2, Ki-ras, Kras-2 \\
\hline Ksr1 & kinase suppressor of ras 1 & \\
\hline Map2 & microtubule associated protein 2 & \\
\hline Map2 & microtubule-associated protein 2 & MAP2R, Mtap2 \\
\hline Map2k & Mitogen activated protein kinase kinases & several isoforms \\
\hline Map2k1 & Mitogen activated protein kinase kinase 1 & Mek1 \\
\hline Map2k2 & Mitogen activated protein kinase kinase 2 & Mek2 \\
\hline Mapk & $\begin{array}{l}\text { Mitogen activated protein kinases, initially also known as Map2 } \\
\text { kinase }\end{array}$ & Erk, several isoforms \\
\hline Mapk1 & Mitogen activated protein kinase 1 & Erk2, p42 Mapk \\
\hline Mapk14 & Mitogen activated protein kinase 14 & $\mathrm{p} 38 \mathrm{a}$ \\
\hline Mapk2 & Mitogen activated protein kinase 2 & Erk1, p44 Mapk \\
\hline MIl1 & myeloid/lymphoid or mixed-lineage leukemia & ALL-1 \\
\hline Mllt4 & myeloid/lymphoid or mixed-lineage leukemia 4 & Afadin \\
\hline Mras & muscle and microspikes RAS & M-Ras, R-Ras3 \\
\hline Nf1 & Neurofibromin 1 & \\
\hline Nras & neuroblastoma ras oncogene & N-Ras \\
\hline Pi3k & Phosphoinositide- 3 kinase & \\
\hline Pi3kc & phosphatidylinositol 3-kinase catalytic & p110, several isoforms \\
\hline Pi3kr & regulatory subunit of Pi3k & p85, several isoforms \\
\hline Plce & phospholipase C- $\varepsilon$ & several isoforms \\
\hline Pld & Phospholipase D & several isoforms \\
\hline Pvalb & Parvalbumin & PV, Pva, Parv, Pvalb \\
\hline Ralbp1 & Ral binding protein 1 & \\
\hline Ralqds & Ral guanine nucleotide dissociation stimulator & \\
\hline Rap1a & Ras related protein 1a & Rap1 \\
\hline Rapgef1 & Rap guanine nucleotide exchange factor (GEF) 1 & C3G, Grf2 \\
\hline Rapgef2 & Rap guanine nucleotide exchange factor (GEF) 2 & $\begin{array}{l}\text { CNrasGEF, nRap-GEF, PDZ- } \\
\text { GEF }\end{array}$ \\
\hline Rapgef3 & Rap guanine nucleotide exchange factor (GEF) 3 & Epac1 \\
\hline Rasa1 & RAS p21 protein activator 1 & $\begin{array}{l}\text { p120GAP, Rasa, RasGAP, } \\
\text { GAP1 }\end{array}$ \\
\hline Rasa2 & RAS p21 protein activator 2 & mGAP1, GAP1m \\
\hline Rasa3 & RAS p21 protein activator 3 & $\begin{array}{l}\text { GapllI, GAP1(IP4BP), R-Ras } \\
\text { GAP }\end{array}$ \\
\hline Rasa4 & RAS p21 protein activator 4 & Capri \\
\hline Rasal1 & RAS protein activator like 1 (GAP1 like) & MRASAL \\
\hline Rasal2 & RAS protein activator like 2 & NGAP \\
\hline
\end{tabular}




\section{Protein abbreviations (continued)}

\begin{tabular}{|c|c|c|}
\hline $\begin{array}{l}\text { Official } \\
\text { Symbol }\end{array}$ & Name & $\begin{array}{l}\text { Other designations, } \\
\text { notes }\end{array}$ \\
\hline Rasgrf1 & Ras guanyl releasing factor 1 & $\begin{array}{l}\text { Cdc25, Grf1, GNRB, P140 } \\
\text { RAS-GEF, P190. Ras-GRF1 }\end{array}$ \\
\hline Rasgrf2 & Ras guanyl releasing factor 2 & GRF2, Ras-GRF2 \\
\hline Rasgrp1 & Ras guanyl releasing protein 1 & Caldag-GEFII \\
\hline${\text { Rasgrp} 2 a^{1}}$ & Ras guanyl releasing protein 2 , isoform a & $\begin{array}{l}\text { Caldag-GEFI, shorter } \\
\text { Rasgrp2 isoform }\end{array}$ \\
\hline Rasgrp2 $b^{1}$ & Ras guanyl releasing protein 2 , isoform b & $\begin{array}{l}\text { Rasgrp2, longer Rasgrp2 } \\
\text { isoform }\end{array}$ \\
\hline Rasgrp3 & Ras guanyl releasing protein 3 & Caldag-GEFIII \\
\hline Rasgrp4 & Ras guanyl releasing protein 4 & Caldag-GEFIV \\
\hline Rassf & Ras association domain-containing & several isoforms \\
\hline Rin1 & Ras interaction/interference protein-1 & \\
\hline Rras & Harvey rat sarcoma oncogene, subgroup $\mathrm{R}$ & R-Ras \\
\hline Rras2 & related RAS viral (r-ras) oncogene homolog 2 & TC21 \\
\hline Slc17a7 & $\begin{array}{l}\text { solute carrier family } 17 \text { (sodium-dependent inorganic phosphate } \\
\text { cotransporter), member } 7\end{array}$ & \\
\hline Sos & son of sevenless homolog (Drosophila) & two isoforms \\
\hline Syngap1 & synaptic Ras GTPase activating protein 1 homolog & \\
\hline Syp & Synaptophysin & several isoforms \\
\hline Tiam1 & T-cell lymphoma invasion and metastasis- 1 & \\
\hline Wasl & Wiskott-Aldrich syndrome-like & N-WASP \\
\hline Wnt2 & wingless-related MMTV integration site 2 & Mirp, Irp \\
\hline
\end{tabular}

Bold words indicate the names/symbols used throughout the text; 1 = designation used in this study.

\section{General abbreviations}

\begin{tabular}{|c|c|}
\hline Abbreviation & Full designation \\
\hline$\sim$ & approximately \\
\hline$\sigma$ & standard deviation \\
\hline$\mu$ & mean \\
\hline$\mu \mathrm{L}$ & micro-liter \\
\hline$A$ & area \\
\hline aa & amino acid \\
\hline ACSF & artificial cerebro-spinal fluid \\
\hline AMPAR & [alpha]-amino-3-hydroxyl-5-methyl-4-isoxazolepopionic acid sensitive receptor \\
\hline AZ & active zone \\
\hline $\mathrm{bp}$ & basepairs \\
\hline $\mathrm{CA}$ & "Cornu ammonis" (horn of ammon) \\
\hline $\mathrm{cKO}$ & conditional $\mathrm{KO}$ \\
\hline $\mathrm{cKO}$ & conditional knock-out \\
\hline$C_{V}$ & coefficient of variation \\
\hline DAG & diacylglycerol \\
\hline DG & dentate gyrus \\
\hline DIV & day in-vitro \\
\hline DKO & double $\mathrm{KO}$ \\
\hline DMEM & Dulbecco's modified eagle medium \\
\hline DNA & desoxyribonucleotide acid \\
\hline $\mathrm{E}$ & embryonal stage \\
\hline EM & electron microscopy / electron microscope \\
\hline ER & endoplasmatic reticulum \\
\hline FBS & fetal bovine serum \\
\hline fEPSP & field excitatory postsynaptic potential \\
\hline FRET & fluorescent resonance energy transfer \\
\hline G12V & Glycine to Valine mutation at aaposition 12 \\
\hline GABA & $\gamma$-Aminobutyric acid (4-aminobutanoic acid) \\
\hline GAP & GTPase activating protein \\
\hline GDP & guanosine-diphosphate \\
\hline GEF & guanine exchange factor \\
\hline GS & goat serum \\
\hline GTP & guanosine-5'-triphosphate \\
\hline i.e. & it est ("that is") \\
\hline $\mathrm{IP}_{3}$ & inositol-trisphosphate \\
\hline $\mathrm{kb}$ & kilo-basepairs \\
\hline $\mathrm{KO}$ & knock out, synonym for the functional disruption of a gene in an organism \\
\hline L-DOPA & L-3,4-dihydroxyphenylalanine ((S)-2-amino-3-(3,4-dihydroxyphenyl) propanoic acid) \\
\hline LTD & long-term depression \\
\hline LTP & long-term potentiation \\
\hline mEPSC & mini excitatory postsynaptic currents \\
\hline
\end{tabular}


General abbreviations (continued)

\begin{tabular}{|c|c|}
\hline Abbreviation & Full designation \\
\hline mRNA & messenger ribonucleic acid \\
\hline NCC & sodium chloride transporter \\
\hline NMDAR & N-methyl-D-aspartic acid sensitive receptor \\
\hline nr. & number \\
\hline NT & neurotransmitter \\
\hline $\mathrm{P}$ & postnatal stage \\
\hline$P$ & perimeter \\
\hline PC12 & pheochromocytoma cell line $\mathrm{nr} .12$ \\
\hline PCR & polymerase chain reaction \\
\hline PFA & paraformaldeyde \\
\hline $\mathrm{PIP}_{2}$ & phosphatidyl inositol-bisphosphate \\
\hline PPF & paired-pulse facilitation \\
\hline PSD & postsynaptic density \\
\hline PT & plasma-targeting domain \\
\hline$r$ & restriction value \\
\hline Ras & rat sarcoma \\
\hline Ras-GTP & GTP bound Ras G protein \\
\hline $\mathrm{ROI}$ & region of interest \\
\hline SER & smooth endoplasmatic reticulum \\
\hline SLE & Systemic lupus erythematosis \\
\hline slm & stratum lacunosum moleculare \\
\hline small G protein & small GTP/GDP binding protein \\
\hline so & stratum oriens \\
\hline $\mathrm{sp}$ & stratum pyramidale \\
\hline $\mathrm{sr}$ & stratum radiatum \\
\hline SuPT & suppressor of plasma targeting domain \\
\hline TCR & T-cell receptor \\
\hline TTX & Tetrodotoxin \\
\hline WT & wild-type \\
\hline
\end{tabular}




\section{Summaries}

\section{I.1. English Summary}

Ras signaling pathways are known to regulate neuronal development and function. Ras $G$ proteins have been implicated in the differentiation of neurons, e.g. in dendrite and dendritic spine morphogenesis, and in processes such as the regulation of synaptic transmission and plasticity. The effects of neuronal signaling by Ras $G$ proteins appear to be highly pleiotropic, and in the past, the complexity of Ras signaling has led to substantial controversies in the field of Ras signaling research. To contribute to a better understanding of Ras signaling in neurons, I investigated the role of Rasgrp1, a protein that regulates signaling by Ras $G$ proteins.

Rasgrp1 is a Ras guanyl-nucleotide exchange factor (GEF) that is highly expressed in the hippocampus. Although the neuronal roles of other Ras GEFs and also of several Ras GTPase activating proteins (GAPs) have been investigated in the past, the neuronal function of Rasgrp1 has not been studied so far. The results presented in this study show that Rasgrp1 deficient mice exhibit an increase in basal synaptic transmission. This increase in transmission is caused by an enhanced postsynaptic sensitivity, which, in turn, most likely results from an increase in PSD-95 expression at the postsynapse and a consequent increase in glutamate receptor function. The effects of Ras signaling appear to be not only regulated by mere activation and inactivation but also by the location, at which the regulation of Ras $G$ proteins takes place. The present study shows that Rasgrp1 is exclusively expressed in pyramidal neurons, where it primarily localizes to the Golgi apparatus.

Taken together, this is the first study to provide evidence for a specific neuronal function of Rasgrp1. It shows that Rasgrp1 rather selectively controls postsynaptic sensitivity at glutamatergic synapses. In terms of Ras signaling in general, the present study shows that selective perturbation of individual Ras control pathways is a very useful tool to dissect the pleiotropic effects of Ras function in neurons. 


\section{I.2. Zusammenfassung}

Seit Längerem ist bekannt, dass die Signaltransduktion durch Ras Proteine an der neuronalen Entwicklung und Funktion beteiligt ist. Ras Proteine spielen sowohl eine wichtige Rolle in der Differenzierung von Neuronen, z.B. in der Morphogenese von Dendriten und dendritischen Dornen, als auch in der Regulation der synaptischen Transmission und Plastizität. Die direkte Aktivierung von Ras Proteinen hat eine Vielzahl von möglichen Effekten zur Folge, was die experimentelle Erforschung von spezifischen Funktionen der Ras Signalwege sehr erschwert und in der Vergangenheit immer wieder zu kontroversen Ergebnissen geführt hat. Die vorliegende Studie untersucht das Protein Rasgrp1, das die Aktivierung von Ras Proteinen reguliert.

Rasgrp1 ist ein Ras Guanyl-Nucleotid Austausch Faktor (englisch: guanlynucleotide exchange factor, GEF) und wird in hohem Maße im Hippocampus exprimiert. Viele andere GEFs und auch GTPase aktivierende Proteine (GAPs), die Ras Proteine regulieren, wurden schon auf ihre neuronale Funktion hin untersucht. Im Gegensatz dazu wurde die Funktion von Rasgrp1 bis jetzt noch nicht erforscht. Die Ergebnisse meiner Studie zeigen, dass genetische Deaktivierung von Rasgrp1 in Mäusen zu erhöhter synaptischer Transmission führt. Diese Erhöhung lässt sich auf eine verstärkte postsynaptische Effizienz zurückführen, die höchstwahrscheinlich von einer verstärkten Exprimierung von PSD-95 an der Postsynapse und einer damit verbundenen, erhöhten Funktion von Glutatmat-Rezeptoren herrührt. Die Effekte von Ras Signaltransduktionswegen werden nicht nur durch die bloße Aktivierung oder Inaktivierung von Ras Proteinen reguliert, sondern in einem zunehmend beachteten Maße auch durch den Ort in der Zelle, an dem diese Regulation auftritt. Meine Ergebnisse zeigen, dass Rasgrp1 ausschließlich in den Pyramidenzellen des Hippocampus exprimiert wird. In den Pyramidenzellen wird Rasgrp1 hauptsächlich am Golgi- Apparat detektiert.

Diese Studie enthält die ersten Beweise für eine spezifische neuronale Funktion von Rasgrp1. Sie zeigt, dass Rasgrp1 selektiv die postsynaptische Sensitivität an glutamatergen Synapsen reguliert. Diese Studie zeigt, dass die selektive Veränderung der Regulation von Ras eine hilfreiche Methode ist, um die vielfältigen Effekte der Ras Signaltransduktion in Neuronen verstehen zu können. 


\section{Introduction}

\section{II.1. Neurons}

The nervous system has to achieve three tasks to function: First, to set itself up during development, second, to reliably integrate input signals into information that can be stored and third, to generate output signals.

In early development, neuronal progenitors arise from epithelial cells, proliferate and subsequently differentiate into many types of nerve cells. These neurons are the smallest autonomous units of the nervous system. Neurons typically consist of a soma and thin processes called neurites, which extend far into the surrounding area. In early neuronal development, these neurites grow out of the soma. They differentiate into the relatively thick dendrites that receive signals, and a thinner neurite that sends signals and that is called axon. The sent signal is actively conducted through the axon and is called action potential. Depolarization of an axonal segment leads to opening of voltage-gated ion channels at adjacent sites, resulting in depolarization of the next segment. To transmit signals from one to another cell, neurons form synapses in later stages of development. The sending side of the synapse is called presynapse and is represented by a small bouton formed at the tip of the axon. When the action potential reaches the presynaptic bouton, voltage gated calcium channels open and increasing calcium concentration within the bouton triggers vesicle fusion and neurotransmitter release. The most abundant neurotransmitter in the brain is glutamate. glutamate passively diffuses from the presynaptic sites to the receiving cell. The highest concentration of glutamate receptors is found at small, but highly specialized compartments, called spines. These small protrusions that decorate the dendrites of the receiving cell represent the postsynapse. Upon binding of Glutamate, the receptors open and allow Sodium to enter the cell. Upon this influx of cations, the cell depolarizes locally. This electrical signal propagates as the depolarization passively spreads through the dendrites. Spine shape, dendrite caliber and branching influence signal propagation as it travels to the soma. In the soma, final integration of all incoming signals takes place. If the resulting depolarization passes a certain threshold, a new action potential is generated in an all-or-none fashion.

Postsynaptic spines, dendrites, the soma, the axon and presynaptic boutons represent highly specialized compartments that are part of the complex morphology of neurons. In fact, without knowledge of ion channels or biophysical properties of the 
membrane, careful observation of this complex morphology has led to the first ideas on how a single nerve cells could transmit signals and thereby contribute to the function of the nervous system (Golgi, 1873; Purkyně, 1838; Ramón y Cajal, 1888). Later, in times of modern neuroscience, changes in morphology such as in postsynaptic spine shape could be correlated to information processing and storage. By now, many molecular cues involved in the development, morphogenesis, signal transmission and information storage are known.

One superfamily of proteins involved in all of these processes is the superfamily of small G proteins.

\section{II.2. Small G Proteins}

Small G proteins (small GTP/GDP binding proteins, also designated G proteins in this text) can be found in a guanosine-5'-triphosphate (GTP) bound and in a guanosine-diphosphate (GDP) bound state. In the GTP bound state, they bind to effectors and thereby activate molecular signaling cascades. Their activity is terminated, when GTP is hydrolyzed to GDP by an intrinsic GTPase activity (Ehrhardt et al., 2002; Stryer et al., 2002). The superfamily of small G proteins comprises of more than 150 proteins (Bernards, 2006) that are structurally classified into five families. These five families are the Ras, Rho, Rab, Arf and Ran families.

In the classical view, Ras family $G$ proteins signal via the Mitogen-activatedprotein-kinase-kinase (Map2k1, also known as "Mapk/Erk kinase", Mek), the Phosphoinositide-3 kinase (Pi3k) or the Ral guanine nucleotide dissociation stimulator (Ralgds) pathways to function in proliferation, differentiation and apoptosis by regulating gene expression (Takai et al., 2001).

Rho family $G$ proteins regulate cytoskeletal rearrangements through actin binding proteins such as Wasl (Wiskott-Aldrich syndrome-like, also known as $\mathrm{N}$ WASP) and Diap1 (diaphanous homolog 1, also known as Dia1). In this way, they function in the formation of stress fibers, lamellipodia and other morphological processes. In addition, they also regulate gene expression and signal via Pi3k (Takai et al., 2001). Rab family $G$ proteins function in protein sorting, intracellular vesicle trafficking, targeting, docking and fusion. Their mechanism is to activate effectors that directly influence vesicular membrane shape, vesicle tethering or vesicle motility. (Stenmark, 2009; Takai et al., 2001). By similar mechanisms, Arf G proteins function in intracellular trafficking, in particular in vesicle budding from endomembranes, in 
endocytosis, but also in cytoskeletal reorganization (Takai et al., 2001). Ran family proteins make up the smallest family of small $G$ proteins and mediate nucleocytoplasmic transport (Takai et al., 2001).

Modern views (Goldfinger, 2008; Helmreich, 2004; Karnoub and Weinberg, 2008) of small $G$ protein signaling pay more attention to downstream processes are distinct from the canonical pathways, to spatial and temporal dynamics and finally, to cross talk between $G$ proteins within one or different families.

\section{II.2.1. Complexity of the Ras Family of Small G Proteins}

The Ras family of small $G$ proteins (not to be mistaken for the Ras superfamily) controls proliferation, cell migration, adhesion, apoptosis, differentiation and cell function (Goldfinger, 2008) and contains at least 36 members (Karnoub and Weinberg, 2008). Hras, the first small G protein discovered (DeFeo et al., 1981; Harvey, 1964), and its close relatives Nras and Kras form their own subfamily and share the same effector binding region (Goldfinger, 2008). $\mathrm{H}$-, N- and Kras are also known as the "classical Ras proteins" or the "p21 Ras proteins". The genes encoding these proteins were discovered in rats that developed sarcoma (a cancer of the connective or supportive tissue) upon retroviral infection. In these tumors, the ras genes (rat sarcoma) were found to be mutated. Later findings indicated, that the ras genes are mutated in many human tumors and subsequently led to intensive research on these genes (Karnoub and Weinberg, 2008).

In the literature the Ras family is further divided into the Mras/Rras, Ral, Rit, Rap and Rheb subfamilies. These subfamilies encompass only half of the Ras proteins found, the other members found in databases can be grouped into Rem, Rerg, Diras/Rasd and Nkiras families. It is important to note that the most intensely investigated Ras proteins belong to the Ras and Rap subfamilies. While data on neuronal functions of the Rras/Mras, Ral and Rheb subfamilies are slowly emerging, the vast majority of Ras $G$ proteins still await neurobiological attention.

\section{II.2.2. The Classical Ras Signaling Cascade}

The first findings indicating a neuronal involvement of the classical Ras $G$ proteins came from experiments using the pheochromocytoma cell line (PC12). Overexpression of Hras, Nras or infection with the Kirsten murine sarcoma virus led to neuronal differentiation of these cells, which was recognized by the outgrowth of 
neurites and cessation of cell division (Bar-Sagi and Feramisco, 1985; Guerrero et al., 1986; Noda et al., 1985). This function in neurite outgrowth was later verified on primary chick embryonic neurons, using overexpression of constitutively active Ras constructs (Borasio et al., 1989).

Classical Ras proteins moved into the neurobiological spotlight when they were found as the missing piece in a molecular signaling cascade that governs long-term potentiation (LTP). It had already been known that LTP requires influx of calcium ions into the cell and ultimately depends on activation of gene expression (Davis and Squire, 1984). The effectors most downstream in this cascade, mitogen-activated kinases (Mapk, also known as "Extracellular signal regulated kinases", Erk) were found to be phosphorylated and activated by NMDAR ( $N$-methyl-D-aspartic acid sensitive receptor) stimulation (Bading and Greenberg, 1991). In 1992, Ras was found to be involved in Mapk phosphorylation and activation, although it was clear that Ras itself could not phosphorylate Mapk (Leevers and Marshall, 1992; Wood et al., 1992). Next, proof was provided for Ras binding of the Raf kinase, which subsequently leads to phosphorylation and activation of Map2k (Vojtek et al., 1993; Warne et al., 1993; Zhang et al., 1993). One year later it was discovered that Map2k phosphorylates Mapk (Rosen et al., 1994), completing the Ras/Raf/Map2k/Mapk cascade. In neurons, a putative involvement of this cascade in a calcium sensitive pathway had been hypothesized earlier (Bading et al., 1993). Finally, evidence was provided for the phosphorylation and activation of Mapk1 upon electrophysiological LTP induction (English and Sweatt, 1996) and Ras proteins acquired a prominent position in the molecular machinery of LTP formation (Finkbeiner and Greenberg, 1996).

\section{II.3. Controversies in the Research of Neuronal Ras Signaling}

Since publication of the findings described above, research on neuronal Ras signaling has led to many controversies. In this regard, one experimental system in particular appears to have given rise to most of the controversies in the field. Extensive research on Ras signaling is conducted using activated mutants of Ras proteins. The activated mutated protein is able to bind GTP, but unable to hydrolyze it to GDP and therefore remains in a constitutively active state (Karnoub and Weinberg, 2008). The corresponding frequently used Glycine to Valine mutation at amino acid (aa) position $12(\mathrm{G} 12 \mathrm{~V})$ is normally found in oncogenic Hras. 
Endogenous $\mathrm{G} 12 \mathrm{~V}$ forms of $\mathrm{H}-, \mathrm{N}-$, and $\mathrm{Kras}$ can be found in almost every human tumor, implying that normally, Ras activity in cells is very tightly controlled.

Circumventing this control was thought to facilitate investigation of Ras proteins and their effects in neurons. In addition to the commonly used overexpression of exogenous G12V Hras in cultured cells and organotypic cultures, two independent research groups generated two very similar mouse models to investigate endogenously expressed G12V Hras. Both the "Synras mouse" (Arendt et al., 2004; Heumann et al., 2000; Seeger et al., 2004) and the "Ras ${ }^{\mathrm{G} 12 \mathrm{~V}}$ mouse" (Kushner et al., 2005) selectively express G12V Hras in postmitotic neurons, the time point of expression being controlled by the Synapsin1 or the Camk2a promoter, respectively. Strikingly, not only the phenotypes of these mice differ significantly in most aspects studied so far. Also the results of these gain-of-function studies cannot be correlated to the results from a loss-of-function study performed by knocking out Hras (Manabe et al., 2000). In the following, I will recollect these and other findings that question the validity of the model systems used and the conclusions concerning Ras signaling in neurons that were drawn based on them.

\section{II.3.1. Ras Signaling and Morphology}

The most controversial results of the characterization of the Synras and the Ras $^{\mathrm{G} 12 \mathrm{~V}}$ mice concern the findings on postsynaptic morphology. The Synras mice were found to have an increased cortical volume $(\sim 15 \%)$ that results from general neuronal hypertrophy (Heumann et al., 2000). In an extensive sequence of morphological studies, soma size, dendritic size and diameter, dendritic complexity and spine density were found to be significantly increased in this mouse model (Alpar et al., 2003; Arendt et al., 2004; Gärtner et al., 2005; Holzer et al., 2001; Seeger et al., 2003). Also axonal diameters (but not the numbers of axons) were found to be increased. However, no gross morphological changes were observed in Ras ${ }^{\mathrm{G} 12 \mathrm{~V}}$ mice, in particular any increase of dendritic spine density. Moreover, a detailed EM study did not reveal any abnormalities in postsynaptic morphology. The only consistent finding was a significantly increased number of docked synaptic vesicles at presynaptic active zones (AZ) (Kushner et al., 2005; Seeger et al., 2004). In addition, no obvious effect on neuronal morphology was observed in Hras KO mice (Manabe et al., 2000), but detailed analyses, as performed in the other studies are lacking. 
Although early pioneering studies indicated an involvement of $\mathrm{H}-, \mathrm{N}$ - and Kras in neurite outgrowth of PC12 cells (Bar-Sagi and Feramisco, 1985; Guerrero et al., 1986; Noda et al., 1985), small G proteins of the Rho family have long been regarded as the main factors that govern neuronal morphology (McAllister, 2000). The striking phenotypic changes seen in the Synras mouse, but also other findings (Jaworski et al., 2005; Kumar et al., 2005; Wu et al., 2001), changed this view. Ras signaling seems not only to be necessary for postsynaptic development, but also for activity dependent changes in spine morphology. Recently, spread of Hras activity from stimulated spines to nearby spines was observed, which leads to an increase in spine head volume in both (Harvey et al., 2008).

In addition to the involvement of Ras family proteins in regulating postsynaptic morphology, various Ras family $G$ proteins appear to play a role in neuronal polarization and in presynaptic development. Hras activity and signaling in axonal growth cones leads to symmetry breaking of cultured neurons, axonal outgrowth and branching (Fivaz et al., 2008; Yoshimura et al., 2006). Rras localizes exclusively to the developing axon and regulates axonogenesis, but does not play a role in dendrite morphogenesis (Oinuma et al., 2007; Saito et al., 2009). This is congruent with the developmental expression pattern of Rras, which shows a high concentration in immature neurons (Oinuma et al., 2007; Saito et al., 2009), but no expression in the adult brain (Allen_Mouse_Brain_Atlas, 2009; Nuñez Rodriguez et al., 2006).

\section{II.3.2. Ras Signaling and LTP}

In both the Synras and the Ras ${ }^{\mathrm{G} 12 \mathrm{~V}}$ mouse, LTP was found to be enhanced (Arendt et al., 2004; Kushner et al., 2005). Interestingly, KO of Hras has the same effect (Manabe et al., 2000). In organotypic slices, overexpression of constitutively active Hras leads to a reduction in LTP (Zhu et al., 2002), which was discussed as an occluding effect, i.e. basal transmission was too strong to achieve further potentiation. In another study that applied overexpression, wild type (wt) Hras led to reduced LTP implicating that Hras is a negative regulator of LTP.

The first electrophysiological study linking Ras signaling to LTP was in fact a KO study on Rasgrf1 (also known as cdc25NEF, RasGRF, GRF1), a Ras activator. The protein had been identified and cloned by three independent groups (Cen et al., 1992; Martegani et al., 1992; Shou et al., 1992) and found to activate H- and Nras (Shou et al., 1992), but also the Rho family G protein Rac1. After dissection of the 
Ras/Raf/Map2k/Mapk pathway and the presentation of molecular evidence for its involvement in LTP in 1996, the model still lacked a potent calcium sensor that would link calcium influx to Ras activation. Rasgrf1 contains an ilimaquinone motif that allows regulation by calcium/Calmodulin (Buday and Downward, 2008), making it a highly interesting candidate for the role of a calcium sensor in the Ras/Raf/Map2k/Mapk pathway.

Rasgrf1 KO mice show an impaired LTP in the amygdala, but not in the hippocampus. In addition to that, the mice exhibit a generally increased basic synaptic transmission in both regions (Brambilla et al., 1997). The combined phenotypes of increased basic synaptic transmission and impaired LTP were later often taken as an indication for an occluding effect, e.g. in the case of studies investigating Camk2a (Hayashi et al., 2000) or PSD-95 (officially known as Dlg4) (Stein et al., 2003).

Although the Rasgrf1 KO study is generally cited as evidence for a role of Ras proteins as positive regulators in LTP, the findings on basal transmission, the lack of an effect in hippocampal LTP and the possibility that Rac1 signaling could effectively trigger all the effects observed, are commonly neglected.

Following the "missing link in LTP" theory, an extensive electrophysiological study in 2006 investigated the interplay of Rasgrf1 and Rasgrf2 in the hippocampal CA1 region. The authors analyzed Rasgrf1 KO, Rasgrf2 KO and Rasgrf1/2 DKO (double knock out) mice and, based on their results, developed a model, in which Rasgrf1 acts predominantly on Mapk14 (p38a) via Rac1 and thus induces LTD, while Rasgrf2 acts via Mapk1 on LTP ( $\mathrm{Li}$ et al., 2006). This study has to be interpreted with caution for a number of reasons, the most striking being that Rasgrf1 and Rasgrf2 are not expressed together in the region examined. Rasgrf1 is expressed postnatally with protein levels reaching the adult state at P7. It is strongly expressed in the CA1 and CA3 region of the hippocampus. Immunohistochemical experiments reveal intense staining of the stratum pyramidale and dendrite gyrus (Wei et al., 1993). As Rasgrf1, Rasgrf2 is expressed postnatally (Tian et al., 2004), but is found in different regions than Rasgrf1. Its mRNA and protein are not found in the $C A 1$ and $C A 3$ region of the hippocampus, but are highly expressed in peripheral cortex layers (Allen_Mouse_Brain_Atlas, 2009; Fernández-Medarde et al., 2002). 
Based on controversial findings on Ras $G$ proteins and questionable results from analyses of Rasgrf1, the current literature cannot yet provide conclusive evidence for a definitive role of Ras signaling in LTP.

\section{II.3.3. Ras Signaling and AMPAR Trafficking}

Following the finding, that the Rasgrf1 $\mathrm{KO}$ mouse also displayed an increased basal transmission (Brambilla et al., 1997), researchers begun to investigate a putative role for Ras signaling in AMPAR ([alpha]-amino-3-hydroxyl-5-methyl-4isoxazolepopionic acid sensitive receptor) trafficking in the early 2000s. Hras was shown to have a positive effect on AMPAR transmission when overexpressed in hippocampal neurons (Zhu et al., 2002). Rap1a, also a member of the Ras G protein family, has the opposite effect on AMPAR transmission, and NMDAR transmission seems not to be affected by either of the proteins. This and several subsequent studies (Imamura et al., 2003; Kielland et al., 2009; Qin et al., 2005) used exogenous overexpression of G12V Hras mutants in the vast majority of experiments. The researchers concluded and further developed a model, according to which Ras G proteins have a positive effect on AMPAR trafficking and Rap proteins play an antagonistic role.

Also in the Synras mouse, basal transmission was found to be increased (Seeger et al., 2004). However, in the Ras ${ }^{\mathrm{G} 12 \mathrm{~V}}$ mouse, basal transmission appears to be unchanged (Kushner et al., 2005). The Hras KO mouse showed unchanged AMPAR transmission but an increased NMDAR transmission (Manabe et al., 2000). mEPSC (mini excitatory postsynaptic current) analysis in Synras and Ras ${ }^{\text {G12V }}$ mice, resulted in similar findings. mEPSC frequency is increased in both mouse models, whereas signal amplitude is unchanged as compared to control animals. These invivo findings contradict the findings from ectopic overexpression studies. Moreover, signaling of Hras in postsynaptic morphology was not taken into account by electrophysiological studies examining Hras signaling in AMPAR trafficking (Imamura et al., 2003; Qin et al., 2005; Zhu et al., 2002).

In summary, the role of Ras signaling in LTP and in basal transmission, in particular in AMPAR mediated transmission, remains largely unclear. 


\section{II.3.4. Postsynaptic and Presynaptic Effects of Ras Signaling}

In the Ras ${ }^{\mathrm{G} 12 \mathrm{~V}}$ mouse, paired-pulse facilitation (PPF), which is a electrophysiological protocol to examine presynaptic release probability, was found to be increased (Kushner et al., 2005). Together with an observed increase in mEPSC frequency and unchanged mEPSC amplitudes, this result would indicate a purely presynaptic effect of Hras, and was discussed as such by the authors (Kushner et al., 2005). In contrast, PPF was shown to be decreased in the Synras mouse (Seeger et al., 2004). These authors also discussed the combined phenotypes of increased mEPSC frequencies and unchanged amplitudes as a presynaptic effect, but as an indirect effect of postsynaptic strengthening. At least, both opinions are more or less in opposition to the idea of a solely postsynaptic function of Hras. This, along with the role of Hras in controlling neuronal morphology, is typically ignored in the studies on the role o Ras signaling in receptor trafficking (Kielland et al., 2009; Qin et al., 2005; Zhu et al., 2002).

The controversy as to whether effects of Hras are postsynaptically or presynaptically induced raises the question, where the protein is actually localized in neurons. In various transformed cell lines, $\mathrm{H}-, \mathrm{N}$ - and Kras are localized to the inner leaflet of the plasma membrane (Willingham et al., 1980). In neurons, $\mathrm{H}-, \mathrm{N}-$ and Kras are localized to ER, synaptic plasma membrane, but not to the nucleus, to synaptic vesicles or to the PSD, as revealed by immunohistochemical and biochemical methods (Mizoguchi et al., 1989). Hras signaling has been observed at the Golgi apparatus in PC12 cells (Bivona et al., 2003). Differential posttranslational modification (i.e. prenylation and palmitoylation) primarily targets Kras to endomembranes, whereas $\mathrm{H}$ - and Nras can also be trafficked to the plasma membrane (Choy et al., 1999). Hras, but not K- or Nras were detected in the NMDAR complex (Husi et al., 2000) and both $\mathrm{H}$ - and Kras were found in the "PSD proteome" (Cheng et al., 2006), albeit not among the 32 core proteins. Kushner et al. (2005), showed a partly overlapping Hras and VGLUT1 staining in hippocampal sections and assumed Hras to be presynaptically localized, in accordance with the effects of Hras observed in their Ras ${ }^{\mathrm{G} 12 \mathrm{~V}}$ mouse line (Kushner et al., 2005).

Recently, imaging studies assessed Ras activation and mobility in the plasma membrane of single dendritic spines using Ras sensitive FRET (fluorescent resonance energy transfer) sensors (Harvey et al., 2008; Yasuda et al., 2006). Also using Ras sensitive FRET sensors, Hras activity was detected in axonal growth 
cones of developing neurons (Fivaz et al., 2008). Beyond these studies, conclusive and comparative studies that apply modern methods to investigate the exact subcellular localization of small G proteins of the Ras family in neurons, are lacking. Knowledge from other cell types suggests that also in neurons, Ras proteins are found throughout the cell, with a concentration at the Golgi apparatus, ER and plasma membrane.

\section{II.4. Complexity of Ras Signaling}

The use of G12V Hras has revealed a vast number of pre- and postsynaptic effects of Ras signaling. Interestingly, the results of the different studies applying mutated Hras cannot be brought into accord in the majority of cases; basically, each line of research follows an independent dogma. The cause of the controversies seems to be the expression levels of Hras and the levels of activation in the different experimental systems. Indeed, Kusher et al. (2005) argue, that the striking difference in the phenotypes of Ras ${ }^{\mathrm{G} 12 \mathrm{~V}}$ and Synras mice is due to relatively different expression levels of G12V Hras. The Synras mouse shows higher G12V Hras expression than the Ras ${ }^{\mathrm{G} 12 \mathrm{~V}}$ mouse, implying that the stronger the availability of activated Hras, the stronger the effect on the postsynapse. This would also be congruent with the studies providing evidence for a role of Ras signaling in AMPAR trafficking (Kielland et al., 2009; Qin et al., 2005; Zhu et al., 2002), since the exogenous expression of G12V Hras that was used in these studies, can be expected to result in very high expression levels.

Which effect-causing mechanisms are that sensitive to the availability of activated Hras? Ras proteins regulate a variety of downstream effectors that most likely exhibit different affinities for activated Ras and govern different effect-causing mechanisms. Many recent studies have attributed the pleiotropic effects of Hras solely to the different downstream pathways it activates (Jaworski et al., 2005; Kumar et al., 2005; Yoshimura et al., 2006).

Not only chemical affinity but also spatial proximity governs Ras pathway selection. Subcellular proximity of $G$ proteins and their effectors determines the molecular pathway that is activated and specifically triggers one effect. It is clear that the subcellular distribution of overexpressed proteins such as G12V Hras might differ significantly from that of their endogenous counterparts. Mislocalization of overexpressed protein can account not only for abnormal additional signaling, but 
also for abnormal absent signaling. For example, Hras G12V is excluded from plasma membrane lipid rafts and only interacts with its membrane resident effectors (Karnoub and Weinberg, 2008).

The complexity of Ras downstream signaling that causes the extraordinary sensitivity to experimental perturbation is described in the next sections.

\section{II.4.1. Multitude of Ras Downstream Pathways}

The Pi3k pathway was the first effector pathway of Ras identified besides the classical Raf/Map2k/Mapk pathway. The Pi3k family is a group of heterodimeric enzymes composed of a catalytic (known as Pik3c or p110) and a regulatory (known as Pik3r or p85) subunit. Hras has been shown to bind the catalytic subunit and to thereby regulate Pi3k function directly (Rodriguez-Viciana et al., 1994).

Over the years, several other Ras effectors have been described, which include a number of proteins with diverse roles in cell physiology, such as phospholipase $\mathrm{C}-\varepsilon$ (Plce), T-cell lymphoma invasion and metastasis-1 (Tiam1), Ras interaction/interference protein-1 (Rin1), Mllt4 (also called Afadin or AF-6), and the Ras association domain-containing family of proteins (Rassf). Some effects, that were previously thought to be triggered by the classical Raf/Map2k/Mapk pathway, have since been revisited and attributed to these new effectors. Antiapoptotic effects were found to depend on the Pi3k pathway mostly; some effects in differentiation depend on the Ralgds pathway more than the Mapk pathway (Karnoub and Weinberg, 2008). Ralgds is an example of cross talk within the Ras family. It is an activator for the Ras $\mathrm{G}$ protein Rala and Ralb and therefore, e.g. Hras can activate Rala. Rala and Ralb then signal via Ralbp1 (Ral binding protein 1), phospholipase D (Pld) and filamin (Takai et al., 2001). Mllt4 contains both microtubule and actin binding motifs and was shown to associate with proteins that are involved in regulating cell polarity (Mandai et al., 1997), indicating that Ras might act on cytoskeletal dynamics via this pathway. Rin1, which is an activator of Rab5 like proteins, can trigger endocytosis, and Ras signaling was shown to participate in this process (Tall et al., 2001). The less well-studied Rassf proteins seem to be involved in regulation of apoptosis (Karnoub and Weinberg, 2008).

Sequence similarities in the Ras binding domains of all these effectors indicate that there might still be more target proteins that are regulated by Ras (Goldfinger, 
2008). In neurons however, all pathways besides the Mapk, Pi3k and Ralgds pathways have been studied only cursory so far.

\section{II.4.2. Shared Downstream Pathways}

Apart from diverging pathways downstream of Ras, signaling from other small $G$ proteins can converge upon Ras triggered pathways. Based on direct evidence and sequence similarity, all of the Ras family G proteins except Rala and Ralb are thought to share many downstream effectors. However, small differences in protein sequence and subcellular localization, likely result in different affinities and differential activation of effectors. For example, Kras is a more potent Raf/MEK/Erk activator than Hras, and in turn Hras is a more potent Pi3k activator (Hancock, 2003). Activated Rras only weakly binds Raf1, and similarly, Rap1a affinity to and activation of Raf1 are distinct from those of Ras (Goldfinger, 2008).

In addition to pathways shared by Ras family G proteins, some pathways are even shared by the whole superfamily of small $G$ proteins. Rho family proteins can signal via the Pi3k pathway and indirectly via the Mapk pathway (Takai et al., 2001). Rab5 and Rab7 of the Rab family can also signal via Pi3k (Stenmark, 2009), and Rala and Ralb regulation by Ras/Ralgds leads to activation of Rho $\mathrm{G}$ proteins via Ralbp1 (Takai et al., 2001). With knowledge of this extensive cross talk, it is often not possible to attribute specific mechanisms to one family, let alone one protein within the superfamily of small $G$ proteins.

Diverging pathways downstream of identified Ras effectors complicate the situation even further. For example, Mapk was first reported in insulin-stimulated 3T3-L1 adipocytes and in epidermal growth factor (Egf) stimulated fibroblasts as a protein that phosphorylates the microtubule associated protein 2 (Map2), and was therefore first designated Map2 kinase (Ahn, 1993; Hoshi et al., 1988; Ray and Sturgill, 1987). Mapk also directly associates with Map2 and therefore with the cytoskeleton, and phosphorylation of Map2 by Mapk has a significant impact on microtubule stability (Sánchez et al., 2000). Map2 is present in dendrites and a major factor in dendrite stability (Bernhardt and Matus, 1984; Matus, 1994). However, the Mapk pathway also leads to activation of the transcription factor Creb1 (cAMP responsive element binding protein 1), resulting in Wnt2 (wingless-related MMTV integration site 2) expression and secretion. Wnt2 in turn is an extracellular signal for dendrite arborization (Wayman et al., 2006). Attributing one of these possible 
mechanisms by which Mapk can regulate dendrite morphology to a specific Ras G protein is very difficult.

In an experiment, the described sharing of downstream pathways such as the Pi3k pathway complicates the interpretation, which $G$ protein triggered an observed effect. For example, results acquired in experiments that not involved Ras and that showed an effect of the Pi3k pathway in dendrite morphogenesis (Jaworski et al., 2005; Kumar et al., 2005) could as well be attributed to other upstream factors (e.g. Rab G proteins).

\section{II.4.3. Pathway Selection by Ras G Proteins}

How is it decided, which signaling pathway is selected by a specific Ras protein? In general, this choice is determined by the availability, i.e. existence, physical proximity and state (active/inactive) of the signaling pathway involved. Availability of signaling components is regulated by developmental stages, tissue and cell specific expression, subcellular localization to organelles and scaffolding complexes, and temporal dynamics e.g. temporal dynamics, transport, and half-life of activation. An example for developmental selection of signaling pathway components is Nras. It is expressed in early stages of brain development only, whereas Kras and Hras expression is stable or even increases during development, and both are present in adult tissue. (Allen_Mouse_Brain_Atlas, 2009; Leon et al., 1987)

Tissue and cell specific expression of Ras proteins has not been studied in detail. However, Hras is only present in neurons within the brain (Mizoguchi et al., 1989), while other Ras proteins such as Mras have been found to be present in astrocytes as well (Nuñez Rodriguez et al., 2006).

Within the cell, differential palmitoylation leads to trafficking of $\mathrm{H}$ - and Nras to the endoplasmatic reticulum (ER), Golgi and plasma membrane, whereas Kras is only present at ER and plasma membrane. The plasma membrane itself contains microdomains that are specified by their lipid composition. The best characterized of these microdomains are caveolae and lipid rafts. In both, Hras and all the components of the Raf/Map2k/Mapk pathways have been found. Kras is found predominantly in non-raft plasma membrane, indicating a differential pathway selection by this protein (Hancock, 2003; Omerovic and Prior, 2009).

Where exactly Hras activation takes place, is still a matter of debate. Using overexpression techniques, activation was shown to occur at the Golgi (Bivona et al., 
2003), but more recent findings support the classical view of predominant Hras activation at the plasma membrane (Augsten et al., 2006). Neurons are highly compartmentalized cells that contain Golgi and ER structures not only within their somata. Dendrites in particular contain large numbers of endomembranous structures, among them Golgi outposts, smooth endoplasmatic reticulum and mitochondria. Within spines, neurons contain a specific organelle known as the spine apparatus. Functionally, all these organelles are thought to act in protein trafficking, but some also in local protein synthesis (Kennedy and Ehlers, 2006).

Several proteins selectively bind Ras proteins and components of downstream pathways and thereby facilitate signaling via the components that form one complex. For example, Iqgap (IQ motif containing GTPase activating protein) isoforms are widely expressed and bind directly to B-Raf, Map2k and Mapk, bringing all these signaling components in close proximity (Brown and Sacks, 2009). The neuronal specific protein Ksr1 (kinase suppressor of ras 1), which is located in somata and apical dendrites of pyramidal neurons, switches Egf signaling from a proliferative signal to a differentiation signal in PC12 cells (Müller et al., 2000).

\section{II.5. Control of Ras Signaling}

Given the enormous complexity of Ras signaling pathways and the extensive cross talk between these pathways, general perturbation of Ras signaling cannot reveal specific effects, e.g. by dominant active mutants of Ras proteins. This is due to the fact, that it is essentially impossible to correctly choose the right developmental stage, the tissue or culture system that contains only a specific and restricted set that can be studied in isolation. Likewise, no expression system can guarantee the appropriate expression levels of exogenous protein (e.g. Ras mutants) that would then have the correct subcellular localization and physiological affinity to scaffolds and effectors. In this context, many of the effects found in studies employing overexpression of G12V Hras must be considered as a result from ectopic signaling and artifactual activation of cross talk. Consequently, many findings on Hras cannot be taken at face value and attributed to Hras alone, but must be seen as effects of small $G$ protein signaling in general. They have to be attributed to other, related Ras proteins and/or downstream pathways that were activated in the wrong place at the wrong time and to a wrong degree. 
Above considerations lead to the question as to whether Ras proteins themselves are the best starting point for analysis of Ras signaling. A physiological process with such a huge range of possible implications inevitably complicates the interpretation of all the effects caused by general perturbation in a given experimental situation. Essentially, signaling by Ras $G$ proteins must be seen as a pluri-potent and omni-present activity within the cell. Any given cell must very stringently select activation of these proteins and the spatially and temporally segregated downstream pathways to avoid unnecessary or even harmful effects of Ras signaling, such as tumorigenesis.

Indeed, nature orchestrates the pleiotropic effects of Ras proteins very efficiently. The molecular correlates that conduct this regulation are guanine nucleotide exchange factors (GEFs) and GTPase activating proteins (GAPs). GEFs catalyze the release of GDP by reaching into the nucleotide binding site of small $G$ proteins and opening it, allowing GDP to escape and GTP to bind. This is achieved by the Cdc25-like catalytical domain of GEFs, also referred to as "Ras GEF domain" (Boriack-Sjodin et al., 1998; Ehrhardt et al., 2002; Stryer et al., 2002). GAPs interact with small $G$ proteins and increase their intrinsic GTPase activity, leading to a much faster termination of $G$ protein activation.

These proteins typically exhibit rather high substrate specificities, i.e. they only activate/inactivate only a small subset of Ras G proteins each. Mostly, GEF and GAP specificities are restricted towards a few members of one Ras subfamily. In some cases, the specificity crosses the borders of subfamilies, but only very few GEFs and GAPs show specificity for members of more than one family (which is then achieved by separate domains). A second level of specificity is provided by the often very restricted subcellular localization of GEFs and GAPs, a feature that distinguishes them from their more widely distributed substrates.

In view of these characteristics, Ras GEFs and GAPs provide a very useful alternative starting point for analysis of the functional role of Ras signaling.

\section{II.5.1. Specificity of Ras GEFs}

GEFs that activate classical Ras $G$ proteins in the brain belong to three families, the Sos, Rasgrf and Rasgrp family. In case of the Rasgrf and Rasgrp proteins, an activity towards the Rras/Mras subfamily besides the classical Ras $G$ proteins seems to be a general pattern of specificity. The activity of Sos GEFs seems to be restricted 
towards the classical Ras G proteins and Mras, while Rras or Rras2 are not affected. Rasgrf and Sos proteins not only contain a Ras GEF domain, but also a Rho GEF domain, making them parallel activators of Rac $G$ proteins of the Rho family (Bos et al., 2007).

Apart from these three GEF families, members of the Rapgef family can act on classical Ras and Rras/Mras proteins under some circumstances. Rapgefs are well investigated GEFs of Rap1 and Rap2 proteins. However, Rapgef1 (also known as C3G) and Rapgef3 (also known as Epac1) were shown to also act directly on Rras (Gotoh et al., 1997; López De Jesús et al., 2006; Roscioni et al., 2008), and for Rapgef2 (also known as CNrasGEF, nRap-GEF, PDZ-GEF), an activity towards Hras has been observed (Pham et al., 2000). However, it is still unclear if this is a matter of direct or an indirect interaction (Kuiperij et al., 2003).

Another well-known protein that has been considered a Ras GEF is phopholipase $\mathrm{C}$ epsilon (Plce). It contains a Ras association (RA) domain and a Cdc25-like domain at its N-terminus. However, it is not clear if the Cdc25 domain of Plce is functional and if Plce is a potent GEF for classical Ras or for Rap proteins (Buday and Downward, 2008; Jin et al., 2001; Lopez et al., 2001). Interestingly, Plce is also a downstream effector of Ras G proteins (see chapter II.4.1).

A more recently described Ras GEF is Kndc1 (kinase non-catalytic C-lobe domain (KIND) containing 1, also known as VKIND). The only known substrate of this GEF is Hras (Huang et al., 2007; Mees et al., 2005).

\section{II.5.2. Roles of Ras GEFs in the Brain}

Sos1 (son of sevenless homolog 1) was the first Ras GEF discovered and along with its discovery, the classical model of Ras regulation by extracellular growth factors was established. According to this model, growth factors induce a rapid dimerization and autophosphorylation of their receptors. The resulting phosphotyrosine residues function as binding sites for the $\mathrm{SH} 2$ (Src homology 2) domain in Grb2 (growth factor receptor-bound protein 2). Grb2 in turn, recruits Sos1, subsequently leading to activation of Ras proteins. Sos1 mRNA is ubiquitously expressed in brain, with the highest concentration found in hippocampus and cerebellum (Allen_Mouse_Brain_Atlas, 2009). The protein was detected in the PSD by electron microscopy (EM) and biochemical purification (Suzuki et al., 1999). No 
electrophysiological data on the function of Sos1 exist so far, most likely because a KO of Sos1 in mice causes the animals to die before birth (Wang et al., 1997).

Sos2 protein expression has not yet been investigated in detail. Its mRNA is found in all regions of the brain, but it is not enriched in a specific region as seen for Sos1 (Allen_Mouse_Brain_Atlas, 2009). In contrast to Sos1, Sos2 is not present in purified PSD fractions (Suzuki et al., 1999). Sos2 KO mice are viable and apparently healthy (Esteban et al., 2000), and electrophysiological data indicate that LTP is not affected in these animals (Arai et al., 2009). Other physiological properties besides LTP have not been tested yet.

Rasgrf1 has already been mentioned as the potential missing link that relays calcium influx into the postsynapse to Hras and the Mapk pathway (see chapter II.3.2). However, Rasgrf1 might also function in general neuronal excitability, as KO mice show an AMPAR- and NMDAR- independent increase in action potential generation (Tonini et al., 2001). Moreover, long-term depression (LTD) is reduced in Rasgrf1 $\mathrm{KO}$ animals ( $\mathrm{Li}$ et al., 2006). In the hippocampus, Rasgrf1 is highly expressed in the CA1 and CA3 regions and in the dentate gyrus (Wei et al., 1993). Rasgrf1 is expressed in neurons where it is present in punctuate stainings along and within dendrites and cell bodies, and it can be detected in purified PSD fractions (Sturani et al., 1997; Zippel et al., 1997). However, the exact localization of Rasgrf1 in neurons has not been determined by colocalization studies or EM analyses. Experiments on PC12 cells indicate that upon activation by Rasgrf1, both Ras and Rho G proteins act in concert to control cell morphology (Yang and Mattingly, 2006).

Like Rasgrf1, Rasgrf2 is expressed postnatally (Tian et al., 2004), but found in different regions than Rasgrf1. Its mRNA and protein are not found in the CA1 and CA3 region of the hippocampus, but rather in peripheral cortex layers (Allen_Mouse_Brain_Atlas, 2009; Fernández-Medarde et al., 2002). The subcellular localization of Rasgrf2 has not been investigated yet.

The family of Rasgrps (Ras guanyl releasing proteins) contains four genes encoding at least five proteins; four of them are expressed in the brain. These are Rasgrp1, Rasgrp2a, Rasgrp2b and Rasgrp3.

Rasgrp1 mRNA and protein are highly expressed in the brain. It is found in the olfactory bulb, cortex, caudo-putamen (including striatum), hippocampus and thalamus, but only at very low levels in midbrain, cerebellum, pons and medulla (Allen_Mouse_Brain_Atlas, 2009; Pierret et al., 2000; Toki et al., 2001). Expression 
of Rasgrp1 starts in late embryonal stages (E18) in caudo-putamen and subiculum and reaches its adult pattern at P21 (Pierret et al., 2001; Toki et al., 2001). Within the hippocampus, the Rasgrp1 protein is found from P2 on only in the CA1 and CA3 region, but not in the dentate gyrus. Immunohistochemical experiments followed by EM detection indicate a localization of Rasgrp1 exclusively in the somatodendritic compartment of pyramidal neurons (Pierret et al., 2001).

The Rasgrp2 gene expresses two isoforms. The first one discovered was called Caldag-gefl, while the second isoform was named Rasgrp2, in accordance with the human genome project nomenclature committee (Clyde-Smith et al., 2000). To omit confusion of the reader, I will refer to the encoded proteins as Rasgrp2a and b, respectively, and to the shared gene or mRNA as Rasgrp2. Rasgrp2b is the longer isoform of the both, additionally containing a $\mathrm{N}$-terminal acetylation site that targets Rasgrp2b to the membrane. This is a unique feature among the Rasgrp proteins.

In contrast to the other members of the Rasgrp family, Rasgrp2a shows GEF activity towards Rap1a, Rap2a, Rras and Rras2 (TC21), but not towards H-, N-, Kand Mras.

Rasgrp2b however shows additional activity towards $\mathrm{N}$ - and Kras, but not to Hras, which might be due to the plasma membrane targeting of the protein (ClydeSmith et al., 2000). Rasgrp2 mRNA is already present at small levels in the embryonic brain and expression peaks at P3. At this time point, the mRNA is present throughout the brain, including cerebellum (Toki et al., 2001). Until P14 however, mRNA levels decline in most regions, until the caudo-putamen remains the only region expressing Rasgrp2 mRNA (Allen_Mouse_Brain_Atlas, 2009; Toki et al., 2001). For Rasgrp2a, this adult mRNA expression pattern was immunohistochemically confirmed (Kawasaki et al., 1998). The cellular and subcellular localization of Rasgrp2a and $b$ in neurons is not known yet. However, since Rasgrp2 and Rasgrp1 mRNA were found to colocalize in the striatum, an expression of Rasgrp2 in neurons is likely (Toki et al., 2001). Rasgrp2 KO mice display impaired integrin signaling via Rap1a and perturbed cell adhesion of leukocytes and platelets (Bergmeier et al., 2007).

Rasgrp3 is expressed in the brain and an early immunohistochemical study finds it to be most dominantly expressed in cerebral and cerebellar white matter (Yamashita et al., 2000), indicating a preferred expression in oligodendrocytes. Data on region specific expression and detailed investigations of cellular and subcellular 
localization are lacking. Interestingly, Rasgrp3 has the broadest substrate specificity of all Ras GEFs, since it activates not only Hras, Mras, Rras and Rras2 (TC21), but also Rap1a (Ohba et al., 2000; Rebhun et al., 2000; Yamashita et al., 2000). Rasgrp4 is not expressed in the brain (Reuther et al., 2002; Yang et al., 2002) and therefore will not be considered in this text.

As mentioned above, the catalytic activity of Rapgef2 towards Hras is still disputed. However, Rapgef2 is a neuron specific protein highly expressed in hippocampal pyramidal cells, where it is mostly found in somatodendritic compartments (Bilasy et al., 2009). KO mice die during embryogenesis (Wei et al., 2007) and a telencephalon specific conditional KO (cKO) of Rapgef2 exhibits a severely aberrant cortex structure (Bilasy et al., 2009). A putative function of Rapgef2 in synaptic transmission and plasticity has not been investigated yet.

Kndc1 seems to be weakly expressed in the hippocampus. A stronger expression can be found in the cerebellum. In hippocampal pyramidal neurons, Kndc1 is expressed in the somatodendritic compartment, and in cultured neurons, it partly colocalizes with Map2. Moreover, Kndc1 was shown to be a negative regulator of dendrite development in cultured neurons (Huang et al., 2007). Electrophysiological experiments to investigate further roles of Kndc1 have not been performed yet.

Gripap1 (GRIP1 associated protein 1, also known as GRASP-1) was previously reported as a Ras GEF and to be involved in AMPAR trafficking by binding to the AMPAR/Grip1 complex (Ye et al., 2000). However, recently Gripap1 was shown to be a Rab3 GEF and not to display activity towards Ras family $G$ proteins (Hoogenraad et al., 2010).

\section{II.5.3. Roles of Ras GAPs in the Brain}

The best-investigated Ras GAPs in the brain are Syngap1 and Neurofibromin1 (Nf1). Syngap1 exhibits a dual specificity towards Ras and Rap subfamilies (Yarwood et al., 2006). It is expressed postnatally with a peak of expression at P35 (Porter et al., 2005). It was found to be highly expressed in the postsynapse (Chen et al., 1998), and initially thought to be exclusively localized to excitatory postsynapses. Proteomics studies indicated that the levels of Syngap1 at the PSD are as high as those of the major PSD scaffolds PSD-95 and PSD-93 (officially known as Dlg2) combined (Cheng et al., 2006). However, a recent comparison of the localization of 
different Syngap1 isoforms revealed that at least one isoform is present at some inhibitory postsynapses as well (Moon et al., 2008). Syngap1 KO animals die during embryonal development. In the hippocampus of heterozygous KOs synaptic transmission is normal, but LTP is reduced (Kim et al., 2003). Homozygous KO neurons, however, show increased basal transmission and increased spine density (Vazquez et al., 2004). Another study confirmed this electrophysiological phenotype, but concluded that it is due to increased glutamate receptor surface expression, and not to a change in spine morphology (Rumbaugh et al., 2006). A more recent study showed that LTD is impaired and spine density is increased in hippocampi of heterozygote Syngap1 KOs (Carlisle et al., 2008). As for its downstream target, Ras, a role in spine morphology s still a matter of controversy.

Nf1 is a protein in which mutations can lead to cancer syndromes. These become manifest as accumulations of pigmented lesions and sporadic malignant outgrowths, such as neurobfibromas or neurofibrosarcomas (Neurofibromatosis type1) (Karnoub and Weinberg, 2008). Nf1 activity is clearly specific for the Ras subfamily (Yarwood et al., 2006). It is found throughout the brain in neuronal as well as in non-neuronal cells. In neurons, Nf1 localizes to cell bodies and apical dendrites of pyramidal cells (Allen_Mouse_Brain_Atlas, 2009; Nordlund et al., 1993; Zhu and Parada, 2001). In a detailed EM study, Nf1 was found at the smooth endoplasmatic reticulum (SER), but not at the Golgi, mitochondria, nucleus, nuclear envelope or plasma membrane of neurons (Nordlund et al., 1993). Biochemical studies indicate that Nf1 is part of the NMDAR complex (Husi et al., 2000). Nf1 KO mice die as embryos and therefore only heterozygous animals were investigated in electrophysiological experiments so far. They show a slightly reduced basal synaptic transmission, reduced LTP, but enhanced GABAergic inhibition due to increased presynaptic vesicle release (Costa et al., 2002; Cui et al., 2008; Guilding et al., 2007). The authors speculate that the impaired LTP is due to the increased inhibition in these animals.

Rasa and Rasal GAPs are less well-studied proteins in neurobiology, but their expression patterns as well as recent data make them interesting subjects for future research. Rasa1 (also known as p120GAP, Rasa, RasGAP, GAP) is ubiquitously expressed in the brain (Allen_Mouse_Brain_Atlas, 2009), but its cellular and subcellular expression has not been studied in detail. However, besides Syngap1, it is the only Ras GAP found in the PSD proteome (Cheng et al., 2006). It stimulates 
Hras and Rras, shows no activity towards Rho or Rap (Nakamura et al., 2005; Yarwood et al., 2006), and was recently found to act in axonogenesis via Ras subfamily proteins (Endo and Yamashita, 2009).

Rasa3 (also known as Gaplll, GAP1(IP4BP), R-Ras GAP) was discovered in 1995 (Baba et al., 1995; Cullen et al., 1995) and displays activity towards Hras, Rras, Rap1a, but not towards Kras, Rac1 or Rap3a. Rasa3 mRNA is expressed in neurons and oligodendrocytes of the hippocampus, cortex and cerebellum and peaks at P35. Immunohistochemical analysis revealed high protein levels in the CA1 region of the hippocampus, but low protein expression in CA3 and dentate gyrus. At the subcellular level, Rasa3 is found in cell bodies (endomembranes and plasma membrane, less in the cytosol) and proximal apical dendrites (Signore et al., 1999).

Little is known about Rasa2 (also known as mGAP1, GAP1m) and Rasa4 (also known as Capri), whose mRNA seem to be more or less exclusively expressed in the olfactory bulb (Allen_Mouse_Brain_Atlas, 2009). Similarily, Rasal1 (also known as MRASAL) and Rasal2 (also known as NGAP) are not characterized in a neuronal context yet. Their mRNA expression pattern in the brain is interesting due to the fact that they seem to be present only in the telencepalic cortex. In the hippocampus, only the CA1 region seems to express Rasal1 and Rasal2 (Allen_Mouse_Brain_Atlas, 2009). The putative Ras GAP Dab2ip is expressed postnatally and highly concentrated in hippocampal pyramidal neurons (Chen et al., 2006; Homayouni et al., 2003). Iqgaps 1-3 contain RasGAP domains (Bos et al., 2007). They may be involved in Mapk- pathway scaffolds (Brown and Sacks, 2009), but their role as Ras GAPs in the brain has not yet been investigated.

\section{II.5.4. Interplay of Ras Regulatory Proteins in the Hippocampus}

The rodent hippocampal formation includes the "retrohippocampal region" comprised of the entorhinal cortex and the subicular complex (subiculum, parasubiculum, presubiculum) and the "hippocampal region" comprised of hippocampus proper (Ammon's horn = Cornu ammonis regions CA1, CA2 and CA3 fields) and the dentate gyrus (Abrams, 2005; Amaral and Witter, 1989; Burwell et al., 1995). The processing of information in the hippocampus takes place in a corticohippocampal-cortical loop. Cortical input entering the superficial layers II and III of the entorhinal cortex is relayed directly to the CA3 region. Processes of pyramidal 
neurons in the CA3 region form the Schaffer collateral fibers that pass the neuronal cell body-free CA2 region and terminate at pyramidal neurons in the CA1 field.

The CA1 region is the most thoroughly investigated region in the brain. The main reason for this is its physiological importance for learning and memory formation. Secondly, it is a distinctly layered structure, which makes it nicely suitable for electrophysiological and optical investigations. In a dorso-ventral order, it comprises of four regions, the stratum oriens (so), the stratum pyramidale (sp), the stratum radiatum (sr) and the stratum lacunosum moleculare (slm). The stratum pyramidale contains the somata of excitatory pyramidal and inhibitory interneurons. Pyramidal neurons extend their basal dendrites and their axons into the stratum oriens and their thicker and longer apical dendrites into the stratum radiatum. The apical dendrites branch several times in the stratum radiatum. In the stratum lacunosum moleculare they end in so called "dendritic tufts". Most incoming signals are therefore received in the stratum radiatum and the stratum lacunosum moleculare, signal integration takes place in the stratum pyramidale and conduction of action potentials occurs through the stratum oriens (Spruston, 2008).

Of the six proteins within the classical Ras protein subfamily and the Mras/Rras subfamily, three proteins are expressed in the adult CA1 region of the brain. These are Hras, Kras and Mras. In total, at least 12 regulatory proteins (or their mRNA) for these small $G$ proteins have been detected in the CA1 region. However, cellular and subcellular localizations have not been determined for most of them. The neuronal GEFs expressed in the CA1 are Sos1, Rasgrf1 and Rasgrp1. Whether Sos2 and Rasgrp3 are also present in neurons remains to be determined. Ras GAPs that have been identified in CA1 neurons include Syngap1 and Nf1, Rasa1 and Dab2ip. In addition, Rasal1, Rasal2 and lqgap1 may also be present in the CA1 region. Of these Ras regulators Syngap1 and Sos1 are clearly localized to the postsynapse. No Ras regulatory protein that would be specifically localized to the presynapse has been found yet. 


\section{II.6. Rasgrp1}

\section{II.6.1. Rasgrp1 in Non-neuronal Functions}

Rasgrp1 is a key Ras regulator. Despite of its strong expression in the brain, research has so far have concentrated on its function in the haematopoietic system. Rasgrp1 has been found to link T-cell receptor (TCR) signaling to Hras in a diacylglycerol (DAG) dependent manner (Ebinu et al., 2000). Hras activation is necessary for differentiation of double negative thymocytes (CD4 / CD8 $)$ into double positive thymocytes $\left(\mathrm{CD} 4^{+} / \mathrm{CD}^{+}\right)$. Only double positive thymocytes are able to undergo positive selection into single positive cells (CD4 / $\mathrm{CD}^{+}$or $\left.\mathrm{CD}^{+} / \mathrm{CD} 8^{-}\right)$, which are ready to differentiate into killer or helper cells. Accordingly, Rasgrp1 KO mice exhibit a defect in positive selection (Dower et al., 2000; Priatel et al., 2006).

Interestingly, the GEFs Rasgrp1 and Sos $1 / 2$ functionally cooperate in lymphocytes to integrate TCR signals. Rasgrp 1 modulates Sos $1 / 2$ by providing GTP bound Ras (Ras-GTP) to the allosteric Ras-binding pocket of Sos $1 / 2$, leading to an increased sensitivity of Sos $1 / 2$ and increased Ras signaling. This positive feedback loop may be a form of molecular short-term memory (Chakraborty et al., 2009).

Rasgrp1 might also play a role in systemic lupus erythematosis (SLE), which is characterized by dysregulated signaling in T-and B-lymphocytes (Peng, 2009). In the kidney nephron, Rasgrp1 is involved in the regulation of sodium reabsorption by preventing surface expression of sodium chloride transporter (NCC) (Ko et al., 2007).

\section{II.6.2. Insights in the Function of Rasgrp1 in the Brain}

In an investigation of memory formation in the subregions of the rat hippocampus that involved spatial memory training and subsequent microarray based gene expression profiling, Camk2a, Neuroligin1 and Rasgrp1 mRNA expression was shown to be strongly upregulated in the CA3 region of the hippocampus upon this spatial memory training (Haberman et al., 2008), indicating that Rasgrp1 may play a role in memory formation.

Rasgrp1 may also be involved in motor side effects induced by certain antiparkinsonian therapies. Under normal conditions, Rasgrp1 expression in the striatum is rather low and mainly restricted to striasomes (also called "patches"). In contrast, Rasgrp2 is expressed abundantly in the striatum and only found in the complementary compartment, i.e. the striatal matrix. Parkinson's disease is characterized by loss of dopaminergic neurons in the striatum, which leads to motor 
dysfunction. Patients treated with L-DOPA first experience a gain in motor function, but suffer dyskinesia in prolonged treatment. In a parkinsonian rat model treated with L-DOPA, Rasgrp1 was found to be upregulated in the striatum, with a concomitant downregulation of Rasgrp2. These high Rasgrp1 and low Rasgrp2 mRNA levels quantitatively correlated with high dyskinesia scores, indicating that Rasgrp1 and Rasgrp2 may be involved in general motor activity control (Crittenden et al., 2009).

\section{II.6.3. Structure of Rasgrp1 and Mechanisms of Function}

Rasgrp proteins share a high degree of structural similarity. Most $\mathrm{N}$-terminally, they exhibit a Ras exchanger motif (REM), which is a common structural feature found in many Ras GEFs, and the catalytical Ras-GEF domain. Towards the Cterminus, two EF-hand (EFh) motifs and a phorbol esters/diacylglycerol binding (C1) domain follow. These domains mediate regulation by calcium and DAG, respectively, which both activate Rasgrp1 (Dower et al., 2000; Ebinu et al., 1998; Ebinu et al., 2000; Kawasaki et al., 1998; Lorenzo et al., 2001; Reuther et al., 2002).

One mechanism to achieve specificity in Ras signaling is the differential subcellular targeting of regulatory and downstream components of the signaling pathway. Localized signaling of Rasgrp1 was demonstrated by the finding that overexpression of Rasgrp1 in a number of cell lines activates Hras selectively on the Golgi apparatus, leading to neurite outgrowth in PC12 cells (Bivona et al., 2003). Although this finding has later been revised by evidence for a predominant activation of endogenous Hras at the plasma membrane (Augsten et al., 2006), it provided insights into general mechanisms of compartmentalized Ras signaling. Of particular importance for the mechanism of compartmentalized Ras signaling through Rasgrp1 is the $\mathrm{C} 1$ domain, because it mediates translocation of Rasgrp1 from the cytosol to the Golgi apparatus (Bivona et al., 2003). The second messenger DAG is produced by the cleavage of phosphatidyl inositol-bisphosphate $\left(\mathrm{PIP}_{2}\right)$ into inositoltrisphosphate $\left(\mathrm{IP}_{3}\right)$ and DAG. As PIP 2 , DAG remains incorporated in the membrane. Therefore, proteins containing a C1 domain, which binds DAG often show a high affinity to membranes. The $\mathrm{C} 1$ domain however, seems not to be the only structural feature of Rasgrp1 that determines its subcellular localization. Other findings indicated that a plasma-targeting domain (PT) and a suppressor of plasma targeting domain (SuPT) domain within the C-terminus of Rasgrp1 compete or cooperate with the C1 domain to shuttle Rasgrp1 between cellular compartments (Beaulieu et al., 
2007). In addition, the first EF hand and the Ras-GEF domain also seems to be involved in the translocation of Rasgrp1 to the plasma membrane (Tazmini et al., 2009).

Neurons exhibit the highest degree of compartmentalization among all known cell types. In view of the complex subcellular compartmentalization of Rasgrp1 and its regulation by key second messengers, along with the fact that Rasgrp1 expression parallels the developmental increase in neuronal polarization and complexity, I set out to test the possibility that Rasgrp1 is involved in nerve cell differentiation and function.

\section{II.7. Aims of the Study}

The aim of this study was to characterize the Rasgrp1 KO in order to gain insights into the role of Rasgrp1 in differentiation and function of the brain. As Rasgrp1 is strongest expressed in the hippocampus, this study concentrated on this region of the brain. Since spatial control has a strong impact on the function of Ras signaling, the localization of Rasgrp1 in the hippocampal CA1 region was studied in detail. Fluorescent immunohistochemistry was used to label Rasgrp1 and to compare its staining pattern with that of cellular and subcellular markers. Since Ras signaling has been reported to be involved in neuronal morphology (see chapter II.3.1), the brain morphology of Rasgrp1 was studied subsequently. Dendrite morphogenesis was analyzed in detail using hippocampal cell culture. Also, possible physiological implications of Rasgrp1 were studied. Field and whole cell recordings on acute hippocampal slices were carried out to investigate basal synaptic transmission and LTP. Next, the study examined the possibility that lack of Rasgrp1 disturbed the architecture of the synaptic network in the hippocampus. A detailed immunohistochemical analysis of excitatory and inhibitory pre- and postsynapses was used to detect any alterations in the synaptic architecture of the CA1 region. A biochemical analysis of hippocampal homogenates and PSD fractions gave insights into the molecular components that are involved in Rasgrp1 function.

Taken together, this study is the first to systematically analyze the neuronal function of Rasgrp1 and thereby to provide valuable information to the understanding Ras signaling in the brain. 


\section{Material}

\section{III.1. Apparatus and Tools}

\begin{tabular}{|l|c|}
\hline \multicolumn{1}{|c|}{ Product } & Source \\
\hline Agarose gel imager & Intas GmbH \\
\hline Amplifier, Multiclamp 700B & Molecular Devices (formally Axon Instruments) \\
\hline Apotome Microscope Axio Imager Z.1 & Zeica Microsystems \\
\hline Binocular MZ16F, Fluorescence & Sorvall \\
\hline Centrifuge, RC6 & Leica Microsystems \\
\hline Confocal laser scanning microscope TCS-SP2 & Reichert-Jung \\
\hline Cryotome & Agfa-Gevaert GmbH \\
\hline Developing machine for ECL films & Olympus Optical CO., (Europa) GmbH \\
\hline Fluorescence Microscope Olympus BX61 & Liebherr, Heraeus über Thermo Electron \\
\hline Freezers & B. Braun Biotech International GmbH \\
\hline Glass-teflon potters & Adolf Kühne AH, Heraeus GmbH \\
\hline Incubators & Molecular devices \\
\hline Microtiter plate reader & BIO-RAD \\
\hline Mini Protean II system & Paul Marienfeld GmbH \\
\hline Neubauer improved cell counting chamber & MJ Research \\
\hline PCR machine PTC-225 & eppendorf \\
\hline PCR-Thermocycler PTC 225 & MJ Research, Bio-Rad Laboratories GmbH \\
\hline Thermomixer compact & IKA Labortechnik \\
\hline Tissue slicer & Beckman \\
\hline Ultra Turrax & Beckman \\
\hline Ultracentrifuge Optima TLX & Branson \\
\hline Ultracentrifuge, L-60 & \\
\hline Ultrasonic waterbath & \\
\hline
\end{tabular}

\section{III.2. Consumables and Kits}

\begin{tabular}{|c|c|c|}
\hline Product & Source & $\begin{array}{c}\text { Catalogue } \\
\text { number }\end{array}$ \\
\hline BCA assay kit & Pierce, Thermo Scientific & $23228 \& 1859078$ \\
\hline Bradford assay kit & BIO-RAD & $500-0006$ \\
\hline coverslips $\varnothing 13 \mathrm{~mm}$ & Menzel Gläser & \\
\hline coverslips $\varnothing 23 \mathrm{~mm}$ & Menzel Gläser & \\
\hline culture plates, 25 well & Greiner bio-one & 662160 \\
\hline culture plates, 6 well & Greiner bio-one & 657160 \\
\hline ECL films & Amersham Biosciences & \\
\hline ECL kit & Amersham Biosciences & RPN2106V1 \& 2 \\
\hline Filter paper Whatman 3MM & Whatman International Ltd. & \\
\hline glass slides $76 \times 26 \mathrm{~mm}$ & Menzel Gläser & \\
\hline Microfilters $(22 \mu \mathrm{m})$ & Millipore & \\
\hline Microtiter plates & Roth & 9293.1 \\
\hline $\begin{array}{l}\text { nexttec }{ }^{\mathrm{TM}} \text { Genomic DNA } \\
\text { Isolation Kit }\end{array}$ & Nexttec & 10.924 \\
\hline $\begin{array}{l}\text { Nitrocellulose Membrane, } 0.2 \\
\mu \mathrm{m}\end{array}$ & Schleicher \& Schuell & \\
\hline PCR plates & ThermoScientific & \\
\hline plastic tubes & eppendorf & \\
\hline T-75 culture flasks & nunc & 157400 \\
\hline Ultracentrifugaion tubes & Beckman & \\
\hline
\end{tabular}


III.3. Software

\begin{tabular}{|l|c|}
\hline \multicolumn{1}{|c|}{ Product } & Source \\
\hline Axiovision R4.1 & ZEISS \\
\hline CellP 2.7 & Olympus Soft Imaging GmbH \\
\hline IGOR Pro 4.03 & WaveMetrics, Inc. \\
\hline ImageJ 1.43k & http://rsb.info.nih.gov/ij/index.html \\
\hline Leica Application Suite (Binocular) & Leica Microsystems \\
\hline Photoshop CS3 & Adobe \\
\hline WinWCP & http://spider.science.strath.ac.uk/sipbs/software_ses.htm \\
\hline
\end{tabular}

\section{III.4. Chemicals, Media and Solutions}

\begin{tabular}{|c|c|c|}
\hline Product & Source & $\begin{array}{c}\text { Catalogue } \\
\text { number }\end{array}$ \\
\hline agarose & Life Technologies (formerly Invitrogen/Gibco) & $15510-27$ \\
\hline ammonium persulfate & Merck & 1.01217 .1000 \\
\hline Bromphenol Blue & Pierce & 20730 \\
\hline $\mathrm{CaCl}_{2} \times 2 \mathrm{H}_{2} \mathrm{O}$ & Merck & 2382 \\
\hline cyclothiazide & Tocris Bioscience & 0713 \\
\hline DAPI & AppliChem & A1001 \\
\hline DMEM & Life Technologies (formerly Invitrogen/Gibco) & $41966-029$ \\
\hline DNA Ladder, $1 \mathrm{~Kb}$ & Life Technologies (formerly Invitrogen/Gibco) & $15615-024$ \\
\hline DTT (dithiotreitol) & BioMol & 04010 \\
\hline EDTA & Merck & 1.08418 .0250 \\
\hline EGTA & Sigma-Aldrich GmbH & E3889 \\
\hline Ethanol & Merck & 1.00983 .1011 \\
\hline Fluoromount-G & Southern Biotech & 0100-01 \\
\hline gabazine & Sigma-Aldrich GmbH & SR 95531 \\
\hline gabazine & Tocris Bioscience & 1262 \\
\hline GlutaMAX'MI & Life Technologies (formerly Invitrogen/Gibco) & $35050-038$ \\
\hline Glycine & Merck & 1.04201 .1000 \\
\hline Goat Serum & Life Technologies (formerly Invitrogen/Gibco) & \\
\hline HBSS & Life Technologies (formerly Invitrogen/Gibco) & $14170-088$ \\
\hline HEPES & Sigma-Aldrich GmbH & H3375 \\
\hline Isofluran & DeltaSelect & \\
\hline $\mathrm{KCl}$ & Merck & 1.04936 .1000 \\
\hline L-AMPA & Tocris Bioscience & \\
\hline Methanol & L.T. Baker & \\
\hline $\mathrm{MgCl}_{2} \times 6 \mathrm{H}_{2} \mathrm{O}$ & Merck & 1.05388 .1000 \\
\hline $\mathrm{Na}_{2} \mathrm{HPO}_{4}$ & Merck & 1.06346 .0500 \\
\hline $\mathrm{NaCl}$ & Merck & 1.6404 .1000 \\
\hline $\mathrm{NaH}_{2} \mathrm{PO}_{4} \times \mathrm{H}_{2} \mathrm{O}$ & Merck & 1.6329 .0500 \\
\hline $\mathrm{NaOH}$ & Merck & 1.06498 .1000 \\
\hline Neurobasal A Medium (NBA) & Life Technologies (formerly Invitrogen/Gibco) & $10888-022$ \\
\hline Papain & Worthington & $36 K 9030$ \\
\hline Paraformaldehyde (PFA) & Serva & 31628 \\
\hline PBS (for cell culture) & PAA & $\mathrm{H} 15-002$ \\
\hline $\begin{array}{l}\text { Penicillin/Streptomycin } \\
10,000 / 10,000 \mathrm{U} / \mathrm{mL}(\mathrm{P} / \mathrm{S})\end{array}$ & Life Technologies (formerly Invitrogen/Gibco) & $15140-122$ \\
\hline PLL & Sigma-Aldrich GmbH & P4707 \\
\hline Ponceau S & Sigma-Aldrich GmbH & P3504 \\
\hline REDTaq $^{\text {TM }}$ DNA Polymerase & Sigma-Aldrich GmbH & D4309 \\
\hline $\begin{array}{l}\text { REDTaq }{ }^{\mathrm{TM}}, 10 x \text { Reaction } \\
\text { Buffer }\end{array}$ & Sigma-Aldrich $\mathrm{GmbH}$ & B5926 \\
\hline SDS (sodium dodecyl-sulfate) & Sigma-Aldrich GmbH & L5750 \\
\hline skim milk & frema & \\
\hline Sucrose & $\begin{array}{c}\text { Merck } \\
\text { Sigma-Aldrich GmbH }\end{array}$ & $\begin{array}{l}1.07651 .1000 \\
\text { s7903pis }\end{array}$ \\
\hline
\end{tabular}


Chemicals, Media and Solutions (continued)

\begin{tabular}{|l|c|c|}
\hline \multicolumn{1}{|c|}{ Product } & Source & \multicolumn{1}{|c|}{$\begin{array}{c}\text { Catalogue } \\
\text { number }\end{array}$} \\
\hline $\begin{array}{l}\text { Temed }\left(\mathrm{N}, \mathrm{N}^{\prime} \mathrm{N}^{\prime}, \mathrm{N}^{\prime}-\right. \\
\text { Tetramethyl-ethylenediamine) }\end{array}$ & Serva & 35925.01 \\
\hline tri-brom-ethanol & Sigma-Aldrich $\mathrm{GmbH}$ & $\mathrm{T} 48402$ \\
\hline Tris Base & Sigma-Aldrich GmbH & $\mathrm{T} 1503$ \\
\hline Triton X-100 & Roche & 10.789704 .100 \\
\hline Trypsin-Inhibitor & Roche Holding GmbH & 1078 \\
\hline TTX (tetrodotoxin) & Tocris Bioscience & P7949 \\
\hline Tween 20 & Sigma-Aldrich GmbH & 49159 \\
\hline$\alpha-\mathrm{D}(+)$ Glucose $\mathrm{H}_{2} \mathrm{O}$ & Fluka & \\
\hline
\end{tabular}

\section{III.5. Antibodies}

\begin{tabular}{|c|c|c|c|c|c|}
\hline $\begin{array}{c}\text { Primary } \\
\text { antibodies }\end{array}$ & Source & $\begin{array}{c}\text { Catalogue } \\
\text { number }\end{array}$ & Immunoblot & $\begin{array}{c}\text { Immuno- } \\
\text { histochem. }\end{array}$ & $\begin{array}{l}\text { Immuno- } \\
\text { cytochem. }\end{array}$ \\
\hline gp-a-VGAT & Synaptic Systems & 131004 & - & $1: 4,000$ & - \\
\hline $\begin{array}{l}\text { ms-a-Map2 clone } \\
\text { AP20 }\end{array}$ & Millipore/Chemicon & MAB3418 & $1: 2000$ & - & - \\
\hline ms-a-NMDAR1 M68 & Synaptic Systems & 114011 & $1: 1,500$ & - & - \\
\hline $\begin{array}{l}\text { ms-a-PSD95 } \\
\text { [6G6-1C9] }\end{array}$ & Abcam & ab2723 & $1: 8,000$ & $1: 2,000$ & - \\
\hline $\begin{array}{l}\text { ms-a-Ras (clone } \\
\text { RAS10), H-,N-,Kras }\end{array}$ & Millipore (Upstate) & $05-516$ & $1: 2,000$ & - & - \\
\hline ms-a-Rasgrp(199) & Santa Cruz & sc-8430 & $1: 500$ & $1: 1,500$ & - \\
\hline $\begin{array}{l}\text { ms-a- } \\
\text { Synaptophysin1 } \\
\text { clone } 7.2\end{array}$ & Synaptic Systems & 101011 & $1: 40,000$ & - & - \\
\hline rb-a-Calbindin D-28K & Millipore Chemicon & AB1778 & - & $1: 5,000$ & - \\
\hline rb-a-Calnexin & $\begin{array}{c}\text { Stressgene/ Assay } \\
\text { Designs }\end{array}$ & SPA-860 & - & $1: 1,000$ & - \\
\hline rb-a-Calretinin & Millipore Chemicon & AB5054 & - & $1: 2,000$ & - \\
\hline rb-a-EEA1 & Abcam & 2900 & - & $1: 500$ & - \\
\hline rb-a-GAD65/67 & Millipore Chemicon & AB1511 & - & $1: 800$ & - \\
\hline rb-a-Giantin & Abcam & ab24586 & - & $1: 2,000$ & - \\
\hline $\begin{array}{l}\text { rb-a-GluR1 (rabbit } \\
\text { monocl.) }\end{array}$ & Millipore Upstate & 04-855 & $1: 800$ & - & - \\
\hline
\end{tabular}




\begin{tabular}{|l|c|c|c|c|c|}
\hline $\begin{array}{c}\text { Primary } \\
\text { antibodies } \\
\text { (continued) }\end{array}$ & Source & $\begin{array}{c}\text { Catalogue } \\
\text { number }\end{array}$ & Immunoblot & $\begin{array}{c}\text { Immuno- } \\
\text { histochem. }\end{array}$ & $\begin{array}{c}\text { Immuno- } \\
\text { cytochem. }\end{array}$ \\
\hline rb-a-GluR2 & Synaptic Systems & 182103 & $1: 3,000$ & - & - \\
\hline rb-a-GluR2/3 & Millipore Chemicon & AB1506 & $1: 800$ & $1: 500$ & $1: 500$ \\
\hline rb-a-Map2 & Millipore Chemicon & AB5622 & - & $1: 500$ & - \\
\hline rb-a-NMDAR2A & Millipore Chemicon & AB1555P & $1: 2,000$ & - & - \\
\hline rb-a-NMDAR2B & Millipore Chemicon & AB1557P & $1: 2,000$ & - & - \\
\hline rb-a-Parvalbumin & Swant & PV-28 & - & $1: 4,000$ & - \\
\hline rb-a-Synapsin1/2 & Synaptic Systems & 106002 & $1: 4,000$ & $1: 1,000$ & - \\
\hline rb-a-VGLUT1 & Synaptic Systems & 135302 & - & \\
\hline
\end{tabular}

\begin{tabular}{|c|c|c|c|c|c|}
\hline $\begin{array}{l}\text { Secondary } \\
\text { antibodies }\end{array}$ & Source & $\begin{array}{c}\text { Catalogue } \\
\text { number }\end{array}$ & $\begin{array}{c}\text { Immuno- } \\
\text { blot }\end{array}$ & $\begin{array}{c}\text { Immuno- } \\
\text { histochem. }\end{array}$ & $\begin{array}{l}\text { Immuno- } \\
\text { cytochem. }\end{array}$ \\
\hline $\begin{array}{l}\text { gt-a-gp Alexa Fluor® } \\
555\end{array}$ & $\begin{array}{c}\text { Life Technologies } \\
\text { (formerly } \\
\text { Invitrogen/Gibco) }\end{array}$ & A-21435 & - & $1: 1,200$ & - \\
\hline $\begin{array}{l}\text { gt-a-ms Alexa Fluor }{ }^{\circledR} \\
488\end{array}$ & $\begin{array}{c}\text { Life Technologies } \\
\text { (formerly } \\
\text { Invitrogen/Gibco) }\end{array}$ & A-11029 & - & $1: 1,200$ & $1: 2,000$ \\
\hline $\begin{array}{l}\text { gt-a-ms Alexa Fluor } \\
555\end{array}$ & $\begin{array}{c}\text { Life Technologies } \\
\text { (formerly } \\
\text { Invitrogen/Gibco) }\end{array}$ & A-21424 & - & $1: 1,200$ & $1: 2,000$ \\
\hline $\begin{array}{l}\text { gt-a-rb Alexa Fluor® } \\
488\end{array}$ & $\begin{array}{c}\text { Life Technologies } \\
\text { (formerly } \\
\text { Invitrogen/Gibco) }\end{array}$ & A-11034 & - & $1: 1,200$ & $1: 2,000$ \\
\hline $\begin{array}{l}\text { gt-a-rb Alexa Fluor® } \\
555\end{array}$ & $\begin{array}{c}\text { Life Technologies } \\
\text { (formerly } \\
\text { Invitrogen/Gibco) }\end{array}$ & A-21429 & - & $1: 1,200$ & $1: 2,000$ \\
\hline gt-a-ms HRP & $\begin{array}{c}\text { Jackson } \\
\text { Immunoresearch }\end{array}$ & $115-035-146$ & $1: 10,000$ & - & - \\
\hline gt-a-rb HRP & $\begin{array}{c}\text { Jackson } \\
\text { Immunoresearch }\end{array}$ & $111-035-144$ & $1: 10,000$ & - & - \\
\hline
\end{tabular}




\section{Methods}

\section{IV.1. Animals}

Animals were hold in the animal house of the MPI of Experimental Medicine under the excellent care of Inga Schauenberg and Astrid Ohle. For comparative analysis of Rasgrp1 KO and WT mice, animals between 2.5 and 5 weeks of age were examined.

\section{IV.1.1. Genotyping}

Genomic DNA was prepared from mouse-tail samples using the Nexttec genomic DNA preparation kit. It contains a "clean" plate, a "DNA plate", a "prep" plate containing columns filled with DNA binding resin, lysis (LG1), "prep-", washing and elution buffers. Tissue samples were lysed in LG1 for 30 min gently shaking (300 $\mathrm{rpm}$, Thermo mixer) at $60{ }^{\circ} \mathrm{C}$. The prep- plate was equilibrated by incubation with "prep" buffer for 5 min. Residual prep- buffer was removed by centrifugation. After that, the tissue lysate was applied onto the columns. During a 1 min incubation time, DNA bound to the resin. The rest of the lysate was removed by centrifugation. Bound DNA was washed with the ethanol-containing washing buffer. The DNA was removed from the columns by adding elution buffer and collected in the DNA plate by centrifugation. Typically, $1 \mu \mathrm{L}$ of this sample was used for the genotyping PCR.

The PCR sample for genotyping was prepared as follows: $2.1 \mu \mathrm{L}$ REDTaq Reaction buffer, $0.8 \mu \mathrm{L}$ dNTPs (10 $\mu \mathrm{M}, 2.5 \mu \mathrm{M}$ each), $15.5 \mu \mathrm{L}$ ds $_{2} \mathrm{O}, 0.08 \mu \mathrm{L}$ oligo $8587(50 \mu \mathrm{M}), 0.08 \mu \mathrm{L}$ oligo $8588(50 \mu \mathrm{M}), 0.16 \mu \mathrm{L}$ oligo $8589(100 \mu \mathrm{M}), 1 \mu \mathrm{L}$ DNA.

\section{Oligos:}

$$
\begin{array}{ll}
\text { \# 8588: 5'-CCTGAGCACATGGGCACATAG -3' } & \text { (Dr. Andrea Betz) } \\
\text { \# 8587: 5'-GCATTCCACCACTGAGCTAGG -3' } & \text { (Dr. Andrea Betz) } \\
\text { \# 8589: 5'-GCTGATGCTTCAGAGCCGAGT -3' } & \text { (Dr. Andrea Betz) }
\end{array}
$$

The reaction was performed using a PCR-Thermocycler PTC 225 (BIO-RAD) and the following protocol:

\begin{tabular}{|l|l|l|l|l|}
\hline & Step & Temp. & Duration & Cycles \\
\hline 1.) & Initial denaturation & $94^{\circ} \mathrm{C}$ & $2{ }^{\prime}$ & - \\
\hline \multirow{3}{*}{2)}$. & Denaturation & $94^{\circ} \mathrm{C}$ & $30^{\prime \prime}$ & \multirow{3}{*}{$35 \mathrm{x}$} \\
\cline { 2 - 4 } & Annealing & $60^{\circ} \mathrm{C}$ & $45^{\prime \prime}$ & \\
\cline { 2 - 5 } & Elongation & $72^{\circ} \mathrm{C}$ & $30^{\prime \prime}$ & \\
\hline \multirow{2}{*}{3)}$. & Final elongation & $72^{\circ} \mathrm{C}$ & $2^{\prime}$ & - \\
\hline 4.$)$ & Store & $10^{\circ} \mathrm{C}$ & $\infty$ & - \\
\hline
\end{tabular}


$15 \mu \mathrm{L}$ of the resulting PCR sample containing amplified DNA were analyzed on a $2 \%$ agarose gel. The WT specific fragment is $314 \mathrm{bp}$ and the KO fragment $378 \mathrm{bp}$ in length.

\section{IV.2. Immunohistochemistry}

\section{Buffer:}

$0.1 \mathrm{MPB}$ was prepared from 19 parts $0.2 \mathrm{M} \mathrm{Na}_{2} \mathrm{HPO}_{4}, 81$ parts $0.2 \mathrm{M} \mathrm{NaH}_{2} \mathrm{PO}_{4}$ and 100 parts $\mathrm{ds}_{2} \mathrm{O}$ (81:19:100)

Avertin solution: $150 \mu \mathrm{L}$ tri-brom-ethanol, $4.5 \mathrm{~mL} 0.9 \% \mathrm{NaCl}, 400 \mu \mathrm{l}$ EtOH

$\mathrm{PO}_{4}$ buffer, $2 \mathrm{L:} 15.4 \mathrm{~g} \mathrm{NaOH}, 67.3 \mathrm{~g} \mathrm{NaH}_{2} \mathrm{PO}_{4} \times \mathrm{H}_{2} \mathrm{O}$

Animals were deeply anesthetized with 300-400 $\mu \mathrm{L}$ Avertin and transcardially perfused with $4 \%$ paraformaldehyde (PFA) in $0.1 \mathrm{M}$ PB for $10 \mathrm{~min}$. Brains were postfixed o/n in $0.1 \mathrm{M}$ PB (2 \% PFA, $2 \%$ glutaraldehyd), rinsed twice in $0.1 \mathrm{M} \mathrm{PB}$, and cryoprotected o/n by incubation in $30 \%$ sucrose in $0.1 \mathrm{M}$ PB. $40 \mu \mathrm{m}$ thick coronal sections were cut using a cryotome, collected in $0.1 \mathrm{M} \mathrm{PB}$ and then stored at $4{ }^{\circ} \mathrm{C}$.

For blocking and permeabilization, sections were rinsed with $0.1 \mathrm{MPB}$ and subjected to $0.1 \mathrm{M}$ PB containing $5 \%$ goat serum (GS) and $0.3 \%$ Triton $\mathrm{X}-100$ for 1 $\mathrm{h}$ at RT. Primary antibodies were diluted in $0.1 \mathrm{M}$ PB containing $2 \%$ GS and $0.3 \%$ Triton X-100. The final dilutions were as stated in chapter III.5. The sections were incubated with the primary antibodies for $36 \mathrm{~h}$ at $4{ }^{\circ} \mathrm{C}$ and slow, bidirectional shaking. Following that, the sections were rinsed three times with $0.1 \mathrm{M}$ PB for $5 \mathrm{~min}$. The secondary antibodies (raised in goat, conjugated to the fluorochromes Alexa555 or Alexa488, 1:1200 final dilution) were prepared in 0.1 M PB containing $5 \%$ GS and $0.3 \%$ Triton $\mathrm{X}-100$ and added to the section. After incubation for $1 \mathrm{~h}$ at RT in the dark, the sections were repeatedly rinsed with $0.1 \mathrm{M} \mathrm{PB}$ and then subjected to 0.5 $\mu \mathrm{g} / \mathrm{mL}$ DAPI in $0.1 \mathrm{M} \mathrm{PB}$ for $5 \mathrm{~min}$ at RT. Section were rinsed again and mounted in Fluoromount-G. 


\section{IV.3. Cell Culture}

\section{IV.3.1. Preparation of Primary Hippocampal Cell Cultures from P0 Mice}

\section{Solutions \& media:}

Papain solution: DMEM, $0.2 \mathrm{mg} / \mathrm{mL}$ Cystein, $10 \mathrm{mM} \mathrm{CaCl}$, $5 \mathrm{mM}$ EDTA, $5 \mathrm{U} / \mathrm{mL}$ Papain, supplied with Carbogen for at least 20 min

stop solution: FBS-Medium, $2.5 \mathrm{mg} / \mathrm{mL} B S A, 2.5 \mathrm{mg} / \mathrm{mL}$ Trypsin inactivator

NBA complete: NBA medium, 1x B27, 2 mM GlutaMAXTMI, $20 \mathrm{U} / \mathrm{ml}^{\text {Penicillin, } 20}$ $\mu \mathrm{g} / \mathrm{ml}$ Streptomycin

FBS medium: DMEM, $10 \%$ FBS, $500 \mu \mathrm{L}$, MITO+ Serum Extender (1 ampulla/5 ml DMEM), $\mathrm{ml} 20 \mathrm{U} / \mathrm{ml}$ Penicillin

Prior to use, all solutions and media had been sterilized using $22 \mu \mathrm{m}$ microfilters.

P0 littermates from heterozygous (Rasgrp1 +/-) parental animals were genotyped as stated in IV.1.1; homozygous WT and Rasgrp1 KO animals decapitated and the hippocampi quickly prepared in ice-cooled HBSS. Tail samples were kept for post-hoc control of genotypes. During genotyping, culture plates were supplied with 1:12 poly-L-lysine (PLL) solution in PBS and set into the cell culture incubator $\left(37^{\circ} \mathrm{C} ; 5 \% \% \mathrm{CO}_{2}\right)$ for $1 \mathrm{~h}$ for coating. After that, the PLL solution was exchanged with HBSS and the plates set back into the incubator.

Per preparation, separate cell cultures were derived from the hippocampi of 1-3 animals per genotype. The hippocampi were transferred into Papain solution and incubated for $1 \mathrm{~h}$ at $37^{\circ} \mathrm{C}$ and gently shaking (600 rpm, Thermo mixer). After digestion by Papain, the solution was exchanged by stop solution to inhibit remaining Papain. In the stop solution, the digested hippocampi were incubated for $15 \mathrm{~min}$ at 37 ${ }^{\circ} \mathrm{C}$ and gently shaking (600 rpm, Thermo mixer). During that time, the HBSS in the culture plates was exchanged with NBA complete. The plates were set back into the cell culture incubator until further use. After incubation and exchanging of the stop solution with prewarmed NBA $\left(37^{\circ} \mathrm{C}\right)$, the digested hippocampi were mechanically triturated. This was achieved by pipetting the tissue up and down using a 10-200 $\mu \mathrm{L}$ pipette tip. Remaining tissue clusters sedimented during a short ( $\sim \mathrm{min})$ incubation period and the supernatant was transferred into a fresh $1.5 \mathrm{~mL}$ plastic tube. The cell 
concentration in this suspension was determined by counting in a Neubauer counting chamber. The appropriate volume of cell suspension was transferred into the wells of the prepared plates.

For continental cultures, cells were seeded in a density of $170 \mathrm{cells} / \mathrm{mm}^{2}$ onto $13 \mathrm{~mm} \varnothing$ coverslips in 24 well plates. Half of the NBA complete medium was exchanged once a week with fresh medium.

Astrocyte island cultures were kindly provided by Anja Galinski. Quickly, astrocytes were prepped from cortices of PO WT mice. Cortices were digested in Trypsin solution for $20 \mathrm{~min}$ at $37^{\circ} \mathrm{C}$ and gently shaking (600 rpm, Thermo mixer). The Trypsin solution was exchanged with stop solution and the digested cortices incubated ( $15 \mathrm{~min}, 37^{\circ} \mathrm{C}, 600 \mathrm{rpm}$, Thermo mixer). The stop solution was exchanged with FBS medium. Subsequently, the cortices were mechanically triturated and sedimented by short incubation. The supernatant was saved and the cell concentration determined using a Neubauer counting chamber. 800,000 cells were seeded into T-75 culture flasks and grown in the cell culture incubator until a confluent cell layer had formed at the bottom of the flask. These cells were harvested by Trypsin treatment and 12.000 cells each were seeded onto glass $23 \mathrm{~mm} \varnothing$ coverslips in 6 well culture plates. These coverslips had been coated with a Collagene/PLL solution by "stamping"; i.e. using a stamp with grid-like notches, resulting in coated microdots $(\sim 0.5 \mathrm{~mm} \varnothing)$ that alone allowed the adherence of cells. These cells were grown in FBS selection medium until islands composed of astrocyte had formed.

For the seeding of neurons onto the astrocytes, the FBS medium was exchanged with NBA complete and 4000 cells added, resulting in a final density of only 10 cells $/ \mathrm{mm}^{2}$.

\section{IV.3.2. Immunocytochemical Stainings}

\section{Buffer: $0.1 M P B$, preparation as described in section IV.2}

Hippocampal cultures were gently washed with PB and fixed for $15 \mathrm{~min}$ at RT in $0.1 \mathrm{M}$ PB containing $4 \%$ PFA and $2.5 \%$ sucrose. Next, the fixed cultures were washed with $0.1 \mathrm{M}$ PB and incubated with $0.1 \mathrm{M}$ PB containing $0.3 \%$ Triton X-100 and $3 \%$ GS for $30 \mathrm{~min}$. This step is required for permeabilization of the cells and blocking of unspecific epitopes. The primary antibody was added to a final dilution in 
0.1 M PB as stated in III.5. The samples were incubated with the primary antibody $\mathrm{o} / \mathrm{n}$ at $4{ }^{\circ} \mathrm{C}$. The next day, the samples were washed, and incubated with the secondary antibody (goat-anti-rabbit conjugated to the fluorochrome Alexa555, $1: 2000)$ for $1 \mathrm{~h}$ at RT. Upon washing, the samples were subjected to DAPI (0.5 $\mu \mathrm{g} / \mathrm{mL}$ ) in $0.1 \mathrm{M} \mathrm{PB}$ for $5 \mathrm{~min}$ at RT. Again, the samples were washed and mounted on glass slides using the fluoro-protectant Fluoromount $\mathrm{G}$. From incubation with the secondary antibody on, all incubations had been performed in the dark to avoid bleaching of the sample.

\section{IV.4. Microscopy and Image Analysis}

Overview images for qualitative analyses were taken at a fluorescence binocular. For detailed qualitative analyses such as of cellular localization, singleplane confocal images of immunolabeled sections were taken on an inverted TCSSP2 confocal laser-scanning microscope (Leica Microsystems), using a 63x objective and a zoom factor of $2 \mathrm{x}$ to $4 \mathrm{x}$. For analysis of dendrite morphology, images were taken at an Olympus fluorescence microscope, using a 20x objective.

Grid-projection images for analysis of synaptic composition were taken at a ZEISS ApoTome Microscope, using a 63x objective. The basic concept behind the ApoTome is the use of an evenly spaced grid in the aperture plane. The grid is inserted into the light path of the microscope and projects a shadow of the grid lines in the objective focal plane. During acquisition, three separate images of the object are sequentially gathered by shifting the grid projection by one-third between each image capture. The resulting image set contains the sum of contributions from the infocus plane, which are shadowed by sharply defined stripes, plus blurred planes (not focused) that do not contain the distinct striping pattern from the grid. By computing these ray images, the application software produces a single sharp image that is free of the blur arising from remote focal planes.

\section{IV.4.1. Analysis of Dendrite Morphology Using Sholl Analysis (Sholl, 1953)}

Images were obtained with the 20x objective and sequential acquisition settings at maximal resolution (1024x1024) of the Olympus fluorescence microscope. Cells were imaged, processed and analyzed blind to the experimental condition. Cells showing obvious signs of toxicity such as dendrite fragmentation, vacuoles in the cell 
body and unusual lamellopodium formations were excluded from the analysis. Neuronal cells obviously belonging to a minor subpopulation of hippocampal cells such as bipolar cells and "hairy cells" (cells with very many thin and short, non bifurcating dendrites) were excluded as well, leaving a collection of images showing separated, healthy appearing neurons with the common morphological feature of having roughly between 3 and 15 primary dendrites.

Each image was a z-series of 7 to 12 images taken at 0.5-1 $\mu \mathrm{m}$ intervals. Each acquisition of such a z-stack was carried out for each color channel separately to reduce bleed-through. Exposure times were kept at the same level throughout the whole experiment. After taking the pictures, the "Extended Focus" algorithm of the CellP software was applied to each z-series. This algorithm extracts the focused area of each image and than "flattens" the z-series of extracted areas, creating a single picture. In this way even dendrites extending from upper or lower levels of the cell body or dendrites crossing each other could be displayed "in focus". Moreover this procedure leads to a markedly reduced background, resulting in pictures where even thin dendrites could clearly be distinguished from their surroundings.

These images were exported as TIFFs and the cells traced in Photoshop using the magic wand tool. Binary images were generated and analyzed using the Image Sholl analysis plug-in (available on the homepage of the Gosh lab, USCD http://biology.ucsd.edu/labs/ghosh/software/).

\section{IV.4.2. Analysis of Synaptic Composition of the CA1 Region}

Coronal brain sections were immunolabeled as described in IV.2. Images were taken blind to the experimental conditions at the ZEISS ApoTome Microscope. The stratum oriens and the stratum pyramidale were imaged together; the stratum radiatum and the stratum lacunosum moleculare were imaged separately. In the latter, this set of four regions is called "image series". Of each coronal section, both hippocampi were imaged. Of each hippocampus, two series of images were taken. One series covered a lateral part, the other one the medial part of the hippocampus. After imaging, the stacks were exported from the AxioVision software into TIFF formats. The regions of interest (ROIs), displaying the respective lamina without an overlap of other laminae, were extracted using ImageJ.

Although utmost attention was paid to homogenous experimental conditions during immunohistochemical stainings, the intensity of the stainings always displayed 
a slight heterogeneity. Moreover, intensity of stainings is always decreasing with tissue depth due to decreasing antibody penetration. Although the standard imaging procedure for comparative analyses requires setting the focus on a Z-plane that lies in a defined distance from the tissue surface, intensities can differ significantly even though this distance is tightly controlled. To circumvent this problem, a stack was taken in each region. This stack was composed of three slices taken at defined Zdepths beneath the tissue surface $(2 \mu \mathrm{m}, 4 \mu \mathrm{m}$ and $6 \mu \mathrm{m})$. After analysis, the data obtained from these slices were binned.

In total, the data of 12 images were binned per lamina per coronal section: Two parts (medial, lateral) per each of the two hippocampi makes four stacks, each comprised of three slices. Therefore the $n$ - numbers given represent the number of sections analyzed.

\section{IV.4.2.1. Development and Evaluation of Image Processing Methods}

For the analysis of synaptic composition in the hippocampus involving a huge set of images, an ImageJ macro frameset for automatized processing and analysis was developed (Figure IV.1). Imaging slices taken in different Z-planes and exhibited heterogeneous intensities posed a problem, since they had to be processed in the same way. The problem was to find a processing method involving automatized and intensity based thresholding that would not extract too much information of one, and leave too much background in the other slice. Also stacks taken in the different laminae of the hippocampus had to be processed by the same method for comparison. Since the labeled proteins are not expressed to the same extent in each lamina, immunostainings showed differences in intensity, posing the same problem for processing as stated above. For reliable comparison of the numbers of inhibitory and excitatory pre- and postsynapses, also the different immunostainings for PSD95, Synapsin1/2, VGLUT and VGAT had to be processed in the same way. For the development of a processing method that could be applied for all slices of a Z-stack, the different regions imaged and the different immunolabelings examined, it seemed difficult to rely on a purely qualitative approach of evaluating the processed slices, i.e. to test different methods and compare original and processed images by eye. The characteristic puncta found in synaptic stainings appear densely packed in immunohistochemical stainings, making it impossible to tell which puncta represents a synapse and which not. Since many samples had to be evaluated, it would have 
been nearly impossible, to apply the same qualitative criteria to all during an evaluation "by eye".

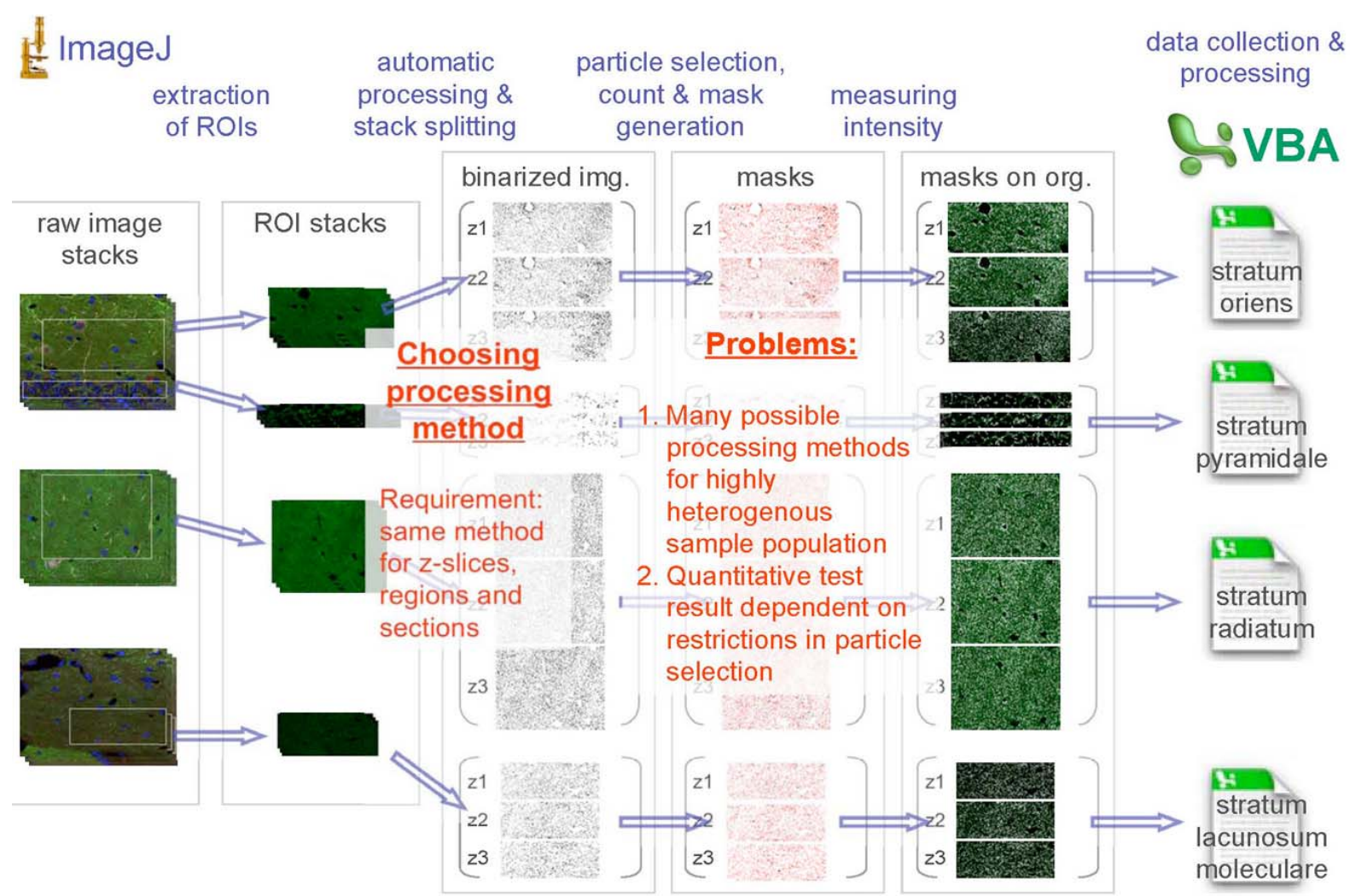

Figure IV.1: Macro frameset for the analysis of synaptic composition

Images for analysis of synaptic composition were processed and analyzed by using a ImageJ macro frameset. The first step was to extract ROIs from the image stacks, followed by stack splitting and automatic processing. Binarized particles were selected by means of area and circularity. Area and number of selected particles were counted and converted to a mask (red). This mask was overlaid onto the original image and intensities measured. The data was exported and collected and processed by Excel VBA macros.

Therefore a method was developed to quantitatively evaluate the processed slices. For testing different processing methods, sections from WT animals were examined (Figure IV.2). These samples comprised of several series (i.e. four stacks showing stratum oriens, pyramidale, radiatum and lacunosum moleculare) taken from different sections of different animals stained in separate experiments, to provide a maximum degree of heterogeneity. The method of choice should meet the following condition: To be able to extract the most homogenous information from this heterogeneous population of samples. As intensity and size of a single synaptic 
punctum reflect the labeling efficiency and distribution of the synaptic protein labeled, the number of puncta is the most general information that could be used for evaluation of homogeneity. In addition to that, the scientific question this experiment was supposed to answer in the first place was, as to whether the number of synapses had changed in the hippocampus of Rasgrp1 KO mice.

Therefore the first constraint of this method was to show the least variance in the numbers of puncta among all the slices that belong to one lamina. To perform test-counts posed another problem, because the selection of puncta to be counted was not exclusively defined by the result of image processing. The resulting particles remaining after processing cannot be counted bona-fide. Some of the particles were composed of aggregating synaptic puncta; some were too small to be taken into account as genuine synapses. Therefore it seemed very helpful to select particles by restrictions for area and circularity. Circularity is a measurement of how spherical an object is and is defined by

$$
\text { circularity }=\frac{2 \sqrt{A}}{P}
$$

$A$ denotes the area and $P$ the perimeter of an object. The resulting values distribute between zero and one. For example, one reflects a perfect sphere and zero reflects a line. However, to select for these measurements imposed additional variables besides the variable of the processing method. These variables are restriction values $(r)$ that denote the minimal and maximal area and circularity. Minimal and maximal restrictions of area and minimal restriction of circularity were tested. The maximal circularity was set to one.

The test counts for the restriction values $(r)$ were performed by counting the particles in the processed images, starting with a minimal $r_{\text {min }}$, e.g. $0.03 \mu \mathrm{m}^{2}$. Then, $r_{\min }$ was increased by a defined value $v\left(\right.$ e.g. $\left.0.01 \mu \mathrm{m}^{2}\right)$ and the puncta were counted again, using $r+v$ (e.g. $\left.0.04 \mu \mathrm{m}^{2}\right)$. This was repeated until $r$ equaled the maximal restriction value $r_{\max }$. The resulting variances in in the particle counts were assessed by the coefficient of variation. The coefficient of variation $\left(c_{v}\right)$ is defined by the ratio of the standard deviation $(\sigma)$ to the mean $(\mu)$ and is used for comparison of variances in populations of samples displaying different mean values:

$$
c_{v}=\frac{\sigma}{\mu}
$$

The $c_{v}$ values were separately assessed for all different laminae. The $c_{v}$ of the corresponding $r$ (e.g. $0.03 \mu \mathrm{m}^{2}$ ) were summarized $\left(c_{v, a l l}\right)$. The $r$ corresponding to the 
lowest $c_{v \text {,all }}$ was chosen for comparison of the processing methods. It was too laborious to test all possible combinations of area and circularity restrictions. Therefore, the restriction values obtained separately were combined for particle analysis in a final, "optimal values" test. It proved, that the variances obtained in this way were very low. Although it is possible, that a combined approach would result in more exact restriction values that could reduce the variance even more, this separate approach was completely sufficient for obtaining very low variances. Of course, this totally depended on the processing method that was evaluated in this way. The processing method chosen for comparative analysis was the one that showed the lowest overall variance in synaptic counts.

\section{\begin{tabular}{|c|} 
Constraint: \\
Method is reliable, if \\
variability in particle count is \\
at its minimum within the test \\
sample population.
\end{tabular}}

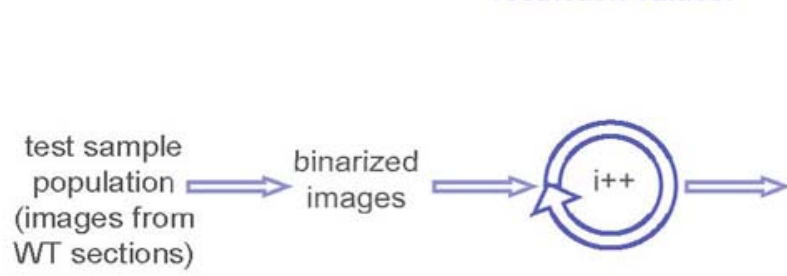

Restricting values:

Presynaptic markers: $\min$ size $=0.09 \mu \mathrm{m}^{2} ; \mathrm{min}$ circularity $=0.5 ; \max$ size $=2.5 \mu \mathrm{m}^{2}$

Postsynaptic marker PSD95: $\min$ size $=0.06 \mu \mathrm{m}^{2}$; min circularity $=0.6 ; \max$ size $=2.5 \mu \mathrm{m}^{2}$
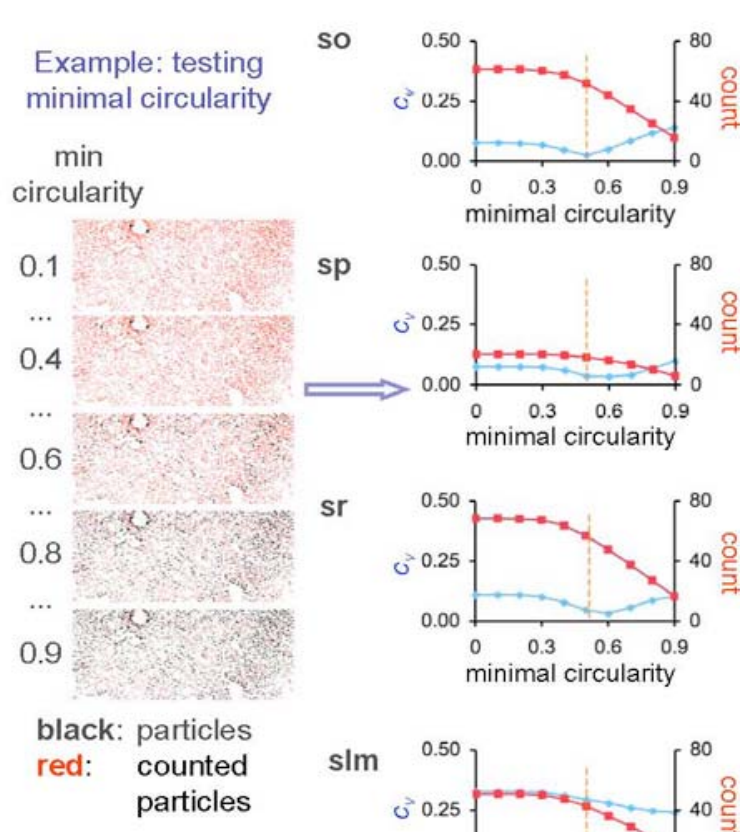

sr
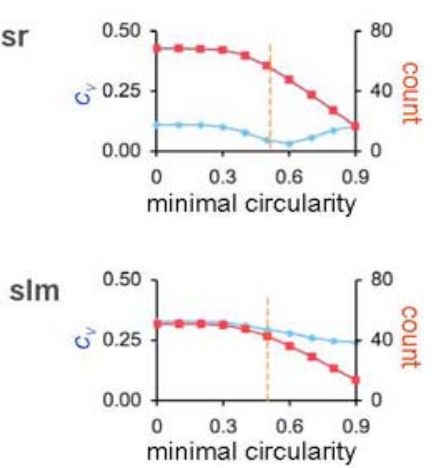

Figure IV.2: Evaluation of the image processing method for analysis of synaptic composition

The processing method was evaluated based on the variability of test counts at different restriction values. Images from WT coronal sections were used as a test samples. The particles in binarized images were automatically selected using different restriction values. The example shows the results of selections at an increasing minimal circularity. The resulting coefficients of variability ( $c_{v}$, left $Y$-axis) and counts (right $Y$-axis) were plotted relative to the minimal circularity. Separate analyses were carried out for each lamina of the hippocampus; so, stratum oriens; 
$\mathrm{sp}$, stratum pyramidale; sr, stratum radiatum; slm, stratum lacunosum moleculare. The orange line represents the minimum of the summarized $c_{v}$.

To realize the processing of a high number of images (i.e. $\sim 480$ per immunolabeling, at an $n$ of 10 sections analyzed), an ImageJ macro was developed for completely automatized processing. Also the iterative analysis of particles at different restriction values was realized by an ImageJ macro. Automatized data analysis was realized by several Excel VBA macros. However, the results of automatized processing and analysis was visually controlled in a subpopulation of randomly selected images (Figure IV.3).

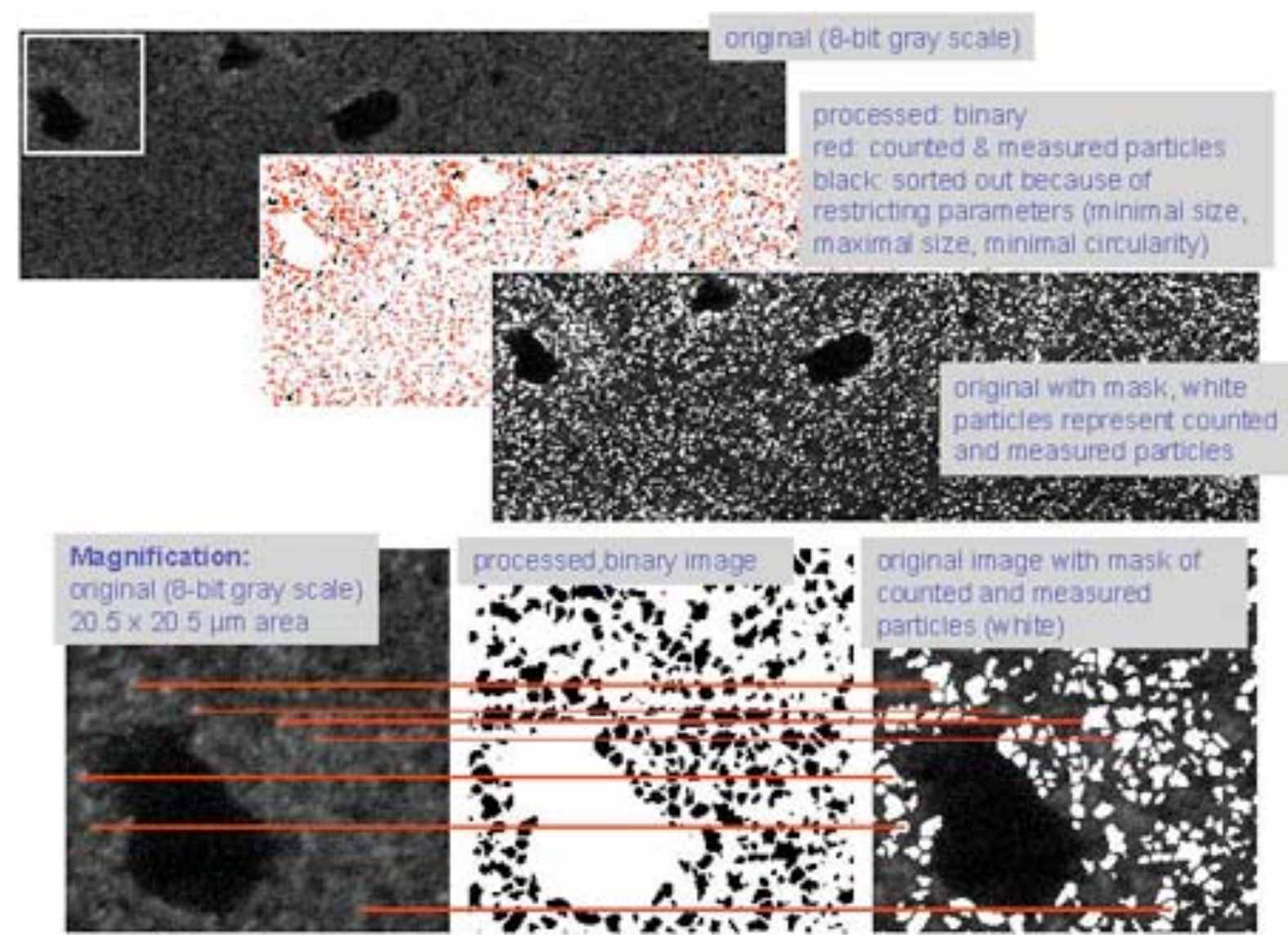

Figure IV.3: Visual control of processing and particle selection

The results of image processing and particle selection were routinely controlled by eye. Both overview and magnification images were assessed. As in the example shown above, the original, the binarized particles and the selected particles were compared. 


\section{IV.4.2.2. The Processing Method of Choice}

The processing method evaluated to provide the best results, was composed of seven sequential steps. The separate processing algorithms are available as ImageJ plug-ins.

(1) Background subtraction, using a rolling ball algorithm with a ball radius of 20 pixels.

(1) Otsu- thresholding, resulting in a binary image.

(2) Watershed segmentation, mask generation, loading mask into original image and deleting unmasked regions.

(3) Gaussian blur with a sigma of 1 .

(4) Background subtraction, using a rolling ball algorithm with a ball radius of 10 pixels.

(5) Isodata thresholding, resulting in a binary image.

(6) Watershed segmentation, mask generation, loading mask into original image for measuring intensity in masked regions.

The rolling ball algorithm can be imagined as a ball rolling over a 3D surface, where the heights are represented as the intensity values of the pixels. The radius determines, which heights are overrun. These heights are designated as background (Sternberg, 1983). However, the radius also determines if the ball can pass a "valley" between two heights. Therefore a lower radius was applied in step (5) to "clean out" theses valleys that could not be subjected to the subtraction of background in step (1).

The thresholding method according to Otsu et al. (1979) takes into account the variances within a distribution of values to distinguish between signal and noise. In case of image thresholding, the variance within the histogram is assessed and the regions showing the highest variances are denoted as background (OTSU, 1979) and removed. Thresholded pixels and signal- pixels were transformed into a binary image.

Watershed segmentation is a way of separating particles that touch. The algorithm calculates a Eucledian distance map, i.e. an image, where the thickest parts of an object receive high values; the parts closer to its boundaries receive low values. Two touching particles can be imagined as two local maxima that are 
connected by a "hanging bridge" of values that from the first maximum first decrease and then increase again towards the second maximum. Watershed segmentation cuts this "hanging bridge" at its minimum, thereby separating the particles. The name watershed is derived from an inversed view of that process. The fattest parts can also be seen as local minima, which are connected by higher values. If water is poured onto this map imagined as a 3D surface, the maxima represent watersheds, which separate the filling pools (Vincent and Soille, 1991).

The binary image was used as a mask for the original picture and the unmasked regions deleted, i.e. denoted as background. Continuing with step (4), the resulting gray-scale image was further processed. Gaussian blur was applied to dilate single background pixels that remained after step (1) - (3) and simultanously to reduce their intensity value. This helped to remove these pixels by subsequent background subtraction using the rolling ball algorithm.

This was followed by isodata thresholding. The isodata algorithm is an iterative procedure for choosing a threshold. The histogram of an image is initially segmented into "background" and "signal" at half of the maximum dynamic range. The mean value of pixels associated with the background and that of those associated with signal are calculated. The average of these two means are used as the new threshold in the next iteration. This procedure is repeated until the threshold does not change anymore (Ridler and Calvard, 1978). In case of the images processed in this study, isodata thresholding resulted in more stringently reduced background as compared to Otsu- thresholding.

The particles in the binary image were selected using the best restriction values as determined in (IV.4.2.1). Subsequently, the areas and number of the selected particles were determined. Then, the selection was converted into a mask and overlaid onto the original image for measurement of intensities.

The areas of particles representing postsynapses labeled for PSD-95 averaged around at $\sim 0.2 \mu \mathrm{m}^{2}$, which would result in a diameter of $500 \mathrm{~nm}$ (if a sphere is assumed, see Figure V.10). Interestingly, this is consistent with the size of the PSD, which was reported to have a mean diameter of $\sim 500 \mathrm{~nm}$ (Feng and Zhang, 2009). The mean area labeled by presynaptic markers ranged from 0.3 to $0.4 \mu \mathrm{m}^{2}$, according to diameters of 620 to $710 \mathrm{~nm}$ (Figure V.11, V.12, V.13). All presynaptic markers used label synaptic vesicles. The synaptic pool fills a great fraction of the presynaptic bouton and depending on the report and image, is $\sim 500-700 \mathrm{~nm}$ in 
diameter (Siksou et al., 2007; Siksou et al., 2009). It is generally larger in diameter than the PSD. This relation as well as the values obtained underlines the reliability of the processing method, whose development has been described here.

\section{IV.5. Electrophysiology on Acute Hippocampal Slices}

Solutions:

S-ACSF (sucrose supplied artificial cerebro-spinal fluid): $87 \mathrm{mM} \mathrm{NaCl}, 26 \mathrm{mM}$ $\mathrm{NaHCO}_{3}, 75 \mathrm{mM}$ sucrose, $25 \mathrm{mM} \mathrm{a-D(+)} \mathrm{Glucose,} 2.5 \mathrm{mM} \mathrm{KCl}, 1.25 \mathrm{mM}$ $\mathrm{NaH}_{2} \mathrm{PO}_{4}, 0.5 \mathrm{mM} \mathrm{CaCl}_{2}, 7 \mathrm{mM} \mathrm{MgCl}{ }_{2}$

Ringer's solution: $119 \mathrm{mM} \mathrm{NaCl}, 26 \mathrm{mM} \mathrm{NaHCO}_{3}, 10 \mathrm{mM} \mathrm{\alpha -D(+)} \mathrm{Glucose,} 2.5 \mathrm{mM}$ $\mathrm{KCl}, 1 \mathrm{mM} \mathrm{NaH} \mathrm{PO}_{4}, 2.5 \mathrm{mM} \mathrm{CaCl}_{2}, 1.3 \mathrm{mM} \mathrm{MgCl}_{2}$, saturated with $95 \%$ $\mathrm{O}_{2}$ and $5 \% \mathrm{CO}_{2}$ (Carbogen) at $\mathrm{pH} 7.4$

Patch-electrode solution: $135 \mathrm{mM}$ KGluc, $20 \mathrm{mM} \mathrm{KCl,} 10 \mathrm{mM}$ HEPES, $0.2 \mathrm{mM}$ EGTA, 2 mM MgATP, pH 7.2

\section{IV.5.1. Preparation of Acute Hippocampal Slices}

For preparation of acute hippocampal slices, animals were anesthetized by laminar flow using evaporated Isofluoran. Brains were quickly, but carefully removed from the skulls and kept covered in icy, Carbogen supplied S-ACSF slush for 5 min. Brain hemispheres were separated and glued onto the cutting platform of a tissue slicer and submerged in S-ACSF slush. $300 \mu \mathrm{m}$ thick horizontal hippocampal slices were prepared at a cutting speed of $0.1 \mathrm{~mm} / \mathrm{s}$ and at an horizontal cutting blade amplitude of $1 \mathrm{~mm}$. After preparation, slices for patch clamp recordings were kept in Carbogen supplied Ringer's solution at $37^{\circ} \mathrm{C}$ for $30 \mathrm{~min}$. After that, slices in Ringer's solution were maintained at RT. Slices for field recordings were transferred directly to Ringers's solution at RT after cutting.

For recordings, slices were transferred onto Lysine-coated glass coverslips. When they had attached at the coverslips, slices where quickly transferred into the recording chamber of the set-up. Recording chambers were perfused by Carbogen saturated Ringer's solution. The perfusion speed was tightly controlled and set to 4-5 $\mathrm{mL} / \mathrm{min}$ prior to each experiment to omit heterogeneous experimental conditions, e.g. different wash-in times for the application of L-AMPA.

Slices for field recordings were kept for maximal 7 hours, slices for patch clamp recordings for maximal 4 hours. 


\section{IV.5.2. Field and Patch Clamp Recordings}

Field EPSP (fEPSP) recordings were performed with low resistance patch clamp electrodes filled with Ringer's solution. The recording electrode was placed in the stratum radiatum area CA1. Schaffer collaterals were stimulated with patch clamp electrodes filled with Ringers's solution at a frequency of $0.05 \mathrm{~Hz}$. Electrophyiolgical signals were recorded with a MultiClamp 700B amplifier (Molecular Devices), lowpass filtered at $1-3 \mathrm{kHz}$ and digitized with $5-10 \mathrm{kHz}$ (AD board, National Instruments) and data were stored on the PC for later off-line analyses. All in-experiment analyses for monitoring as well as post-hoc analyses of field recordings were done in IGOR Pro software. For input-output experiments, the strength of the input signal was determined by measuring the fiber volley. The resulting fEPSPs were recorded. Paired pulse facilitation was examined by monitoring the ratio of second to first fEPSP. LTP was induced by four $100 \mathrm{~Hz}$ stimuli, each $20 \mathrm{~s}$ apart. The resulting potentiation was analyzed as stated in Figure V.8 B.

Single cell voltage clamp recordings were performed in whole cell mode of the patch-clamp technique. Patch clamp electrodes (resistance 2-5 M $\Omega$ ) were filled with patch-electrode solution. Series resistance ranged from $6-25 \mathrm{M} \Omega$ and was continuously monitored throughout the experiment. All patch-clamp experiments were performed in the presence of the GABA receptor-antagonist gabazine $(1 \mu \mathrm{M}), 1$ $\mu \mathrm{M}$ tetrodotoxin (TTX, $1 \mu \mathrm{M})$, and cyclothiazide $(100 \mu \mathrm{M})$. mEPSCs were analyzed starting from 2 min after stable access to the cell had been established. Recordings were automatically analyzed using the WinWCP software. The results of these analyses were visually controlled to remove test pulse artifacts and multiple overlapping mEPSCs. AMPAR currents were evoked by application of $25 \mathrm{nM}$ LAMPA for $15 \mathrm{~min}$. The change in holding current ( $\left.\mathrm{I}_{\mathrm{AMPA}}\right)$ was recorded and analyzed in IGOR Pro software. All raw data was exported to Excel for the design of graphs and diagrams. 


\section{IV.6. Biochemistry \\ IV.6.1. SDS-PAGE and Western Blotting \\ Buffers:}

Seperating gel buffer: $0,5 \mathrm{M}$ Tris- $\mathrm{HCl}(\mathrm{pH} \mathrm{6,8),} \mathrm{0,4 \%} \mathrm{SDS}$

Stacking gel buffer: $1,5 \mathrm{M}$ Tris- $\mathrm{HCl}(\mathrm{pH} 8,8), 0,4 \% \mathrm{SDS}, \sim 0.01 \mathrm{~g}$ Bromphenol Blue

Transfer buffer, 1 L: $3 \mathrm{~g}$ Tris-Base, $14.4 \mathrm{~g}$ Glycine, $200 \mathrm{~mL}$ Methanol

Laemmli Buffer 3x: 140 mM Tris- $\mathrm{HCl}$ ( $\mathrm{pH}$ 6.8), 3 mM EDTA, $39 \%$ sucrose, $10 \%$ SDS, $0.1 \%$ Bromphenol Blue, 150 mM DTT

Ponceau solution: $0.1 \%$ Ponceau S, $5 \%$ acetic acid

Protein samples were separated by sodium dodecyl sulfate polyacrylamide gel electrophoresis (SDS-PAGE). Separating gels contained 10-15\% bis-acrylamide in separating gel buffer. Stacking gels were composed of $3.75 \%$ bis-acrylamide in the stacking gel buffer. The polymerization of the gels was initiated by adding Temed and ammonium-persulfate. Gels were loaded with $8-12 \mu \mathrm{g}$ of protein in Laemmli buffer and run at RT and $25 \mathrm{~mA}$ in BIO-RAD chambers (Mini Protean II system).

Western blots were prepared by the wet transfer method (BIO-RAD MiniProtean II system). Proteins were blotted onto nitrocellulose membrane in transfer buffer. Blotting was performed at $40 \mathrm{~mA}$ and $4{ }^{\circ} \mathrm{C}$ for $16 \mathrm{~h}$. The success of transferring proteins onto the membrane was controlled by Ponceau staining.

\section{IV.6.2. Immunolabeling of Blots}

\section{Buffers:}

TBS (Tris-buffered saline): $20 \mathrm{mM}$ Tris-HCl, $140 \mathrm{mM} \mathrm{NaCl,} \mathrm{pH} 7.5$

TBST (Tris-buffered saline containing Tween 20): TBS, $0.1 \%$ Tween 20

For immunolabelings, blots were blocked in TBST containing $5 \%(\mathrm{w} / \mathrm{v})$ skim milk $5 \%(\mathrm{v} / \mathrm{v})$ and goat serum (GS) for $30 \mathrm{~min}$ at RT. The primary antibody was added to a final concentration as stated in (III.5) and the blots incubated for $1 \mathrm{~h}$ at RT. Goat antibodies reactive to mouse or rabbit and conjugated to horseraddish peroxidase (HRP) were used as secondary antibodies. After removing the primary 
antibody by washing with TBST (5\% skim milk), the secondary antibody was applied in a final dilution of 1:10000 in TBST (5\% skim milk). Secondary antibody and blot were incubated for $1 \mathrm{~h}$ at RT. After that, the blot was washed several times in TBST (5\% skim milk), followed by three washing steps using TBST (without skim milk), and rinsing with TBS.

An ECL signal was evoked by application of the ECL (enhanced chemiluminescence) reagents provided in the ECL detection kit of Amersham Biosciences. Detection of the resulting ECL signal was achieved using ECL sensitive films and subsequent development in a developing machine.

\section{IV.6.3. Subcellular Fractionation}

\section{Buffers:}

sucrose buffer: 4 mM HEPES, 320 mM sucrose, $1 x$ protein inhibitors, $p H 7.3$

\section{$0.8 \mathrm{M}$ sucrose buffer: $4 \mathrm{mM}$ HEPES, $0.8 \mathrm{M}$ sucrose, $1 x$ protein inhibitors}

\section{2 $M$ sucrose buffer: 4 mM HEPES, $1.2 M$ sucrose, $1 x$ protein inhibitors}

Iysis buffer: 4 mM HEPES, $1 x$ protein inhibitors

The brains of three WT mice were dissected on ice. The cerebellum and the brain stem were cut off. Then, the hemispheres were separated and the midbrains removed. All following steps and centrifugations were performed at $4{ }^{\circ} \mathrm{C}$ (cold room) or on ice. The forebrains were pooled in $8 \mathrm{~mL}$ of sucrose buffer in a glass tube and homogenized by 12 up/downs of a teflon homogenizer rotating at $900 \mathrm{rpm}$. Of each intermediate or final sample, a fraction was saved for later analysis. The homogenate was centrifuged at $1,000 \mathrm{~g}$ (SS34 rotor, Sorvall RC6 Centrifuge) for $10 \mathrm{~min}$. The supernatant (S1) was saved and centrifuged at 12,000 g (SS34 rotor, Sorvall RC6 Centrifuge). The pellet (P1) was resuspended in $6 \mathrm{~mL}$ of sucrose buffer and stored for later analysis. After centrifugation of $\mathrm{S} 1$, the part of the supernatant that could clearly be distinguished from heavier fractions was saved $\left(\mathrm{S}_{1}\right)$ and the rest of the $\mathrm{S} 1$ sample washed by supplying $6 \mathrm{~mL}$ of sucrose buffer and centrifuged again $(12,000 \mathrm{~g}$, SS34 rotor, Sorvall RC6 Centrifuge). The resulting supernatant $\left(\mathrm{S}_{2}\right)$ was collected and combined with $S 2{ }_{1}$. The resulting $S 2$ sample was centrifuged at 50,000 rpm (223,160 rpm, Beckman L-60 centrifuge, Type 75Ti fixed angle rotor) for $2 \mathrm{~h}$. The 
resulting final fractions were S3 and P3. P3 was resuspended in $300 \mu \mathrm{L}$ sucrose buffer and stored for analysis, S3 was concentrated using Centricon 10 columns prior to storage, resulting in $\sim 1.5 \mathrm{~mL}$ of sample.

The pellet that had formed in the previous centrifugation $\left(P 2_{1}\right)$ consisted of a lighter and a heavier fraction (P2M). The lighter fraction (P2) was carefully removed and resuspended in $500 \mu \mathrm{L}$ sucrose buffer, transferred into glass tubes, supplemented with $9 \mathrm{~mL}$ lysis buffer and further homogenized by 10 strokes at 1500 rpm of a Teflon homogenizer. The P2M fraction was resuspended in $500 \mu \mathrm{L}$ sucrose buffer and stored for analysis. The lysed P2 fraction was centrifuged at $25,000 \mathrm{~g}$ for 20 min (SS34 rotor, Sorvall RC6 Centrifuge). This resulted in the LP1 and the LS1 fractions. The LS1 fraction was collected and centrifuged at 50,000 rpm for $2 \mathrm{~h}$ (223,160 rpm, Beckman L-60 centrifuge, Type 75Ti fixed angle rotor). The resulting LP2 fraction was resuspended in $150 \mu \mathrm{L}$ sucrose buffer and stored for analysis, the LS2 fraction was concentrated in Centricon 10 columns, resulting in $\sim 300 \mu \mathrm{L}$ sample, which was stored for analysis. The LP1 fraction was resuspended in $1 \mathrm{~mL}$ of sucrose buffer and applied onto a sucrose gradient composed of $5 \mathrm{~mL} 0.8 \mathrm{~mL}$ sucrose buffer on the top of $5 \mathrm{~mL} 1.2 \mathrm{M}$ sucrose buffer in a glass tube. The sample was centrifuged at 19,000 rpm (64206 rpm, Beckman L-60 centrifuge, SW41Ti swing out rotor) for $2 \mathrm{~h}$. The upper interphase (LP1A) the lower interphase (LP1B) and the pellet (LP1C) were collected and stored.

Protein titration of the fractions was achieved by the Bradford method and the concentration adjusted to $1 \mathrm{mg} / \mathrm{mL}$.

\section{IV.6.4. Purification of PSD Fractions}

\section{Buffers:}

homogenization buffer: $320 \mathrm{mM}$ Sucrose, $10 \mathrm{mM}$ HEPES-KOH (pH 7.4), 5 mM EDTA (pH 7.4), $1 x$ protein inhibitors

resuspensiton buffer: $20 \mathrm{mM}$ Tris- $\mathrm{HCl}$ ( $\mathrm{pH}$ 7.4), 2 mM EGTA ( $\mathrm{pH}$ 7.4), $1 \times$ protein inhibitors

Hippocampi were prepared, quickly frozen in liquid nitrogen and stored until enough tissue samples were available for an experiment. In one experiment, PSD fractions were separately prepared from eight to twelve hippocampi. Analyses contained samples from three separate preparations. All steps of the protocol were 
performed at $4{ }^{\circ} \mathrm{C}$ or on ice. Frozen hippocampi were transferred into homogenization buffer in a glass tube and homogenized with 10 gentle up/downs at $900 \mathrm{rpm}$ of a Teflon potter. A fraction of the crude homogenate was saved for later analysis, the rest was centrifuged at $1000 \mathrm{~g}$. The supernatant (S1) was saved and centrifuged at $15000 \mathrm{~g}$. The supernatant (S2) was discarded and the remaining pellet (P2) resuspended in $50 \mu \mathrm{L}$ of resuspension buffer. The protein content was assessed by the Bradford assay and adjusted to $2 \mathrm{mg} / \mathrm{mL}$ by adding Triton $\mathrm{X}-100$ to a final concentration of $1 \%$. Then, the resuspended P2 sample was sonicated in an ultrasonic waterbath for 3 times for $30 \mathrm{~s}$ at $70 \%$ power. Following that, the proteins were solubilized by slow overhead-rotation for $1 \mathrm{~h}$. Then, the protein extracts were collected in ultra-centrifugation tubes and centrifuged at $100000 \mathrm{~g}$ for $1 \mathrm{~h}$. The pellet (PSD sample) was saved and resuspended in resuspension buffer containing $1 \%$ SDS. Again, the sample was agitated by slow rotation for $1 \mathrm{~h}$. Protein levels in homogenate and PSD fraction samples were assessed using the BCA method and samples were adjusted to a final concentration of $1 \mathrm{mg} / \mathrm{ml}$ in resuspension buffer containing 1x Laemmli buffer. Protein samples were loaded on SDS-PAGE gels (10 $\mu \mathrm{g}$ per well), and seperated as described in IV.6.1. Immunolabeling and detection were performed as described in IV.6.2.

Protein levels on the blots were assessed by densitometry using the ImageJ „Analyze Gels“ plug-in. Synaptophysin was used as the reference protein. Protein levels of marker protein were expressed as the ratio of marker to reference protein.

\section{IV.7. Statistics}

If not otherwise stated, all means are represented \pm sem. Also if not otherwise stated, all statistical analyses were performed using the Student's $t$-test. 


\section{Results}

\section{V.1. Distribution of Rasgrp1 in the Mouse Hippocampus}

\section{V.1.1. Strongest Expression of Rasgrp1 is Found in the Stratum Pyramidale}

Previous immunohistochemical studies in rat brain sections have reported strong expression of Rasgrp1 in the hippocampus (Pierret et al., 2001). Immunostainings for light microscopy already indicated an enrichment of Rasgrp1 in somata and apical dendrites of pyramidal cells in hippocampal CA1 and CA3 regions.

I confirmed these results by fluorescence immunostaining for Rasgrp1 and Map2 in mouse coronal sections. Map2 is a well-known marker for dendrites (Bernhardt and Matus, 1984). In the hippocampal CA1 region, Rasgrp1 immunofluorescence was primarily detected in the stratum pyramidale, where it localized to the perinuclear region of cells, but not to the nucleus (Figure V.1 A). Map2 was found almost exclusively in the stratum radiatum and the stratum lacunosum moleculare (Figure V.1 A). These laminae contain the apical dendrites of the pyramidal neurons. High magnification images revealed additional Rasgrp1 immunofluorescence in the proximal region of the stratum radiatum and colocalization with Map2 immunofluorescence, indicating a localization of Rasgrp1 in proximal apical dendrites (Figure V.1 B). Moreover, a faint disheveled staining was detected that could not be attributed to a specific compartment. Overall, these results confirm the findings of Pierret et al. (2001). However, they also clearly show a preferential localization of Rasgrp1 to somata, which could not be concluded from the earlier studies (Pierret et al., 2001). 


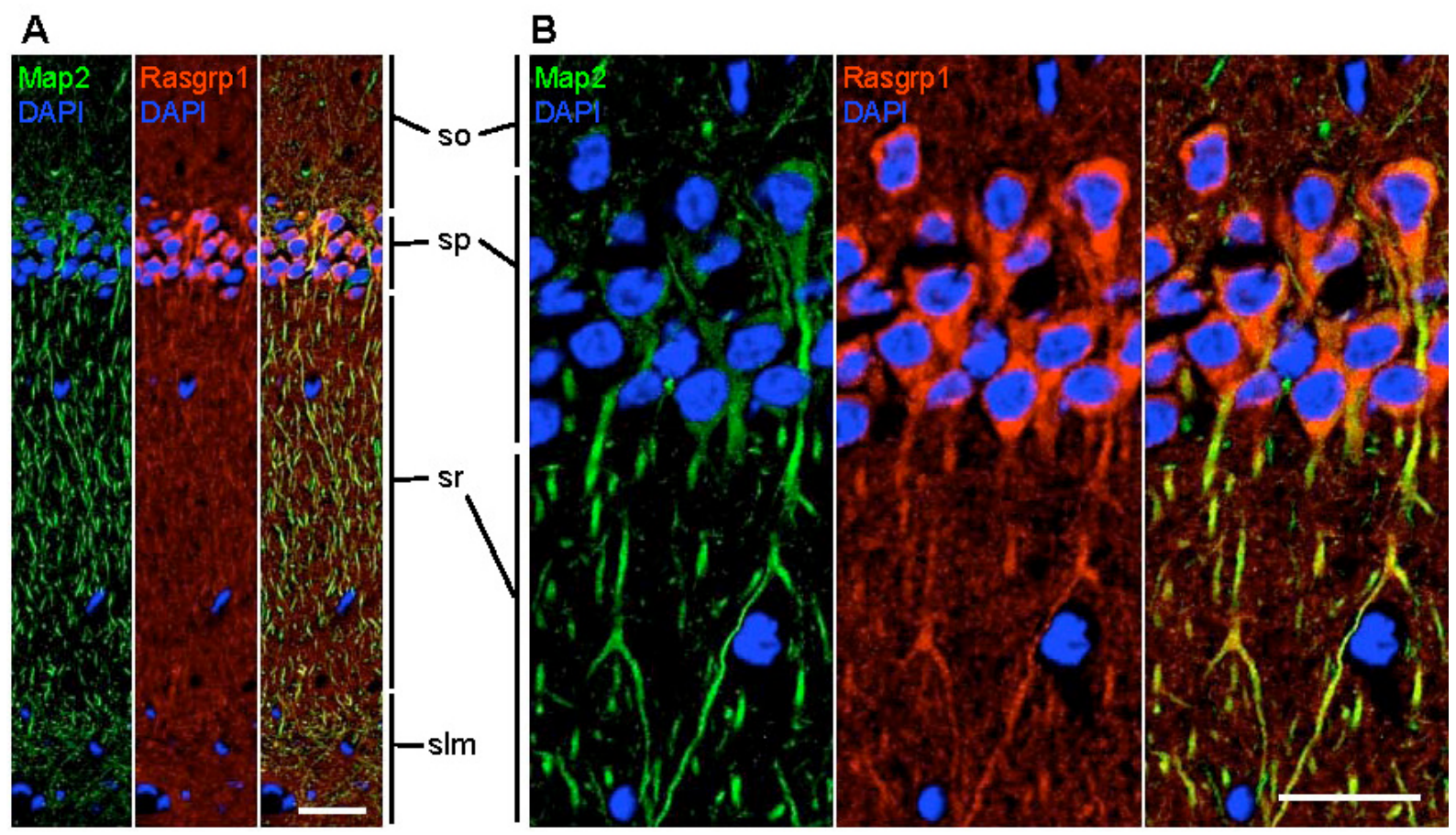

Figure V.1: Regional distribution of Rasgrp1 in the CA1 region of the hippocampus

DAPI staining and immunohistochemical stainings for Rasgrp1 and Map2 were performed to study the distribution of Rasgrp1 in the layers of the CA1 region of the hippocampus.

(A) Strongest immunolabelings of Rasgrp1 was detected in the stratum pyramidale. A disheveled staining of low intensity was detected throughout all strata. Scale bar = $40 \mu \mathrm{m}$. (B) Rasgrp1 was also present in proximal apical dendrites, as indicated by colocalization with Map2. Scale bar $=20 \mu \mathrm{m}$.

so, stratum oriens; sp, stratum pyramidale; sr, stratum radiatum; slm, stratum lacunosum moleculare.

\section{V.1.2. Rasgrp1 is Not Expressed in Inhibitory Neurons}

When observing Rasgrp1 stainings, it became apparent that Rasgrp1 is not present in every cell. This was indicated by costainings of nuclei by DAPI (2-(4amidinophenyl)- $1 \mathrm{H}$-indole-6-carboxamidine). The majority of stained nuclei were surrounded by characteristic Rasgrp1 immunofluorescence, but some were not (Figure V.1 A, B). The stratum pyramidale is named after the prevalent cell type found within it, i.e. the pyramidal cells. A minor fraction of somata found in the stratum pyramidale belongs to inhibitory neurons. Therefore the few cells negative for 
Rasgrp1 immunofluorescence might represent inhibitory neurons. This was tested using markers for inhibitory neurons in immunofluorescence staining experiments.

Parvalbumin is a soluble Calcium binding protein located in the cytoplasm of inhibitory neurons (Mátyás et al., 2004). No expression of Rasgrp1 was found in Parvalbumin-positive somata (Figure V.2 A). Calretinin is another Calcium binding protein localized to a different subpopulation of inhibitory neurons (Mátyás et al., 2004). Also for this marker, no colocalization with Rasgrp1 immunofluorescence was detected (Figure V.2 B). Glutamic acid decarboxylase (GAD) exists in two isoforms (GAD65, officially known as Gad2 and GAD67, officially known as Gad1) that catalyze the decarboxylation of Glutamate to GABA in inhibitory neurons (Erlander et al., 1991). Both Gad isoforms are found at synapses and within somata of inhibitory neurons (Esclapez et al., 1994). GAD65/67 positive somata were negative for Rasgrp1 immunoreactivity (Figure V.2 C). Calbindin is a Calcium binding protein found in a subpopulation of inhibitory neurons as well as in a subset of pyramidal neurons (Mátyás et al., 2004). Prevalent Calbindin immunofluorescence was detected in the layer of somata that lie adjacent to the stratum radiatum, indicating that most of them belong to pyramidal cells (Figure V.2 D). These somata also showed expression of Rasgrp1, whereas other Calbindin positive somata were negative for Rasgrp1. The more dorsally located layers of somata were completely devoid of Calbindin immunofluorescence, but showed Rasgrp1 expression (Figure V.2 D). These results provide strong evidence for an absence of Rasgrp1 in inhibitory neurons and indicate an exclusive expression of Rasgrp1 in pyramidal cells. 
A
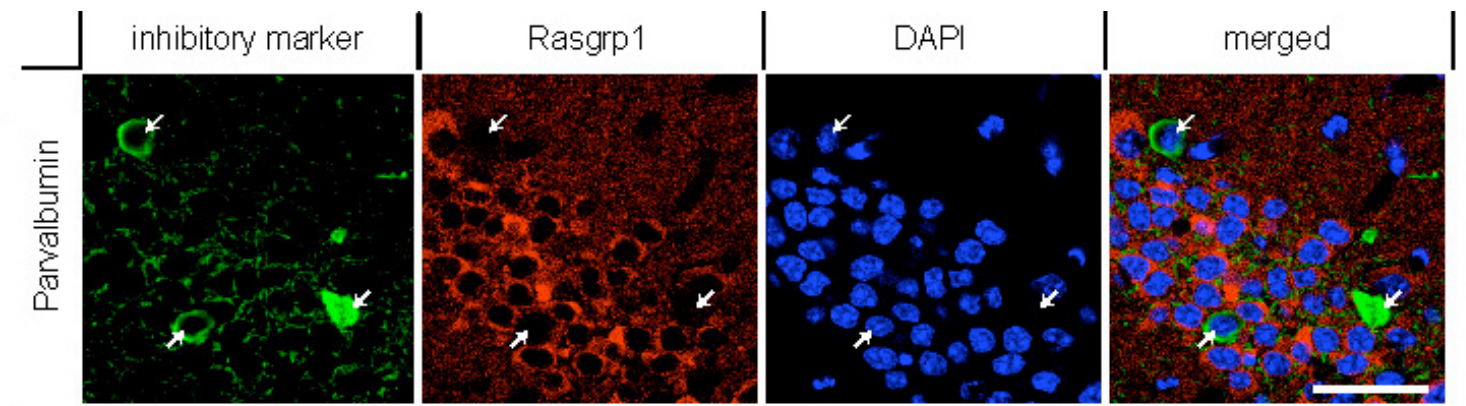

B
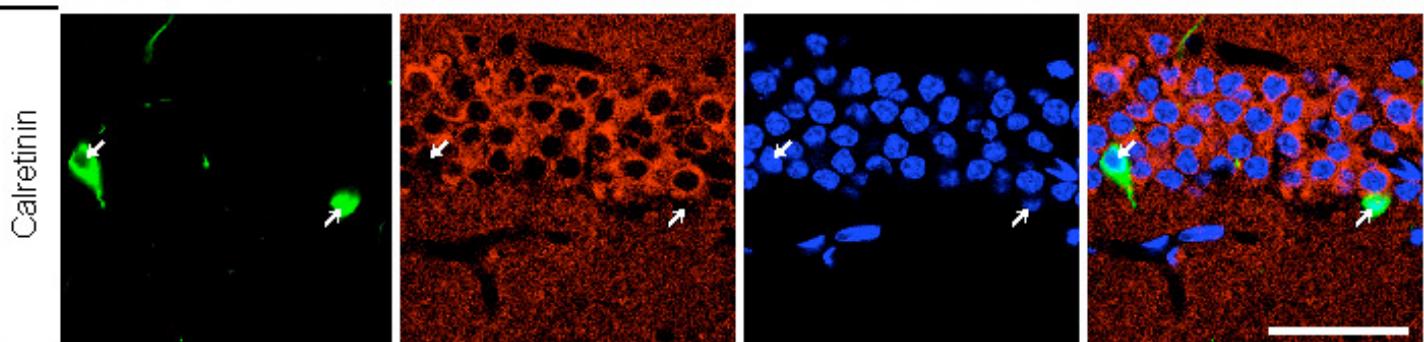

C
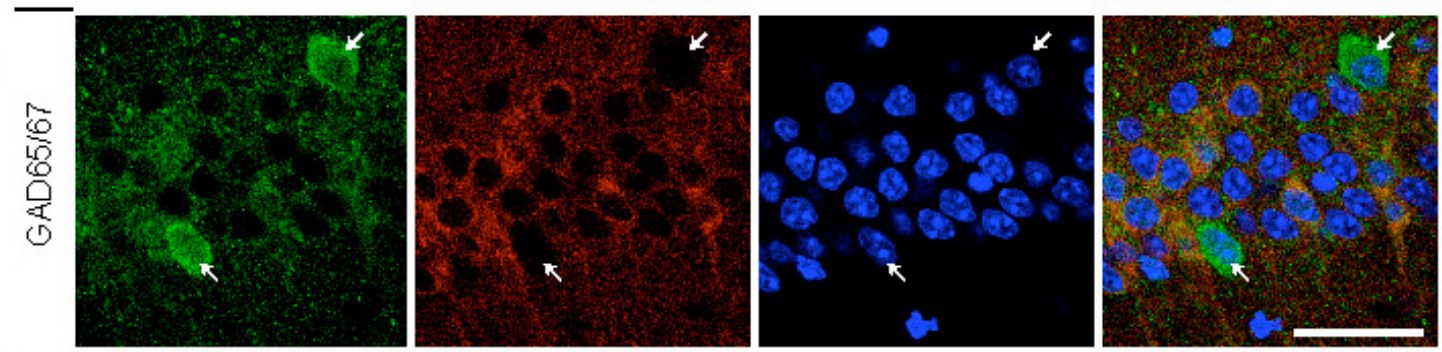

D
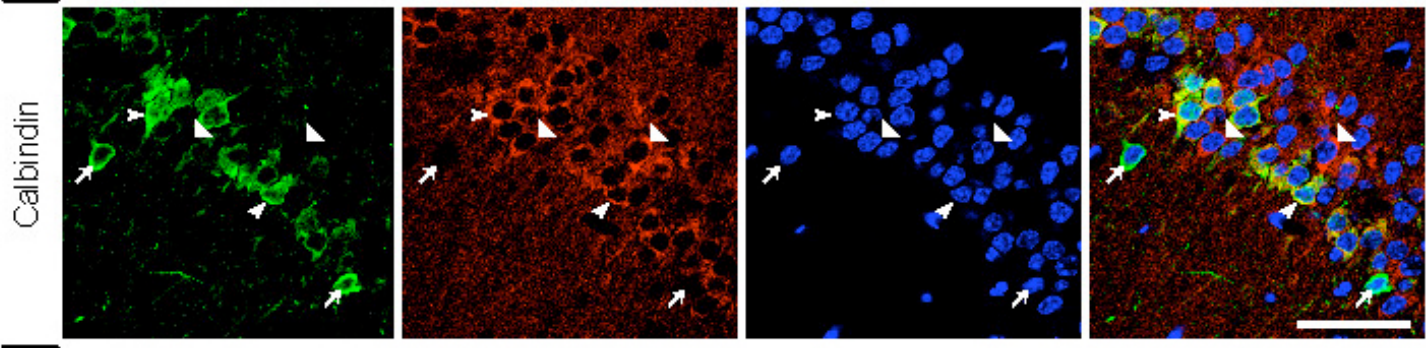

Figure V.2: Cellular distribution of Rasgrp1 in the stratum pyramidale

DAPI staining and immunohistochemical stainings for Rasgrp1 and inhibitory neuron markers showed that Rasgrp1 is not expressed in inhibitory neurons.

(A-C) All somata that expressed Parvalbumin, Calretinin or GAD65/67 were negative for Rasgrp1, as indicated by the arrows. Scale bars $=30 \mu \mathrm{m}(A), 40 \mu \mathrm{m}(B), 30 \mu \mathrm{m}$ (C). (D) Calbindin is expressed in inhibitory neurons and in a subset of pyramidal neurons. Arrows indicate inhibitory neurons that expressed Calbindin but not Rasgrp1. Thin arrowheads indicate pyramidal neurons that are positive for both Rasgrp1 and Calbindin immunoreactivity, and broad arrowheads indicate neurons that only expressed Rasgrp1. Scale bar $=40 \mu \mathrm{m}$. 


\section{V.1.3. Rasgrp1 Preferentially Localizes to the Golgi Apparatus of Neurons}

In many cell lines, Rasgrp1 has been shown to localize to the Golgi apparatus. Also in the neuronal PC12 cell line this specific pattern of localization was observed (Bivona et al., 2003).

To corroborate these findings in neurons, immunolabelings for Rasgrp1 and markers for subcellular compartments were performed. Indeed, Rasgrp1 showed a prominent colocalization with the Golgi apparatus marker Giantin (Figure V.3 A), although Rasgrp1 staining appeared a little more diffuse. In contrast, none or unspecific overlaps of Rasgrp1 staining were apparent when compared to stainings of the ER marker Calnexin (Figure V.3 B), the early endosome maker EEA1 (Figure V.3 C), or the postsynaptic marker GluR2/3 (Figure V.3 D).

Taken together, the above results show that Rasgrp1 is expressed exclusively in pyramidal cells of the hippocampus, and therein specifically localizes to the Golgi apparatus, but not to ER, early endosomes, or postsynapses. 


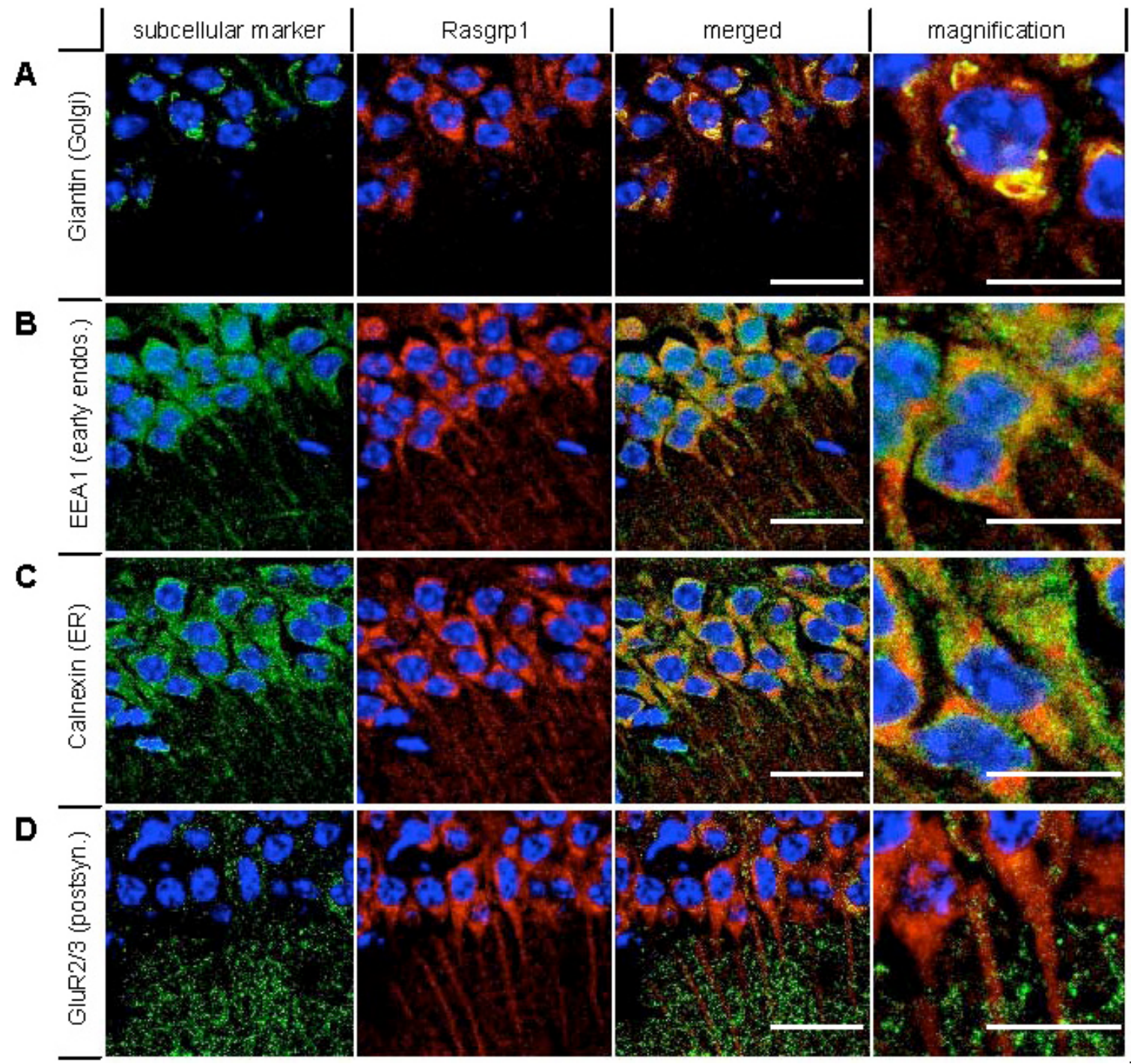

Figure V.3: Subcellular localization of Rasgrp1 in pyramidal neurons

Immunohistochemical stainings for Rasgrp1 and subcellular compartment markers showed that Rasgrp1 localizes to the Golgi apparatus.

(A) Rasgrp1 colocalizes with the Golgi marker Giantin. (B-C) None or unspecific colocalization was observed for markers of early endosomes (EEA1), endoplasmatic reticulum (ER; Calnexin) or postsynapses (GluR2/3). Scale bars columns 1-3 $=20$ $\mu \mathrm{m}$; Scale bars column $4=10 \mu \mathrm{m}$. 


\section{V.1.4. Enrichment of Rasgrp1 in the Microsomal Fraction of Fore Brain}

To further characterize the localization of Rasgrp1, forebrain homogenate and biochemically purified subcellular fractions were blotted, immunolabeled and analyzed for enrichment or depletion of Rasgrp1.

As compared to the homogenate, the fractions enriched in Rasgrp1 were the P3, the S3, the LP1B and the LP1A fraction (in order of band intensity). The fractions depleted in Rasgrp1 were the P1, the P2M, the LP1C, the LP2 and the LS2 fraction (Figure V.4 A). The fraction predominantly enriched in postsynaptic markers such as PSD-95 and the AMPAR subunits GluR2 and GluR3 (Figure V.4 B, C) is the LP1B fraction. It also contains proteins found at the presynaptic membrane (Figure V.4 E, $\mathrm{F}$ ), indicating that it is a fraction composed of both post- and presynaptic membrane. The soluble S3 fraction is enriched in Map2 (Figure V.4 D). Map2 associates to the cytoskeleton and is solubilized when cell structure is destroyed (Matus, 1994). The LP2 fraction was reported to contain synaptic vesicle proteins (Huttner et al., 1983). Presence of the synaptic vesicle proteins Synaptophysin (Syp) and Synapsin (Synapsin1/2; Syn1/2) confirmed, that the LP2 fraction contains these organelles (Figure V.4 E, F). As expected, an antibody recognizing all three classical Ras G proteins revealed their presence in all membranous fractions (Figure V.4 G). Except for the S3 fraction, classical Ras $G$ proteins are found in the same fractions as Rasgrp1. The lack of classical Ras $G$ proteins in the $S 3$ fraction confirms that Ras activation occurs at membranes (Augsten et al., 2006; Bivona et al., 2003). Rasgrp1 found among somatic soluble proteins (i.e. S3 fraction) cannot signal to classical Ras proteins.

It is important to note that it cannot be determined by this method, if Rasgrp1 is predominantly pre- or postsynaptically localized. In the fraction containing synaptic plasma membranes (LP1B), proteins from both synaptic sides are found. Though weak, a signal can be detected in the fraction containing synaptic vesicle proteins (LP2). These results confirm a localization of Rasgrp1 to endomembranes. In addition they indicate, that Rasgrp1 is also found in fractions containing synaptic markers. 


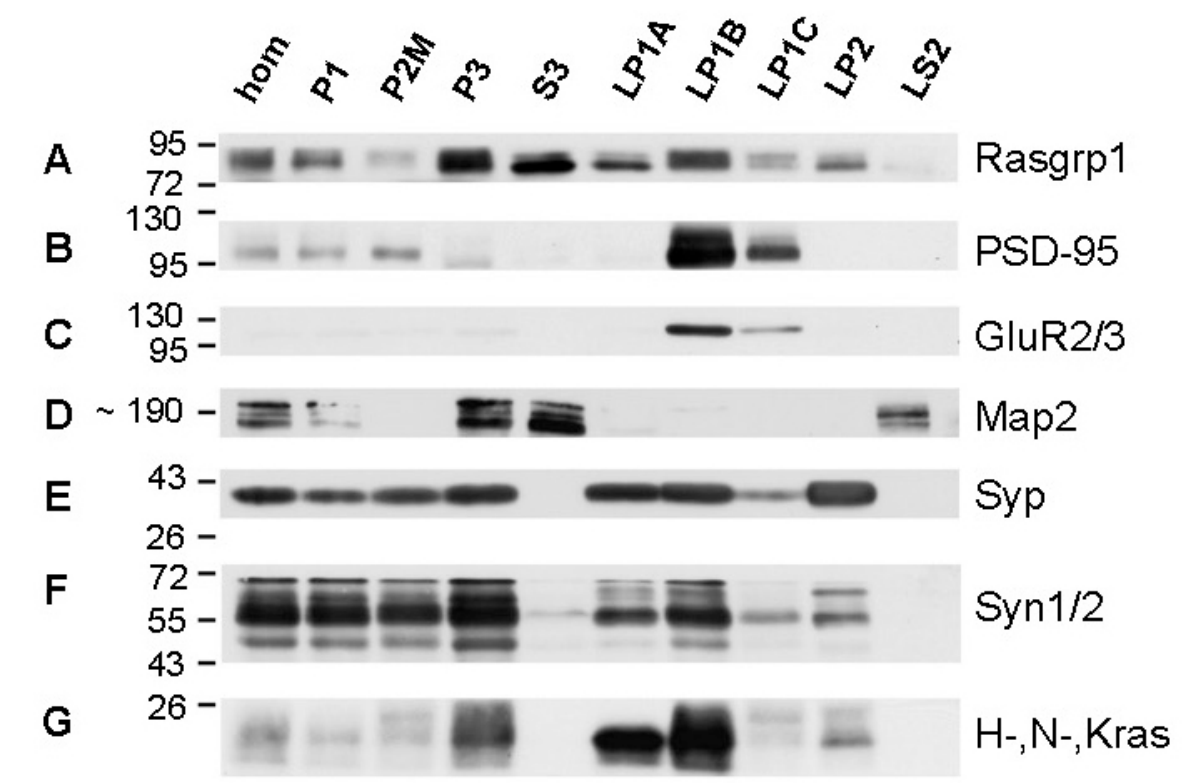

Figure V.4: Fore brain subcellular fractions containing Rasgrp1

Western blots of homogenate (hom) and subcellular fractions prepared from mouse forebrain were immunolabeled for Rasgrp1 and marker proteins.

(A, D) Strongest enrichment of Rasgrp1 was found in the microsomal fraction (P3) containing endomembranous proteins, followed by the S3 fraction, containing somatic soluble proteins, such as Map2. (B, C, E, F) Enrichment of Rasgrp1 was also detected in the fraction containing synaptic plasma membranes (LP1B), which was also positively immunolabeled for postsynaptic and presynaptic markers. (E, F) Rasgrp1 was present, but not enriched (as compared to homogenate), in the fractions containing synaptic vesicle proteins. (G) Classical Ras G proteins were found to be enriched in the same fractions as Rasgrp1.

Protein mass is indicated in $\mathrm{kDa}$ on the left. 


\section{V.2. Characterization of the Rasgrp1 KO Mouse}

The Rasgrp1 KO mouse line was generated by Dr. Andrea Betz (see VIII.1).

\section{V.2.1. No Obvious Behavioral Phenotypic Changes or Defects in Rasgrp1 KO Mice}

Heterozygous Rasgrp1 +/- animals were healthy and reproduced normally. Resulting Rasgrp1 $\mathrm{KO}$ animals developed normally and could not be distinguished from their WT littermates by appearance (Figure V.5 A). Animals between 3 and 8 weeks of age did not exhibit any overt obvious behavioral abnormalities. Prepared brains did not show differences in appearance or weight as compared to WT controls $\left(\mathrm{WT}_{\text {mean }}=0.515 \pm 0.046 \mathrm{~g} ; \mathrm{KO}_{\text {mean }}=0.487 \pm 0.045 \mathrm{~g} ; \mathrm{n}=6\right.$ pairs; $p=0.391$ ) (Figure V.5 B, C). These results indicate that Rasgrp1 is not crucially required for survival, growth, or brain development.

\section{V.2.2. Gross Brain Morphology of Rasgrp1 KO Mice is Normal}

Expression of constitutively activated Hras in the Synras mouse led to general neuronal hypertrophy, resulting in a strikingly increased cortical volume $(\sim 15 \%)$ (Heumann et al., 2000). The opposite effect may be expected in a mouse model exhibiting less potential to activate the Ras signaling pathway, such as the Rasgrp1 $\mathrm{KO}$. To test this, brain sections of KO and WT mice were analyzed by staining for Synapsin1/2 and by DAPI staining. When immunostained for Synapsin1/2, the laminae of the hippocampus can be clearly distinguished (Figure V.6 A). Synapsin stainings of Rasgrp1 and WT coronal sections were used to quantify the diameters of the cortex and the laminae of the hippocampus. No significant differences were found when comparing Rasgrp1 $\mathrm{KO}$ and $\mathrm{WT}$ mean diameters: $\mathrm{WT}_{\mathrm{ctx}}=753 \pm 28.7 \mu \mathrm{m} ; \mathrm{KO}_{\mathrm{ctx}}$ $=734 \pm 15.6 \mu \mathrm{m} ; p=0.609 ; \mathrm{WT}_{\mathrm{so}}=107 \pm 6.8 \mu \mathrm{m} ; \mathrm{KO}_{\mathrm{so}}=106 \pm 5.0 \mu \mathrm{m} ; p=0.888$; $\mathrm{WT}_{\mathrm{sp}}=33 \pm 2.2 \mu \mathrm{m} ; \mathrm{KO}_{\mathrm{sp}}=35 \pm 2.6 \mu \mathrm{m} ; p=0.635 ; \mathrm{WT}_{\mathrm{sr}}=167 \pm 12.4 \mu \mathrm{m} ; \mathrm{KO}_{\mathrm{sr}}=$ $176 \pm 11.6 \mu \mathrm{m} ; p=0.641 ; \mathrm{WT}_{\mathrm{slm}}=88 \pm 10.7 \mu \mathrm{m} ; \mathrm{KO}_{\mathrm{slm}}=95 \pm 5.5 \mu \mathrm{m} ; p=0.602 ; n=$ 6 pairs each (Figure V.6 B). When stained for Rasgrp1, no immunoreactivity was detected in coronal sections of KO mice (Figure V.6 C). In corresponding WT sections, a strong immunoreactivity was detected in the CA1 and the CA3 regions, but not in the dentate gyrus. Of all regions expressing Rasgrp1, strongest immunofluorescence is found in the CA3 region. 

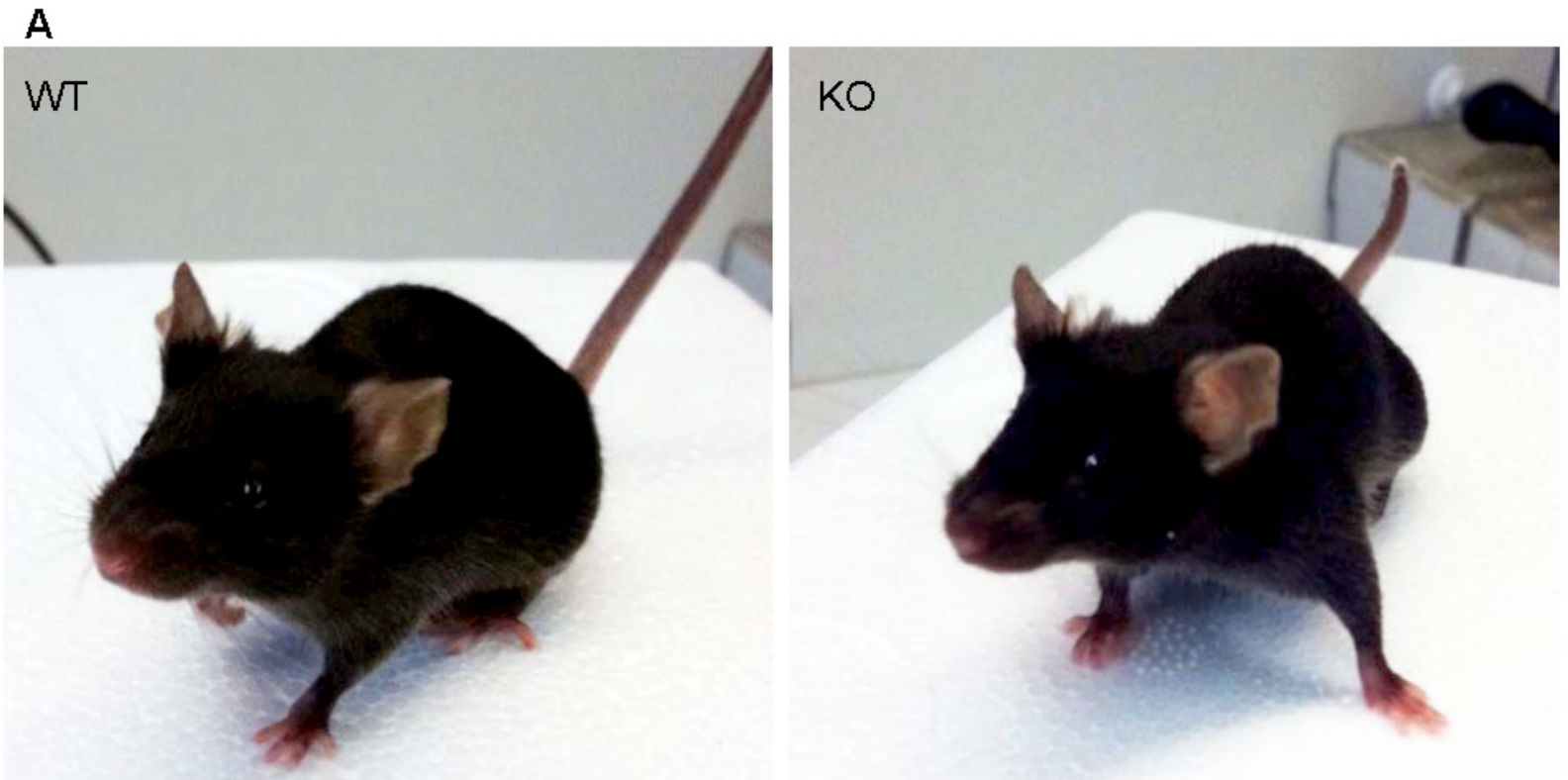

B

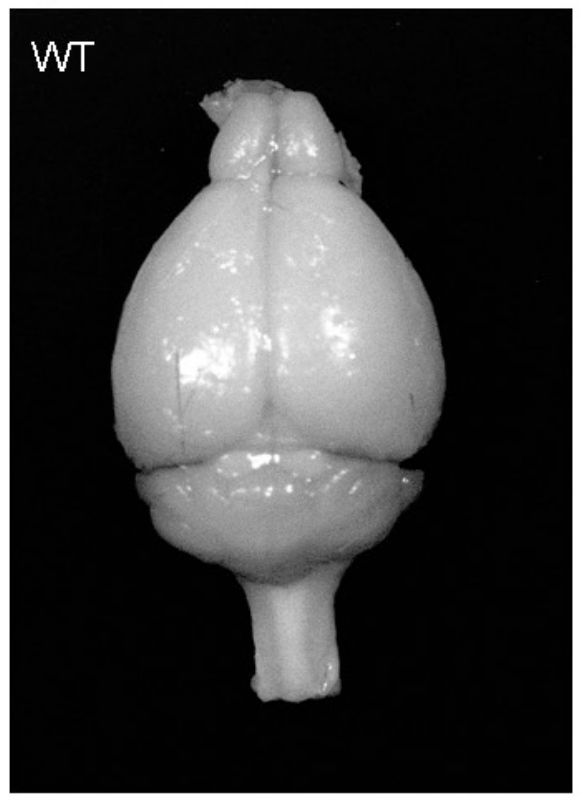

\section{$\mathrm{KO}$}

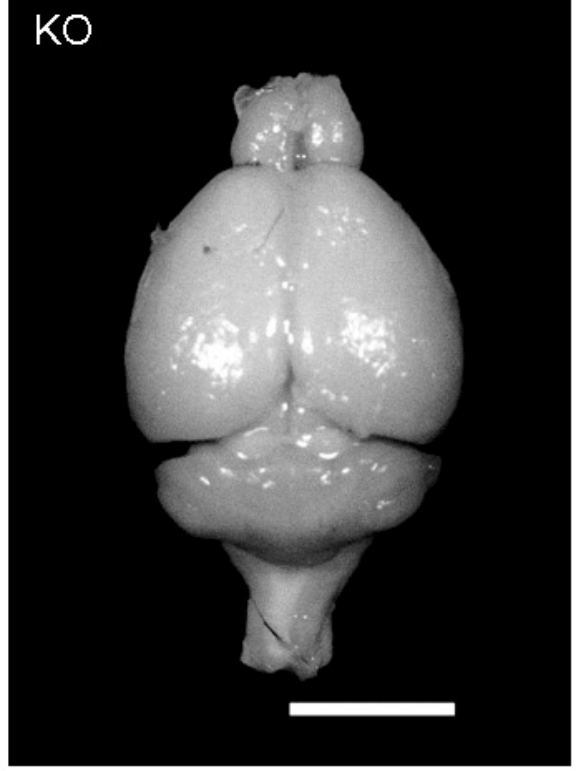

C

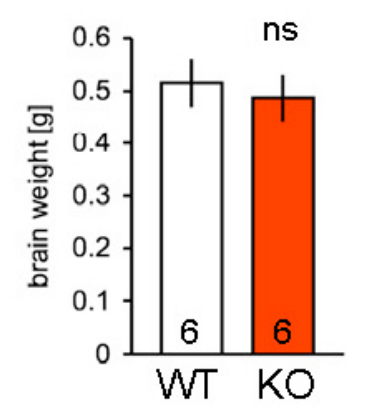

Figure V.5: Observation of Rasgrp1 KO mice and brains

Adult Rasgrp1 knock-out (KO) mice did not show obvious behavioral phenotypic changes or defects in brain structure.

(A) Two female littermates of the indicated genotypes at the age of 5 weeks (w). (B) Brains were prepared from 13 w old littermates. The KO brains observed did not show any obvious defect. Scale bar $=5 \mathrm{~mm}$. (C) The mean weight of KO brains was not significantly different from that of wild-type (WT) brains. Numbers $(n)$ of brains analyzed are shown at the bottom of the bar graphs. Error bars represent standard deviation ( $s d)$. Statistical analysis was carried out using the two-tailed Student's $t$-test (ns, not significant). 
A

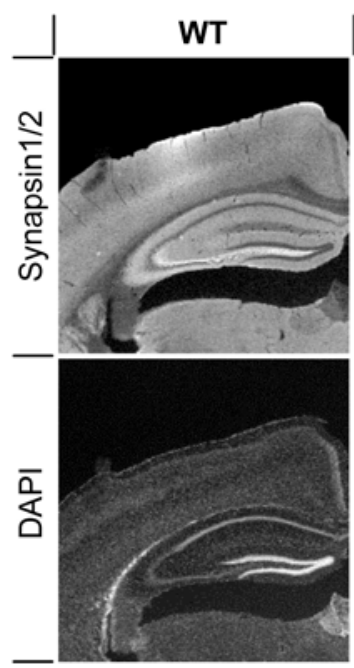

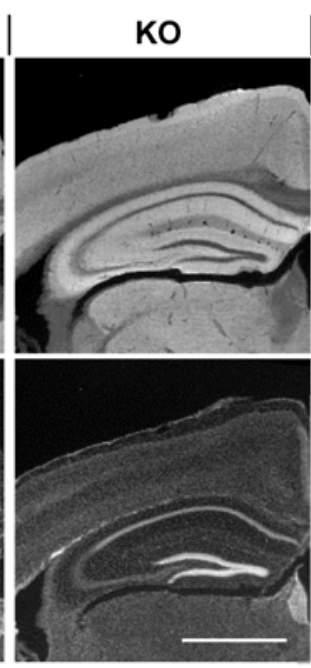

B

C
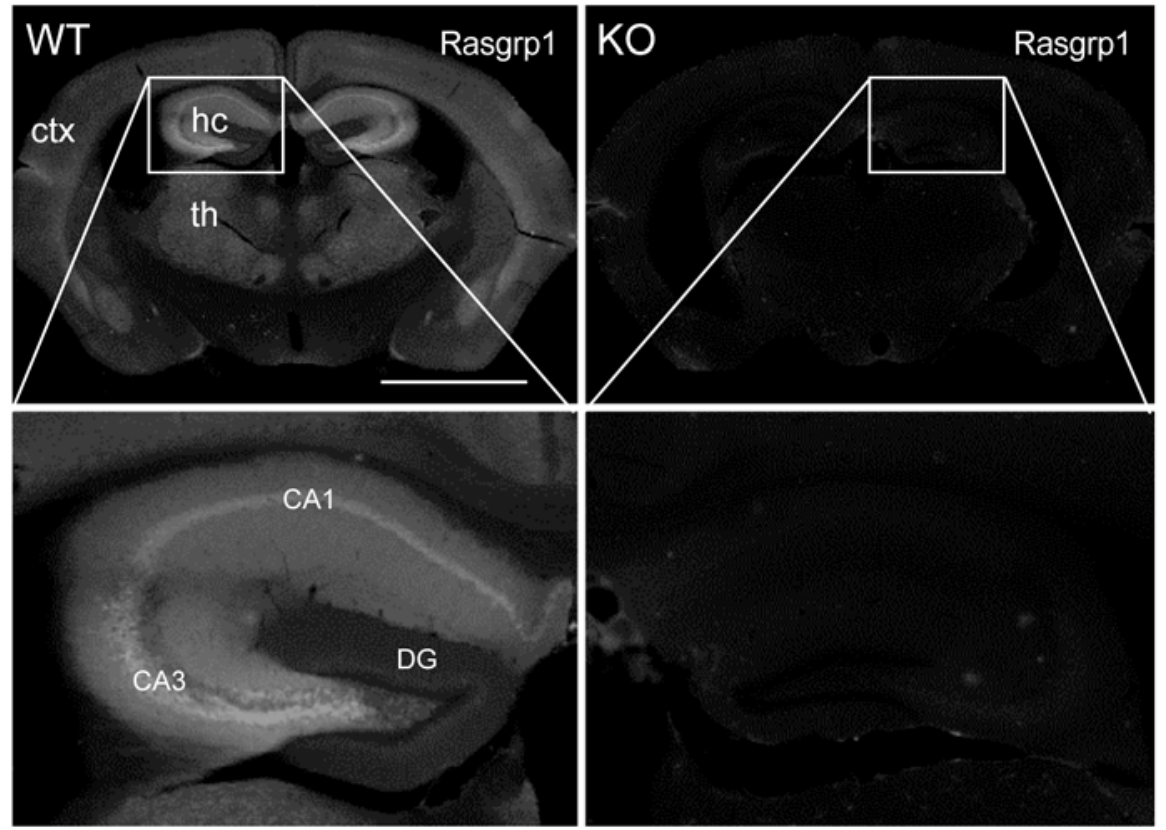

\section{Figure V.6: Gross brain morphology of Rasgrp1 KO mice}

Histochemical stainings of coronal brain sections were used to study morphological properties in Rasgrp1 knock-out (KO) mice and the expression pattern of Rasgrp1 in wild-type (WT) mice.

(A) Immunohistochemical stainings for Synapsin 1/2 (Syn) and DAPI stainings revealed no obvious changes in gross morphology of sections prepared from 3-5 week (w) old KO mice. Scale bar $=1 \mathrm{~mm}$. (B) No significant differences were found in diameters of Rasgrp1 KO brain regions as compared to WT. Error bars represent sem; two tailed student's $t$-test, $n=6$ pairs of littermates; ns, not significant; ctx, cortex; so, stratum oriens; sp, stratum pyramidale; sr, stratum radiatum; slm, stratum lacunosum moleculare. (C) Rasgrp1 immunoreactivity was most prominent in the cortex, in thalamic nuclei (th), and in the hippocampus (hc) of $8 \mathrm{w}$ old WT mice. In the 
hc, immunoreactivity was detected in the Cornu Ammonis region 1 (CA1) and in CA3, but not in the dentate gyrus (DG). Deletion of Rasgrp1 and specificity of the antibody were verified by the absence of immunoreactivity in the corresponding $\mathrm{KO}$ coronal sections. Scale bar $=2 \mathrm{~mm}$. 


\section{V.2.3. Rasgrp1 KO Neurons Display Aberrant Dendrite Morphogenesis}

The possibility of an involvement of Rasgrp1 function in dendrite morphogenesis was investigated in detail, because previously published findings indicate a positive role of Ras signaling in this process (Alpar et al., 2003; Arendt et al., 2004; Gärtner et al., 2005; Holzer et al., 2001; Jaworski et al., 2005; Kumar et al., 2005; Seeger et al., 2003) and because Rasgrp1 overexpression in PC12 cells leads to neurite outgrowth (Bivona et al., 2003).

Dendritic development of hippocampal neurons in-vivo begins at postnatal day 5 (P5). Starting at P5, dendritic complexity increases rapidly and only slows down after P15 (Pokorny and Trojan, 1986). RasGRP1 expression starts at P2 and reaches an adult-like pattern around P20 (Pierret et al., 2000). Thus, the increase in dendritic complexity and the increase of RasGRP1 expression correlate during the initial phase of dendrite morphogenesis. In cultured rat hippocampal neurons, the increase of dendritic complexity is most rapid between DIV4 and DIV10 and resembles a sigmoidal curve if complexity is taken as a measurement (Dotti et al., 1988). This time period is characterized by extensive growth and branching of neurites. However, dendritic development also requires regressive events that constrain dendritic expansion. In mature stages, the final morphology is shaped by a dynamic balance between growth and elimination of individual dendritic protrusions (Wong and Ghosh, 2002).

Hippocampal Rasgrp1 KO neurons were prepared from P0 animals and cultured as described (chapter IV.3.1). Cultures were fixed and stained for Map2 at DIV5, DIV10, DIV14 and DIV21. Analyses of dendritic complexity were carried out using the method described by Sholl et al. (1953). Neurons grown in continental cultures were used for analyses at DIV5 and DIV10. As neuron density and resulting overlap of dendritic processes increases after DIV10, neurons grown separately on astrocyte islands were used for analyses of dendritic morphology at DIV14 and DIV21.

Upon cursory observation, DIV5 neurons from KO or WT cultures did not show an obvious difference. However, Sholl analysis of 85 neurons of each genotype $(N=$ 4 different cultures prepared from littermates) showed slightly reduced dendritic complexity of Rasgrp1 KO neurons (Figure V.7 A). The difference in intersections was significant in medial and distal segments $\left(\mathrm{WT}_{40}=5.2 \pm 0.255 ; \mathrm{KO}_{40}=4.25 \pm\right.$ 
$\left.0.254 ;{ }^{* *} p=0.009 ; \mathrm{WT}_{80}=1.33 \pm 0.193 ; \mathrm{KO}_{80}=0.86 \pm 0.127 ;{ }^{*} p=0.045\right)$. When DIV10 neurons were observed ( $n, \mathrm{WT}=73 ; n, \mathrm{KO}=72 ; N=3$ cultures each), no difference in dendritic complexity was measured by Sholl analysis (Figure V.7 B).

In contrast to the reduced complexity at DIV5, neurons showed an enhanced dendritic complexity in medial and distal regions at DIV14 ( $n, \mathrm{WT}=39 ; n, \mathrm{KO}=40$; $N=3$ cultures each). The number of intersections was significantly different at 150 and $175 \mu \mathrm{m}$ distance from the soma $\left(\mathrm{WT}_{150}=0.61 \pm 0.316 ; \mathrm{KO}_{150}=1.74 \pm 0.414 ;{ }^{*} p\right.$ $\left.=0.025 ; \mathrm{WT}_{175}=0.22 \pm 0.126 ; \mathrm{KO}_{175}=0.78 \pm 0.337 ;{ }^{*} p=0.046\right)$. When observed qualitatively, this difference was due to an extended dendritic territory of neurons and not to increased branching in distal regions. This indicates an increase in complexity by elongated morphology of dendrites (Figure V.7 C). This phenotype was also observed in DIV21 neurons ( $n, \mathrm{WT}=37 ; n, \mathrm{KO}=50 ; N=2$ cultures each). Interestingly, the number of intersections measured up to $75 \mu \mathrm{m}$ distance from the soma differed even less than between WT and KO neurons at DIV14, resulting in virtually overlapping Sholl plots (Figure V.7 D). However, at 125, 150, 175 and 200 $\mu \mathrm{m}$ distance, numbers of intersections were significantly higher in KO cells $\left(\mathrm{WT}_{125}=\right.$ $1.05 \pm 0.412 ; \mathrm{KO}_{125}=2.07 \pm 0.349 ;{ }^{*} p=0.035 ; \mathrm{WT}_{150}=0.33 \pm 0.194 ; \mathrm{KO}_{150}=1.77 \pm$ $0.399 ;{ }^{* *} p=0.002 ; \mathrm{WT}_{150}=0.33 \pm 0.194 ; \mathrm{KO}_{150}=1.77 \pm 0.399 ;{ }^{* *} \mathrm{p}=0.002 ; \mathrm{WT}_{175}=$ $0.00 \pm 0.0 ; \mathrm{KO}_{175}=0.8 \pm 0.280 ;{ }^{* *} p=0.003 ; \mathrm{WT}_{200}=0.000 \pm 0.0 ; \mathrm{KO}_{200}=0.37 \pm$ $0.179 ;{ }^{*} p=0.026$ ).

These results indicate that lack of Rasgrp1 in early dendrite morphogenesis leads to a decrease in dendritic complexity. Conversely, with regard to late dendrite morphogenesis and mature morphology, Rasgrp1 KO neurons show an increased dendritic complexity. 
A
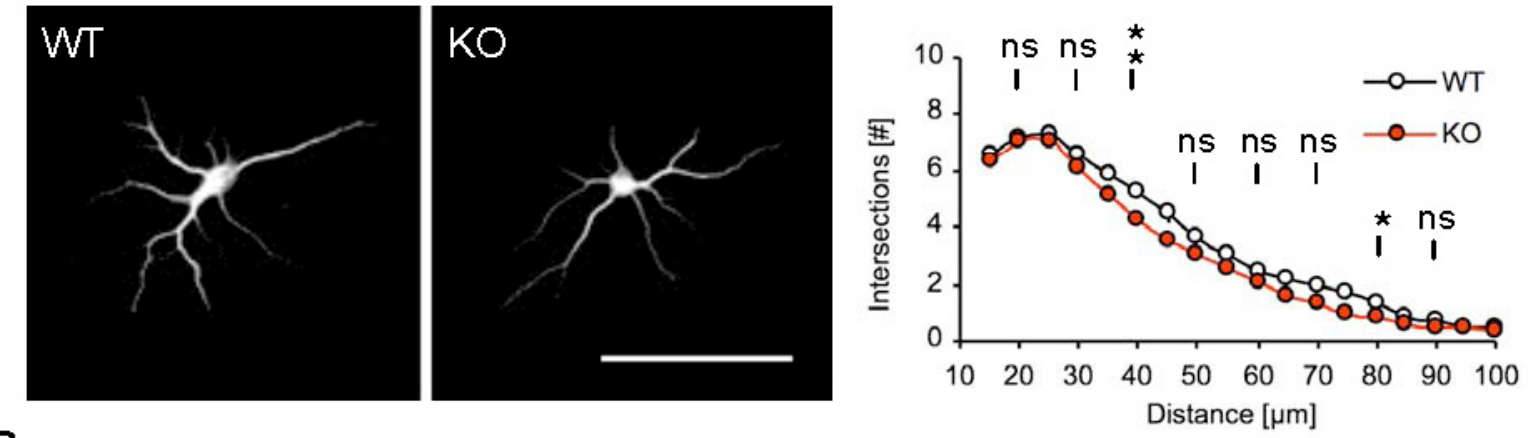

B
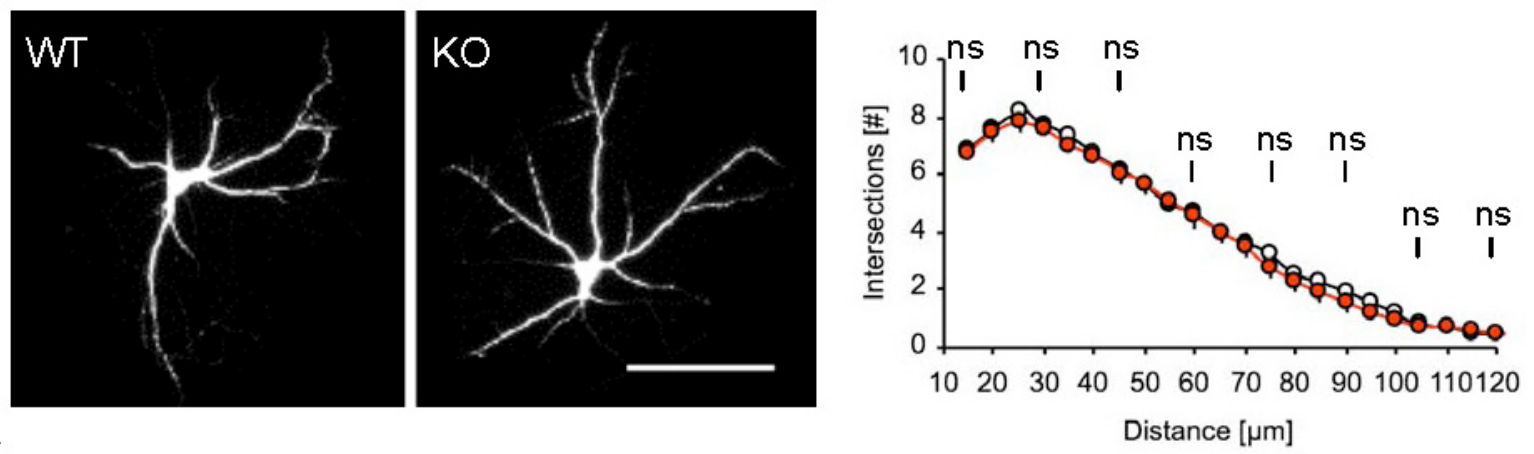

C
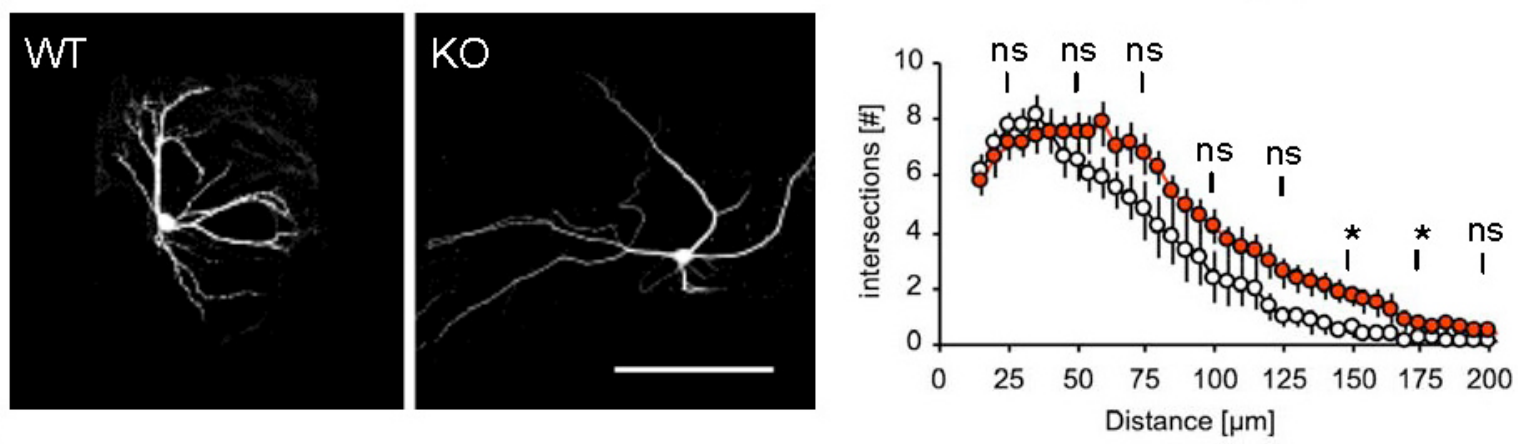

D
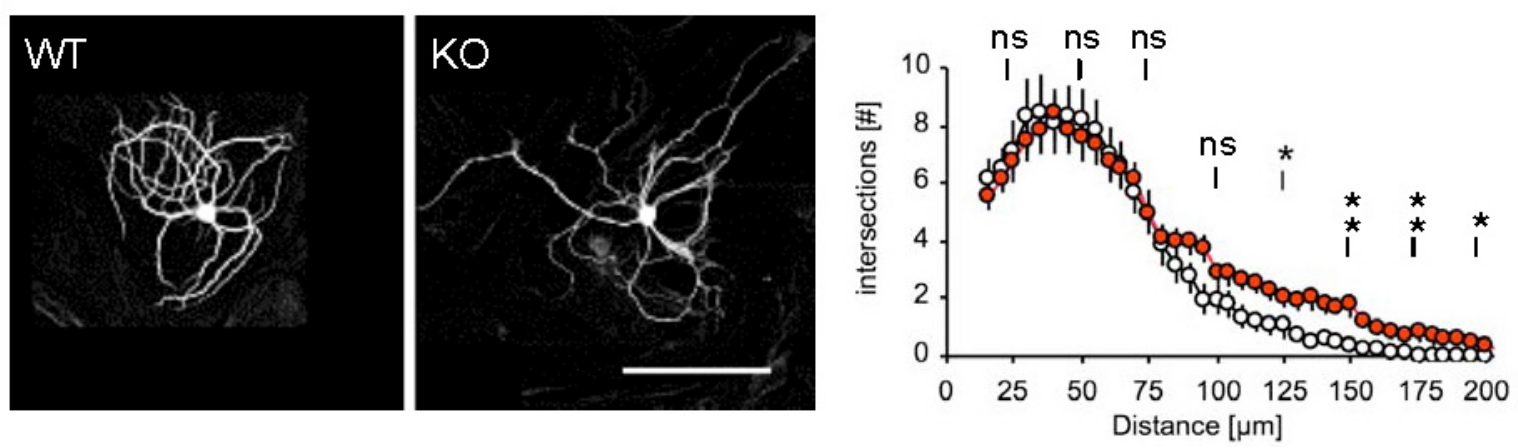

Figure V.7: Analysis of dendrite morphogenesis in Rasgrp1 KO neurons

Dendrite morphogenesis of cultured hippocampal neurons was investigated using Sholl analysis (circle interval = $5 \mu \mathrm{m}$ ) after Map2 immunostaining. Rasgrp1 knock-out (KO) neurons display a defect in dendrite morphogenesis. Sample neurons of different developmental stages stained for Map2 are shown next to the corresponding Sholl plot. The indicated values were statistically analyzed using Students $t$-test. (A) Dendrite morphology at day in-vitro (DIV) 5; scale bar $=100 \mu \mathrm{m}$. KO neurons showed a slightly, but significantly decreased dendrite complexity ( $n$, WT 
$=85 ; n, \mathrm{KO}=85)$. (B) Dendrite morphology of cultures at DIV 10. No significant differences in $\mathrm{KO}$ dendrite morphology were observed as compared to wild-type (WT) neurons ( $n, \mathrm{WT}=73 ; n, \mathrm{KO}=72$ ); scale bar $=50 \mu \mathrm{m}$. (C) Dendrite morphology at DIV $14(n, \mathrm{WT}=29 ; n, \mathrm{KO}=40)$; scale bar $=150 \mu \mathrm{m}$. (D) Dendrite morphology at DIV 21 ( $n, \mathrm{WT}=37 ; n, \mathrm{KO}=50$ ); scale bar $=150 \mu \mathrm{m}$. (C, D) At DIV 14 and DIV 21, Rasgrp1 KO neurons show an significantly enhanced dendrite complexity in distal regions. Neurons analyzed in (A) and (B) were grown in continental cultures, and neurons analyzed in (C) and (D) were grown on astrocyte islands. Error bars represent sem. All statistical analyses were carried out using the two-tailed Student's $t$-test; ${ }^{*}, p<0.05 ;{ }^{* *}, p<0.01$; ns, not significant. 


\section{V.2.4. Rasgrp1 KO Mice Show an Increased Synaptic Transmission}

\section{V.2.4.1. Field Recordings in the Rasgrp1 KO Hippocampus}

While Ras signaling clearly regulates physiological functions in neurons, its exact role is far from clear (see chapter II.3). For example, decreasing the potential to activate Ras by a KO of the Ras GEF Rasgrf1 leads to enhanced basal transmission (Brambilla et al., 1997), but the same effect is observed upon increases of Ras activity by overexpression of Hras in hippocampal neurons (Zhu et al., 2002).

To investigate the function of Rasgrp1 in synaptic transmission, acute hippocampal slices from Rasgrp1 KO mice were stimulated in the stratum radiatum of the CA1 region. The amplitude of the resulting fiber volley ( $\mathrm{fv}$ ) of the Schaffer collaterals is generally assumed to reliably correlate to presynaptic stimulation. Therefore, stimulation was set to obtain $\mathrm{fv}$ amplitudes at increasing intervals of 0.05 $\mathrm{mV}(0.05,0.10 .15,0.2,0.25$ and $0.3 \mathrm{mV})$. The resulting field excitatory postsynaptic potentials (fEPSPs) were recorded further medially in the CA1 stratum radiatum. The output signals (mean fEPSP slopes/amplitudes) were plotted relative to the correlating input signals ( $\mathrm{fv}$ amplitudes) (Figure IV.1 A). Rasgrp1 KO fEPSPs exhibited significantly increased mean slopes and amplitudes (see Table V.1), indicating enhanced efficiency of synaptic transmission.

Table V.1: Input-output experiments in Rasgrp1 KO hippocampal slices

\begin{tabular}{|c|c|c|c|c|c|c|c|c|c|c|}
\hline \multirow{3}{*}{$\begin{array}{c}\mathrm{fV} \\
0.05\end{array}$} & \multicolumn{5}{|c|}{ fEPSP slope } & \multicolumn{5}{|c|}{ fEPSP amplitude } \\
\hline & \multicolumn{2}{|c|}{ WT } & \multicolumn{2}{|c|}{$\mathrm{KO}$} & \multirow{2}{*}{$\frac{p}{0.006^{* *}}$} & \multicolumn{2}{|c|}{ WT } & \multicolumn{2}{|c|}{$\mathrm{KO}$} & \multirow{2}{*}{$\begin{array}{c}p \\
0.048^{*}\end{array}$} \\
\hline & 0.08 & \pm 0.008 & 0.12 & \pm 0.009 & & 0.21 & \pm 0.023 & 0.29 & \pm 0.027 & \\
\hline 0.1 & 0.14 & \pm 0.016 & 0.22 & \pm 0.025 & $0.02^{*}$ & 0.38 & \pm 0.039 & 0.54 & \pm 0.054 & $0.03^{*}$ \\
\hline 0.15 & 0.21 & \pm 0.030 & 0.31 & \pm 0.030 & $0.048^{*}$ & 0.56 & \pm 0.069 & 0.70 & \pm 0.061 & 0.169 \\
\hline 0.2 & 0.25 & \pm 0.029 & 0.39 & \pm 0.029 & $0.064^{* *}$ & 0.68 & \pm 0.079 & 0.91 & \pm 0.069 & 0.047 \\
\hline 0.25 & 0.30 & \pm 0.034 & 0.48 & \pm 0.030 & $0.013^{* *}$ & 0.77 & \pm 0.085 & 1.15 & \pm 0.088 & $0.093^{\star *}$ \\
\hline 0.3 & 0.33 & \pm 0.038 & 0.54 & \pm 0.036 & $0.012^{\star *}$ & 0.84 & \pm 0.080 & 1.33 & \pm 0.111 & $0.003^{* *}$ \\
\hline
\end{tabular}

The first column displays the fiber volley (fv) amplitude [mV]. Slope values are given in $\mathrm{mV} / \mathrm{ms} \pm$ sem; amplitudes in $\mathrm{mV} \pm$ sem; $n, \mathrm{WT}=11$ slices; $n, \mathrm{KO}=12$ slices. Statistical analyses were carried out using the two-tailed Students $t$-test ; ${ }^{*}, p<0.05 ;{ }^{* *}, p<0.01$.

Next, LTP induction was tested in Rasgrp1 KO hippocampi (Figure V.8 B). Four tetanic stimuli were applied in $20 \mathrm{~s}$ intervals after recording a stable baseline for 10 min. The resulting mean potentiation shortly after these stimuli (binned values from 11 to $15 \mathrm{~min}$ ) was $1.61 \pm 0.054$ fold baseline in WT mice and $1.40 \pm 0.051$ fold baseline in $\mathrm{KO}$ mice. The difference was statistically significant $\left({ }^{*} p=0.018\right)$. The 
binned mean values from 16-20 $\mathrm{min}\left(\mathrm{WT}_{16-20}=1.32 \pm 0.038 ; \mathrm{KO}_{16-20}=1.20 \pm 0.040\right)$ also showed a significantly lower potentiation in $\mathrm{KO}$ animals $\left({ }^{*} p=0.047\right)$. However, at the end of the recording period (averaged values from 36 to $40 \mathrm{~min}$ ), no significant differences were detected, although potentiation was still lower in $\mathrm{KO}$ animals $\left(\mathrm{WT}_{16}\right.$ $\left.20=1.33 \pm 0.040 ; \mathrm{KO}_{16-20}=1.23 \pm 0.046 ; p=0,131 ; n, \mathrm{WT}=11 ; n, \mathrm{KO}=12\right)$.

Increased basal transmission and reduced LTP in Rasgrp1 KO animals could result from pre- or postsynaptic effects. A series of experiments were carried out to test the presynaptic release probability for neurotransmitters in Rasgrp1 KO slices. First, paired pulse facilitation (PPF), i.e. the enhancement of neurotransmitter release in response to two closely spaced stimuli, was tested at different inter-stimulus intervals (ISI) (Figure V.8 C). The first stimulus drives calcium into the presynapse, some of which remains as the second stimulus leads to additional calcium influx. Upon the second stimulus, the increased calcium concentration triggers a larger number of synaptic vesicles to fuse with the presynaptic membrane in Schaffer collaterals contacting CA1 pyramidal cells, resulting in enhanced neurotransmitter release (Zucker and Regehr, 2002). This facilitation of release is detectable as a larger fEPSP following the second stimulus. When comparing genotypes, a change in PPF would therefore indicate a likely defect in the presynaptic vesicle release machinery. However, at all the intervals tested (20, 50, 100, 200, 300, 400 and 500 ms ISI), the mean facilitation was not significantly different between WT and KO animals (Table V.2).

Table V.2: Paired pulse facilitation in Rasgrp1 KO hippocampal slices

\begin{tabular}{|c|cc|cc|c|}
\hline ISI & \multicolumn{2}{|c|}{ WT } & \multicolumn{2}{|c|}{ KO } & $\boldsymbol{p}$ \\
\hline $\mathbf{2 0}$ & 1.23 & \pm 0.057 & 1.21 & \pm 0.057 & 0.775 \\
\hline $\mathbf{5 0}$ & 1.17 & \pm 0.044 & 1.14 & \pm 0.044 & 0.587 \\
\hline $\mathbf{1 0 0}$ & 1.12 & \pm 0.034 & 1.10 & \pm 0.034 & 0.451 \\
\hline $\mathbf{2 0 0}$ & 1.08 & \pm 0.028 & 1.05 & \pm 0.028 & 0.202 \\
\hline $\mathbf{3 0 0}$ & 1.02 & \pm 0.022 & 1.00 & \pm 0.022 & 0.45 \\
\hline $\mathbf{4 0 0}$ & 0.98 & \pm 0.022 & 0.97 & \pm 0.022 & 0.533 \\
\hline $\mathbf{5 0 0}$ & 0.95 & \pm 0.015 & 0.94 & \pm 0.015 & 0.482 \\
\hline
\end{tabular}

The first column displays the inter-stimulus intervals at which PPF was tested. Values are given as the ratio of the second to the first amplitude, $n, \mathrm{WT}=11$ slices; $n, \mathrm{KO}=12$ slices. Statistical analyses were carried out using the two-tailed Students $t$-test.

Paired pulse facilitation is a correlate of presynaptic short-term facilitation. However, synapses with high basal levels of transmission and high basal release probability exhibit paired-pulse depression as preceding stimuli deplete the pool of 
releasable vesicles to a degree that even the elevated calcium concentrations cannot cause an increased release. Reduction of basal transmission, e.g. by reducing calcium concentration, relieves depression (Zucker and Regehr, 2002). The standard Ringer's solution used in the experiments described above contained $2.5 \mathrm{mM}$ calcium. Additional PPF experiments at low calcium concentration (1.5 mM; $50 \mathrm{~ms}$ ISI) were performed in order to determine if the loss of Rasgrp1 causes changes in presynaptic function when release probability is low (Figure V.8 D). However, no significant difference was detected in mean ratios of WT and KO animals (WT $=1.74$ $\pm 0.044 ; \mathrm{KO}=1.79 \pm 0.044 ; n, \mathrm{WT}=10 ; n, \mathrm{KO}=10 ; p=0.679)$. To further examine longer lasting forms of short-term plasticity, $14 \mathrm{~Hz}$ train experiments were conducted. During repetitive stimulation of synapses at this moderate frequency, control synaptic responses at Schaffer collateral- CA1 synapses show an initial facilitation, followed by depression (Schoch et al., 2002). $14 \mathrm{~Hz}$ train experiments were performed at both normal and reduced calcium concentrations (Figure V.8 E, F). At normal calcium concentration, a strong depression (ratio of last to first fEPSP) was detected in both WT and in Rasgrp1 KO slices (Figure V.8 E). However, the genotypes did not differ (Table V.3). Facilitation at $1.5 \mathrm{mM}$ Calcium was sustained even at the last stimulus in control slices, indicating that this concentration does not lead to depletion of synaptic vesicles during stimulation (Figure V.8 F). Again, comparative analysis of responses in WT and Rasgrp1 KO did not reveal significant differences (Table V.3).

Table V.3: $14 \mathrm{~Hz}$ experiments in Rasgrp1 KO hippocampal slices

\begin{tabular}{|c|c|c|c|c|c|c|c|c|c|c|}
\hline & \multicolumn{5}{|c|}{$2.5 \mathrm{mM}$ Calcium (Figure V.8 E) } & \multicolumn{5}{|c|}{$1.5 \mathrm{mM}$ Calcium (Figure V.8 F) } \\
\hline ratio & \multicolumn{2}{|c|}{ WT } & \multicolumn{2}{|c|}{$\mathrm{KO}$} & \multirow{2}{*}{$\begin{array}{c}p \\
0.302\end{array}$} & \multicolumn{2}{|c|}{ WT } & \multicolumn{2}{|c|}{$\mathrm{KO}$} & $p$ \\
\hline $2^{\text {nd }} / 1^{\text {st }}$ & 1.30 & \pm 0.041 & 1.25 & \pm 0.015 & & 1.62 & \pm 0.043 & 1.68 & \pm 0.045 & 0.333 \\
\hline last $/ 1^{\text {st }}$ & 0.72 & \pm 0.066 & 0.60 & \pm 0.03 & 0.125 & 1.63 & \pm 0.143 & 1.65 & \pm 0.066 & 0.936 \\
\hline
\end{tabular}

Values are given as ratios between $2^{\text {nd }}$ and $1^{\text {st }}$ and last and $1^{\text {st }}$ fEPSP amplitude $\pm s e m ; n$, WT $=10$ slices; $n, \mathrm{KO}=10$ slices. Statistical analyses were carried out using the two-tailed Students $t$-test.

In summary, field recordings in the CA1 region of Rasgrp1 $\mathrm{KO}$ mice revealed a phenotype that characterized by an increased basal synaptic transmission and reduced potentiation in the early phases of LTP. In addition, experiments assessing presynaptic release probability and depletion of synaptic vesicles, did not show abnormal results in Rasgrp1 deficient mice. 
A
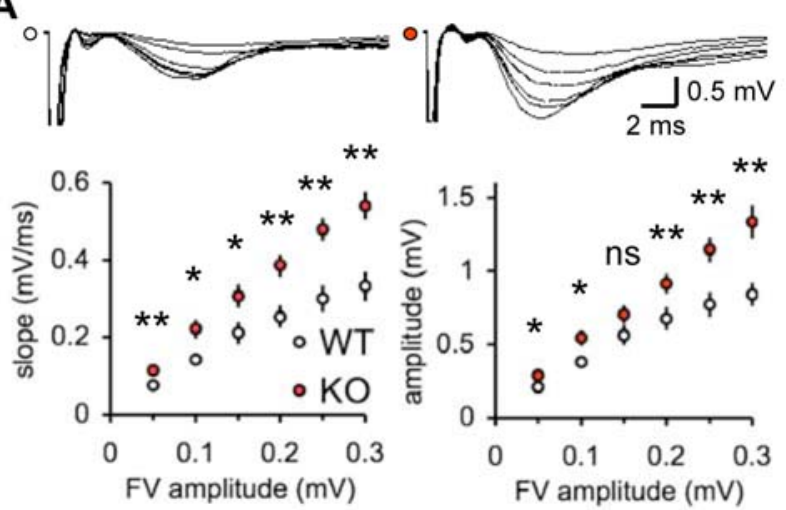

B
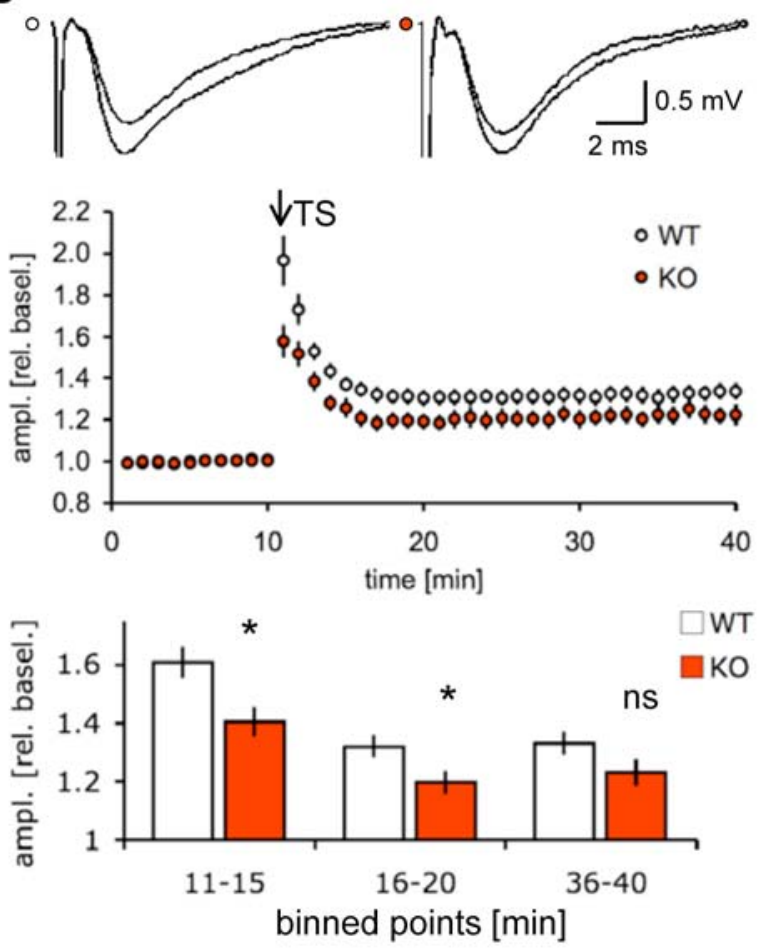

C
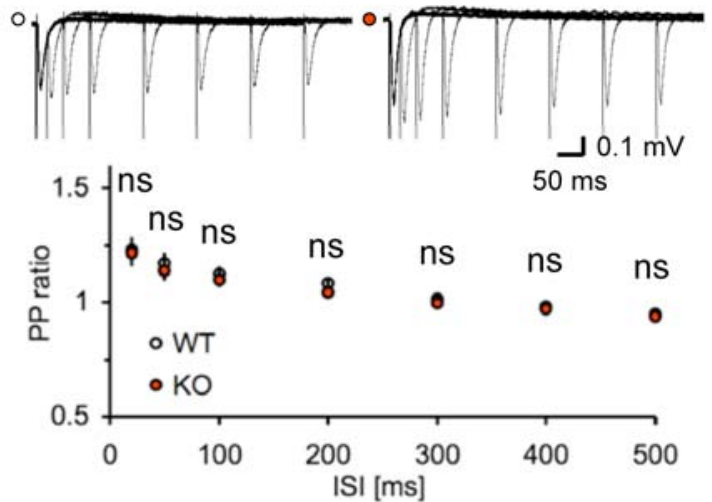

D

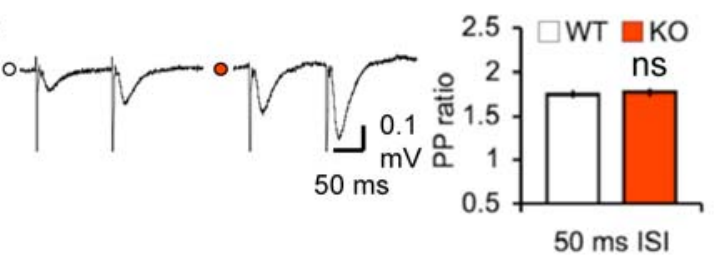

$\mathbf{E}$

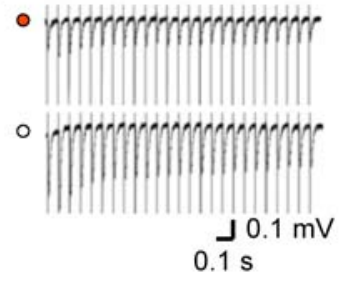

$\mathbf{F}$
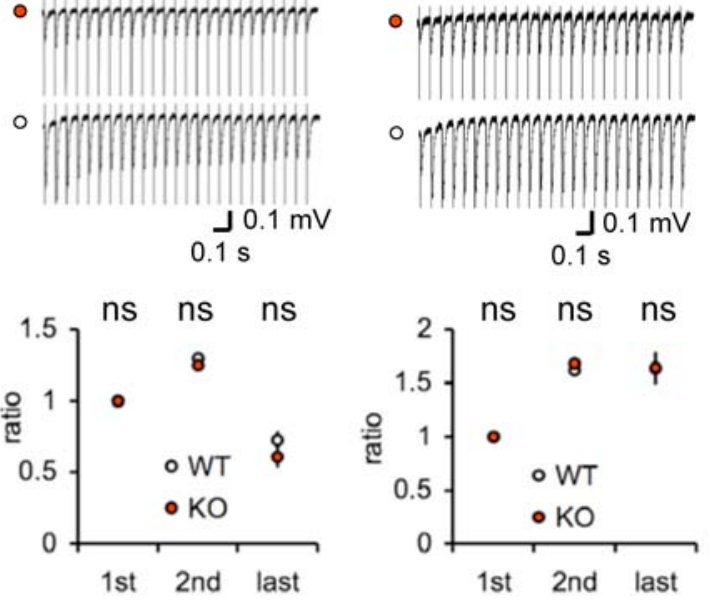

Figure V.8: Field recordings in the CA1 stratum radiatum of Rasgrp1 KO mice

(A) Rasgrp1 KO mice (3-5 weeks) showed an increased basal synaptic transmission, as seen in sample traces showing the input (fiber volley) and the output (fEPSP). Input-output curves for basal synaptic transmission were generated from the mean fEPSP slope (left panel) and amplitude (right panel) of 11 experiments in wild-type (WT) and 12 experiments in Rasgrp1 knock-out (KO) mice. (B) LTP was significantly impaired in KO mice. LTP was induced by tetanic stimulation (TS), i.e. 100 pulses at $100 \mathrm{~Hz}$, four times, $20 \mathrm{~s}$ apart. Traces on top of the plot are averages of 5 consecutive responses each, taken from the end of the baseline and from the end of the recording period. The summary plot shows the normalized mean amplitude of 11 experiments in WT and 12 experiments in KO. The bar graphs show the averaged 
values taken from the indicated time periods. (C) Paired pulse facilitation (PPF) was not significantly changed in KO mice. The traces on top of the plot are overlays of traces (each the average of 5 consecutive sample traces) recorded at inter-stimulus intervals (ISI) ranging from 20 to $500 \mathrm{~ms}$. The plot shows the ratio of second to first fEPSP (WT, $n=11$; KO, $n=12$ ). (D-F) Similarily, no significant differences were found in PPF (ISI = 50 ms) at low Calcium concentration (D), and in $14 \mathrm{~Hz}$ train experiments at normal $(\mathrm{E})$ or low $(\mathrm{F})$ Calcium concentration (WT, $n=10$; KO, $n=10$, each). In $14 \mathrm{~Hz}$ trains, the ratio of second to first and last to first amplitude was analyzed. Traces are averages of 5 consecutive sample recordings.

Error bars represent sem; all statistical analyses were carried out using the two-tailed Students $t$-test ; ${ }^{*}, p<0.05 ;{ }^{* *}, p<0.01 ;$ ns, not significant. 


\section{V.2.4.2. Patch Clamp Recordings in the Rasgrp1 KO Hippocampus}

Next, patch clamp recordings were performed to record mini excitatory postsynaptic currents (mEPSCs) and to assess the sensitivity of pyramidal neurons to bath application of L-AMPA. The recordings were obtained from neurons in the CA1 stratum pyramidale and performed in the presence of the sodium channel blocker tetrododoxin (TTX, $1 \mu \mathrm{M}$ ), and the AMPAR-desensitization inhibitor cyclothiazide (CTZ, $100 \mu \mathrm{M})$. Blocking of sodium channels abolishes action potential generation, which limits the amount of neurotransmitter released to the spontaneous fusion of single synaptic vesicles with the presynaptic membrane. Ideally, a mEPSC represents the effect of one "quantal size" (i.e. the neurotransmitter content of a single synaptic vesicle) on the postsynaptic cell. Predicted that synaptic vesicle filling with neurotransmitter is normal, mEPSC sizes (i.e. amplitudes) indicate postsynaptic sensitivity. The frequency of mEPSCs recorded in a period of time is generally thought to correlate to the number of functional synapses and to the number and release probability of fusion competent vesicles in synapses. An increase in mEPSC amplitudes (i.e. postsynaptic efficiency) and/or increased mEPSC frequency (i.e. number of synapses, number of fusion competent vesicles per synapse) would help to explain the enhanced synaptic transmission observed in Rasgrp1 KO animals.

mEPSC amplitudes were analyzed by cumulative frequency distribution and by calculating the mean amplitude. In case of the recorded events from Rasgrp1 KO mice, the cumulative frequency distribution was shifted towards higher values of amplitudes. A two-sample Kolmogorow-Smirnow test showed that this shift was highly significant ( $n=618$ events each; $D_{\max }=0.289 ;{ }^{* \star *} p<0,001$; Figure V.9 A). Correspondingly, mean amplitudes were significantly increased in $K O(W T=13.99 \pm$ $0.679 \mathrm{pA} ; \mathrm{KO}=18.93 \pm 1.31 \mathrm{pA} ; n, \mathrm{WT}=9$ cells; $n, \mathrm{KO}=8$ cells; ${ }^{* *} p=0.0056$; Figure V.9 B). However, mEPSC frequency appeared to be normal in Rasgrp1 KO animals $(\mathrm{WT}=0.83 \pm 0.116 \mathrm{~Hz} ; \mathrm{KO}=0.79 \pm 0.155 \mathrm{~Hz} ; n, \mathrm{WT}=9 ; n, \mathrm{KO}=8 ; p=$ 0.878 ; Figure V.9 C). These results indicate an increased postsynaptic sensitivity in Rasgrp1 KO animals. Postsynaptic efficiency is regulated by a multitude of cellular mechanisms. The most likely mechanism to be involved in regulating synaptic transmission is a change in the number or the composition of postsynaptic receptors. The most prevalent receptors mediating basal synaptic transmission at Schaffer collaterals to CA1 synapses are AMPA receptors (AMPARs) that open upon the binding of glutamate. 
L-AMPA is an analogue of glutamate and activates AMPARs within the postsynaptic membrane (Honoré et al., 1982). In the whole-cell mode, the current ( $\left.\mathrm{I}_{\text {AMPA }}\right)$ necessary to hold the cell at a potential of $-60 \mathrm{mV}$ was recorded (Figure V.9 D). After recording 5 min of baseline, the standard Ringer's solution was substituted by a Ringer's solution containing $25 \mathrm{nM}$ L-AMPA. As the remaining standard solution and the L-AMPA containing solution mix in the recording chamber, L-AMPA concentration rises. I IMPA rapidly increases, as channels open and higher current is necessary to hold the cell at a potential of $-60 \mathrm{mV}$. Interestingly, Rasgrp1 $\mathrm{KO}$ cells not only required a higher current than WT cells, but also showed an earlier onset of reaction towards wash-in of L-AMPA. The mean current value obtained from 8 to 10 min after onset of recording ( 3 min after begin of wash in), was significantly increased in Rasgrp1 KO cells $\left(\mathrm{WT}_{8-10}=735.21 \pm 99.36 \mathrm{pA} ; \mathrm{KO}_{8-10}=918.91 \pm 92.37 \mathrm{pA} ; n\right.$, $\left.\mathrm{WT}=9 ; n, \mathrm{KO}=10 ;{ }^{*} p=0.047\right)$. After $10 \mathrm{~min}$, the holding current in Rasgrp1 KO cells recovered quite rapidly, until matching WT levels at around 15 min $\left(\mathrm{WT}_{14-16}=\right.$ $1147.82 \pm 148.42 \mathrm{pA} ; \mathrm{KO}_{14-16}=933.28 \pm 122.28 \mathrm{pA} ; n, \mathrm{WT}=9 ; n, \mathrm{KO}=9 ; p=$ $0.931)$. These results clearly show an increased sensitivity towards L-AMPA in Rasgrp1 KO cells.

In summary, above patch clamp recordings showed an enhanced postsynaptic sensitivity of Rasgrp1 KO cells, which likely result from increased sensitivity towards glutamate. The unchanged frequency of mEPSCs excludes, that formation of new synapses, an "un-silencing" of usually silent synapses, or substantial changes in the number or the release probability of fusion competent synaptic vesicles takes place in Rasgrp1 KO cells. Therefore, the increased transmission in $\mathrm{KO}$ cells observed in field recordings is likely to result from strengthening of existing postsynapses by an overabundance or changed properties of AMPA receptors. 
A
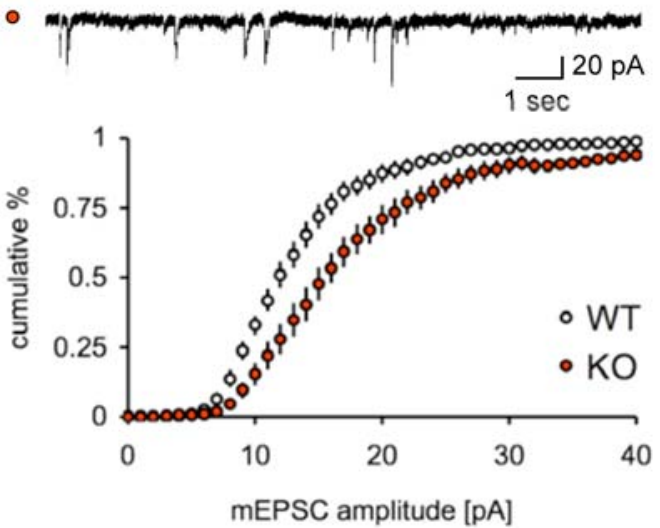

B

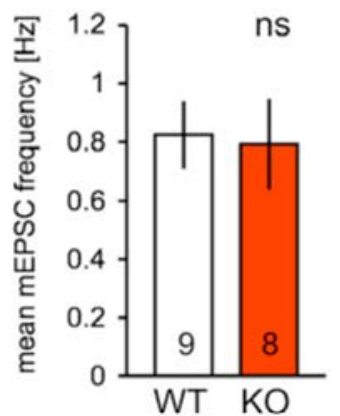

C



D

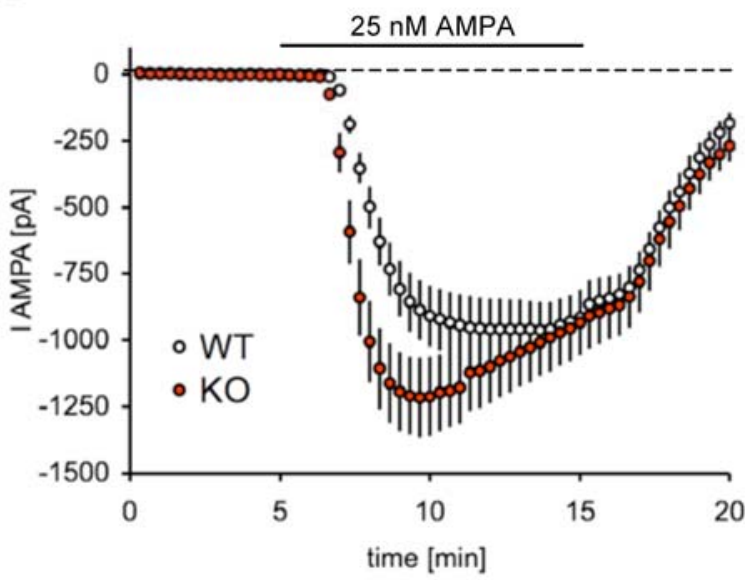

binned points [min]

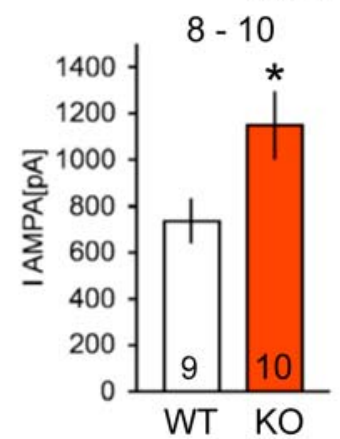

$14-16$

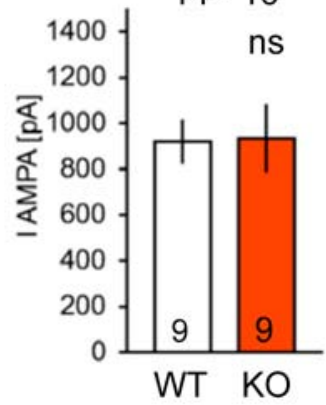

Figure V.9: Patch clamp recordings in the CA1 stratum pyramidale of Rasgrp1 KO mice

Rasgrp1 knock-out (KO) mice (2-4 weeks old) showed increased miniature synaptic current amplitudes and a higher sensitivity towards L-AMPA.

(A) Miniature EPSCs (mEPSCs) were recorded in whole cell mode at $-60 \mathrm{mV}$. Sample traces are shown on top. Cumulative frequency distribution displays a significant increase in mEPSC amplitudes of $\mathrm{KO}$ mice (two-sample KolmogorowSmirnow (KS)- test; 618 equally selected events each; ${ }^{* *} p<0.001 ; D_{\max }=0.289$ ). (B) The mean mEPSC frequency was unchanged in the KO. (C) The mean mEPSC amplitude was significantly increased. (D) Whole cell currents were evoked by bath application of $25 \mathrm{nM} \mathrm{L-AMPA}$ during the indicated time period. A summary plot shows the enhanced response to L-AMPA of KO cells as compared to WT cells. Bar graphs show the average of values within the time periods 8-10 (bottom-left) and 1416 minutes (bottom-right). mEPSCs and AMPA currents were recorded in the presence of the sodium channel blocker Tetrodotoxin (TTX, $1 \mu \mathrm{M})$ and the AMPARdesensitization inhibitor Cyclothiazide (CTZ, $100 \mu \mathrm{M})$; numbers $(n)$ of cells analyzed (from >4 animals each) are shown at the bottom of the bar graphs; error bars 
Results

represent sem; all statistical analyses were carried out using the two-tailed Student's $t$-test; ${ }^{*}, p<0.05 ;{ }^{* *}, p<0.01$; ns, not significant. 


\section{V.2.5. Rasgrp1 KO Mice Show Increased PSD-95 Expression}

\section{V.2.5.1. Imaging of Synaptic Composition in the Rasgrp1 KO Mouse}

Electrophysiological experiments indicate a postsynaptic component of the abnormal transmission seen in Rasgrp1 KO mice. To obtain additional evidence that the postsynaptic, but not the presynaptic compartment is the major source of these changes, a detailed imaging study was performed to investigate the number and composition of synapses in the hippocampal CA1 region. Coronal brain sections of WT and Rasgrp1 KO mice were immunolabeled for specific pre- and postsynaptic marker proteins. These stainings resulted in punctuate signals, which were counted and analyzed with respect to their intensities and area.

Axons running through the CA1 stratum radiatum terminate at apical dendrites of CA1 pyramidal cells in the stratum radiatum itself, but also branch and target basal dendrites in the stratum oriens and the dendritic tufts of pyramidal neurons in the stratum lacunosum moleculare. These axons derive from neurons of the CA3 region, but also from neurons in the entorhinal cortex. The stratum lacunosum moleculare also receives input from the thalamus (Klausberger and Somogyi, 2008). In the field recordings described above, stimulus and recording electrode were placed in the stratum radiatum. To investigate, if other regions were affected by knock out of Rasgrp1, the laminae of the hippocampus were analyzed separately.

\section{V.2.5.1.1. Imaging of Excitatory Postsynapses}

First, immunolabelings for PSD-95 were analyzed. PSD-95 is highly enriched in the postsynapse of glutamatergic synapses and therefore is frequently used as a marker protein for this compartment. PSD-95 is the major postsynaptic scaffolding protein of the PSD (Cheng et al., 2006; Cho et al., 1992; Feng and Zhang, 2009) and was additionally reported to associate with the postsynaptic membrane itself via posttranslational palmitoylation (Craven et al., 1999).

Immunohistochemical studies detected PSD-95 throughout all layers of the hippocampus with highest levels of expression in the stratum oriens and the stratum radiatum (Chang et al., 2009; Hunt et al., 1996). This staining pattern was confirmed in immunolabelings of WT sections performed in the present study. Interestingly, higher levels of fluorescence intensity in all strata of Rasgrp1 KO sections were evident even from cursory comparison at the fluorescence binocular (Figure V.10 A). 
Quasi-confocal (grid-projection) images were taken from each lamina of the CA1 region and processed as described in chapter IV.4 (Figure V.10 B). Mean intensity of puncta did not differ significantly in all strata analyzed, but relative frequency distribution of values obtained from analyses of Rasgrp1 KO sections showed clear shifts to higher intensity values (Figure V.10 C). The pairs of values tested at several intervals showed statistically significant differences in the stratum oriens, stratum pyramidale and stratum radiatum (five value pairs tested each). Only the shift of intensity distributions for the PSD-95 puncta imaged in the stratum lacunosum moleculare was not significantly different between WT and KO at all of the intervals tested (Table V.4).

Table V.4: Intensities of PSD-95 puncta in the Rasgrp1 KO hippocampus

\begin{tabular}{|c|c|c|c|c|c|c|c|}
\hline \multicolumn{4}{|c|}{ stratum oriens } & \multicolumn{4}{|c|}{ stratum pyramidale } \\
\hline & WT & $\mathrm{KO}$ & \multirow{2}{*}{$\begin{array}{c}p \\
0.546\end{array}$} & \multicolumn{2}{|r|}{ WT } & $\mathrm{KO}$ & \multirow{2}{*}{$\begin{array}{c}p \\
0.342\end{array}$} \\
\hline mean & $14.18 \pm 2.70$ & $16.93 \pm 3.36$ & & mean & $12.39 \pm 1.85$ & $15.39 \pm 2.33$ & \\
\hline 20 & $0.120 \pm 0.027$ & $0.035 \pm 0.014$ & ${ }^{*} 0.018$ & 12 & $0.108 \pm 0.027$ & $0.017 \pm 0.011$ & ${ }^{* \star} 0.003$ \\
\hline 24 & $0.130 \pm 0.031$ & $0.072 \pm 0.017$ & 0.135 & 16 & $0.123 \pm 0.024$ & $0.042 \pm 0.012$ & ${ }^{* *} 0.007$ \\
\hline 28 & $0.112 \pm 0.026$ & $0.121 \pm 0.020$ & 0.801 & 20 & $0.129 \pm 0.023$ & $0.111 \pm 0.018$ & 0.352 \\
\hline 32 & $0.083 \pm 0.017$ & $0.137 \pm 0.025$ & 0.109 & 24 & $0.116 \pm 0.022$ & $0.160 \pm 0.027$ & 0.816 \\
\hline 36 & $0.059 \pm 0.013$ & $0.124 \pm 0.019$ & ${ }^{*} 0.016$ & 28 & $0.088 \pm 0.021$ & $0.171 \pm 0.037$ & 0.153 \\
\hline \multicolumn{4}{|c|}{ stratum radiatum } & \multicolumn{4}{|c|}{ stratum lacunosum moleculare } \\
\hline & WT & $\mathrm{KO}$ & $p$ & & WT & $\mathrm{KO}$ & $p$ \\
\hline mean & $14.11 \pm 2.54$ & $16.32 \pm 3.08$ & 0.436 & mean & $14.13 \pm 3.06$ & $14.76 \pm 2.21$ & 0.880 \\
\hline 20 & $0.153 \pm 0.033$ & $0.085 \pm 0.038$ & 0.211 & 20 & $0.144 \pm 0.024$ & $0.085 \pm 0.024$ & 0.105 \\
\hline 24 & $0.153 \pm 0.031$ & $0.127 \pm 0.041$ & 0.636 & 24 & $0.158 \pm 0.027$ & $0.105 \pm 0.021$ & 0.154 \\
\hline 28 & $0.111 \pm 0.021$ & $0.156 \pm 0.038$ & 0.336 & 28 & $0.132 \pm 0.027$ & $0.131 \pm 0.028$ & 0.970 \\
\hline 32 & $0.073 \pm 0.013$ & $0.142 \pm 0.027$ & ${ }^{*} 0.043$ & 32 & $0.091 \pm 0.024$ & $0.134 \pm 0.029$ & 0.288 \\
\hline 36 & $0.052 \pm 0.008$ & $0.110 \pm 0.018$ & ${ }^{*} 0.013$ & 36 & $0.063 \pm 0.019$ & $0.103 \pm 0.021$ & 0.188 \\
\hline
\end{tabular}

Datasets are organized in sections, headed by the name of the region investigated. The first row of each section contains the mean intensity values. Below, the relative frequency values at the indicated intervals are shown. $n, \mathrm{WT}=12$ sections; $n, \mathrm{KO}=11$ sections; all values are given as means \pm sem. Statistical analyses were carried out using the two-tailed Students $t$-test.

Similarly to the relatively low PSD-95 immunofluorescence of the stratum pyramidale, the number of PSD-95 puncta per $100 \mu \mathrm{m}^{2}$ was also lowest in this lamina when compared to other strata (Figure V.10 D). Comparison of counts revealed that the mean numbers of PSD-95 puncta were significantly increased in every region of KO hippocampi (Figure V.10 D, Table V.5). The mean area of puncta in Rasgrp1 KO mice was comparable to that of WT puncta in every lamina, except for the stratum pyramidale (Figure V.10 E), where a slight but significant difference of $13 \%$ was observed (Table V.5). 
Table V.5: Numbers and areas of PSD-95 puncta in the Rasgrp1 KO hippocampus

\begin{tabular}{|c|c|c|c|c|c|c|c|}
\hline \multicolumn{5}{|c|}{ stratum oriens } & \multicolumn{4}{c|}{ stratum pyramidale } \\
\hline mean & WT & $\mathrm{KO}$ & $p$ & mean & WT & KO & $p$ \\
\hline number & $20.91 \pm 1.60$ & $28.86 \pm 0.98$ & ${ }^{* *} 0.001$ & number & $16.08 \pm 1.06$ & $21.07 \pm 0.80$ & ${ }^{* *} 0.002$ \\
\hline area & $0.225 \pm 0.006$ & $0.231 \pm 0.003$ & 0.958 & area & $0.222 \pm 0.008$ & $0.255 \pm 0.010$ & ${ }^{*} 0.023$ \\
\hline \multicolumn{7}{|c|}{ stratum radiatum } & \multicolumn{5}{c|}{ stratum lacunosum moleculare } \\
\hline mean & $\mathrm{WT}$ & $\mathrm{KO}$ & $p$ & mean & WT & KO & $p$ \\
\hline number & $18.12 \pm 1.19$ & $23.35 \pm 1.23$ & ${ }^{*} 0.02$ & number & $23.07 \pm 1.07$ & $27.91 \pm 1.89$ & ${ }^{*} 0.03$ \\
\hline area & $0.231 \pm 0.007$ & $0.235 \pm 0.005$ & 0.613 & area & $0.231 \pm 0.007$ & $0.229 \pm 0.006$ & 0.172 \\
\hline
\end{tabular}

Datasets are organized in sections, headed by the name of the region investigated. The first row of each section contains the mean numbers. The row below displays the mean areas $\left[\mu \mathrm{m}^{2}\right] . n, \mathrm{WT}=12$; $n, \mathrm{KO}=11$; all values are given as means $\pm s e m$. Statistical analyses were carried out using the twotailed Students $t$-test.

The increased intensity and number of PSD-95 puncta in Rasgrp1 KOs substantiates the hypothesis of postsynaptic strengthening in the Rasgrp1 KO hippocampus. The regions observed by this method theoretically cover the whole postsynaptic morphological structure of pyramidal neurons. The stratum oriens contains basal dendrites, the stratum pyramidale contains the somata, the stratum radiatum contains the apical dendrites, and the stratum lacunosum moleculare contains the dendritic tufts (Spruston, 2008). Therefore, the effect of Rasgrp1 KO on PSD-95 seems to be general, since all of these regions show higher numbers of PSD-95 puncta and increased intensities. A general mechanism leading to these observations would be an increased expression of PSD95. 
A

B

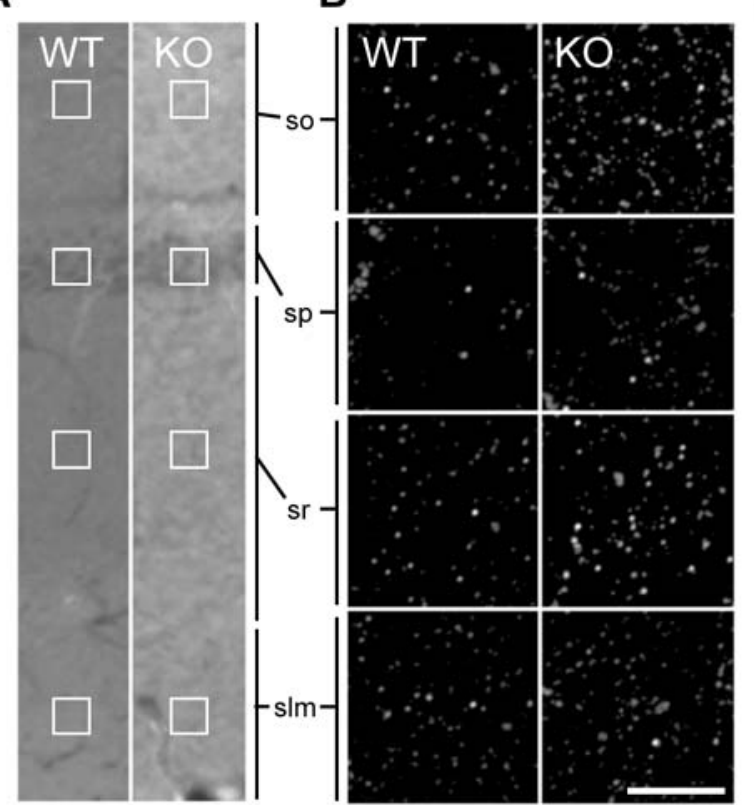

C
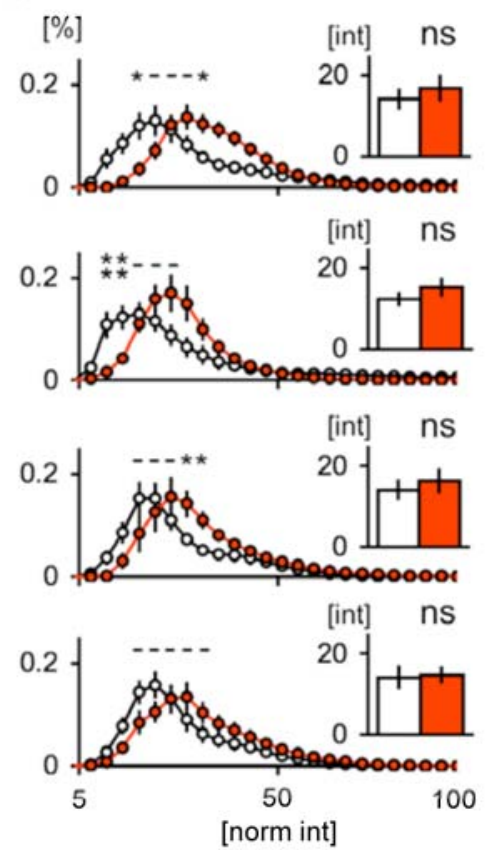

D

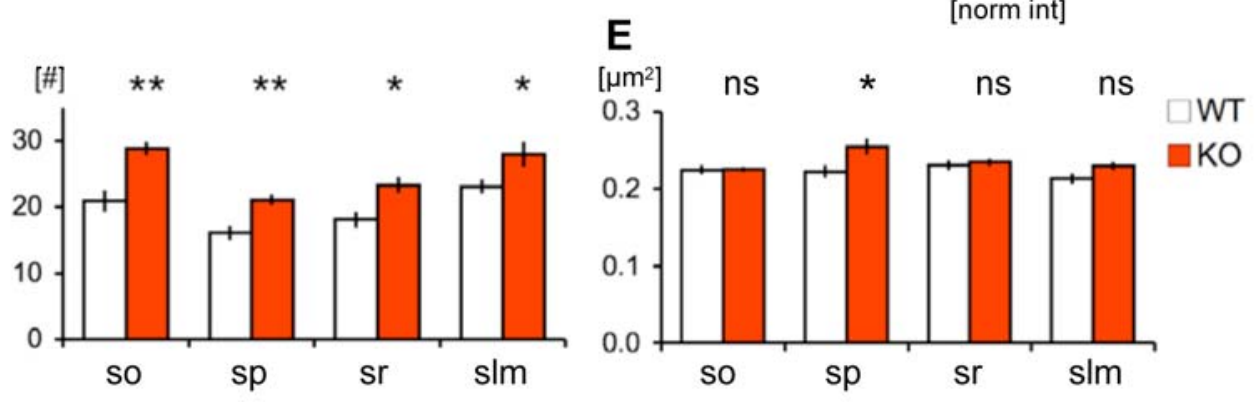

Figure V.10: Imaging of excitatory postsynapses in CA1 of Rasgrp1 KO mice

Excitatory postsynapses were specifically labeled by immunostaining for PSD-95. The number and intensity of PSD-95 were significantly increased in Rasgrp1 knockout (KO) mice (3-5 weeks old).

(A) Sample cross-sections through the CA1 strata of WT and KO mice (boxes = 20x20 $\mu \mathrm{m}$ ) stained for PSD-95. (B) Regions of interest (ROIs) were selected from 12 WT and $11 \mathrm{KO}$ coronal sections (>3 animals each) and processed. PSD-95 puncta, which had areas between 0.06 and $2.5 \mu \mathrm{m}^{2}$ and circularities between 0.6 and 1, were counted and intensities measured. Scale bar $=10 \mu \mathrm{m}$. (C) In KO sections, the relative frequency distribution of normalized intensities shows a significant shift to higher intensities for stratum oriens (so), stratum pyramidale (sp) and stratum radiatum (sr), but not for stratum lacunosum moleculare (slm). The five intervals around the intersection of WT and KO distributions were statistically analyzed. Bar graphs show the mean intensity of PSD-95 puncta, which were not significantly different in any region. (D) The mean puncta count per $100 \mu \mathrm{m}^{2}$ (\#) was significantly increased in every region analyzed. (E) The mean area of puncta was unchanged, 
Results

except for a slight, but significant increase in the stratum pyramidale of Rasgrp1 KO animals. Error bars represent sem; all statistical analyses were carried out using the two-tailed Student's $t$-test; ${ }^{*}, p<0.05 ;{ }^{* *}, p<0.01$; ns, - , not significant. 


\section{V.2.5.1.2. Imaging of Presynapses}

Stronger intensities and increased number of PSD-95 puncta in Rasgrp1 KO animals could result from two phenomena. First, increased PSD-95 expression in Rasgrp1 KO mice could result in a higher number and stronger labeling of postsynapses. Secondly, increased PSD-95 expression could result in stronger labeling of the postsynapse only. The increased count would then arise from a higher probability of detection because PSD-95 puncta were more intensely labeled. mEPSC frequency was unchanged in the Rasgrp1 $\mathrm{KO}$, indicating that the number of functional synapses is unchanged. In that case, the number and strength of presynapses in the CA1 region of Rasgrp1 KO mice should be normal.

Synapsins are proteins found in most synapses in the brain. They tether synaptic vesicles to the cytoskeleton and to each other (Cesca et al., 2010; Evergren et al., 2007). Several isoforms are produced from the three genes (Synapsin1-3), of which the Synapsin 1 and 2 isoforms are found exclusively at presynaptic terminals, whereas Synapsin3 can also be detected in the somata of neurons (Cesca et al., 2010; Evergren et al., 2007; Ferreira et al., 2000). An antibody recognizing Synapsin 1 and 2 therefore seemed appropriate to specifically label presynapses in the present study.

As described in other studies, highest Synapsin1/2 immunoreactivity was detected in the stratum radiatum, followed by the stratum oriens. The stratum lacunosum was less intensely stained, and in the stratum pyramidale only very few immunoreactive puncta were detected (Figure V.11 A, B). Presynaptic intensity profiles as well as mean intensities were not significantly different between Rasgrp1 KO and WT mice (Figure V.11 C; Table V.6). 
Table V.6: Intensities of Synapsin1/2 puncta in the Rasgrp1 KO hippocampus

\begin{tabular}{|c|c|c|c|c|c|c|c|}
\hline \multicolumn{4}{|c|}{ stratum oriens } & \multicolumn{4}{|c|}{ stratum pyramidale } \\
\hline & WT & $\mathrm{KO}$ & $p$ & & WT & $\mathrm{KO}$ & $p$ \\
\hline mean & $18.36 \pm 2.44$ & $18.06 \pm 1.61$ & 0.923 & mean & $17.61 \pm 1.96$ & $16.05 \pm 1.46$ & 0.549 \\
\hline 16 & $0.111 \pm 0.026$ & $0.121 \pm 0.019$ & 0.779 & 16 & $0.111 \pm 0.018$ & $0.208 \pm 0.075$ & 0.245 \\
\hline 20 & $0.130 \pm 0.029$ & $0.189 \pm 0.032$ & 0.199 & 20 & $0.154 \pm 0.023$ & $0.225 \pm 0.058$ & 0.288 \\
\hline 24 & $0.156 \pm 0.021$ & $0.171 \pm 0.025$ & 0.668 & 24 & $0.159 \pm 0.022$ & $0.161 \pm 0.028$ & 0.964 \\
\hline 28 & $0.137 \pm 0.021$ & $0.140 \pm 0.017$ & 0.928 & 28 & $0.139 \pm 0.017$ & $0.111 \pm 0.029$ & 0.428 \\
\hline 32 & $0.110 \pm 0.023$ & $0.120 \pm 0.022$ & 0.767 & 32 & $0.106 \pm 0.016$ & $0.069 \pm 0.018$ & 0.148 \\
\hline \multicolumn{4}{|c|}{ stratum radiatum } & \multicolumn{4}{|c|}{ stratum lacunosum moleculare } \\
\hline & WT & $\mathrm{KO}$ & $p$ & & WT & $\mathrm{KO}$ & $p$ \\
\hline mean & $22.96 \pm 3.01$ & $19.69 \pm 1.28$ & 0.365 & mean & $13.47 \pm 1.36$ & $12.26 \pm 0.831$ & 0.478 \\
\hline 32 & $0.106 \pm 0.016$ & $0.069 \pm 0.018$ & 0.096 & 12 & $0.155 \pm 0.033$ & $0.231 \pm 0.038$ & 0.156 \\
\hline 36 & $0.069 \pm 0.011$ & $0.041 \pm 0.010$ & 0.185 & 16 & $0.223 \pm 0.038$ & $0.289 \pm 0.051$ & 0.330 \\
\hline 40 & $0.036 \pm 0.006$ & $0.022 \pm 0.006$ & 0.832 & 20 & $0.206 \pm 0.038$ & $0.187 \pm 0.035$ & 0.722 \\
\hline 44 & $0.020 \pm 0.003$ & $0.012 \pm 0.003$ & 0.317 & 24 & $0.132 \pm 0.019$ & $0.104 \pm 0.020$ & 0.338 \\
\hline 48 & $0.013 \pm 0.003$ & $0.007 \pm 0.002$ & 0.102 & 28 & $0.065 \pm 0.012$ & $0.044 \pm 0.010$ & 0.188 \\
\hline
\end{tabular}

Datasets are organized in sections, headed by the name of region investigated. The first row of each section contains the mean intensity values. Below, the relative frequency values at the indicated intervals are shown. $n, \mathrm{WT}=15 \mathrm{~s} ; n, \mathrm{KO}=14$; all values are given as means \pm sem. Statistical analyses were carried out using the two-tailed Students $t$-test.

Interestingly, mean puncta counts in the stratum oriens and the stratum radiatum resulted in small, but significant differences between WT and Rasgrp1 KO mice (Figure V.11 D). In these regions, mean numbers of Synapsin $1 / 2$ positive presynapses were decreased in KOs by $\sim 6-7 \%$. Mean numbers in the stratum pyramidale and the stratum lacunosum moleculare were not significantly different (Table V.7) The mean area of puncta was significantly decreased in the stratum pyramidale, whereas they appeared normal in the other strata (Figure V.11 E; Table v.7).

Although a small decrease in mean counts and sizes of Synapsin $1 / 2$ positive puncta was detected in some regions, this effect was not as prominent as the increase in intensities and numbers of PSD-95 puncta. However, it cannot be ruled out that the presynapse is slightly affected by knock-out of Rasgrp1. 
Table V.7: Numbers and areas of Synapsin1/2 puncta in the Rasgrp1 KO hippocampus

\begin{tabular}{|c|c|c|c|c|c|c|c|}
\hline \multicolumn{4}{|c|}{ stratum oriens } & \multicolumn{4}{c|}{ stratum pyramidale } \\
\hline mean & WT & $\mathrm{KO}$ & $p$ & mean & WT & $\mathrm{KO}$ & $p$ \\
\hline number & $47.45 \pm 0.69$ & $43.76 \pm 1.27$ & ${ }^{*} 0.041$ & number & $15.85 \pm 0.51$ & $16.76 \pm 1.10$ & 0.425 \\
\hline area & $0.29 \pm 0.006$ & $0.29 \pm 0.005$ & 0.613 & area & $0.39 \pm 0.009$ & $0.35 \pm 0.011$ & ${ }^{* *} 0.009$ \\
\hline \multicolumn{3}{|c|}{ stratum radiatum } & \multicolumn{4}{c|}{ stratum lacunosum moleculare } \\
\hline mean & WT & $\mathrm{KO}$ & $p$ & mean & WT & KO & $p$ \\
\hline number & $51.88 \pm 0.61$ & $49.44 \pm 1.08$ & ${ }^{*} 0.027$ & number & $39.38 \pm 1.89$ & $36.30 \pm 1.49$ & 0.424 \\
\hline area & $0.30 \pm 0.005$ & $0.30 \pm 0.006$ & 0.5 & area & $0.27 \pm 0.007$ & $0.26 \pm 0.005$ & 0.62 \\
\hline
\end{tabular}

Datasets are organized in sections, headed by the name of region investigated. The first row of each section contains the mean numbers. The row below displays the mean areas $\left[\mu \mathrm{m}^{2}\right] . n, \mathrm{WT}=15 ; n, \mathrm{KO}$ $=14$. All values are given as mean \pm sem. Statistical analyses were carried out using the two-tailed Students $t$-test.

A

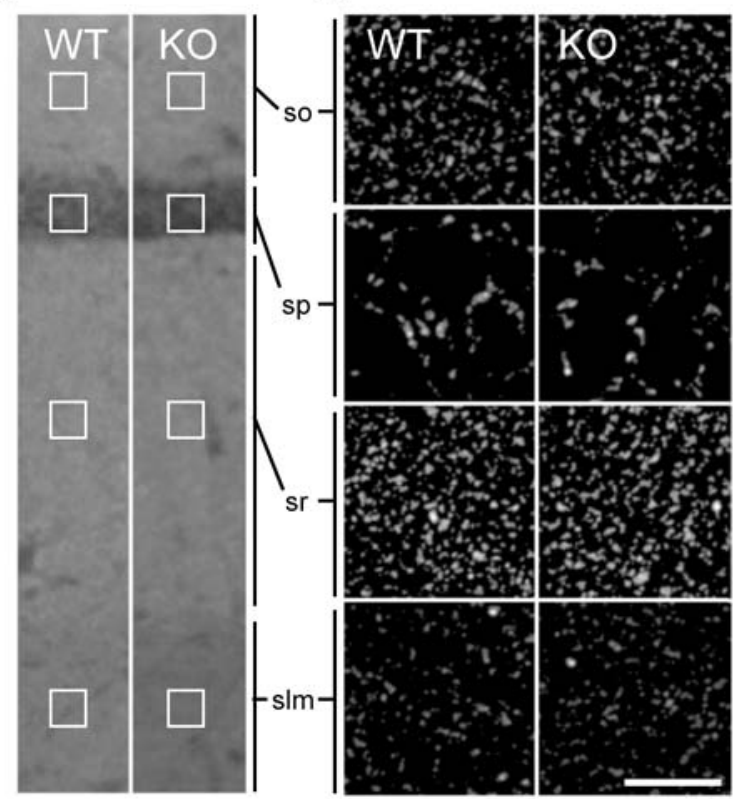

C
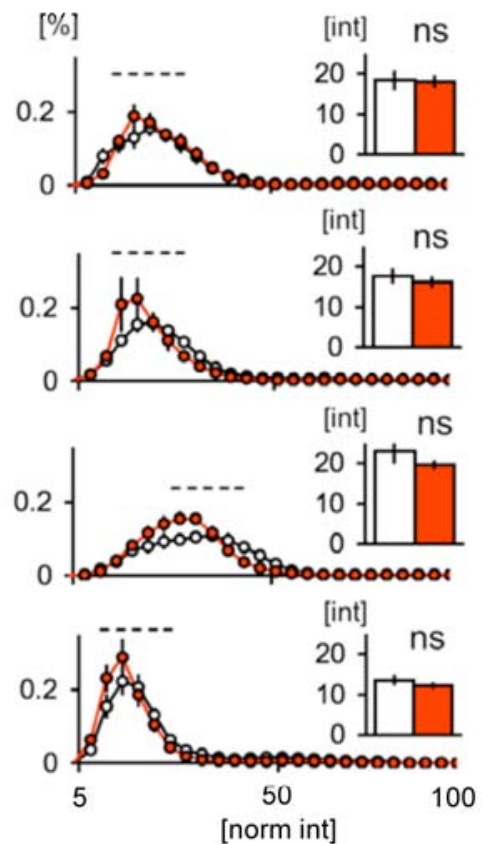

D
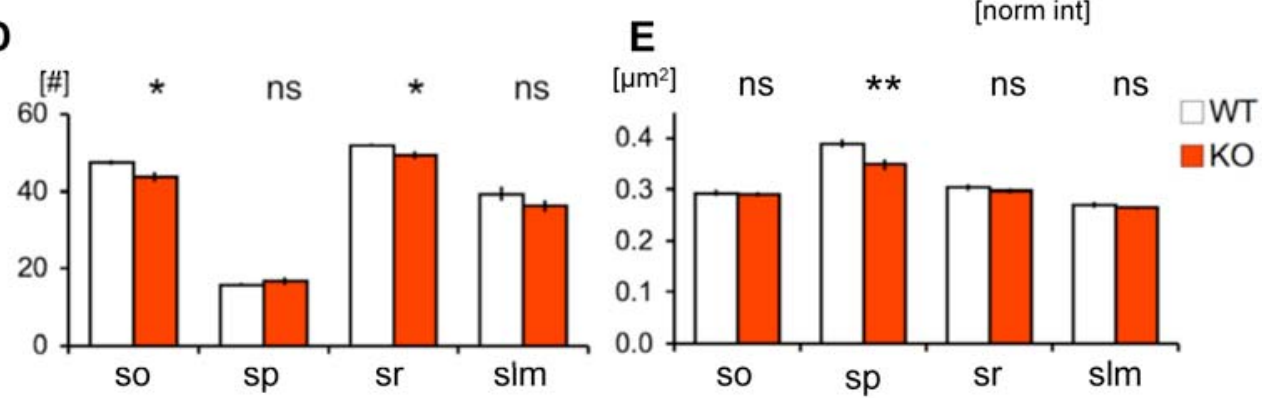

Figure V.11: Imaging of presynapses in the CA1 region of Rasgrp1 KO mice

Excitatory and inhibitory presynapses were labeled by immunostaining for Synapsin1/2 The intensity of puncta was unchanged and numbers differed only slightly between Rasgrp1 knock-out (KO) and WT mice (3-5 weeks). 
(A) Sample cross-sections through the CA1 strata of WT and KO mice (boxes = $20 \times 20 \mu \mathrm{m}$ ) stained for Synapsin1/2. (B) Regions of interest (ROls) were selected from $15 \mathrm{WT}$ and $14 \mathrm{KO}$ coronal sections ( $>3$ animals each) and processed. Synapsin1/2 puncta, which had areas between 0.09 and $2.5 \mu \mathrm{m}^{2}$ and circularities between 0.5 and 1, were counted and intensities measured. Scale bar $=10 \mu \mathrm{m}$. (C) Relative frequency distributions of normalized intensities show no significant difference in any region. The five intervals around the intersection of WT and KO distributions were statistically analyzed. Bar graphs show the mean intensities of Synapsin $1 / 2$ puncta, which were not significantly different in any region. (D) The mean puncta count per $100 \mu^{2}$ (\#) was slightly, but significantly reduced in stratum oriens (so) and stratum radiatum (sr) of KOs. (D) Mean areas of puncta were unchanged, except for a small, but significant decrease in the stratum pyramidale of Rasgrp1 $\mathrm{KO}$ animals.

so, stratum oriens; sp, stratum pyramidale; sr, stratum radiatum; slm, stratum lacunosum moleculare. Error bars represent sem; all statistical analyses were carried out using the two-tailed Student's $t$-test; ${ }^{*}, p<0.05 ;{ }^{* *}, p<0.01$; ns, -, not significant. 


\section{V.2.5.1.3. Imaging of Excitatory Presynapses}

Synapsin $1 / 2$ is found in both excitatory and inhibitory presynaptic terminals. In order to control the equilibrium that normally exists between excitatory and inhibitory synapses to maintain synaptic integrity, specific immunolabelings for excitatory and inhibitory presynapses were analyzed next.

VGLUT1 is the main vesicular glutamate transporter at excitatory presynapses in the hippocampus (Herzog et al., 2006). It is nearly absent from the stratum pyramidale as the vast majority of excitatory inputs arrive in the other strata. VGLUT1 localizes primarily to the stratum radiatum and the stratum oriens (Bellocchio et al., 1998). This pattern is also seen in Figure V.12 A and B. In all laminae of Rasgrp1 KO hippocampi, numbers and intensities of VGLUT1 positive puncta were similar to WT values (Figure V.12 C, D; Table V.8). The mean area of puncta was slightly decreased in all strata, but this difference was only statistically significant in the stratum oriens (Figure V.12 E; Table V.9)

These results show, that excitatory presynapses in general and the Glutamate transport machinery at synaptic vesicles in particular, are likely to be unaffected by loss of Rasgrp1.

Table V.8: Intensities of VGLUT1 puncta in the Rasgrp1 KO hippocampus

\begin{tabular}{|c|c|c|c|c|c|c|c|}
\hline \multicolumn{4}{|c|}{ stratum oriens } & \multicolumn{4}{c|}{ stratum pyramidale } \\
\hline & WT & $\mathrm{KO}$ & $p$ & & WT & KO & $p$ \\
\hline mean & $23.36 \pm 0.58$ & $21.46 \pm 1.29$ & 0.223 & mean & $16.60 \pm 0.99$ & $17.00 \pm 1.08$ & 0.839 \\
\hline $\mathbf{3 6}$ & $0.108 \pm 0.018$ & $0.108 \pm 0.018$ & 36 & $\mathbf{1 2}$ & $0.074 \pm 0.019$ & $0.103 \pm 0.021$ & 0.245 \\
\hline $\mathbf{4 0}$ & $0.112 \pm 0.016$ & $0.123 \pm 0.021$ & 40 & $\mathbf{1 6}$ & $0.108 \pm 0.030$ & $0.120 \pm 0.034$ & 0.288 \\
\hline $\mathbf{4 4}$ & $0.113 \pm 0.012$ & $0.113 \pm 0.021$ & 44 & $\mathbf{2 0}$ & $0.129 \pm 0.028$ & $0.111 \pm 0.034$ & 0.964 \\
\hline $\mathbf{4 8}$ & $0.110 \pm 0.009$ & $0.095 \pm 0.022$ & 48 & $\mathbf{2 4}$ & $0.142 \pm 0.031$ & $0.102 \pm 0.017$ & 0.428 \\
\hline $\mathbf{5 2}$ & $0.090 \pm 0.011$ & $0.075 \pm 0.024$ & 52 & $\mathbf{2 8}$ & $0.158 \pm 0.039$ & $0.100 \pm 0.015$ & 0.148 \\
\hline \multicolumn{7}{|c|}{ stratum radiatum } & \multicolumn{5}{|c|}{ stratum lacunosum moleculare } \\
\hline mean & $24.43 \pm 0.65$ & $23,64 \pm 0.99$ & 0.398 & mean & $16.34 \pm 0.70$ & $16.73 \pm 0.76$ & 0.5 \\
\hline $\mathbf{3 6}$ & $0.098 \pm 0.017$ & $0.100 \pm 0.012$ & 0.950 & $\mathbf{2 0}$ & $0.129 \pm 0.028$ & $0.111 \pm 0.034$ & 0.156 \\
\hline $\mathbf{4 0}$ & $0.111 \pm 0.016$ & $0.135 \pm 0.020$ & 0.395 & $\mathbf{2 4}$ & $0.142 \pm 0.031$ & $0.102 \pm 0.017$ & 0.330 \\
\hline $\mathbf{4 4}$ & $0.124 \pm 0.019$ & $0.143 \pm 0.032$ & 0.633 & $\mathbf{2 8}$ & $0.158 \pm 0.039$ & $0.100 \pm 0.015$ & 0.722 \\
\hline $\mathbf{4 8}$ & $0.124 \pm 0.021$ & $0.127 \pm 0.030$ & 0.938 & $\mathbf{3 2}$ & $0.126 \pm 0.032$ & $0.089 \pm 0.017$ & 0.338 \\
\hline $\mathbf{5 2}$ & $0.103 \pm 0.017$ & $0.107 \pm 0.025$ & 0.909 & $\mathbf{3 6}$ & $0.084 \pm 0.022$ & $0.084 \pm 0.016$ & 0.188 \\
\hline
\end{tabular}

Datasets are organized in sections, headed by the name of region investigated. The first row of each section contains the mean intensity values. Below, the relative frequency values at the indicated intervals are shown. $n, \mathrm{WT}=12 ; n, \mathrm{KO}=9$; all values are given as mean $\pm s e m$. Statistical analyses were carried out using the two-tailed Students $t$-test. 
Table V.9: Numbers and areas of VGLUT1 puncta in the Rasgrp1 KO hippocampus

\begin{tabular}{|c|c|c|c|c|c|c|c|}
\hline \multicolumn{4}{|c|}{ stratum oriens } & \multicolumn{4}{c|}{ stratum pyramidale } \\
\hline mean & WT & $\mathrm{KO}$ & $p$ & mean & WT & KO & $p$ \\
\hline number & $34.28 \pm 1.30$ & $36.51 \pm 1.71$ & 0.09 & number & $2.82 \pm 0.20$ & $3.92 \pm 0.86$ & 0.217 \\
\hline area & $0.43 \pm 0.005$ & $0.38 \pm 0.015$ & ${ }^{* *} 0.008$ & area & $0.4 \pm 0.009$ & $0.37 \pm 0.021$ & 0.253 \\
\hline \multicolumn{5}{|c|}{ stratum radiatum } & \multicolumn{4}{c|}{ stratum lacunosum moleculare } \\
\hline mean & WT & $\mathrm{KO}$ & $p$ & mean & WT & KO & $p$ \\
\hline number & $35.23 \pm 1.61$ & $37.29 \pm 1.46$ & 0.06 & number & $31.44 \pm 0.98$ & $33.12 \pm 1.22$ & 0.183 \\
\hline area & $0.42 \pm 0.01$ & $0.40 \pm 0.017$ & 0.407 & area & $0.27 \pm 0.007$ & $0.35 \pm 0.012$ & 0.49 \\
\hline
\end{tabular}

Datasets are organized in sections, headed by the name of region investigated. The first row of each section contains the mean numbers. The row below displays the mean areas $\left[\mu \mathrm{m}^{2}\right] . n, \mathrm{WT}=12 ; n, \mathrm{KO}$ = 9; all values are given as mean $\pm s e m$; statistical analyses were carried out using the two-tailed Students $t$-test.

A

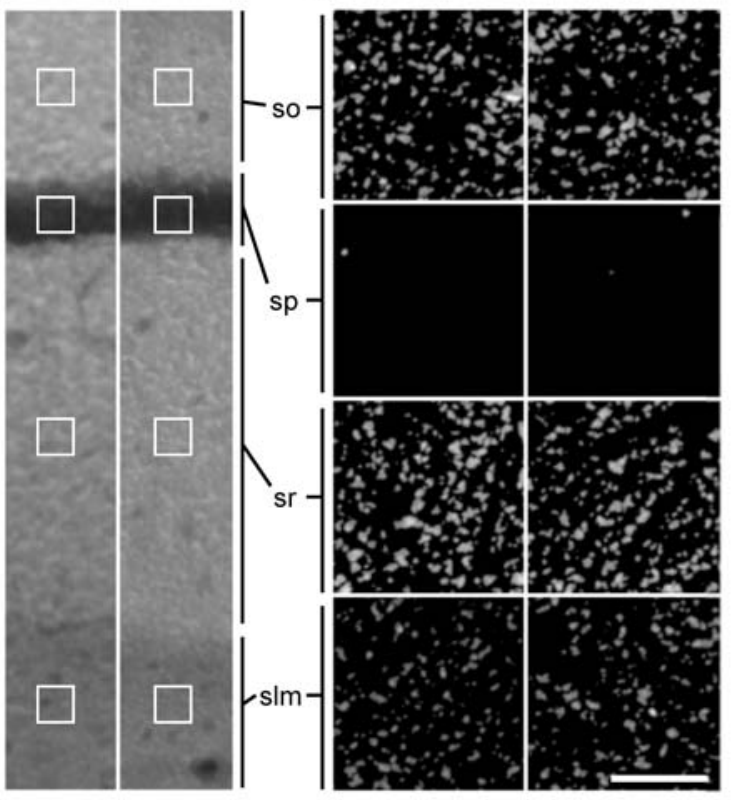

C

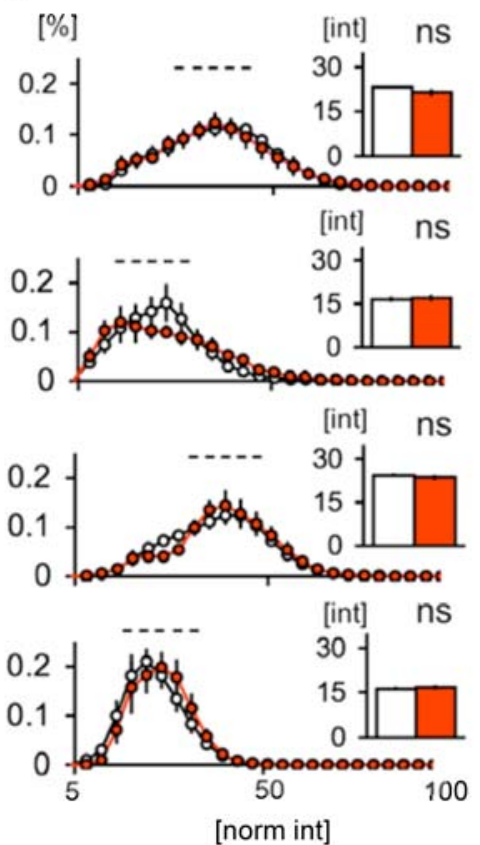

D

\section{E}
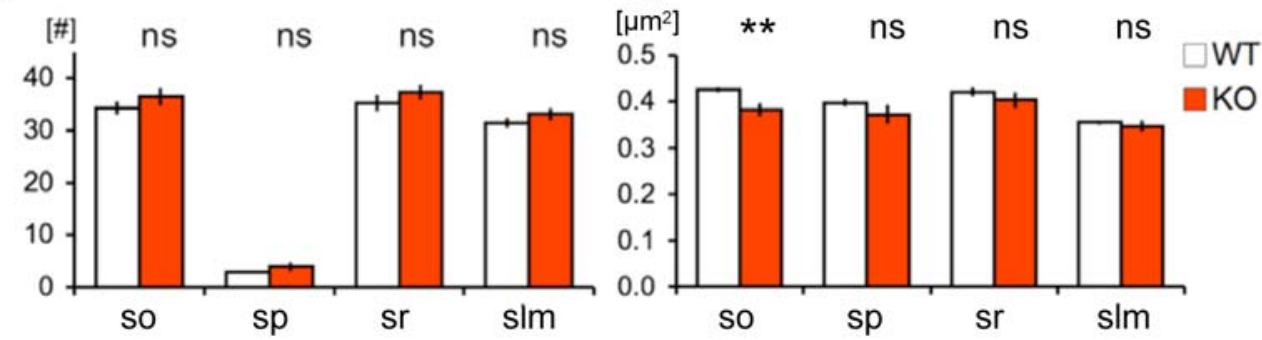

Figure V.12: Imaging of excitatory presynapses in the CA1 region of Rasgrp1 KO mice

Excitatory presynapses were labeled by immunostaining for VGLUT1. The intensity and number of puncta were not changed in Rasgrp1 knock-out (KO) mice (3-5 weeks). 
(A) Sample cross-sections through the CA1 strata of WT and KO mice (boxes = 20x20 $\mu \mathrm{m}$ ) stained for VGLUT1. (B) Regions of interest (ROIs) were selected from 12 WT and $9 \mathrm{KO}$ coronal sections ( $>3$ animals each) and processed. VGLUT1 puncta, which had areas between 0.09 and $2.5 \mu \mathrm{m}^{2}$ and circularities between 0.5 and 1 , were counted and intensities measured. Scale bar $=10 \mu \mathrm{m}$. (C) The relative frequency distributions of normalized intensities show no significant difference in any region. The five intervals around the intersection of frequency distribution or, alternatively, the common peak of WT and KO histograms were statistically analyzed. Bar graphs show the mean intensity of VGLUT1 puncta, which were equally not significantly different in any region. (D) The mean puncta count per $100 \mu \mathrm{m}^{2}$ (\#) was not different in any stratum. (E) The mean area of puncta was slightly changed in the stratum oriens (so) of Rasgrp1 KO mice, but unaltered in other strata.

so, stratum oriens; sp, stratum pyramidale; sr, stratum radiatum; sIm, stratum lacunosum moleculare. Error bars represent sem; all statistical analyses were carried out using the two-tailed Students $t$-test; ${ }^{* *}, p<0.01 ; n s,-$, not significant. 


\section{V.2.5.1.4. Imaging of Inhibitory Presynapses}

VGAT is a vesicular transporter for GABA and is expressed throughout the CA1 region. It is concentrated in presynaptic termini around the somata of pyramidal neurons, i.e. in the stratum pyramidale (Chaudhry et al., 1998). In the other strata, it is less abundant (Figure V.13 A, B). In Rasgrp1 KO samples, neither the intensities nor the areas or numbers of VGAT puncta showed any significant difference as compared to WT values (Table V.10; Table V.11).

Table V.10: Intensities of VGAT puncta in the Rasgrp1 KO hippocampus

\begin{tabular}{|c|c|c|c|c|c|c|c|}
\hline \multicolumn{4}{|c|}{ stratum oriens } & \multicolumn{4}{|c|}{ stratum pyramidale } \\
\hline & WT & $\mathrm{KO}$ & $p$ & & WT & $\mathrm{KO}$ & $p$ \\
\hline mean & $19.02 \pm 2.08$ & $15.37 \pm 1.54$ & 0.182 & mean & $15.37 \pm 1.54$ & $18.07 \pm 0.98$ & 0.798 \\
\hline 20 & $0.106 \pm 0.018$ & $0.170 \pm 0.037$ & 0.170 & 20 & $0.090 \pm 0.022$ & $0.129 \pm 0.032$ & 0.357 \\
\hline 24 & $0.115 \pm 0.018$ & $0.161 \pm 0.047$ & 0.402 & 24 & $0.132 \pm 0.032$ & $0.159 \pm 0.030$ & 0.575 \\
\hline 28 & $0.129 \pm 0.018$ & $0.125 \pm 0.039$ & 0.933 & 28 & $0.141 \pm 0.026$ & $0.164 \pm 0.022$ & 0.519 \\
\hline 32 & $0.111 \pm 0.023$ & $0.094 \pm 0.036$ & 0.710 & 32 & $0.123 \pm 0.021$ & $0.161 \pm 0.023$ & 0.266 \\
\hline 36 & $0.088 \pm 0.033$ & $0.071 \pm 0.031$ & 0.723 & 36 & $0.102 \pm 0.022$ & $0.122 \pm 0.024$ & 0.554 \\
\hline \multicolumn{4}{|c|}{ stratum radiatum } & \multicolumn{4}{|c|}{ stratum lacunosum moleculare } \\
\hline & WT & $\mathrm{KO}$ & $p$ & & WT & $\mathrm{KO}$ & $p$ \\
\hline mean & $17.24 \pm 1.81$ & $15.08 \pm 1.30$ & 0.52 & mean & $14.75 \pm 0.93$ & $13.90 \pm 0.54$ & 0.736 \\
\hline 16 & $0.109 \pm 0.026$ & $0.161 \pm 0.044$ & 0.464 & 12 & $0.048 \pm 0.014$ & $0.106 \pm 0.031$ & 0.156 \\
\hline 20 & $0.102 \pm 0.020$ & $0.142 \pm 0.032$ & 0.650 & 16 & $0.161 \pm 0.033$ & $0.171 \pm 0.051$ & 0.330 \\
\hline 24 & $0.106 \pm 0.022$ & $0.115 \pm 0.017$ & 0.870 & 20 & $0.217 \pm 0.032$ & $0.196 \pm 0.030$ & 0.722 \\
\hline 28 & $0.126 \pm 0.030$ & $0.101 \pm 0.025$ & 0.646 & 24 & $0.157 \pm 0.022$ & $0.211 \pm 0.020$ & 0.338 \\
\hline 32 & $0.114 \pm 0.032$ & $0.100 \pm 0.043$ & 0.827 & 28 & $0.111 \pm 0.028$ & $0.170 \pm 0.030$ & 0.188 \\
\hline
\end{tabular}

Datasets are organized in sections, headed by the name of region investigated. The first row of each section contains the mean intensity values. Below, the relative frequency values at the indicated intervals are shown. $n, \mathrm{WT}=12 ; n, \mathrm{KO}=9$; all values are given as mean \pm sem; statistical analyses were carried out using the two-tailed Students $t$-test.

Table V.11: Numbers and areas of VGAT puncta in the Rasgrp1 KO hippocampus

\begin{tabular}{|c|c|c|c|c|c|c|c|}
\hline \multicolumn{4}{|c|}{ stratum oriens } & \multicolumn{4}{c|}{ stratum pyramidale } \\
\hline mean & WT & KO & $p$ & mean & WT & KO & $p$ \\
\hline number & $21.12 \pm 1.30$ & $22.08 \pm 3.26$ & 0.315 & number & $14.44 \pm 0.69$ & $15.24 \pm 0.95$ & 0.794 \\
\hline area & $0.32 \pm 0.009$ & $0.33 \pm 0.008$ & 0.799 & area & $0.40 \pm 0.009$ & $0.42 \pm 0.012$ & 0.156 \\
\hline \multicolumn{7}{|c|}{ stratum radiatum } & \multicolumn{5}{|c|}{ stratum lacunosum moleculare } \\
\hline mean & WT & KO & $p$ & mean & WT & KO & $p$ \\
\hline number & $24.41 \pm 3.15$ & $26.03 \pm 3.67$ & 0.377 & number & $27.29 \pm 1.46$ & $30.75 \pm 2.20$ & 0.608 \\
\hline area & $0.31 \pm 0.006$ & $0.32 \pm 0.009$ & 0.958 & area & $0.29 \pm 0.005$ & $0.30 \pm 0.005$ & 0.832 \\
\hline
\end{tabular}

Datasets are organized in sections, headed by the name of region investigated. The first row of each section contains the mean numbers. The row below displays the mean areas $\left[\mu \mathrm{m}^{2}\right] . n, \mathrm{WT}=12 ; n, \mathrm{KO}$ = 9; all values are given as means \pm sem; statistical analyses were carried out using the two-tailed Students $t$-test. 
The analyses of VGAT positive puncta indicate, that Rasgrp1 does not have an effect on inhibitory presynapses. Along with the other data obtained in this imaging study, this supports the view of a predominant role of Rasgrp1 in postsynaptic development and function. Moreover these data highlight a role of PSD-95 in the regulation of synaptic transmission by Rasgrp1.

A

B

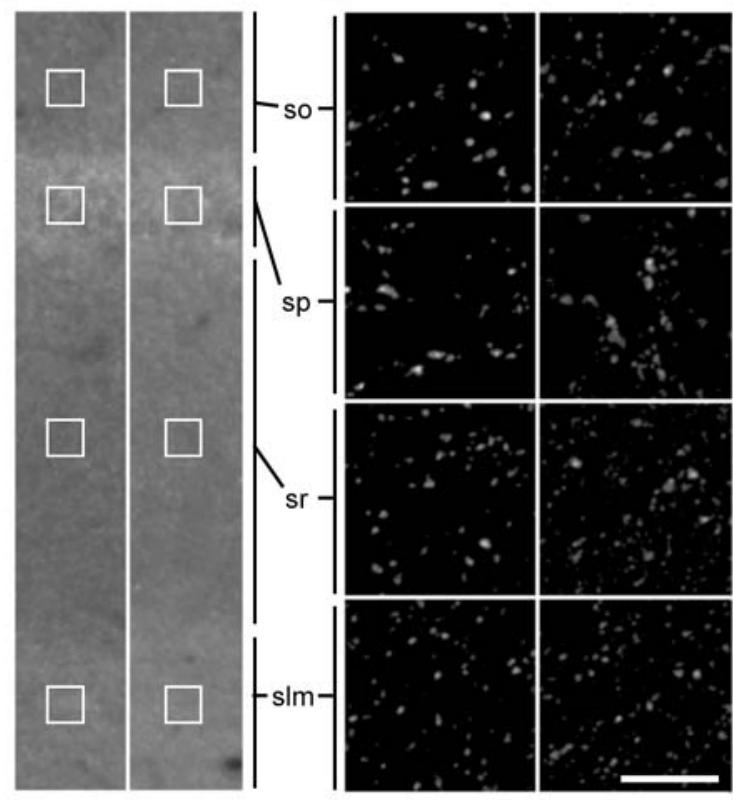

C
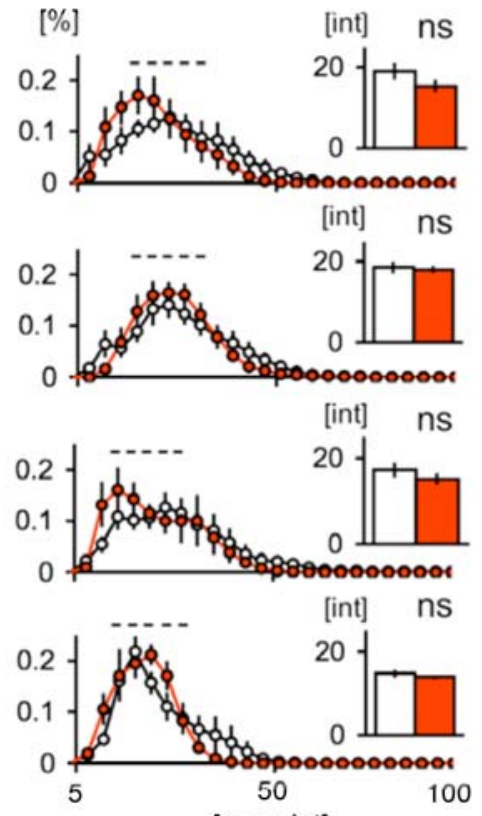

D

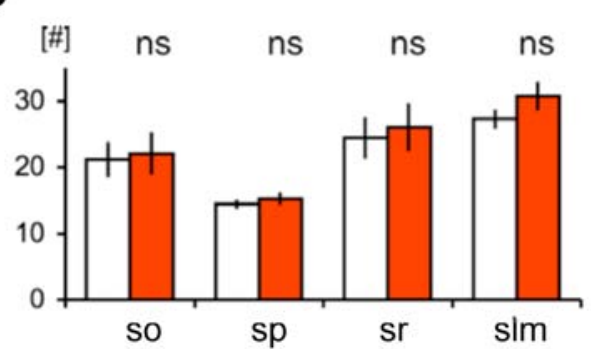

E

[norm int]

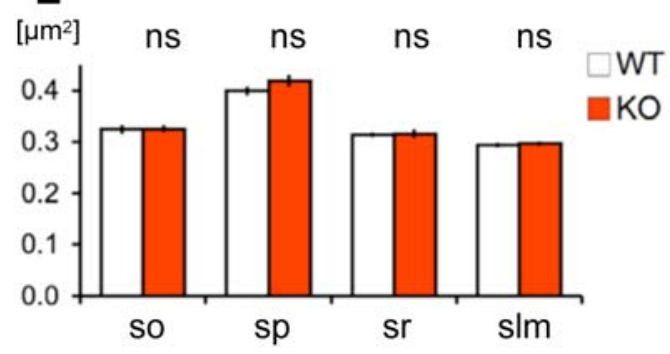

Figure V.13: Imaging of inhibitory presynapses in the CA1 region of Rasgrp1 KO mice

Inhibitory presynapses were labeled by immunostaining for VGAT. The intensity and number of puncta were not changed in Rasgrp1 knock-out (KO) mice (3-5 weeks).

(A) Sample cross-sections through the CA1 strata of WT and KO mice (boxes = 20x20 $\mu \mathrm{m}$ ) stained for VGAT. (B) Regions of interest (ROls) were selected from 12 WT and $9 \mathrm{KO}$ coronal sections ( $>3$ animals each) and processed. VGAT puncta, which had areas between 0.09 and $2.5 \mu \mathrm{m}^{2}$ and circularities between 0.5 and 1 , were counted and intensities measured. Scale bar $=10 \mu \mathrm{m}$. (C) The relative frequency distributions of normalized intensities show no significant difference in any region. 
The five intervals around the intersection of the frequency distributions or, alternatively, the common peak of WT and KO histograms were statistically analyzed. Bar graphs show the mean intensity of VGAT puncta, which were equally not significantly different in any region. (D) The mean puncta count per $100 \mu \mathrm{m}^{2}$ (\#) was not different in any stratum. (E) The mean area of puncta was not changed.

so, stratum oriens; sp, stratum pyramidale; sr, stratum radiatum; slm, stratum lacunosum moleculare. Error bars represent sem; all statistical analyses were carried out using the two-tailed Student's $t$-test; ns, -, not significant. 


\section{V.2.5.2. Biochemical Analysis of Postsynaptic Markers in the Rasgrp1 KO Hippocampus}

PSD-95 does not only act as a structural component at the PSD, but also regulates synaptic transmission by providing "slots" that allow AMPA receptors to be incorporated into the synapse. This "slot theory" was established after the observation that overexpression of PSD-95 selectively leads to increased AMPAR transmission (Béïque and Andrade, 2003; El-Husseini et al., 2000; Schnell et al., 2002). Conversely, reducing PSD-95 levels mediates a reduced AMPAR transmission (Elias et al., 2006; Elias and Nicoll, 2007; Schlüter et al., 2006). Recently, evidence was also provided for an indirect influence of PSD-95 on presynaptic release probability via its interaction with Neuroligins (Futai et al., 2007; Irie et al., 1997). Furthermore, increased levels of PSD-95 can influence NMDAR transmission (Futai et al., 2007).

First, PSD-95 expression was investigated. In hippocampal homogenates (Figure V.14 A), relative PSD-95 protein content was increased by $\sim 10 \%$ as compared to WT homogenate, but this difference was not statistically significant (homogenate; $\mathrm{WT}_{\mathrm{PSD}-95}=1.00 \pm 0.09 ; \mathrm{KO}_{\mathrm{PSD}-95}=1.10 \pm 0.08 ; n, \mathrm{WT}=15 ; n, \mathrm{KO}=$ $15 ; p=0.401)$. Contrastingly, the PSD-95 content was increased by $\sim 40 \%$ in the PSD fraction (Figure V.14 B; PSD fraction; $\mathrm{WT}_{\mathrm{PSD}-95}=1.00 \pm 0.09 ; \mathrm{KO}_{\mathrm{PSD}-95}=1.41 \pm$ $0.16 ; n, \mathrm{WT}=14 ; n, \mathrm{KO}=15 ;{ }^{* *} p=0.042$ )

In order to investigate, if this genotype specific increase of PSD-95 levels also involved abnormal levels of AMPARs and/or NMDARs, purified PSD fractions were analyzed for several AMPAR and NMDAR subunits. AMPA receptors are oligoheteromers composed of four subunits (Rosenmund et al., 1998). The preferred composition is that of two heterodimers, mostly GluR1/2 and in some cases, GluR2/3 (Derkach et al., 2007). GluR4 is not present in the adult hippocampus (Allen_Mouse_Brain_Atlas, 2009; Zhu et al., 2000) and therefore GluR4 expression was not assessed in this study. However, neither of the AMPAR subunits showed abnormal levels either in homogenates or in PSD fractions of Rasgrp1 KO hippocampus (Table V.12).

Next, NMDAR subunit expression was examined. NMDAR receptors are heterooligomers composed of four subunits. They contain two obligatory NMDAR1 subunits and two variable NMDAR2 or NMDAR3 subunits. The NMDAR2 family comprises of NMDAR2A, -B, $-C$ and $-D$, and the NMDAR3 family of NMDAR3A and B (Cull-Candy 
et al., 2001). Expression of NMDAR1, NMDAR2A and NMDAR2B were analyzed in hippocampal homogenates and PSD fractions of Rasgrp1 deficient mice, but no abnormal protein expression was found (Table V.12).

Table V.12: Relative protein content of markers in homogenate and PSD fractions

\begin{tabular}{|l|c|c|c|l|c|c|c|}
\hline \multicolumn{4}{|c|}{ homogenate (Figure V.14 A) } & \multicolumn{4}{c|}{ PSD fraction (Figure V.14 B) } \\
\hline marker & WT & KO & $p$ & marker & WT & KO & $p$ \\
\hline PSD-95 & $1.00 \pm 0.09$ & $1.10 \pm 0.08$ & 0.401 & PSD-95 & $1.00 \pm 0.09$ & $1.41 \pm 0.16$ & 0.042 \\
\hline GluR1 & $1.00 \pm 0.06$ & $1.03 \pm 0.04$ & 0.713 & GluR1 & $1.00 \pm 0.10$ & $1.12 \pm 0.16$ & 0.568 \\
\hline GluR2 & $1.00 \pm 0.06$ & $1.02 \pm 0.04$ & 0.653 & GluR2 & $1.00 \pm 0.16$ & $0.92 \pm 0.10$ & 0.330 \\
\hline GluR2/3 & $1.00 \pm 0.06$ & $0.98 \pm 0.06$ & 0.698 & GluR2/3 & $1.00 \pm 0.16$ & $1.01 \pm 0.14$ & 0.606 \\
\hline NMDAR1 & $1.00 \pm 0.05$ & $1.01 \pm 0.05$ & 0.767 & NMDAR1 & $1.00 \pm 0.18$ & $0.96 \pm 0.14$ & 0.376 \\
\hline NMDAR2A & $1.00 \pm 0.05$ & $0.95 \pm 0.05$ & 0.543 & NMDAR2A & $1.00 \pm 0.10$ & $1.05 \pm 0.10$ & 0.727 \\
\hline NMDAR2B & $1.00 \pm 0.06$ & $1.03 \pm 0.07$ & 0.538 & NMDAR2B & $1.00 \pm 0.19$ & $0.85 \pm 0.10$ & 0.640 \\
\hline
\end{tabular}

All values are ratios of densitometric readouts (marker/control) \pm sem. Statistical analyses were carried out using the two-tailed Students $t$-test; $n$ - numbers are found at the bottom of the corresponding bar graphs (Figure V.14).

In addition to the increased fluorescence signal of PSD-95 seen in immunohistochemically stained hippocampal sections (Figure V.10), these results confirm the hypothesis that PSD-95 expression is enhanced in Rasgrp1 $\mathrm{KO}$ animals. However, the analyses of homogenates and PSD fractions provide no indication for abnormal protein expression of the major glutamatergic receptor subunits. 
A

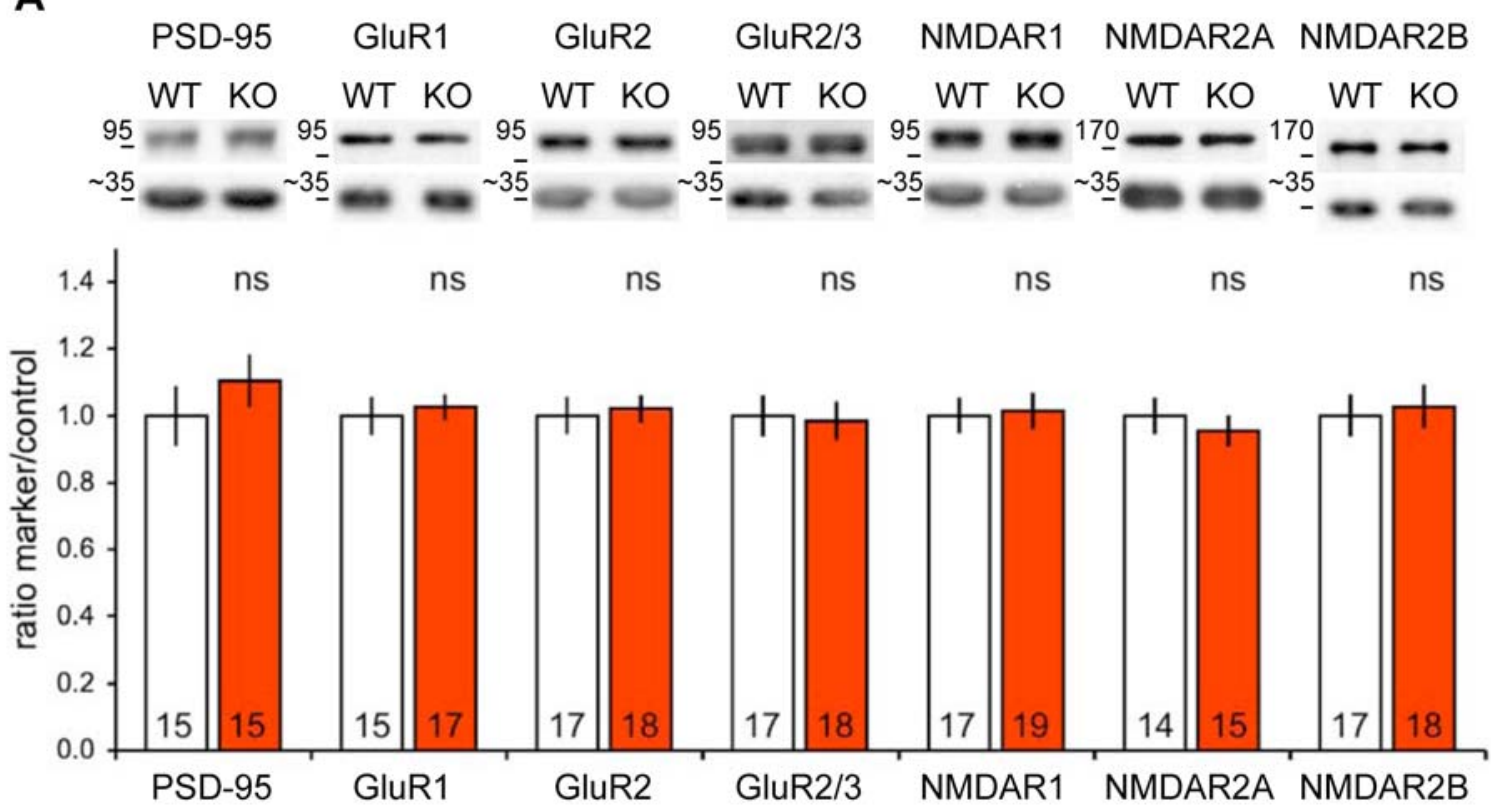

B

PSD-95 GluR1 GluR2 GluR2/3 NMDAR1 NMDAR2A NMDAR2B

WT KO WT KO WT KO WT KO WT KO WT KO WT KO

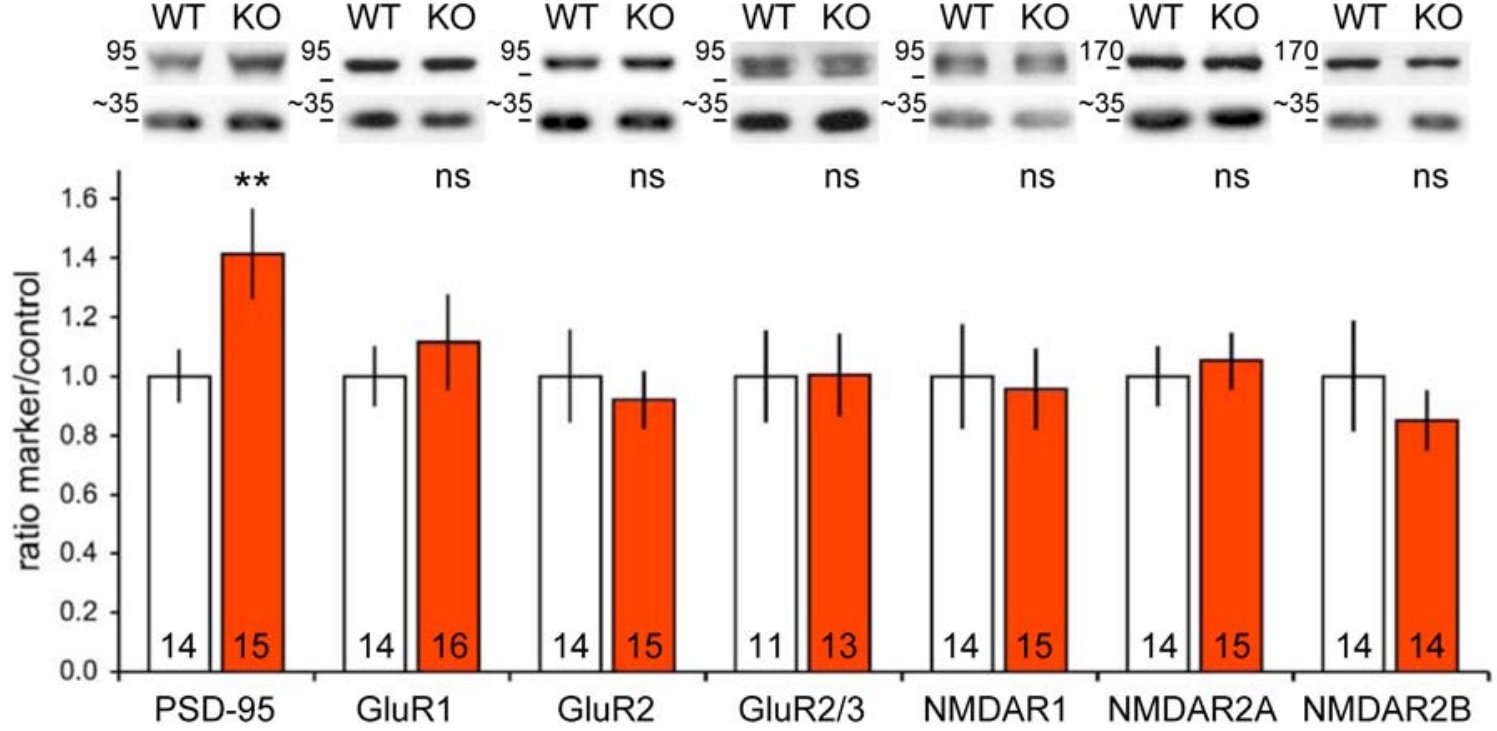

Figure V.14: Biochemical analysis of hippocampal homogenates and PSD fractions prepared from Rasgrp1 KO mice

The expression of PSD-95 and receptor subunits was assessed by densitometric analysis of immunolabeled Western blots. PSD-95 expression in postsynaptic density (PSD) fractions was significantly increased in Rasgrp1 knock-out (KO) hippocampi.

(A) Western blots of homogenates. Sample blots are shown on top of the corresponding bar graphs. Synaptophysin $(\sim 37 \mathrm{kDa})$ was used as control of total protein load. The ratio of densitometric values of marker and corresponding control 
are displayed in the bar graphs. None of the markers tested in Rasgrp1 KO homogenate showed a significant difference as compared to wild-type (WT).

(B) Western blots of PSD fractions. Sample blots and analyses of PSD-fraction are displayed as described in (A). PSD-95 expression was significantly increased in PSD fractions of Rasgrp1 KO hippocampi.

Numbers of animals (3-5 weeks old) analyzed are shown at the bottom of the bar graphs; error bars represent sem; statistical analyses were carried out using the twotailed Student's $t$-test $\left({ }^{* *}, p<0.01 ;\right.$ ns, not significant). 


\section{Discussion}

\section{VI.1. Lack of Rasgrp1 Increases Postsynaptic Sensitivity}

In the present study, I analyzed the effects of Rasgrp1 deficiency on hippocampal function. The results of this study indicate, that Rasgrp1 deficient mice exhibit an increased postsynaptic sensitivity.

The efficiency of synaptic transmission in the hippocampus of Rasgrp1 KO is increased by $35 \%$. This discovery was made in field recordings, applying an inputoutput protocol that correlated the input from Schaffer collaterals to resulting fEPSPS in the stratum radiatum of the CA1 region. An increase in synaptic transmission may result from three phenomena: An increase in synapses, increased number or release probability of synaptic vesicles, or increased sensitivity of postsynapses. PPF experiments as well as $14 \mathrm{~Hz}$ train experiment did not show any difference between WT and Rasgrp1 KO animals, indicating that presynaptic characteristics are not changed in Rasgrp1 KOs. In addition, normal mEPSC frequency indicated that the number of functional synapses is unchanged in Rasgrp1 KOs. In contrast, the amplitudes of mEPSC were increased in Rasgrp1 KO cells, indicating towards an increased postsynaptic sensitivity. A detailed immunohistochemical analysis showed that numbers and intensities of stained presynapses are unchanged or even slightly reduced in the hippocampus of Rasgrp1 KO animals. Excitatory presynapses (VGLUT1 stainings) showed normal numbers and intensities in the CA1 region, as was the case for inhibitory presynapses (VGAT stainings), indicating that the numbers of excitatory and inhibitory presynapses are not affected in Rasgrp1 KO mice. Also, comparative immunostainings showed that Rasgrp1 is not present in the somata of inhibitory neurons of the stratum pyramidale. Rather, Rasgrp1 appears to be exclusively present in somata of pyramidal neurons, and is therefore unlikely to function in the formation or regulation of inhibitory presynapses. The number of total presynapses (Synapsin1/2 stainings) was slightly reduced $(\sim 6 \%)$ in the CA1 region of Rasgrp1 KO animals. In any case, the present data show that the increase in synaptic transmission cannot be explained by an increase in the number of functional synapses.

In contrast, the numbers and intensities of postsynaptic puncta (PSD-95 stainings) were significantly increased in the CA1 region of Rasgrp1 KO mice. Together with the finding that numbers of presynapses are not increased in Rasgrp1 $\mathrm{KO}$ mice, these data likely result from an increased rate of detection of postsynaptic 
puncta due to higher intensity levels. In biochemical analyses of PSD fractions prepared from Rasgrp1 KO hippocampi, an increased expression of PSD-95 was found. These results lead to the conclusion that loss of Rasgrp1 leads to a strengthening of postsynaptic efficiency via an increase of PSD-95 levels at postsynapses. The slight but significant reductions of presynapses stained for Synapsin1/2 (see Figure V.11 D) may represent an indirect effect of postsynaptic strengthening. It has recently been shown, that high levels of PSD-95 can indirectly influence presynaptic function (Futai et al., 2007). Therefore, reduced protein levels of Synapsin1/2 might represent an adaptive effect to an increased synaptic transmission. Alternatively, the slight reduction in the number of presynaptic structures detected by Synapsin $1 / 2$ staining may not have been noticed in the VGLUT1 and VGAT staining experiments due to differences in the detection sensitivity. In addition, the Synapsin1/2 staining may have detected a small subset of non-glutamatergic/non-GABAergic synapses that cannot be detected by antibodies to VGLUT1 and VGAT.

\section{VI.2. An Increase of PSD-95 Levels in the Hippocampus of Rasgrp1 KO Mice Mediates Increased Synaptic Efficiency}

In addition to increased levels of PSD-95, Rasgrp1 KO neurons show an increased sensitivity towards L-AMPA. This was demonstrated by bath application of L-AMPA during patch-clamp recordings. It is generally believed, that PSD-95 scaffoldings at the PSD provide "slots" for the insertion of AMPA receptors and, the level of PSD-95 expression at the postsynapse is positively correlated to the level of synaptic transmission (Béïque and Andrade, 2003; El-Husseini et al., 2000; Elias et al., 2006; Elias and Nicoll, 2007; Schlüter et al., 2006; Schnell et al., 2002). However, no aberrant expression of AMPAR and NMDAR subunits was detected in purified PSD fractions of Rasgrp1 KO mice. There are at least four possible explanations for this finding, all of which may be relevant in the context of the present study.

First, the method that was used here to purify PSD fractions and the analysis by immunoblotting may not be sensitive enough to detect mild changes in protein levels. At their respective peaks, WT and KO L-AMPA- responses differ by $~ 25 \%$ (Figure V.9 D). Even if sensitivity towards L-AMPA were linearly proportional to the content of AMPA receptors at the synapse, this increase would by very difficult to detect by 
immunoblotting. Also, the nature of the "slots" provided for AMPA receptors by PSD scaffolds is not known yet. Estimated numbers for the content of scaffolding molecules at the postsynapse range from 60 to 400 , whereas the corresponding numbers of AMPA receptors only range from 5-200 (Newpher and Ehlers, 2008) indicating that the "slot" for one receptor is formed by a multitude of scaffolding molecules. This relation of scaffold molecules and receptors appears to be nonlinear. With regard to minimal values, this ratio might increase to 12 scaffold molecules per receptor, indicating that empty slots exist. Even in the case of an overabundance of AMPARs, these empty slots are not filled. This has been demonstrated by the finding, that overexpression of AMPAR subunits alone does not result in enhanced transmission (Shi et al., 2001), consistent with the idea that empty slots bind receptors only upon specific stimuli, e.g. during LTP (Lisman and Raghavachari, 2006). Although decreased, Rasgrp1 deficient mice still show LTP. It is likely, that increased basal synaptic transmission occludes the effect of potentiation. However, potentiation would not be maintained at all, if AMPARs were present at the postsynapse at the same number, as slots exist. These considerations might explain, why an increase in AMPAR levels at the postsynapse can be expected to be lower than a primary increase in PSD-95 levels and might therefore not detectable by biochemical analysis.

Second, an explanation for the discrepancy between PSD-95 changes and AMPAR levels may be provided by aberrant subunit composition of AMPARs in Rasgrp1 deficient mice. The presence or absence of a single AMPAR subunit can dramatically change synaptic transmission. For example, GluR1 is a target of Camk2 phosphorylation, which enhances the coupling efficiency and facilitates channel opening. Other studies showed that this effect is responsible for the significantly increased single-channel conductance of homomeric GluR1 AMPARs (Derkach et al., 1999; Derkach et al., 2007). In addition, AMPARs lacking GluR2 exhibit increased permeability for calcium, open probability and conductance (Derkach et al., 2007). Although statistically not significant, GluR1 expression in PSD fractions of Rasgrp1 KO hippocampi is increased by $\sim 12 \%$. Furthermore, GluR2 expression is reduced by $\sim 8 \%$. These tendencies might indicate a change in AMPAR subunit composition in Rasgrp1 deficient mice. Moreover, the response of Rasgrp1 KO neurons to wash-in of L-AMPA was not only increased in amplitude, but also displayed an earlier onset and an increased slope. Increased incorporation of GluR1 and decreased 
incorporation of GluR2 might indicate an enhanced efficiency of glutamate binding and channel opening and would explain earlier onset and increased slope of the LAMPA curve in Rasgrp1 KOs. However, the mild changes in subunit expression, which can lead to these dramatic changes in receptor properties, are unlikely to be detectable by immunoblotting.

Third, the increase in synaptic transmission and sensitivity towards L-AMPA seen in Rasgrp1 deficient mice may result from changed properties of an AMPAR population, that is "normal" at the level of expression. These changes may include posttranscriptional and posttranslational changes as well as phosphorylation. GluR1 phosphorylation and a resulting increase in conductance of GluR1 containing AMPARs have already been mentioned above as a possible mechanism (Derkach et al., 1999; Derkach et al., 2007). Many other enzymes are known to modulate AMPAR function by phosphorylation, including cAMP-dependent protein kinase (PKA) and protein kinase $C$ (PKC) (Song and Huganir, 2002). RNA processing events, including alternative splicing and RNA editing, are known to diversify AMPAR function. RNA splicing of mutually exclusive exons (termed flip/flop) within the Glutamate binding domain modulates desensitization kinetics (Greger et al., 2007). If and in which way these processes are influenced by a lack of Rasgrp1, remains to be clarified.

Fourth, presumably it is not possible to discriminate between synaptic and extrasynaptic AMPARs by analysis of PSD fractions, because NMDAR2B subunits were detected in this fraction (Figure V.14 B). The NMDAR2B and D subunits dominate the neonatal brain, but are replaced by NMDAR2A or NMDAR2C subunits during the course of development (Sheng et al., 1994). However, NMDAR1/NMDAR2B receptors are still present in mature neurons, but exclusively at extrasynaptic sites (Cull-Candy et al., 2001; Newpher and Ehlers, 2008). Therefore it is very likely that not only synaptic PSDs, but also extrasynaptic sites containing receptors are purified by the method used in the present study. These extrasynaptic sites are microdomains consisting of membranous and submembranous structures such as scaffoldings, and are thought to play a pivotal role in AMPAR dynamics. The loosely defined extrasynaptic region begins at the spine neck and encompasses dendritic and somatic plasma membrane (Newpher and Ehlers, 2008). AMPARs found in these extrasynaptic regions are assumed to function as a reserve pool and may be trafficked to the PSD by lateral diffusion within the plasma membrane or by endocytotic recycling. In Rasgrp1 KO hippocampi, synaptic AMPARs may be 
increased by the same amount as extrasynaptic AMPAR numbers are decreased. This transfer would be impossible to monitor by a method that cannot distinguish these receptor populations.

\section{VI.3. Ras Signaling and Synaptic Transmission}

Syngap1 is the only known Ras signaling protein that has been directly implicated in the function of PSD-95. However, it is unclear how this functional interplay operates mechanistically. Syngap1 is found at the postsynapse at similar amounts as PSD-95 and PSD-93 combined (Cheng et al., 2006) and interacts directly with PSD-95 (Feng and Zhang, 2009). In mature neurons, Syngap1 also associates with the NMDAR2B subunit of NMDARs (Derkach et al., 2007), and therefore should also be present at extrasynaptic sites. Given its abundance at the postsynapse, Syngap1 is also discussed to act as a scaffolding molecule. Camk2, another protein that is highly enriched at the postsynapse, regulates Syngap1 via phosphorylation, leading to an increase in GAP activity (Oh et al., 2004). Therefore an increase in calcium/Calmodulin by synaptic activity would reduce active Ras levels by means of Syngap1. Since Ras is thought to positively act on AMPAR trafficking, Syngap1 might limit AMPAR trafficking (Derkach et al., 2007). Given the high amount of protein found at the cytosolic face of the PSD, Syngap1, a negative regulator of Ras activity, can be imagined as a "barrier" for AMPAR trafficking mediated by active Ras. Considering only the level of Ras activity, an increase in synaptic transmission upon Syngap1 deficiency is contradictory to the findings of the present study, since a decreased level of Ras activity is expected in cells deficient of Rasgrp1, yet AMPAR mediated transmission is increased.

Gain-of-function studies involving Hras (i.e. overexpression of G12V Hras) and loss-of-function studies involving Syngap1 yielded more or less congruent results. Overexpression of G12V Hras results in enhanced synaptic transmission (Imamura et al., 2003; Rumbaugh et al., 2006; Zhu et al., 2002). These findings shaped the general view on Ras as a positive regulator of AMPAR trafficking. Other findings are not as congruent with this general view of Ras signaling in neurons. An AMPAR mediated enhancement in transmission was observed in mice deficient in Rasgrf1 (Brambilla et al., 1997; Tonini et al., 2001) and, Hras KO mice exhibit normal AMPAR mediated transmission (Manabe et al., 2000). The electrophysiological phenotype of the Rasgrf1 KO mouse, which was characterized by Brambilla et al. (1997), is similar 
to that of the Rasgrp1 KO mouse described here. The present study and the study of Brambilla et al. (1997) indicate that not only increased but also decreased Ras activity can enhance synaptic transmission.

Looking more closely at the general interpretation according to which Ras activity positively regulates synaptic transmission, it appears, that this view currently lacks an explanation that involves definitive molecular and cellular mechanisms. In research on Ras signaling effects at the postsynapse, some researchers stress the ability of Ras $G$ proteins to positively act in the generation of new dendritic spines (Seeger et al., 2005; Vazquez et al., 2004) while others highlight the role in AMPAR trafficking and exclude the generation of new spines (Imamura et al., 2003; Rumbaugh et al., 2006; Zhu et al., 2002). However, the data backing these opposing views do not provide an insight into the mechanisms by which Ras signaling is acting at the molecular or cellular level. In contrast, known interactions, effector cascades, and regulatory proteins can clearly associate Rho $\mathrm{G}$ proteins with a regulation of the actin cytoskeleton, also during spine morphogenesis (Yoshihara et al., 2009). Mechanisms of Rab $G$ proteins in endocytosis and vesicle trafficking have been studied in detail, revealing their role in AMPAR endocytosis ( $\mathrm{Ng}$ and Tang, 2008). Research on Ras signaling in neurons lacks this background in many respects. In particular, none of the studies investigating Ras signaling in AMPAR trafficking take into account a possible role of Ras in the trafficking of PSD scaffolds.

As already described above (see chapter II.5), a strong determinant of Ras function seems to be the subcellular location, at which Ras is activated.

\section{VI.4. Localized Regulation of Neuronal Ras Function}

Syngap1 is not present in the somata of adult pyramidal neurons (Moon et al., 2008), and apart from the PSD, Syngap1 is only found in the dendritic shaft (Barnett et al., 2006). Given the almost exclusive localization of Syngap1 to postsynapses, inactivation of Ras activity by Syngap1 must be seen as a PSD specific form of Ras signaling. Overexpression of G12V Hras, as has been the usual experimental practice in studies on Ras signaling in AMPAR trafficking, "floods" the cell with active Ras. It is obvious, that this leads to a general perturbation of localization specific effects and to ectopic Ras signaling.

Rasgrp1 is almost exclusively localized to the Golgi apparatus. As in other cell types, the Golgi apparatus in neurons serves posttranslational modification of 
proteins and is the starting point for trafficking of proteins and membranes to other subcellular compartments and the plasma membrane. Because neurons are highly complex cells, the organization of their organelles differs from that of other cells in many respects. Dendrites are endowed with a satellite endomembranous system, comprising of Golgi outposts and SER, which is continuously linked to somatic ER (Kennedy and Ehlers, 2006; Tang, 2008). These satellite organelles are thought to act in local protein synthesis, modification and trafficking. Most Golgi outposts are found in the apical dendrite of pyramidal neurons, up to $100 \mu \mathrm{m}$ away from the soma and seem to localize primarily at branch points (Horton et al., 2005). In the present study, Rasgrp1 immunofluorescence was also found in proximal dendrites and seemed to be enriched in branch points (Figure V.1). However, the maximal distance from the soma at which these signals were found was $\sim 40 \mu \mathrm{m}$. Furthermore, these accumulations of Rasgrp1 exhibited very low intensities in comparison to the intensity of immunofluorescence in the soma. In contrast, effects of Rasgrp1 deficiency, i.e. increased numbers and intensities of PSD-95 puncta in the stratum lacunosum moleculare are found up to $200 \mu \mathrm{m}$ away from the soma (Figure V.10). It seems unlikely, that Rasgrp1 regulates these distant effects directly from its localization in the soma. Unlike Syngap1, which is almost exclusively found at the postsynapse, Rasgrp1 may rather regulate the development of postsynapses and the supply of PSD-95 in adult stages. Given this segregation of Rasgrp1 and Syngap1 function, completely opposite regulation (i.e. activation/inactivation) of the same downstream effector leading to the same physiological effect (i.e. negative regulation of synaptic transmission) is plausible.

\section{VI.5. A Possible Role for Rasgrp1 in Synaptogenesis}

Initial synaptogenesis is a process that occurs within the period in which both Rasgrp1 and PSD-95 levels were shown to increase. Proteins involved in synaptogenesis are present in neurons before synapses are formed. They are localized to heterogeneous clusters of proteins, called "packets", in dendrites and axons (McAllister, 2007).

Upon axo-dendritic contact, the first proteins to arrive at the future postsynapse are NMDARs. Arrival of PSD-95 occurs with a high degree of temporal variability, i.e. as early as NMDARs are detected at synapses or up to one hour later. However, AMPARs are incorporated into the new structure only after PSD-95 has accumulated 
at the postsynapse (Bresler et al., 2001; Friedman et al., 2000; McAllister, 2007). This is in accord with the notion that scaffoldings have to be present for synapse incorporation of AMPARs (Newpher and Ehlers, 2008). NMDARs and PSD-95 are transported in discrete packages, and AMPARs are transported in a subset of NMDAR containing packages (McAllister, 2007). This form of trafficking continues during development as well as in maturity (Gerrow et al., 2006). Observation of these transport packages using time-lapse live imaging has so far focused on their movement in dendrites, without investigating how and where they are formed exactly. However, an earlier study on developing cultured neurons showed, that PSD-95 locates to Golgi and endomembranous structures at DIV1; additional clustering in dendrites was observed from DIV4 onwards (El-Husseini et al., 2000). The specific localization of Rasgrp1 to the Golgi apparatus and presumably to proximal Golgi outposts is compatible with the idea that Rasgrp1 might in some way regulate the formation of PSD-95 containing transport packages. The different packaging and transport of AMPARs and PSD-95 might also represent different forms of Ras signaling. Syngap1/Ras signaling might be involved in AMPAR trafficking, while Rasgrp1/Ras signaling might be more important for trafficking of PSD-95.

Recently, a particular finding was published (Kim et al., 2009), that might link Rasgrp1 to PSD-95 and the regulation of synaptic transmission. Rasgrp1 is activated by diacylglycerol (DAG) (Dower et al., 2000; Ebinu et al., 1998; Ebinu et al., 2000; Kawasaki et al., 1998; Lorenzo et al., 2001; Reuther et al., 2002). DAG kinases (Dgks) are enzymes that remove DAG by converting it to phosphaditic acid (PA). Kim et al. (2009) showed, that KO of DAG kinase zeta (Dgkz) leads to decreased synaptic transmission and reduced spine density. The authors describe a mechanism by which PSD-95 recruits Dgkz to the postsynapse to tightly control local DAG levels. However, the initial localization of Dgkz is in the soma and in the proximal dendrites, as shown in images of the study by Kim et al. (2009). This subcellular localization resembles that of Rasgrp1. As Dgkz has the potential to reduce Rasgrp1 activity, this regulation might allow PSD-95 to exit the soma and proximal dendrites, leading to the formation of postsynapses in more distal regions. In case of a DGKz deficiency, this regulation is inhibited and PSD-95 is maintained in the soma and does not form or enhance postsynapses. Conversely, by KO of Rasgrp1, negative regulation is lacking and increased amounts of PSD-95 are trafficked into the dendrite, leading to the opposite phenotype. 


\section{VI.6. A Possible Role of Rasgrp1 in Dendrite Morphogenesis}

I also showed in the present study that Rasgrp1 deficient neurons display defects in dendrite morphogenesis. At DIV5, the complexity of Rasgrp1 KO hippocampal neurons was slightly, but significantly decreased. However, no aberrant morphology was observed at DIV10. At DIV 14 and 21, even an increase in dendritic complexity was detected.

When cultured, neurons develop in well-defined stages and in a stereotypic fashion (Bradke and Dotti, 2000; Dotti et al., 1988). Shortly after plating, the cells attach and form a lamellopodium around the cell. At DIV1, this lamellopodium condenses and forms several neurites. Within $24 \mathrm{~h}$, one of these neurites starts to grow very rapidly, whereas the others stay quiescent. This process is called "symmetry braking" and defines neuronal polarity. The growing neurite becomes the axon at later stages. Growth of neurites, which later shape the dendritic tree, starts at DIV4.

In the hippocampus, Rasgrp1 is detectable at late embryonal stages in the subiculum, but is not detectable in the hippocampus until P2 (Pierret et al., 2001). Also in neurons cultured from mouse hippocampi, staining for Rasgrp1 detected the protein as early as DIV3 (data not shown). In this respect, it is likely that rather than in early polarization, Rasgrp1 functions in dendrite growth. Dendrite growth is most rapid between DIV4 and DIV10. After DIV10, the development of dendritic complexity slows down (Dotti et al., 1988). Interestingly, dendritic complexity is reduced at later stages until it stabilizes at slightly lower levels (Wong and Ghosh, 2002). This process is called "dendritic pruning" and is thought to occur upon synapse formation. Only the branches that are contacted by the appropriate inputs are maintained and stabilized while others are lost.

Sholl analyses revealed, that WT neurons at DIV14 are slightly more complex at distances larger than $75 \mu \mathrm{m}$ from the soma than WT neurons at DIV21 (Figure V.7 C, D). This result may reflect dendrite pruning between DIV14 and DIV21. In view of this finding, enhanced dendrite complexity of Rasgrp1 KO neurons at both stages should not only be discussed as a defect in dendrite growth but also as a putative defect in dendrite pruning. Additionally, it remains to be investigated if Rasgrp1 KO mice display a defect in morphology in-vivo. It has not been determined yet if the increased numbers of PSD-95 positive puncta in the hippocampus of Rasgrp1 KOs reflect an increase in postsynapses (see chapter VI.1). If so, this might result from an 
enlargement of dendrites. However, it is important to note that a putative effect on dendrite morphology does not have an effect on synaptic integrity of the hippocampus, as no change in the number of functional synapses was detected by imaging (Figure V.10 - V.13) or by patch clamp experiments (Figure V.9). Also, the increased L-AMPA sensitivity of cells deficient of Rasgrp1 strongly supports the notion, that defects in the regulation of receptors might be the primary cause for enhanced synaptic transmission seen in Rasgrp1 KO neurons, and not aberrant dendrite morphology.

In contrast to the analysis of dendrite morphogenesis in-vitro, the functional characterization of Rasgrp1 KO mice represents only one time point of observation. The latter results represent the endpoint of a period of aberrant development, i.e. from P2 onwards (the postnatal day at which Rasgrp1 can normally be detected in the hippocampus) to the time point of observation. The analysis is therefore ignorant of the changes that underlie early development until the time-period of observation, i.e. the late juvenile stage. Although more slowly and with less dramatic changes, development still proceeds after this stage. PSD-95 is expressed from embryonal stages onwards in the developing hippocampus, although the level of expression does not increase steadily (Fukaya et al., 1999). From P2 to P7, the expression level of PSD-95 decreases in all layers of the CA1 region. After P7, PSD-95 levels increase in the stratum radiatum and stratum lacunosum moleculare until P28. After P28, protein levels decrease by $\sim 25 \%$ until P56 (Chang et al., 2009).

The maturation of the brain also involves regressive events, such as the loss of synapses and stabilization of the remaining ones. This is accompanied and determined by the reduction of expression of specific proteins, e.g. PSD-95. As mentioned above, also dendrite development is characterized by periods of regressive events such as dendrite pruning (Wong and Ghosh, 2002).

In dendrite morphogenesis, young hippocampal neurons obtained from Rasgrp1 KO animals first show a decreased dendrite complexity. However at adult stages, their complexity is increased as compared to control neurons. It is possible that neurons deficient in Rasgrp1 somehow develop more slowly than WT neurons, with respect to both growth and regressive events, and that dendrite growth is first delayed while dendrite pruning is impaired subsequently. Might the decrease of PSD95 expression after P28, as seen in immunolabelings observed by Chang et al. (2009) also be delayed in Rasgrp1 KO animals? Rasgrp1 KO animals were analyzed 
at a time when PSD-95 levels in WT animals start to decrease. Thus the enhanced intensity of PSD-95 immunostainings in Rasgrp1 KOs would also represent "delayed" synaptic weakening rather than abnormal synaptic enhancement. To test this directly, the developmental time course of PSD-95 expression in Rasgrp1 KO hippocampi would have to be analyzed.

\section{VI.7. Outlook}

Based on the present findings, two major future research directions should be pursued with regard to Ras signaling in neurons and the specific role of Rasgrp1 in this process.

First, the tissue-, cell- and compartment- specific localizations of Ras G proteins and their regulatory proteins have to be analyzed in much more detail. Existing data are either scarce or were obtained with methods that do not allow comparative analyses of Ras signaling factors and marker proteins (e.g. immunostainings for light microscopy). Nowadays, a large number of specific antibodies for such marker proteins are available. Furthermore, research at this general level should focus on the molecular machineries that the effects of Ras signaling rely on in neurons. The canonical Raf/Map2k/Mapk, Pi3k and Ralgds pathways and alterations of gene expression cannot alone explain the pleiotropic effects that Ras signaling appears to have in neurons.

Second, research on Rasgrp1 should focus on the molecular networks and cellular processes that control expression or trafficking of scaffold molecules. This should reveal the mechanisms that cause the phenotypic changes seen in Rasgrp1 deficient mice. Corresponding approaches should examine the neuronal factors that regulate Rasgrp1, the compartments that Rasgrp1 translocates to upon activation, and the proteins that interact with Rasgrp1.

In combination, these two research directions will not only lead to a more detailed understanding of Rasgrp1 alone, but also to a better understanding of Ras signaling in neurons in general. 


\section{Literature}

Abrams, C.S. (2005). Intracellular signaling in platelets. Curr Opin Hematol 12, 401405.

Ahn, N.G. (1993). The MAP kinase cascade. Discovery of a new signal transduction pathway. Mol Cell Biochem 127-128, 201-209.

Allen_Mouse_Brain_Atlas (2009). Allen Mouse Brain Atlas [Internet]. Seattle (WA): Allen Institute for Brain Science. (C2009. Available from: http://mouse.brain-map.org.

Alpar, A., Palm, K., Schierwagen, A., Arendt, T., and Gartner, U. (2003). Expression of constitutively active p21H-rasval12 in postmitotic pyramidal neurons results in increased dendritic size and complexity. J Comp Neurol 467, 119-133.

Amaral, D.G., and Witter, M.P. (1989). The three-dimensional organization of the hippocampal formation: a review of anatomical data. Neuroscience 31, 571-591.

Arai, J.A., Li, S., and Feig, L.A. (2009). Sos2 is dispensable for NMDA-induced Erk activation and LTP induction. Neurosci Lett 455, 22-25.

Arendt, T., Gartner, U., Seeger, G., Barmashenko, G., Palm, K., Mittmann, T., Yan, L., Hummeke, M., Behrbohm, J., Bruckner, M.K., et al. (2004). Neuronal activation of Ras regulates synaptic connectivity. Eur J Neurosci 19, 2953-2966.

Augsten, M., Pusch, R., Biskup, C., Rennert, K., Wittig, U., Beyer, K., Blume, A., Wetzker, R., Friedrich, K., and Rubio, I. (2006). Live-cell imaging of endogenous Ras-GTP illustrates predominant Ras activation at the plasma membrane. EMBO Rep 7, 46-51.

Baba, H., Fuss, B., Urano, J., Poullet, P., Watson, J.B., Tamanoi, F., and Macklin, W.B. (1995). Gaplll, a new brain-enriched member of the GTPase-activating protein family. J Neurosci Res 41, 846-858.

Bading, H., Ginty, D.D., and Greenberg, M.E. (1993). Regulation of gene expression in hippocampal neurons by distinct calcium signaling pathways. Science 260, 181186.

Bading, H., and Greenberg, M.E. (1991). Stimulation of protein tyrosine phosphorylation by NMDA receptor activation. Science 253, 912-914.

Bar-Sagi, D., and Feramisco, J.R. (1985). Microinjection of the ras oncogene protein into PC12 cells induces morphological differentiation. Cell 42, 841-848.

Barnett, M.W., Watson, R.F., Vitalis, T., Porter, K., Komiyama, N.H., Stoney, P.N., Gillingwater, T.H., Grant, S.G., and Kind, P.C. (2006). Synaptic Ras GTPase 
activating protein regulates pattern formation in the trigeminal system of mice. $\mathrm{J}$ Neurosci 26, 1355-1365.

Beaulieu, N., Zahedi, B., Goulding, R.E., Tazmini, G., Anthony, K.V., Omeis, S.L., de Jong, D.R., and Kay, R.J. (2007). Regulation of RasGRP1 by B cell antigen receptor requires cooperativity between three domains controlling translocation to the plasma membrane. Mol Biol Cell 18, 3156-3168.

Béïque, J.C., and Andrade, R. (2003). PSD-95 regulates synaptic transmission and plasticity in rat cerebral cortex. The Journal of Physiology 546, 859-867.

Bellocchio, E.E., Hu, H., Pohorille, A., Chan, J., Pickel, V.M., and Edwards, R.H. (1998). The localization of the brain-specific inorganic phosphate transporter suggests a specific presynaptic role in glutamatergic transmission. J Neurosci 18, 8648-8659.

Bergmeier, W., Goerge, T., Wang, H.W., Crittenden, J.R., Baldwin, A.C., Cifuni, S.M., Housman, D.E., Graybiel, A.M., and Wagner, D.D. (2007). Mice lacking the signaling molecule CaIDAG-GEFI represent a model for leukocyte adhesion deficiency type III. J Clin Invest 117, 1699-1707.

Bernards, A. (2006). Ras superfamily and interacting proteins database. Meth Enzymol 407, 1-9.

Bernhardt, R., and Matus, A. (1984). Light and electron microscopic studies of the distribution of microtubule-associated protein 2 in rat brain: a difference between dendritic and axonal cytoskeletons. J Comp Neurol 226, 203-221.

Bilasy, S.E., Satoh, T., Ueda, S., Wei, P., Kanemura, H., Aiba, A., Terashima, T., and Kataoka, T. (2009). Dorsal telencephalon-specific RA-GEF-1 knockout mice develop heterotopic cortical mass and commissural fiber defect. Eur J Neurosci 29, 19942008.

Bivona, T.G., Perez De Castro, I., Ahearn, I.M., Grana, T.M., Chiu, V.K., Lockyer, P.J., Cullen, P.J., Pellicer, A., Cox, A.D., and Philips, M.R. (2003). Phospholipase Cgamma activates Ras on the Golgi apparatus by means of RasGRP1. Nature 424, 694-698.

Borasio, G.D., John, J., Wittinghofer, A., Barde, Y.A., Sendtner, M., and Heumann, R. (1989). ras p21 protein promotes survival and fiber outgrowth of cultured embryonic neurons. Neuron 2, 1087-1096.

Boriack-Sjodin, P.A., Margarit, S.M., Bar-Sagi, D., and Kuriyan, J. (1998). The structural basis of the activation of Ras by Sos. Nature 394, 337-343.

Bos, J.L., Rehmann, H., and Wittinghofer, A. (2007). GEFs and GAPs: critical elements in the control of small G proteins. Cell 129, 865-877. 
Bradke, F., and Dotti, C.G. (2000). Establishment of neuronal polarity: lessons from cultured hippocampal neurons. Curr Opin Neurobiol 10, 574-581.

Brambilla, R., Gnesutta, N., Minichiello, L., White, G., Roylance, A.J., Herron, C.E., Ramsey, M., Wolfer, D.P., Cestari, V., Rossi-Arnaud, C., et al. (1997). A role for the Ras signalling pathway in synaptic transmission and long-term memory. Nature 390, 281-286.

Bresler, T., Ramati, Y., Zamorano, P.L., Zhai, R., Garner, C.C., and Ziv, N.E. (2001). The dynamics of SAP90/PSD-95 recruitment to new synaptic junctions. Mol Cell Neurosci 18, 149-167.

Brown, M.D., and Sacks, D.B. (2009). Protein scaffolds in MAP kinase signalling. Cell Signal 21, 462-469.

Buday, L., and Downward, J. (2008). Many faces of Ras activation. Biochim Biophys Acta 1786, 178-187.

Burwell, R.D., Witter, M.P., and Amaral, D.G. (1995). Perirhinal and postrhinal cortices of the rat: a review of the neuroanatomical literature and comparison with findings from the monkey brain. Hippocampus 5, 390-408.

Carlisle, H.J., Manzerra, P., Marcora, E., and Kennedy, M.B. (2008). SynGAP regulates steady-state and activity-dependent phosphorylation of cofilin. J Neurosci 28, 13673-13683.

Cen, H., Papageorge, A.G., Zippel, R., Lowy, D.R., and Zhang, K. (1992). Isolation of multiple mouse cDNAs with coding homology to Saccharomyces cerevisiae CDC25: identification of a region related to Bcr, Vav, Dbl and CDC24. EMBO J 11, 40074015.

Cesca, F., Baldelli, P., Valtorta, F., and Benfenati, F. (2010). The synapsins: Key actors of synapse function and plasticity. Progress in neurobiology.

Chakraborty, A.K., Das, J., Zikherman, J., Yang, M., Govern, C.C., Ho, M., Weiss, A., and Roose, J. (2009). Molecular origin and functional consequences of digital signaling and hysteresis during Ras activation in lymphocytes. Science signaling 2, pt2.

Chang, L.R., Liu, J.P., Zhang, N., Wang, Y.J., Gao, X.L., and Wu, Y. (2009). Different expression of NR2B and PSD-95 in rat hippocampal subregions during postnatal development. Microsc Res Tech 72, 517-524.

Chaudhry, F.A., Reimer, R.J., Bellocchio, E.E., Danbolt, N.C., Osen, K.K., Edwards, R.H., and Storm-Mathisen, J. (1998). The vesicular GABA transporter, VGAT, localizes to synaptic vesicles in sets of glycinergic as well as GABAergic neurons. $J$ Neurosci 18, 9733-9750. 
Chen, H., Karam, J.A., Schultz, R., Zhang, Z., Duncan, C., and Hsieh, J.T. (2006). Cloning of mouse Dab2ip gene, a novel member of the RasGTPase-activating protein family and characterization of its regulatory region in prostate. DNA Cell Biol 25, 232-245.

Chen, H.J., Rojas-Soto, M., Oguni, A., and Kennedy, M.B. (1998). A synaptic RasGTPase activating protein (p135 SynGAP) inhibited by CaM kinase II. Neuron 20, 895-904.

Cheng, D., Hoogenraad, C.C., Rush, J., Ramm, E., Schlager, M.A., Duong, D.M., Xu, P., Wijayawardana, S.R., Hanfelt, J., Nakagawa, T., et al. (2006). Relative and absolute quantification of postsynaptic density proteome isolated from rat forebrain and cerebellum. Mol Cell Proteomics 5, 1158-1170.

Cho, K.O., Hunt, C.A., and Kennedy, M.B. (1992). The rat brain postsynaptic density fraction contains a homolog of the Drosophila discs-large tumor suppressor protein. Neuron 9, 929-942.

Choy, E., Chiu, V.K., Silletti, J., Feoktistov, M., Morimoto, T., Michaelson, D., Ivanov, I.E., and Philips, M.R. (1999). Endomembrane trafficking of ras: the CAAX motif targets proteins to the ER and Golgi. Cell 98, 69-80.

Clyde-Smith, J., Silins, G., Gartside, M., Grimmond, S., Etheridge, M., Apolloni, A., Hayward, N., and Hancock, J.F. (2000). Characterization of RasGRP2, a plasma membrane-targeted, dual specificity Ras/Rap exchange factor. J Biol Chem 275, 32260-32267.

Costa, R.M., Federov, N.B., Kogan, J.H., Murphy, G.G., Stern, J., Ohno, M., Kucherlapati, R., Jacks, T., and Silva, A.J. (2002). Mechanism for the learning deficits in a mouse model of neurofibromatosis type 1 . Nature $415,526-530$.

Craven, S.E., El-Husseini, A.E., and Bredt, D.S. (1999). Synaptic targeting of the postsynaptic density protein PSD-95 mediated by lipid and protein motifs. Neuron 22, 497-509.

Crittenden, J.R., Cantuti-Castelvetri, I., Saka, E., Keller-McGandy, C.E., Hernandez, L.F., Kett, L.R., Young, A.B., Standaert, D.G., and Graybiel, A.M. (2009). Dysregulation of CaIDAG-GEFI and CaIDAG-GEFII predicts the severity of motor side-effects induced by anti-parkinsonian therapy. Proc Natl Acad Sci USA.

Cui, Y., Costa, R.M., Murphy, G.G., Elgersma, Y., Zhu, Y., Gutmann, D.H., Parada, L.F., Mody, I., and Silva, A.J. (2008). Neurofibromin regulation of ERK signaling modulates GABA release and learning. Cell 135, 549-560.

Cull-Candy, S., Brickley, S., and Farrant, M. (2001). NMDA receptor subunits: diversity, development and disease. Current Opinion in Neurobiology 11, 327-335. 
Cullen, P.J., Hsuan, J.J., Truong, O., Letcher, A.J., Jackson, T.R., Dawson, A.P., and Irvine, R.F. (1995). Identification of a specific Ins(1,3,4,5)P4-binding protein as a member of the GAP1 family. Nature 376, 527-530.

DeFeo, D., Gonda, M.A., Young, H.A., Chang, E.H., Lowy, D.R., Scolnick, E.M., and Ellis, R.W. (1981). Analysis of two divergent rat genomic clones homologous to the transforming gene of Harvey murine sarcoma virus. Proc Natl Acad Sci USA 78, 3328-3332.

Derkach, V., Barria, A., and Soderling, T.R. (1999). Ca2+/calmodulin-kinase II enhances channel conductance of alpha-amino-3-hydroxy-5-methyl-4isoxazolepropionate type glutamate receptors. Proc Natl Acad Sci USA 96, 32693274.

Derkach, V.A., Oh, M.C., Guire, E.S., and Soderling, T.R. (2007). Regulatory mechanisms of AMPA receptors in synaptic plasticity. Nat Rev Neurosci 8, 101-113.

Dotti, C.G., Sullivan, C.A., and Banker, G. (1988). The establishment of polarity by hippocampal neurons in culture. J Neurosci 8, 1454-1468.

Dower, N.A., Stang, S.L., Bottorff, D.A., Ebinu, J.O., Dickie, P., Ostergaard, H.L., and Stone, J.C. (2000). RasGRP is essential for mouse thymocyte differentiation and TCR signaling. Nat Immunol 1, 317-321.

Ebinu, J.O., Bottorff, D.A., Chan, E.Y., Stang, S.L., Dunn, R.J., and Stone, J.C. (1998). RasGRP, a Ras guanyl nucleotide- releasing protein with calcium- and diacylglycerol-binding motifs. Science 280, 1082-1086.

Ebinu, J.O., Stang, S.L., Teixeira, C., Bottorff, D.A., Hooton, J., Blumberg, P.M., Barry, M., Bleakley, R.C., Ostergaard, H.L., and Stone, J.C. (2000). RasGRP links Tcell receptor signaling to Ras. Blood 95, 3199-3203.

Ehrhardt, A., Ehrhardt, G.R., Guo, X., and Schrader, J.W. (2002). Ras and relatives-job sharing and networking keep an old family together. Exp Hematol 30, 1089-1106.

El-Husseini, A.E., Schnell, E., Chetkovich, D.M., Nicoll, R.A., and Bredt, D.S. (2000). PSD-95 involvement in maturation of excitatory synapses. Science 290, 1364-1368.

Elias, G.M., Funke, L., Stein, V., Grant, S.G., Bredt, D.S., and Nicoll, R.A. (2006). Synapse-specific and developmentally regulated targeting of AMPA receptors by a family of MAGUK scaffolding proteins. Neuron 52, 307-320.

Elias, G.M., and Nicoll, R.A. (2007). Synaptic trafficking of glutamate receptors by MAGUK scaffolding proteins. Trends Cell Biol 17, 343-352.

Endo, M., and Yamashita, T. (2009). Inactivation of Ras by p120GAP via focal adhesion kinase dephosphorylation mediates RGMa-induced growth cone collapse. J Neurosci 29, 6649-6662. 
English, J.D., and Sweatt, J.D. (1996). Activation of p42 mitogen-activated protein kinase in hippocampal long term potentiation. J Biol Chem 271, 24329-24332.

Erlander, M.G., Tillakaratne, N.J., Feldblum, S., Patel, N., and Tobin, A.J. (1991). Two genes encode distinct glutamate decarboxylases. Neuron 7, 91-100.

Esclapez, M., Tillakaratne, N.J., Kaufman, D.L., Tobin, A.J., and Houser, C.R. (1994). Comparative localization of two forms of glutamic acid decarboxylase and their mRNAs in rat brain supports the concept of functional differences between the forms. J Neurosci 14, 1834-1855.

Esteban, L.M., Fernández-Medarde, A., López, E., Yienger, K., Guerrero, C., Ward, J.M., Tessarollo, L., and Santos, E. (2000). Ras-guanine nucleotide exchange factor sos2 is dispensable for mouse growth and development. Molecular and Cellular Biology 20, 6410-6413.

Evergren, E., Benfenati, F., and Shupliakov, O. (2007). The synapsin cycle: a view from the synaptic endocytic zone. J Neurosci Res 85, 2648-2656.

Feng, W., and Zhang, M. (2009). Organization and dynamics of PDZ-domain-related supramodules in the postsynaptic density. Nat Rev Neurosci 10, 87-99.

Fernández-Medarde, A., Esteban, L.M., Núñez, A., Porteros, A., Tessarollo, L., and Santos, E. (2002). Targeted disruption of Ras-Grf2 shows its dispensability for mouse growth and development. Molecular and Cellular Biology 22, 2498-2504.

Ferreira, A., Kao, H.T., Feng, J., Rapoport, M., and Greengard, P. (2000). Synapsin III: developmental expression, subcellular localization, and role in axon formation. J Neurosci 20, 3736-3744.

Finkbeiner, S., and Greenberg, M.E. (1996). Ca(2+)-dependent routes to Ras: mechanisms for neuronal survival, differentiation, and plasticity? Neuron 16, 233236.

Fivaz, M., Bandara, S., Inoue, T., and Meyer, T. (2008). Robust neuronal symmetry breaking by Ras-triggered local positive feedback. Curr Biol 18, 44-50.

Friedman, H.V., Bresler, T., Garner, C.C., and Ziv, N.E. (2000). Assembly of new individual excitatory synapses: time course and temporal order of synaptic molecule recruitment. Neuron 27, 57-69.

Fukaya, M., Ueda, H., Yamauchi, K., Inoue, Y., and Watanabe, M. (1999). Distinct spatiotemporal expression of mRNAs for the PSD-95/SAP90 protein family in the mouse brain. Neuroscience Research 33, 111-118.

Futai, K., Kim, M.J., Hashikawa, T., Scheiffele, P., Sheng, M., and Hayashi, Y. (2007). Retrograde modulation of presynaptic release probability through signaling mediated by PSD-95-neuroligin. Nat Neurosci 10, 186-195. 
Gärtner, U., Alpár, A., Behrbohm, J., Heumann, R., and Arendt, T. (2005). Enhanced Ras activity promotes spine formation in synRas mice neocortex. Neuroreport 16, 149-152.

Gerrow, K., Romorini, S., Nabi, S.M., Colicos, M.A., Sala, C., and El-Husseini, A. (2006). A preformed complex of postsynaptic proteins is involved in excitatory synapse development. Neuron 49, 547-562.

Goldfinger, L.E. (2008). Choose your own path: specificity in Ras GTPase signaling. Mol Biosyst 4, 293-299.

Golgi, C. (1873). Sulla struttura della grigia del cervello. Gazetta Medica Italiana, reviewd in 'Minds behind the Brain - A history of the Pioneers and Their Discoveries' by Stanley Finger, PhD, Oxford University Press

0, 204-206.

Gotoh, T., Niino, Y., Tokuda, M., Hatase, O., Nakamura, S., Matsuda, M., and Hattori, S. (1997). Activation of R-Ras by Ras-guanine nucleotide-releasing factor. J Biol Chem 272, $18602-18607$.

Greger, I.H., Ziff, E.B., and Penn, A.C. (2007). Molecular determinants of AMPA receptor subunit assembly. Trends Neurosci 30, 407-416.

Guerrero, I., Wong, H., Pellicer, A., and Burstein, D.E. (1986). Activated N-ras gene induces neuronal differentiation of PC12 rat pheochromocytoma cells. J Cell Physiol 129, 71-76.

Guilding, C., McNair, K., Stone, T.W., and Morris, B.J. (2007). Restored plasticity in a mouse model of neurofibromatosis type 1 via inhibition of hyperactive ERK and CREB. Eur J Neurosci 25, 99-105.

Haberman, R.P., Lee, H.J., Colantuoni, C., Koh, M.T., and Gallagher, M. (2008). Rapid encoding of new information alters the profile of plasticity-related mRNA transcripts in the hippocampal CA3 region - Supplementary material.

Hancock, J.F. (2003). Ras proteins: different signals from different locations. Nat Rev Mol Cell Biol 4, 373-384.

Harvey, C.D., Yasuda, R., Zhong, H., and Svoboda, K. (2008). The spread of Ras activity triggered by activation of a single dendritic spine. Science 321, 136-140.

Harvey, J.J. (1964). An Unidentified Virus Which Causes the Rapid Production of Tumours in Mice. Nature 204, 1104-1105.

Hayashi, Y., Shi, S.H., Esteban, J.A., Piccini, A., Poncer, J.C., and Malinow, R. (2000). Driving AMPA receptors into synapses by LTP and CaMKII: requirement for GluR1 and PDZ domain interaction. Science 287, 2262-2267. 
Helmreich, E.J. (2004). Structural flexibility of small GTPases. Can it explain their functional versatility? Biol Chem 385, 1121-1136.

Herzog, E., Takamori, S., Jahn, R., Brose, N., and Wojcik, S.M. (2006). Synaptic and vesicular co-localization of the glutamate transporters VGLUT1 and VGLUT2 in the mouse hippocampus. J Neurochem 99, 1011-1018.

Heumann, R., Goemans, C., Bartsch, D., Lingenhohl, K., Waldmeier, P.C., Hengerer, B., Allegrini, P.R., Schellander, K., Wagner, E.F., Arendt, T., et al. (2000). Transgenic activation of Ras in neurons promotes hypertrophy and protects from lesion-induced degeneration. J Cell Biol 151, 1537-1548.

Holzer, M., Rodel, L., Seeger, G., Gartner, U., Narz, F., Janke, C., Heumann, R., and Arendt, T. (2001). Activation of mitogen-activated protein kinase cascade and phosphorylation of cytoskeletal proteins after neurone-specific activation of p21ras. II. Cytoskeletal proteins and dendritic morphology. Neuroscience 105, 1041-1054.

Homayouni, R., Magdaleno, S., Keshvara, L., Rice, D.S., and Curran, T. (2003). Interaction of Disabled-1 and the GTPase activating protein Dab2IP in mouse brain. Brain Res Mol Brain Res 115, 121-129.

Honoré, T., Lauridsen, J., and Krogsgaard-Larsen, P. (1982). The binding of [3H]AMPA, a structural analogue of glutamic acid, to rat brain membranes. J Neurochem 38, 173-178.

Hoogenraad, C.C., Popa, I., Futai, K., Sanchez-Martinez, E., Wulf, P.S., van Vlijmen, T., Dortland, B.R., Oorschot, V., Govers, R., Monti, M., et al. (2010). Neuron specific Rab4 effector GRASP-1 coordinates membrane specialization and maturation of recycling endosomes. PLoS Biol 8, e1000283.

Horton, A.C., Racz, B., Monson, E.E., Lin, A.L., Weinberg, R.J., and Ehlers, M.D. (2005). Polarized secretory trafficking directs cargo for asymmetric dendrite growth and morphogenesis. Neuron 48, 757-771.

Hoshi, M., Nishida, E., and Sakai, H. (1988). Activation of a Ca2+-inhibitable protein kinase that phosphorylates microtubule-associated protein 2 in vitro by growth factors, phorbol esters, and serum in quiescent cultured human fibroblasts. J Biol Chem 263, 5396-5401.

Huang, J., Furuya, A., and Furuichi, T. (2007). Very-KIND, a KIND domain containing RasGEF, controls dendrite growth by linking Ras small GTPases and MAP2. J Cell Biol 179, 539-552.

Hunt, C.A., Schenker, L.J., and Kennedy, M.B. (1996). PSD-95 is associated with the postsynaptic density and not with the presynaptic membrane at forebrain synapses. $J$ Neurosci 16, 1380-1388. 
Husi, H., Ward, M.A., Choudhary, J.S., Blackstock, W.P., and Grant, S.G. (2000). Proteomic analysis of NMDA receptor-adhesion protein signaling complexes. Nat Neurosci 3, 661-669.

Huttner, W.B., Schiebler, W., Greengard, P., and De Camilli, P. (1983). Synapsin I (protein I), a nerve terminal-specific phosphoprotein. III. Its association with synaptic vesicles studied in a highly purified synaptic vesicle preparation. J Cell Biol 96, 13741388.

Imamura, Y., Matsumoto, N., Kondo, S., Kitayama, H., and Noda, M. (2003). Possible involvement of Rap1 and Ras in glutamatergic synaptic transmission. Neuroreport 14, 1203-1207.

Irie, M., Hata, Y., Takeuchi, M., Ichtchenko, K., Toyoda, A., Hirao, K., Takai, Y., Rosahl, T.W., and Südhof, T.C. (1997). Binding of neuroligins to PSD-95. Science 277, $1511-1515$.

Jaworski, J., Spangler, S., Seeburg, D.P., Hoogenraad, C.C., and Sheng, M. (2005). Control of dendritic arborization by the phosphoinositide-3'-kinase-Akt-mammalian target of rapamycin pathway. J Neurosci 25, 11300-11312.

Jin, T.G., Satoh, T., Liao, Y., Song, C., Gao, X., Kariya , K., Hu, C.D., and Kataoka, T. (2001). Role of the CDC25 homology domain of phospholipase Cepsilon in amplification of Rap1-dependent signaling. J Biol Chem 276, 30301-30307.

Karnoub, A.E., and Weinberg, R.A. (2008). Ras oncogenes: split personalities. Nat Rev Mol Cell Biol 9, 517-531.

Kawasaki, H., Springett, G.M., Toki, S., Canales, J.J., Harlan, P., Blumenstiel, J.P., Chen, E.J., Bany, I.A., Mochizuki, N., Ashbacher, A., et al. (1998). A Rap guanine nucleotide exchange factor enriched highly in the basal ganglia. Proc Natl Acad Sci U S A 95, 13278-13283.

Kennedy, M.J., and Ehlers, M.D. (2006). Organelles and Trafficking Machinery for Postsynaptic Plasticity. Annu Rev Neurosci.

Kielland, A., Bochorishvili, G., Corson, J., Zhang, L., Rosin, D.L., Heggelund, P., and Zhu, J.J. (2009). Activity patterns govern synapse-specific AMPA receptor trafficking between deliverable and synaptic pools. Neuron 62, 84-101.

Kim, J.H., Lee, H.K., Takamiya, K., and Huganir, R.L. (2003). The role of synaptic GTPase-activating protein in neuronal development and synaptic plasticity. J Neurosci 23, 1119-1124.

Kim, K., Yang, J., Zhong, X.P., Kim, M.H., Kim, Y.S., Lee, H.W., Han, S., Choi, J., Han, K., Seo, J., et al. (2009). Synaptic removal of diacylglycerol by DGKzeta and PSD-95 regulates dendritic spine maintenance. EMBO J 28, 1170-1179. 
Klausberger, T., and Somogyi, P. (2008). Neuronal diversity and temporal dynamics: the unity of hippocampal circuit operations. Science $321,53-57$.

Ko, B., Joshi, L.M., Cooke, L.L., Vazquez, N., Musch, M.W., Hebert, S.C., Gamba, G., and Hoover, R.S. (2007). Phorbol ester stimulation of RasGRP1 regulates the sodium-chloride cotransporter by a PKC-independent pathway. Proc Natl Acad Sci USA 104, 20120-20125.

Kuiperij, H.B., de Rooij, J., Rehmann, H., van Triest, M., Wittinghofer, A., Bos, J.L., and Zwartkruis, F.J. (2003). Characterisation of PDZ-GEFs, a family of guanine nucleotide exchange factors specific for Rap1 and Rap2. Biochim Biophys Acta 1593, 141-149.

Kumar, V., Zhang, M.X., Swank, M.W., Kunz, J., and Wu, G.Y. (2005). Regulation of dendritic morphogenesis by Ras-PI3K-Akt-mTOR and Ras-MAPK signaling pathways. J Neurosci 25, 11288-11299.

Kushner, S.A., Elgersma, Y., Murphy, G.G., Jaarsma, D., van Woerden, G.M., Hojjati, M.R., Cui, Y., LeBoutillier, J.C., Marrone, D.F., Choi, E.S., et al. (2005). Modulation of presynaptic plasticity and learning by the $\mathrm{H}$-ras/extracellular signalregulated kinase/synapsin I signaling pathway. J Neurosci 25, 9721-9734.

Leevers, S.J., and Marshall, C.J. (1992). Activation of extracellular signal-regulated kinase, ERK2, by p21ras oncoprotein. EMBO J 11, 569-574.

Leon, J., Guerrero, I., and Pellicer, A. (1987). Differential expression of the ras gene family in mice. Molecular and Cellular Biology 7, 1535-1540.

Li, S., Tian, X., Hartley, D.M., and Feig, L.A. (2006). Distinct roles for Ras-guanine nucleotide-releasing factor 1 (Ras-GRF1) and Ras-GRF2 in the induction of longterm potentiation and long-term depression. J Neurosci 26, 1721-1729.

Lisman, J., and Raghavachari, S. (2006). A unified model of the presynaptic and postsynaptic changes during LTP at CA1 synapses. Sci STKE 2006, re11.

López De Jesús, M., Stope, M.B., Oude Weernink, P.A., Mahlke, Y., Börgermann, C., Ananaba, V.N., Rimmbach, C., Rosskopf, D., Michel, M.C., Jakobs, K.H., et al. (2006). Cyclic AMP-dependent and Epac-mediated activation of R-Ras by G proteincoupled receptors leads to phospholipase D stimulation. J Biol Chem 281, 2183721847.

Lopez, I., Mak, E.C., Ding, J., Hamm, H.E., and Lomasney, J.W. (2001). A novel bifunctional phospholipase $\mathrm{c}$ that is regulated by Galpha 12 and stimulates the Ras/mitogen-activated protein kinase pathway. J Biol Chem 276, 2758-2765.

Lorenzo, P.S., Kung, J.W., Bottorff, D.A., Garfield, S.H., Stone, J.C., and Blumberg, P.M. (2001). Phorbol esters modulate the Ras exchange factor RasGRP3. Cancer Res 61, 943-949. 
Manabe, T., Aiba, A., Yamada, A., Ichise, T., Sakagami, H., Kondo, H., and Katsuki, M. (2000). Regulation of long-term potentiation by $\mathrm{H}$-Ras through NMDA receptor phosphorylation. J Neurosci 20, 2504-2511.

Mandai, K., Nakanishi, H., Satoh, A., Obaishi, H., Wada, M., Nishioka, H., Itoh, M., Mizoguchi, A., Aoki, T., Fujimoto, T., et al. (1997). Afadin: A novel actin filamentbinding protein with one PDZ domain localized at cadherin-based cell-to-cell adherens junction. J Cell Biol 139, 517-528.

Martegani, E., Vanoni, M., Zippel, R., Coccetti, P., Brambilla, R., Ferrari, C., Sturani, E., and Alberghina, L. (1992). Cloning by functional complementation of a mouse cDNA encoding a homologue of CDC25, a Saccharomyces cerevisiae RAS activator. EMBO J 11, 2151-2157.

Matus, A. (1994). Stiff microtubules and neuronal morphology. Trends Neurosci 17, 19-22.

Mátyás, F., Freund, T.F., and Gulyás, A.I. (2004). Immunocytochemically defined interneuron populations in the hippocampus of mouse strains used in transgenic technology. Hippocampus 14, 460-481.

McAllister, A.K. (2000). Cellular and molecular mechanisms of dendrite growth. Cereb Cortex 10, 963-973.

McAllister, A.K. (2007). Dynamic aspects of CNS synapse formation. Annu Rev Neurosci 30, 425-450.

Mees, A., Rock, R., Ciccarelli, F.D., Leberfinger, C.B., Borawski, J.M., Bork, P., Wiese, S., Gessler, M., and Kerkhoff, E. (2005). Very-KIND is a novel nervous system specific guanine nucleotide exchange factor for Ras GTPases. Gene Expr Patterns 6, 79-85.

Mizoguchi, A., Ueda, T., Ikeda, K., Shiku, H., and Mizoguti, H. (1989). Localization and subcellular distribution of cellular ras gene products in rat brain. Brain Res Mol Brain Res.

Moon, I.S., Sakagami, H., Nakayama, J., and Suzuki, T. (2008). Differential distribution of synGAP alpha1 and synGAP beta isoforms in rat neurons. Brain Res $1241,62-75$.

Müller, J., Cacace, A.M., Lyons, W.E., McGill, C.B., and Morrison, D.K. (2000). Identification of B-KSR1, a novel brain-specific isoform of KSR1 that functions in neuronal signaling. Molecular and Cellular Biology 20, 5529-5539.

Nakamura, T., Aoki, K., and Matsuda, M. (2005). Monitoring spatio-temporal regulation of Ras and Rho GTPase with GFP-based FRET probes. Methods 37, 146153. 
Newpher, T.M., and Ehlers, M.D. (2008). Glutamate receptor dynamics in dendritic microdomains. Neuron 58, 472-497.

Ng, E.L., and Tang, B.L. (2008). Rab GTPases and their roles in brain neurons and glia. Brain research reviews 58, 236-246.

Noda, M., Ko, M., Ogura, A., Liu, D.G., Amano, T., Takano, T., and Ikawa, Y. (1985). Sarcoma viruses carrying ras oncogenes induce differentiation-associated properties in a neuronal cell line. Nature 318, 73-75.

Nordlund, M., Gu, X., Shipley, M.T., and Ratner, N. (1993). Neurofibromin is enriched in the endoplasmic reticulum of CNS neurons. J Neurosci 13, 1588-1600.

Nuñez Rodriguez, N., Lee, I.N., Banno, A., Qiao, H.F., Qiao, R.F., Yao, Z., Hoang, T., Kimmelman, A.C., and Chan, A.M. (2006). Characterization of R-ras $3 / m-$ ras null mice reveals a potential role in trophic factor signaling. Molecular and Cellular Biology 26, 7145-7154.

Oh, J.S., Manzerra, P., and Kennedy, M.B. (2004). Regulation of the neuron-specific Ras GTPase-activating protein, synGAP, by $\mathrm{Ca} 2+/$ calmodulin-dependent protein kinase II. J Biol Chem 279, 17980-17988.

Ohba, Y., Mochizuki, N., Yamashita, S., Chan, A.M., Schrader, J.W., Hattori, S., Nagashima, K., and Matsuda, M. (2000). Regulatory proteins of R-Ras, TC21/RRas2, and M-Ras/R-Ras3. J Biol Chem 275, 20020-20026.

Oinuma, I., Katoh, H., and Negishi, M. (2007). R-Ras controls axon specification upstream of glycogen synthase kinase-3beta through integrin-linked kinase. J Biol Chem 282, 303-318.

Omerovic, J., and Prior, I.A. (2009). Compartmentalized signalling: Ras proteins and signalling nanoclusters. FEBS J 276, 1817-1825.

OTSU, N. (1979). A threshold selection method from graylevel histograms. IEEE Trans Syst, Madn \& Cybern 9, 62-66.

Peng, S.L. (2009). Altered T and B lymphocyte signaling pathways in lupus. Autoimmun Rev 8, 179-183.

Pham, N., Cheglakov, I., Koch, C.A., de Hoog, C.L., Moran, M.F., and Rotin, D. (2000). The guanine nucleotide exchange factor CNrasGEF activates ras in response to cAMP and cGMP. Curr Biol 10, 555-558.

Pierret, P., Dunn, R.J., Djordjevic, B., Stone, J.C., and Richardson, P.M. (2000). Distribution of ras guanyl releasing protein (RasGRP) mRNA in the adult rat central nervous system. J Neurocytol 29, 485-497. 
Pierret, P., Vallée, A., Mechawar, N., Dower, N.A., Stone, J.C., Richardson, P.M., and Dunn, R.J. (2001). Cellular and subcellular localization of Ras guanyl nucleotidereleasing protein in the rat hippocampus. Neuroscience 108, 381-390.

Pokorny, J., and Trojan, S. (1986). The development of hippocampal structure and how it is influenced by hypoxia. Acta Univ Carol Med Monogr 113, 1-79.

Porter, K., Komiyama, N.H., Vitalis, T., Kind, P.C., and Grant, S.G. (2005). Differential expression of two NMDA receptor interacting proteins, PSD-95 and SynGAP during mouse development. Eur J Neurosci 21, 351-362.

Priatel, J.J., Chen, X., Dhanji, S., Abraham, N., and Teh, H.S. (2006). RasGRP1 transmits prodifferentiation TCR signaling that is crucial for CD4 T cell development. J Immunol 177, 1470-1480.

Purkyně, J.E. (1838). Bericht über die Versammlung deutcher Naturforscher und Ärzte im September, 1837. Reviewd in 'Minds behind the Brain - A history of the Pioneers and Their Discoveries' by Stanley Finger, PhD, Oxford University Press 0 , 200-202.

Qin, Y., Zhu, Y., Baumgart, J.P., Stornetta, R.L., Seidenman, K., Mack, V., van Aelst, L., and Zhu, J.J. (2005). State-dependent Ras signaling and AMPA receptor trafficking. Genes Dev 19, 2000-2015.

Ramón y Cajal, S. (1888). Estructura de los centros nerviosos de las aves. Rev Trim Histol Norm Pat, reviewd in 'Minds behind the Brain - A history of the Pioneers and Their Discoveries' by Stanley Finger, PhD, Oxford University Press 0, 200-211.

Ray, L.B., and Sturgill, T.W. (1987). Rapid stimulation by insulin of a serine/threonine kinase in 3T3-L1 adipocytes that phosphorylates microtubule-associated protein 2 in vitro. Proc Natl Acad Sci USA 84, 1502-1506.

Rebhun, J.F., Castro, A.F., and Quilliam, L.A. (2000). Identification of guanine nucleotide exchange factors (GEFs) for the Rap1 GTPase. Regulation of MR-GEF by M-Ras-GTP interaction. J Biol Chem 275, 34901-34908.

Reuther, G.W., Lambert, Q.T., Rebhun, J.F., Caligiuri, M.A., Quilliam, L.A., and Der, C.J. (2002). RasGRP4 is a novel Ras activator isolated from acute myeloid leukemia. J Biol Chem 277, 30508-30514.

Ridler, T., and Calvard, S. (1978). Picture Thresholding Using an Iterative Selection Method. Systems, Man and Cybernetics, IEEE Transactions on 8, 630-632.

Rodriguez-Viciana, P., Warne, P.H., Dhand, R., Vanhaesebroeck, B., Gout, I., Fry, M.J., Waterfield, M.D., and Downward, J. (1994). Phosphatidylinositol-3-OH kinase as a direct target of Ras. Nature 370, 527-532. 
Roscioni, S.S., Elzinga, C.R., and Schmidt, M. (2008). Epac: effectors and biological functions. Naunyn Schmiedebergs Arch Pharmacol 377, 345-357.

Rosen, L.B., Ginty, D.D., Weber, M.J., and Greenberg, M.E. (1994). Membrane depolarization and calcium influx stimulate MEK and MAP kinase via activation of Ras. Neuron 12, 1207-1221.

Rosenmund, C., Stern-Bach, Y., and Stevens, C.F. (1998). The tetrameric structure of a glutamate receptor channel. Science 280, 1596-1599.

Rumbaugh, G., Adams, J.P., Kim, J.H., and Huganir, R.L. (2006). SynGAP regulates synaptic strength and mitogen-activated protein kinases in cultured neurons. Proc Natl Acad Sci USA 103, 4344-4351.

Saito, Y., Oinuma, I., Fujimoto, S., and Negishi, M. (2009). Plexin-B1 is a GTPase activating protein for M-Ras, remodelling dendrite morphology. EMBO Rep 10, 614621.

Sánchez, C., Díaz-Nido, J., and Avila, J. (2000). Phosphorylation of microtubuleassociated protein 2 (MAP2) and its relevance for the regulation of the neuronal cytoskeleton function. Prog Neurobiol 61, 133-168.

Schlüter, O.M., Xu, W., and Malenka, R. (2006). Alternative N-terminal domains of PSD-95 and SAP97 govern activity-dependent regulation of synaptic AMPA receptor function. Neuron 51, 99-111.

Schnell, E., Sizemore, M., Karimzadegan, S., Chen, L., Bredt, D.S., and Nicoll, R.A. (2002). Direct interactions between PSD-95 and stargazin control synaptic AMPA receptor number. Proc Natl Acad Sci USA 99, 13902-13907.

Schnutgen, F., Doerflinger, N., Calleja, C., Wendling, O., Chambon, P., and Ghyselinck, N.B. (2003). A directional strategy for monitoring Cre-mediated recombination at the cellular level in the mouse. Nat Biotechnol 21, 562-565.

Schoch, S., Castillo, P.E., Jo, T., Mukherjee, K., Geppert, M., Wang, Y., Schmitz, F., Malenka, R.C., and Südhof, T.C. (2002). RIM1alpha forms a protein scaffold for regulating neurotransmitter release at the active zone. Nature 415, 321-326.

Seeger, G., Gärtner, U., and Arendt, T. (2005). Transgenic activation of Ras in neurons increases synapse formation in mouse neocortex. J Neural Transm 112, 751-761.

Seeger, G., Gartner, U., Holzer, M., and Arendt, T. (2003). Constitutive expression of p21 H-Ras(Val12) in neurons induces increased axonal size and dendritic microtubule density in vivo. J Neurosci Res 74, 868-874.

Seeger, G., Yan, L., Gärtner, U., and Huemmeke, M. (2004). Activation of Ras in neurons modifies synaptic vesicle docking and release. Neuroreport. 
Sheng, M., Cummings, J., Roldan, L.A., Jan, Y.N., and Jan, L.Y. (1994). Changing subunit composition of heteromeric NMDA receptors during development of rat cortex. Nature 368, 144-147.

Shi, S., Hayashi, Y., Esteban, J.A., and Malinow, R. (2001). Subunit-specific rules governing AMPA receptor trafficking to synapses in hippocampal pyramidal neurons. Cell 105, 331-343.

Sholl, D.A. (1953). Dendritic organization in the neurons of the visual and motor cortices of the cat. J Anat 87, 387-406.

Shou, C., Farnsworth, C.L., Neel, B.G., and Feig, L.A. (1992). Molecular cloning of cDNAs encoding a guanine-nucleotide-releasing factor for Ras p21. Nature 358, 351354.

Signore, A.P., O'Rourke, F., Lu, X., Feinstein, M.B., and Yeh, H.H. (1999). Immunohistochemical localization of the INsP4 receptor GTPase-activating protein GAP1IP4BP in the rat brain. J Neurosci Res 55, 321-328.

Siksou, L., Rostaing, P., Lechaire, J.P., Boudier, T., Ohtsuka, T., Fejtová, A., Kao, H.T., Greengard, P., Gundelfinger, E.D., Triller, A., et al. (2007). Three-dimensional architecture of presynaptic terminal cytomatrix. J Neurosci 27, 6868-6877.

Siksou, L., Triller, A., and Marty, S. (2009). An emerging view of presynaptic structure from electron microscopic studies. J Neurochem 108, 1336-1342.

Song, I., and Huganir, R.L. (2002). Regulation of AMPA receptors during synaptic plasticity. Trends Neurosci 25, 578-588.

Spruston, N. (2008). Pyramidal neurons: dendritic structure and synaptic integration. Nat Rev Neurosci 9, 206-221.

Stein, V., House, D.R., Bredt, D.S., and Nicoll, R.A. (2003). Postsynaptic density-95 mimics and occludes hippocampal long-term potentiation and enhances long-term depression. J Neurosci 23, 5503-5506.

Stenmark, H. (2009). Rab GTPases as coordinators of vesicle traffic. Nat Rev Mol Cell Biol 10, 513-525.

Sternberg, S.R. (1983). Biomedical Image Processing. Computer 16, 22-34.

Stryer, L.M., Tymoczko, J.L., and Berg, J.M. (2002). Biochemistry, Fifth Edition. W H Freeman and Company -

Sturani, E., Abbondio, A., Branduardi, P., Ferrari, C., Zippel, R., Martegani, E., Vanoni, M., and Denis-Donini, S. (1997). The Ras Guanine nucleotide Exchange Factor CDC25Mm is present at the synaptic junction. Exp Cell Res 235, 117-123. 
Suzuki, T., Mitake, S., and Murata, S. (1999). Presence of up-stream and downstream components of a mitogen-activated protein kinase pathway in the PSD of the rat forebrain. Brain Res 840, 36-44.

Takai, Y., Sasaki, T., and Matozaki, T. (2001). Small GTP-binding proteins. Physiol Rev 81, 153-208.

Tall, G.G., Barbieri, M.A., Stahl, P.D., and Horazdovsky, B.F. (2001). Ras-activated endocytosis is mediated by the Rab5 guanine nucleotide exchange activity of RIN1. Dev Cell 1, 73-82.

Tang, B.L. (2008). Emerging aspects of membrane traffic in neuronal dendrite growth. Biochim Biophys Acta 1783, 169-176.

Tazmini, G., Beaulieu, N., Woo, A., Zahedi, B., Goulding, R.E., and Kay, R.J. (2009). Membrane localization of RasGRP1 is controlled by an EF-hand, and by the GEF domain. Biochim Biophys Acta 1793, 447-461.

Tian, X., Gotoh, T., Tsuji, K., Lo, E.H., Huang, S., and Feig, L.A. (2004). Developmentally regulated role for Ras-GRFs in coupling NMDA glutamate receptors to Ras, Erk and CREB. EMBO J 23, 1567-1575.

Toki, S., Kawasaki, H., Tashiro, N., Housman, D.E., and Graybiel, A.M. (2001). Guanine nucleotide exchange factors CalDAG-GEFI and CalDAG-GEFII are colocalized in striatal projection neurons. J Comp Neurol 437, 398-407.

Tonini, R., Franceschetti, S., Parolaro, D., Sala, M., Mancinelli, E., Tininini, S., Brusetti, R., Sancini, G., Brambilla, R., Martegani, E., et al. (2001). Involvement of CDC25Mm/Ras-GRF1-dependent signaling in the control of neuronal excitability. Mol Cell Neurosci 18, 691-701.

Vazquez, L.E., Chen, H.J., Sokolova, I., Knuesel, I., and Kennedy, M.B. (2004). SynGAP regulates spine formation. J Neurosci 24, 8862-8872.

Vincent, L., and Soille, P. (1991). Watersheds in Digital Spaces: An Efficient Algorithm Based on Immersion Simulations. IEEE Transactions on Pattern Analysis and Machine Intelligence 13, 583-598.

Vojtek, A.B., Hollenberg, S.M., and Cooper, J.A. (1993). Mammalian Ras interacts directly with the serine/threonine kinase Raf. Cell 74, 205-214.

Wang, D.Z., Hammond, V.E., Abud, H.E., Bertoncello, I., McAvoy, J.W., and Bowtell, D.D. (1997). Mutation in Sos1 dominantly enhances a weak allele of the EGFR, demonstrating a requirement for Sos1 in EGFR signaling and development. Genes Dev 11, 309-320.

Warne, P.H., Viciana, P.R., and Downward, J. (1993). Direct interaction of Ras and the amino-terminal region of Raf-1 in vitro. Nature 364, 352-355. 
Wayman, G.A., Impey, S., Marks, D., Saneyoshi, T., Grant, W.F., Derkach, V., and Soderling, T.R. (2006). Activity-dependent dendritic arborization mediated by CaMkinase I activation and enhanced CREB-dependent transcription of Wnt-2. Neuron 50, 897-909.

Wei, P., Satoh, T., Edamatsu, H., Aiba, A., Setsu, T., Terashima, T., Kitazawa, S., Nakao, K., Yoshikawa, Y., Tamada, M., et al. (2007). Defective vascular morphogenesis and mid-gestation embryonic death in mice lacking RA-GEF-1. Biochem Biophys Res Commun 363, 106-112.

Wei, W., Schreiber, S.S., Baudry, M., Tocco, G., and Broek, D. (1993). Localization of the cellular expression pattern of cdc25NEF and ras in the juvenile rat brain. Brain Res Mol Brain Res 19, 339-344.

Willingham, M.C., Pastan, I., Shih, T.Y., and Scolnick, E.M. (1980). Localization of the src gene product of the Harvey strain of MSV to plasma membrane of transformed cells by electron microscopic immunocytochemistry. Cell 19, 1005-1014.

Wong, R.O., and Ghosh, A. (2002). Activity-dependent regulation of dendritic growth and patterning. Nat Rev Neurosci 3, 803-812.

Wood, K.W., Sarnecki, C., Roberts, T.M., and Blenis, J. (1992). ras mediates nerve growth factor receptor modulation of three signal-transducing protein kinases: MAP kinase, Raf-1, and RSK. Cell 68, 1041-1050.

Wu, G.Y., Deisseroth, K., and Tsien, R.W. (2001). Spaced stimuli stabilize MAPK pathway activation and its effects on dendritic morphology. Nat Neurosci 4, 151-158.

Yamashita, S., Mochizuki, N., Ohba, Y., Tobiume, M., Okada, Y., Sawa, H., Nagashima, K., and Matsuda, M. (2000). CalDAG-GEFIII activation of Ras, R-ras, and Rap1. J Biol Chem 275, 25488-25493.

Yang, H., and Mattingly, R.R. (2006). The Ras-GRF1 exchange factor coordinates activation of $\mathrm{H}$-Ras and Rac1 to control neuronal morphology. Mol Biol Cell 17, 21772189.

Yang, Y., Li, L., Wong, G.W., Krilis, S.A., Madhusudhan, M.S., Sali, A., and Stevens, R.L. (2002). RasGRP4, a new mast cell-restricted Ras guanine nucleotide-releasing protein with calcium- and diacylglycerol-binding motifs. Identification of defective variants of this signaling protein in asthma, mastocytosis, and mast cell leukemia patients and demonstration of the importance of RasGRP4 in mast cell development and function. J Biol Chem 277, 25756-25774.

Yarwood, S., Bouyoucef-Cherchalli, D., Cullen, P.J., and Kupzig, S. (2006). The GAP1 family of GTPase-activating proteins: spatial and temporal regulators of small GTPase signalling. Biochem Soc Trans 34, 846-850. 
Yasuda, R., Harvey, C.D., Zhong, H., Sobczyk, A., van Aelst, L., and Svoboda, K. (2006). Supersensitive Ras activation in dendrites and spines revealed by twophoton fluorescence lifetime imaging. Nat Neurosci 9, 283-291.

Ye, B., Liao, D., Zhang, X., Zhang, P., Dong, H., and Huganir, R.L. (2000). GRASP1: a neuronal RasGEF associated with the AMPA receptor/GRIP complex. Neuron $26,603-617$.

Yoshihara, Y., De Roo, M., and Muller, D. (2009). Dendritic spine formation and stabilization. Current Opinion in Neurobiology 19, 146-153.

Yoshimura, T., Arimura, N., Kawano, Y., Kawabata, S., Wang, S., and Kaibuchi, K. (2006). Ras regulates neuronal polarity via the PI3-kinase/Akt/GSK-3beta/CRMP-2 pathway. Biochem Biophys Res Commun 340, 62-68.

Zhang, X.F., Settleman, J., Kyriakis, J.M., Takeuchi-Suzuki, E., Elledge, S.J., Marshall, M.S., Bruder, J.T., Rapp, U.R., and Avruch, J. (1993). Normal and oncogenic p21ras proteins bind to the amino-terminal regulatory domain of c-Raf-1. Nature 364, 308-313.

Zhu, J.J., Esteban, J.A., Hayashi, Y., and Malinow, R. (2000). Postnatal synaptic potentiation: delivery of GluR4-containing AMPA receptors by spontaneous activity. Nat Neurosci 3, 1098-1106.

Zhu, J.J., Qin, Y., Zhao, M., van Aelst, L., and Malinow, R. (2002). Ras and Rap control AMPA receptor trafficking during synaptic plasticity. Cell 110, 443-455.

Zhu, Y., and Parada, L.F. (2001). Neurofibromin, a tumor suppressor in the nervous system. Exp Cell Res 264, 19-28.

Zippel, R., Gnesutta, N., Matus-Leibovitch, N., Mancinelli, E., Saya, D., Vogel, Z., Sturani, E., Renata, Z., Nerina, G., Noa, M.L., et al. (1997). Ras-GRF, the activator of Ras, is expressed preferentially in mature neurons of the central nervous system. Brain Res Mol Brain Res 48, 140-144.

Zucker, R.S., and Regehr, W.G. (2002). Short-term synaptic plasticity. Annu Rev Physiol 64, 355-405. 


\section{Appendix}

\section{VIII.1. Strategy for the Knock-out of Rasgrp1 in Mice}

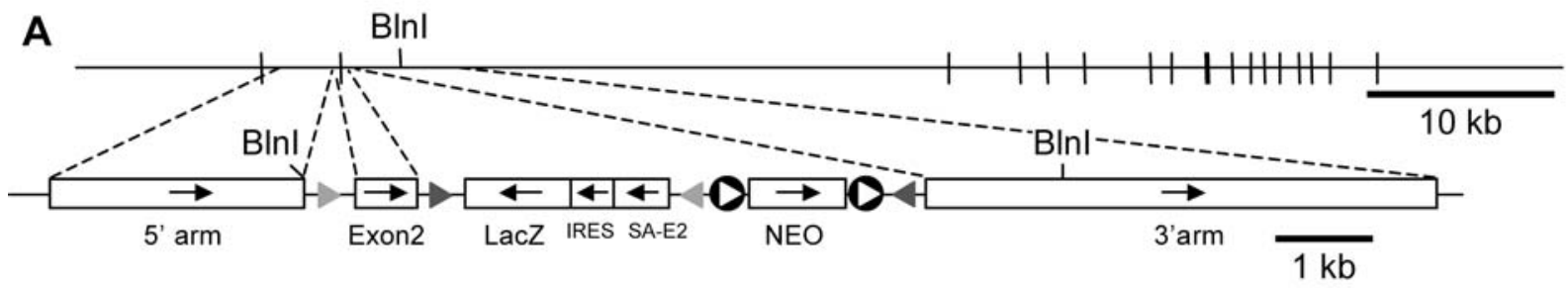

B

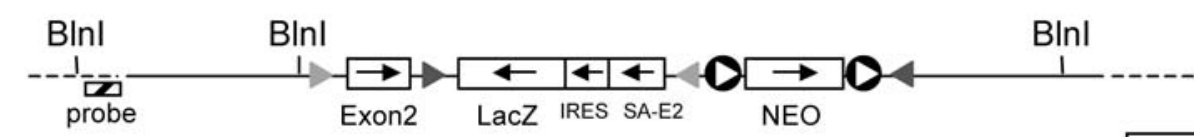

C



D

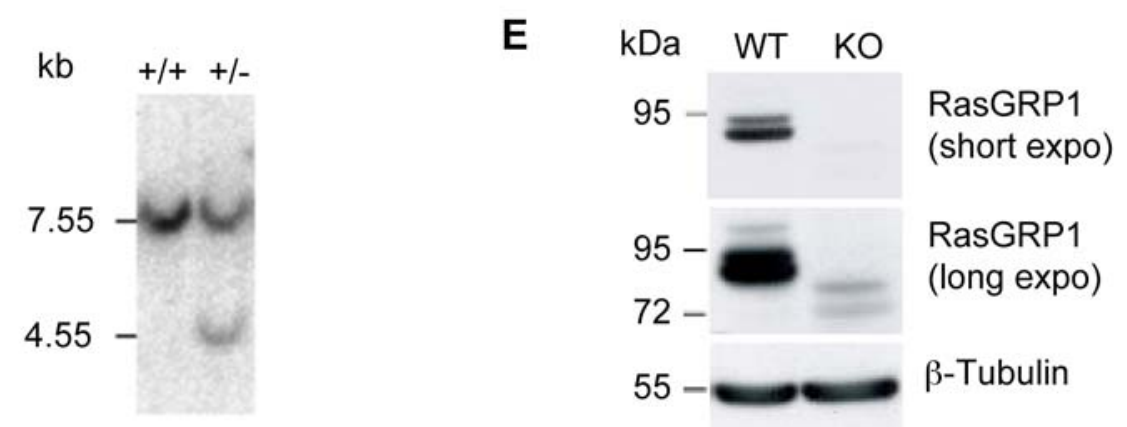

Figure VIII.1: Organization of the RasGRP1 gene and construction of the targeting vector in pFlexHR (Schnutgen et al., 2003)

(A) Organization of the wild-type (WT) gene. (B, C) The resulting genomic reorganizations of the RasGRP1 gene after homologous recombination and after Cre-recombination are shown below. (C) The resulting organization of the knock-out (KO) gene. (D) Wild-type ES cells and ES cells after homologous recombination were subjected to Southern Blot analysis after Blnl restriction. kb, kilo basepairs. (E) Western Blot analysis of RasGRP1 protein expression in wild-type and mutant animals. Whole brain homogenates were subjected to SDS-PAGE and Western Blot analysis with specific antibodies against RasGRP1 and $\beta$-Tubulin. kDa, kilo Dalton. Note that a weak signal is visible after long exposure, indicating very low expression of a Rasgrp1 fragment of decreased size. 


\section{VIII.2. Acknowledgements}

First of all, I would like to express my sincere gratitude towards Dr. Andrea Betz and Prof. Dr. Nils Brose. Dr. Andrea Betz introduced me to Rasgrp1 as the subjects of my thesis, taught me how to design and to conduct experiments, and provided the Rasgrp1 KO mouse line without which this study would not have been possible. Our scientific communication, as well as her encouragement and support, did not stop even after she had moved to the other side of this planet.

It may happen, that a PhD student experiences disorientation when the direct supervision by a specific person ends. That never occurred to me. After Dr. Andrea Betz had left to New Zealand, the input and the guidance of Prof. Dr. Nils Brose never even let me know traces of that experience. I will always be grateful to his educative and critical thoughts, the motivation and the support that helped so much to fulfill my work. I thank the members of my thesis committee Prof. Dr. Ralf Heinrich and Dr. Silvio Rizzoli for critically contemplating my work and providing valuable input that had great influence on my project. I am also grateful to Prof. Brose to have secured the funding by the Max-Plack-Association for the period of my PhD. Most of all I am indebted to him to include me as a member of his department. Here at the Max-Planck-Institute of Experimental Medicine in Göttingen I found an extraordinary atmosphere that stimulated the exchange of thoughts and know-how. I had the privilege to work alongside many excellent colleagues and friends in the Department of Molecular Biology: Dr. Aleksandra Ivanovic, Dr. Kerstin Reim, Dr. Friederike Wolk, Dr. Ben Cooper, Dr. Hiroshi Kawabe, Noa Lipsthein, Dr. Andrea Burgalossi, Dr. Tina Hoon and Dr. Etienne Herzog. I am also very thankful to have had an excellent technical assistance by Astrid Zeuch, Thea Hellmann, Anja Galinski and Martin Dörre. I appreciatively thank Inga Schauenberg and Astrid Ohle for caring for the Rasgrp1 mouse line and for never letting me doubt that these mice had a very good time in the animal facility of the institute. I am especially fortunate to have had Christoph Biesemann as a collaborator and friend, with whom I not only shared the joy and the frustrations in the life of PhD students, but also the thoughts on the past, the present and the plans for the future. During my PhD period I am grateful to have had two exceptional rotation students, Dominik von Wallhoffen and Maren Steinmetz.

Furthermore, I am thankful to the directors of the Max-Planck-Institutes in Göttingen who always supported a thriving community of PhD students. This community not only provided the breeding ground for collaborations and innovative 
ideas, but also for friendships. I am grateful to have known Christian Humml, who influenced me and a many other $\mathrm{PhD}$ students with his dedication for this community. I appreciate Julia Mathejczyk, Dr. Florian Hauer, Dr. Sven Wichert, Aiman Saab, Dr. Tobias Fischer and Alexander Pieper as my friends and collaborators.

I am grateful to my external collaborators Prof. Dr. Dietmar Schmitz and Dr. Jörg Breustedt to have given me the opportunity to perform the electrophysiological experiments described in the present study in their laboratory at the Charité University Hospital. I was very fortunate not only to have encountered excellent scientists who infected me with their passion for electrophysiology, but also to have found new friends that made my two long visits in Berlin unforgettable experiences. These people further include Dr. Benjamin Rost, Dr. Prateep Beed, Dr. Jochen Winter, Dr. Alekander Zivkovic and Michael Kintscher. I am also thankful to the technical assistance that was provided by Anke Schönherr and Susanne Walden. The Max-Planck fellows Dr. Martje Tönjes, Linda Hallen, Dr. Axel Rasche, Dr. Lukas Chavez, my landlords Dr. Wilhelm-Friedbert and Geesche Lohoff and my friends Dr. Holger Fricke and Nils Jansen completed the friendly and stimulating atmosphere in Berlin.

I am deeply indebted to my "BIFfly" friends and doctors to-be Stefan Friederichs, Markus Schneider, Jan Syring and Simon Schäfer. Although being friends for a long time already, this adventurous circle has become a precious source of intellectual recreation during the past four years. I also thank doctor to-be Jacob Schirmer and Johannes Bankwitz for interdisciplinary discussions and innovative exchange of thoughts during lunchtime.

Finally and most importantly, I am grateful to my family. I thank my dad Dieter Bungers, my brother Tobias Bungers, my uncle Hans G. Bungers and my aunt Barbara Bungers for having graciously and lovingly provided for me throughout. I thank my mom for having guarded over me all my life, and I thank Nina for simply being there. 


\section{VIII.3. Curriculum Vitae}

\section{Simon Rolf Bungers}

Born in Bonn on the $7^{\text {th }}$ of Dec., 1979

\begin{tabular}{|c|c|c|}
\hline 1990 & $\begin{array}{c}\text { May } \\
1999\end{array}$ & $\begin{array}{c}\text { Secondary school in Bonn (Kardinal-Frings-Gymnasium Bonn-Beuel) } \\
\text { and qualification for university admission with Abitur. }\end{array}$ \\
\hline $\begin{array}{l}\text { Jul. } \\
1999\end{array}$ & $\begin{array}{c}\text { Apr. } \\
-2000\end{array}$ & Military Service in the Navy of the Federal Republic of Germany. \\
\hline $\begin{array}{l}\text { May } \\
2000\end{array}$ & $\begin{array}{l}\text { Aug. } \\
2000\end{array}$ & $\begin{array}{l}\text { Internship at BASF AG, Ludwigshafen. In the main DNA facility I took } \\
\text { part in a project aimed at sequencing a specific plant genome. }\end{array}$ \\
\hline
\end{tabular}

Oct. - Oct. Study of Biology at the University of Würzburg, Germany, with majors in $2000-2005$

Mar. May

$2001-2001$

Dec. Feb.

$2001-2002$

Mar. Aug.

$2003-2003$

Sep. - Apr.

$2003-2004$

Jan. Mar.

$2005-2005$

Sep. - Jul.

2005 - 2006

Jul. 2006

since Oct. 2006
Neurobiology and Biochemistry.
First Internship at Memorec Stoffel GmbH (now Miltenyi Biotec), Cologne. In the group of Dr. Kai Hoffmann I performed basic bioinformatical work and database research.

Second internship at Memorec Stoffel GmbH. Continuation of the bioinformatical work, furthermore work in the course of a "drug target validation" project involving in-situ hybridization techniques

Visiting Student at the Rockefeller University, New York City, USA. In the laboratory of Prof. Dr. Markus Stoffel I made my first intense practical contact with molecular cloning.

Visiting Student at the Università degli Studi di Catania, Italy. In the laboratory of Prof. Dr. Vito di Pinto I learned basic biochemical techniques.

Laboratory rotation in the department of Clinical Neurobiology at the University Hospital Würzburg. Under supervision of Prof. Dr. Michael Sendtner and Prof. Dr. Stefan Wiese I acquired basic knowledge in immunohistochemical methods and Laser Scanning Microscopy.

Diploma thesis with the title "The Function of Rasgrp1 in Neurons" at the Max-Planck-Institute of Experimental Medicine in the laboratory of Prof.

Dr. Nils Brose. Under supervision of Dr. Andrea Betz I made my first experiments on Rasgrp1, involving overexpression of constructs in primary hippocampal cultures. I obtained data that indicated a possible function of Rasgrp1 in dendrite morphogenesis.

Graduation with honors and the degree "Diplom Biologe Univ." at the University of Würzburg.

PhD thesis with the title "The Role of the Ras Guanyl-Nucleotide Exchange Factor Rasgrp1 in Synaptic Transmission". 